\title{
The XMM-Newton extended survey of the Taurus molecular cloud $(\mathrm{XEST})^{\star, \star \star}$
}

\author{
M. Güdel ${ }^{1}$, K. R. Briggs ${ }^{1}$, K. Arzner ${ }^{1}$, M. Audard ${ }^{2, \star \star \star}$, J. Bouvier ${ }^{3}$, E. D. Feigelson ${ }^{4}$, E. Franciosini ${ }^{5}$, A. Glauser ${ }^{1}$, \\ N. Grosso ${ }^{3}$, G. Micela ${ }^{5}$, J.-L. Monin ${ }^{3}$, T. Montmerle ${ }^{3}$, D. L. Padgett ${ }^{6}$, F. Palla ${ }^{7}$, I. Pillitteri ${ }^{8}$, L. Rebull ${ }^{6}$, L. Scelsi ${ }^{8}$, \\ B. Silva ${ }^{9,10}$, S. L. Skinner ${ }^{11}$, B. Stelzer ${ }^{5}$, and A. Telleschi ${ }^{1}$
}

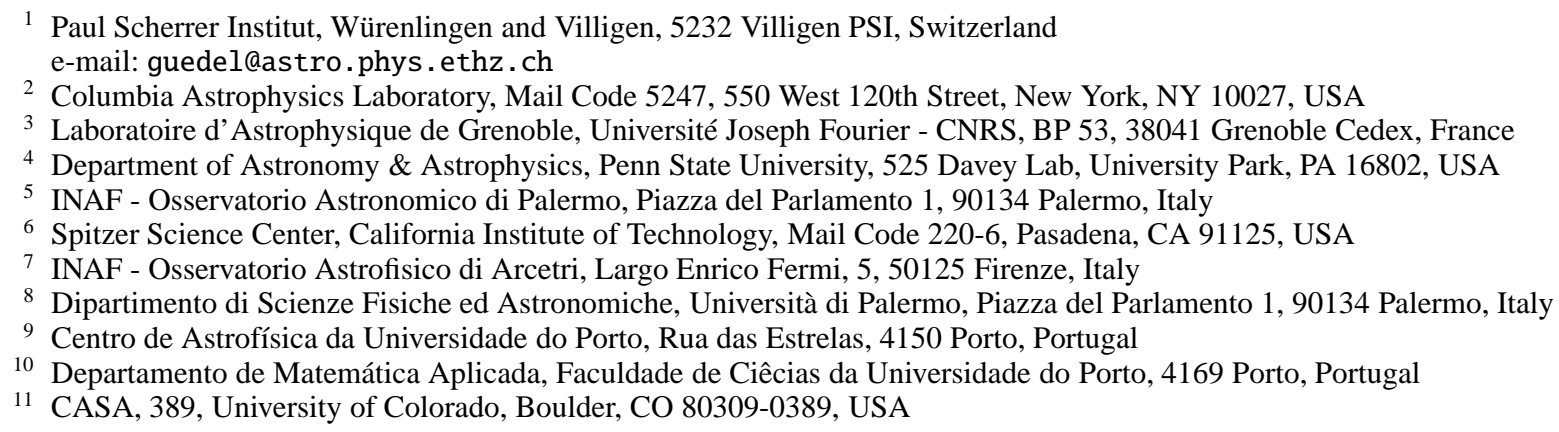

Received 31 May 2006 / Accepted 5 August 2006

ABSTRACT

\begin{abstract}
Context. The Taurus Molecular Cloud (TMC) is the nearest large star-forming region, prototypical for the distributed mode of lowmass star formation. Pre-main sequence stars are luminous X-ray sources, probably mostly owing to magnetic energy release.

Aims. The XMM-Newton Extended Survey of the Taurus Molecular Cloud (XEST) presented in this paper surveys the most populated $\approx 5$ square degrees of the TMC, using the XMM-Newton X-ray observatory to study the thermal structure, variability, and long-term evolution of hot plasma, to investigate the magnetic dynamo, and to search for new potential members of the association. Many targets are also studied in the optical, and high-resolution X-ray grating spectroscopy has been obtained for selected bright sources.

Methods. The X-ray spectra have been coherently analyzed with two different thermal models (2-component thermal model, and a continuous emission measure distribution model). We present overall correlations with fundamental stellar parameters that were derived from the previous literature. A few detections from Chandra observations have been added.

Results. The present overview paper introduces the project and provides the basic results from the X-ray analysis of all sources detected in the XEST survey. Comprehensive tables summarize the stellar properties of all targets surveyed. The survey goes deeper than previous X-ray surveys of Taurus by about an order of magnitude and for the first time systematically accesses very faint and strongly absorbed TMC objects. We find a detection rate of $85 \%$ and $98 \%$ for classical and weak-line T Tau stars (CTTS resp. WTTS), and identify about half of the surveyed protostars and brown dwarfs. Overall, 136 out of 169 surveyed stellar systems are detected. We describe an X-ray luminosity vs. mass correlation, discuss the distribution of X-ray-to-bolometric luminosity ratios, and show evidence for lower X-ray luminosities in CTTS compared to WTTS. Detailed analysis (e.g., variability, rotation-activity relations, influence of accretion on X-rays) will be discussed in a series of accompanying papers.
\end{abstract}

Key words. stars: formation - stars: pre-main sequence - X-rays: stars - gravitational waves

\section{Introduction}

Studies of star-forming regions have drawn a picture in which cool, molecular gas, contracting to protostars with surrounding accretion disks, co-exists with high-energy radiation. The

* Table 3, Full Tables 4-11 and Appendices A and B are only available in electronic form at http://www. aanda.org

$\star \star$ Full Table A.1 is only available in electronic form at the CDS via anonymous ftp to cdsarc.u-strasbg. fr (130.79.128.5) or via http://cdsweb.u-strasbg.fr/cgi-bin/qcat?J/A+A/468/353

$\star \star \star$ New address: Integral Science Data Centre, Ch. d'Ecogia 16, 1290 Versoix, and Geneva Observatory, University of Geneva, Ch. des Maillettes 51, 1290 Sauverny, Switzerland. latter is emitted by plasma in the stellar environment that is continuously heated to temperatures beyond one million degrees. This radiation is most prominently seen in the soft $(0.1-10 \mathrm{keV})$ $\mathrm{X}$-ray range and is conventionally attributed to the presence of magnetically trapped plasma in the outer stellar atmosphere. This plasma is reminiscent of, in the broadest sense, the solar coronal plasma. There is little doubt that both lower-energy (extreme ultraviolet) and higher-energy (hard X-ray and gamma - ray) radiation is amply present, but strong photoelectric absorption or, respectively, extremely low flux precludes direct detection. Further, non-thermal radio emission from relativistic electrons trapped in the magnetic fields gives unambiguous evidence for the presence of an accelerated population of electrons 
similar to populations seen in the solar and in stellar coronae during flares (Güdel 2002). Again, by implication from the solar analogy, we expect that high-energy, non-thermal particles (electrons and ions) play a crucial role also outside the confines of the magnetic stellar atmosphere.

But in contrast to the solar case, high-energy radiation from forming stars has a profound influence on its environment as dense accretion disks and the larger-scale molecular envelope are irradiated, heated, and ionized by high-energy photons and particles. There are numerous consequences of these processes. Ultraviolet and X-ray irradiation of molecular disks heats their upper layers (Alexander et al. 2004; Glassgold et al. 2004), making them accessible to magnetic fields, thus inducing, for example, the magnetorotational instability (Balbus \& Hawley 1991). The latter is thought to be a prime source for increased disk viscosity, which in turn is the main driver of the accretion process and thus the stellar formation process in the first place. Complex chemical networks are also put in operation by heating and ionizing the molecular environment (Glassgold et al. 2004), thus fundamentally altering composition, opacity, and cooling behavior of the material. Magnetic fields are thought to attach to the inner border of the lightly ionized accretion disk, thus transporting material to the stellar surface while at the same time applying torques to the star that may regulate its rotation period (Montmerle et al. 2000). If the stellar magnetic fields are generated in a solar-like dynamo in which rotation is a key parameter, then the long-term evolution of the magnetic field production and thus of the high-energy radiation is indeed directly controlled by the accretion process in the feedback loop sketched above. Further, magnetic fields and ionization of the disk may be relevant for the launch of bipolar jets and molecular outflows (Pudritz \& Norman 1986), a widely observed but poorly understood mechanism that seems to regulate the mass buildup of the forming star. Alternatively, these jets may originate from the magnetic fields spanning between the star and the disk (Shu et al. 1994), or from plasma clouds ejected in the process of magnetic reconnection of these same fields (Hayashi et al. 1996).

The origin of the X-ray emission is still not entirely clear. While the bulk of the X-rays from T Tau stars is thought to originate in a magnetically trapped corona consisting of complex magnetic loops anchored in photospheric magnetic regions like in the Sun, features unknown to the Sun may exist. Speculation has arisen that some very soft X-rays may be formed in accretion shocks near the stellar surface (e.g., Kastner et al. 2002). HerbigHaro objects far from the forming stars have been detected to be very soft X-ray emitters due to shock interactions with the interstellar medium (e.g., Pravdo et al. 2001; Bally et al. 2003; Güdel et al. 2005), but new spectral phenomenology suggests that appreciable X-ray emission may also be formed close to the stars, at the (spatially unresolved) base of the jets otherwise seen in radio and in the optical (Güdel et al. 2005). Whatever the analogy to the Sun, we also note that the level of the X-ray luminosity (between a few times $10^{28} \mathrm{erg} \mathrm{s}^{-1}$ and $10^{31} \mathrm{erg} \mathrm{s}^{-1}$ for low-mass pre-main sequence stars) and the measured plasma temperatures of up to $100 \mathrm{MK}$ are extreme and may imply heating mechanisms related to continuous flaring (Güdel et al. 2003; Wolk et al. 2005).

Studies of X-ray radiation in pre-main sequence stars are thus crucial for our further understanding of star formation, accretion processes, angular momentum transport, and mass outflow mechanisms in the earliest stages of a star's life. For recent reviews of X-rays from pre-main sequence stars, we refer the reader to Feigelson \& Montmerle (1999), Güdel (2004), and Feigelson et al. (2007). XMM-Newton and Chandra penetrate dense molecular envelopes providing unequaled information on high-energy processes. A recent, large survey of the Orion Nebula Cluster (ONC) with Chandra (the COUP project, Getman et al. 2005) has monitored approximately 1400 young stars in a $17^{\prime} \times 17^{\prime}$ field around the Trapezium. This study has provided unprecedented information on the ONC stellar population and the evolution of X-ray production. For example, Preibisch et al. (2005) concluded that a significant correlation between rotation and X-ray luminosity consistently found in main-sequence stars is absent in the ONC sample, arguing for a different magnetic dynamo process or a very different internal structure of these youngest stars. X-ray radiation was found to be largely variable, with numerous flares contributing significantly to the overall X-ray production (Wolk et al. 2005). We refer the interested reader to a special volume of papers from this study (Getman et al. 2005 and associated papers), or to a summary provided by Feigelson et al. (2007).

The ONC represents the so-called "clustered mode" of star formation in which hundreds to thousands of low-mass stars form around massive O-type stars such as those in the Trapezium. Such environments produce extreme stellar densities, and the presence of hot stars has a decisive impact on star formation. UV radiation and the winds of the central $\mathrm{O}$ stars have a deteriorating effect on the molecular environment, the pre-stellar cores and the circumstellar disks by ionizing and dispersing the molecular material, prematurely altering the accretion environment (O'Dell 1998) and eventually halting the star formation process altogether (Dolan \& Mathieu 2001). The ionized H II Orion Nebula is the most vivid expression of this environment.

Star formation occurs, however, also in relatively unspectacular low-mass star forming regions devoid of the influence of radiation and outflow-related effects from luminous $\mathrm{O}$ stars. These so-called "dark clouds" typically form a smaller number of stars and often do so in a "dispersed" or "isolated mode" in which stars or very small groups of stars form in relative isolation. Stars form in a similar manner also in high-mass star formation regions, outside the clusters and at larger distances from the central OB stars (Carpenter 2000).

The closest and best-studied large, low-mass star formation region is the Taurus Molecular Cloud (TMC) complex that extends to the adjacent Auriga Clouds. TMC has served as a testbed for low-mass star-formation theory and has provided some of the best examples of phenomenology relevant to star formation. There are only a few Herbig Ae/Be stars in this vast region (V892 Tau and AB Aur in our survey). The rest are predominantly sub-solar in mass although there have been claims that a substantial population of non-accreting B stars may be members of the Taurus population (Walter \& Boyd 1991), in agreement with expectations from the initial mass function. Owing to the near-absence of massive stars and their mechanical/radiative effects, the molecular gas is still present in large amounts $\left(M_{\text {cloud }} \approx 3 \times 10^{4} M_{\odot}\right.$, Ungerechts \& Thaddeus 1987$)$ much in excess of the total mass in stars already formed $\left(M_{\text {stars }}\right.$ of order $2 \times 10^{2} M_{\odot}$ ).

The Taurus region is quite large (some 10-15 degrees in diameter, corresponding to about $25-35 \mathrm{pc}$ at a distance of $140 \mathrm{pc}$ ), making comprehensive studies of the entire population difficult. We have initiated a large X-ray study of the TMC with the XMM-Newton X-ray observatory, concentrating on the denser cloud areas that contain the majority of the TMC stellar population. The emphasis of this XMM-Newton Extended Survey of the Taurus Molecular Cloud (XEST henceforth) is on a wide-field sampling rather than on long exposures. It goes 
systematically deeper by about an order of magnitude than previous Taurus X-ray surveys. While it is less sensitive than the COUP survey, it surveys an area about 60 times larger $(\approx 5$ square degrees), which is required because of the low surface density of Taurus members. The survey is accompanied by a deep, largefield optical survey with the Canada-France-Hawaii Telescope (CFHT) and a mid-infrared survey of the entire cloud complex with the Spitzer Space Telescope (Padgett et al. 2007). These latter surveys will be presented separately (see summary of all three surveys in Güdel et al. 2007a). All studies combined provide an unsurpassed database for the nearest major star-forming cloud complex.

The purpose of the present paper is to give an introduction to the XEST project, to present an overview of the surveyed stellar sample, and to discuss the strategies followed during the data reduction and analysis. The detailed results are described in a series of associated papers.

The outline of this paper is as follows. We discuss some pivotal properties of the TMC in Sect. 2, including results from previous studies that support the key role of this region for further star-formation studies. Section 3 introduces the TMC X-ray survey, and Sect. 4 presents the data reduction and analysis strategies. Section 5 contains an overview of the basic results, presenting detected and undetected stellar populations, accompanied by comprehensive tabulations of fundamental properties of all surveyed objects and spectral X-ray results from the detections. We summarize overall statistical properties of XEST in Sect. 6.

The subsequent series of papers related to this survey discusses X-ray properties in the context of stellar class and accretion (Telleschi et al. 2007a), rotation-activity relations (Briggs et al. 2007), a dedicated study of the L1495E subsample (Silva et al., in preparation), correlated behavior of X-rays and optical/ultraviolet emission (Audard et al. 2007), X-rays from jetdriving stars in TMC (Güdel et al. 2007b), high-resolution X-ray spectroscopy of classical and weak-line T Tau stars (Telleschi et al. 2007b), X-rays from brown dwarfs (Grosso et al. 2007a), a $U$-band survey of brown dwarfs with the XMM-Newton Optical Monitor (Grosso et al. 2007b), light-curve variability studies (Stelzer et al. 2007), interpretation of X-ray flares (Franciosini et al. 2007), an investigation of statistical fluctuations in X-ray light curves (Arzner et al. 2007a), an investigation of spectral parameters in extremely faint sources (Arzner et al. 2007b), an analysis of the gas-to-dust ratio in IRAS 04158+2805 (Glauser et al. 2007), a study of the gas-to-dust ratios in the TMC in general (Glauser et al., in preparation), a case study of the accreting, prototypical CTTS T Tau (Güdel et al. 2007c), a study of the supposedly single Herbig star in TMC, AB Aurigae (Telleschi et al. 2007c), and a search for new TMC members based on nearinfrared and X-ray properties of field sources detected in XEST (Scelsi et al. 2007).

\section{The Taurus molecular cloud complex}

At a distance around 140 pc (e.g., Loinard et al. 2005; Kenyon et al. 1994), the TMC is the nearest large star formation region and reveals characteristics that make it ideal for detailed physical studies. One of the most notable properties of TMC in this regard is its structure in which several loosely associated but otherwise rather isolated molecular cores each produce one or only a few low-mass stars, different from the much denser cores in $\rho$ Oph or in Orion. TMC features a low stellar density of only 110 stars $\mathrm{pc}^{-3}$ (e.g., Luhman 2000). Strong mutual influence due to outflows, jets, or gravitational effects are therefore minimized. Strong stellar winds and ionizing UV radiation are mostly absent in TMC because there are no $\mathrm{O}$ stars and only very few $\mathrm{B}$ and $\mathrm{A}$ stars (Walter \& Boyd 1991). Further, most stars in TMC are subject to relatively modest extinction, providing access to a broad spectrum of stars at all evolutionary stages from Class 0 sources to near-zero age main-sequence T Tau stars. TMC has also become of central interest for the study of substellar objects, in particular brown dwarfs, with regard to their evolutionary history and their spatial distribution and dispersal (Briceño et al. 2002; Guieu et al. 2006).

TMC has figured prominently in star-formation studies at all wavelengths. It has provided the best-characterized sample of classical and weak-line T Tau stars (CTTS and WTTS, respectively, or "Class II" and "Class III" objects in the infrared classification - Kenyon \& Hartmann 1995); most of our current picture of low-density star formation is indeed based on IRAS studies of Taurus (Strom et al. 1989; Weaver \& Jones 1992). Among the key results from TMC studies as listed in Kenyon \& Hartmann (1995) figure the following: i) more than $50 \%$ of the TMC objects have IR excess beyond the photospheric contribution, correlating with other activity indicators ( $\mathrm{H} \alpha$, UV excess etc.) and indicating the presence of warm circumstellar material predominantly in the form of envelopes for Class I protostars and circumstellar disks for Class II stars. ii) Class III sources (mostly to be identified with WTTS) are distinctly different from Class I-II objects by not revealing optically thick disks or signatures of accretion. iii) Star formation has been ongoing at a similar level during the past 1-2 Myr, with the Class-I protostars having ages of typically $0.1-0.2$ Myr. iv) There is clear support for an evolutionary sequence Class $\mathrm{I} \rightarrow \mathrm{II} \rightarrow \mathrm{III}$, although there is little luminosity evolution along this sequence, indicating different evolutionary speeds for different objects (see also Hartmann 2002). The infall time scale is a few times $10^{5}$ yrs, while the disk phase amounts to a few times $10^{6}$ yrs. An evolutionary scenario is also suggested from the different spatial distribution of Class 0/I stars vs. CTTS/WTTS with respect to the gas distribution: the former classes are still within the boundaries of the high density gas, while the latter are found in regions of lower density (Palla \& Stahler 2001).

TMC has also been well-studied at millimeter wavelengths. This region has better high-resolution molecular line maps than any other star-forming region (Onishi et al. 2002). Most of the higher-mass CTTS and Class I protostars have been surveyed by millimeter interferometers for molecular line emission from disks (Dutrey et al. 1996; Ohashi et al. 1996, etc.) and many detailed studies of individual sources have been published (e.g., Qi et al. 2003; Duchêne et al. 2003). A variety of millimeter continuum observations of these sources have enabled studies of cold disk frequency into substellar mass ranges and raised the possibility of grain growth within circumstellar disks (Scholz et al. 2006; Rodman et al. 2006; Wolf et al. 2003; Kitamura et al. 2002 , etc.). Due to its proximity and easy accessibility to northern hemisphere radio telescopes, the TMC has served as a template for millimeter studies of young stellar objects.

Although TMC has been regarded, together with the $\rho$ Oph dark cloud, as the prototypical low-mass star-forming region, a few apparent peculiarities deserve to be mentioned. TMC contains an anomalous number of binaries (Ghez et al. 1993; Duchêne 1999), compared with other SFRs (e.g., Orion) or with field stars. In TMC, about two thirds of all members are bound in multiple systems, with an average separation of about $0.3^{\prime \prime}$ (e.g., Leinert et al. 1993; Mathieu 1994; Simon et al. 1995; Duchêne et al. 1999; White \& Ghez 2001; Hartigan \& Kenyon 2003). Petr et al. (1998) reported, at the $96 \%$ confidence level, a three times higher binary occurrence in TMC compared to Orion for 
component separations in the range of 63-225 AU. Also, TMC cloud cores are comparatively small and of low mass, at least when compared with cores in Orion or Perseus (Kun 1998).

Initially, TMC was also found to be deficient of lowest-mass stars and brown dwarfs, with a mass distribution significantly enriched in $0.5-1 \mathrm{M}_{\odot}$ stars, compared to Orion samples (Luhman 2000; Briceño et al. 2002). It was speculated that the formation of brown dwarfs could be different in the low-density environment of TMC compared to the dense packing of stars in Orion. However, a new wide-field search for low-mass TMC members now indicates that there is no BD deficit in Taurus (Guieu et al. 2006).

In X-rays, Taurus has again played a key role in our understanding of high-energy processes and circumstellar magnetic fields around pre-main sequence stars. Taurus X-ray studies provided the first detailed view of the X-ray behavior of T Tauri stars (Feigelson \& Kriss 1981; Feigelson \& DeCampli 1981; Walter \& Kuhi 1981). Among the key surveys of the entire region are those by Feigelson et al. (1987), Walter et al. (1988), Bouvier (1990), Strom et al. (1990), Damiani \& Micela (1995), Damiani et al. (1995), based on Einstein Observatory observations, and the work by Strom \& Strom (1994), Neuhäuser et al. (1995), and Stelzer \& Neuhäuser (2001) based on ROSAT. These surveys have characterized the overall luminosity behavior of TTS and studied the dependence of X-ray activity on rotation. Bally et al. (2003) and Favata et al. (2003) reported, respectively, Chandra and XMM-Newton studies of the L1551 cloud region (the longer exposure from XMM-Newton will be included in our survey; the Chandra exposure contains no additional sources).

But again, for reasons very poorly understood, TMC differs from other SFRs significantly also with regard to X-ray properties. Whereas no X-ray activity-rotation correlation (analogous to that in main-sequence stars) is found for samples in the Orion star-forming regions, perhaps suggesting that all stars are in a saturated state (Flaccomio et al. 2003a; Preibisch et al. 2005), the X-ray activity in TMC stars has been reported to decrease for increasing rotation period (e.g., Neuhäuser et al. 1995; Damiani \& Micela 1995; Stelzer \& Neuhäuser 2001). Also, claims have been made that the X-ray behavior of TMC CTTS and WTTS is significantly different, CTTS being less luminous than WTTS (Strom \& Strom 1994; Damiani et al. 1995; Neuhäuser et al. 1995; Stelzer \& Neuhäuser 2001). This contrasts with other star-forming regions (Flaccomio et al. 2000; Preibisch \& Zinnecker 2001), but recent reports reveal a similar segregation also for Orion and some other SFRs (Flaccomio et al. 2003b; Preibisch et al. 2005). Some of these discrepancies may be due to selection and detection bias (e.g., WTTS are predominantly identified in X-ray studies, in contrast to CTTS), but also to incomplete samples due to high detection limits. Issues related to rotation and selection biases will be discussed in detail in one of the companion papers, see Briggs et al. (2007).

\section{The XEST project}

\subsection{Scientific goals}

The scientific goals of XEST are:

- To collect X-ray spectra and light curves from a statistically meaningful sample of TMC objects, and to characterize them in terms of X-ray emission measure distributions, temperatures, X-ray luminosities, and variability.

- To interpret X-ray emission in the context of other stellar properties such as rotation, mass, and radius.
- To investigate changes in the X-ray behavior as a young stellar object evolves.

- To obtain a census of X-ray emitting objects at the stellar mass limit and in the substellar regime (brown dwarfs = BDs).

- To study in what sense the stellar environment (circumstellar disks, jets, accretion) influences the X-ray production, and vice versa.

- To study the gas-to-dust ratio in the circumstellar environment, making use of extinction measurements obtained in the optical or near-infrared.

- To assess the role of flares in coronal heating, and to study flare characteristics in their own right.

- To search for new, hitherto unrecognized, TMC members.

The outstanding characteristics of the survey are its sensitivity and its energy resolution, covering a large fraction of the most densely populated regions of the Taurus clouds. It is by far the largest-area Taurus survey at this sensitivity. It reaches sensitivities about ten times better than previous surveys such as those conducted with ROSAT (Neuhäuser et al. 1995; Stelzer \& Neuhäuser 2001) and therefore permits a systematic study of the lowest-mass Taurus members. Because of the harder band used for the XEST project (compared to ROSAT's 0.1-2.4 keV band), XEST also detects several deeply embedded Taurus protostars systematically for the first time, while they remained undetected in softer-band surveys. Given the achieved sensitivity, the XEST project accesses the known TMC population in the surveyed fields nearly completely, thus suppressing potential bias that previous surveys may have been subject to. Energy resolution permits a detailed description of the plasma properties together with the measurement of the absorbing gas columns that are located predominantly in the TMC clouds themselves, and even in the immediate circumstellar environment in the case of strongly absorbed objects.

\subsection{Instruments and exposures}

$\mathrm{XEST}$ is a wide-field $\mathrm{X}$-ray survey obtained with the $X M M$ Newton X-ray observatory, principally based on combined CCD camera exposures, but complemented by exposures of a few bright targets with the reflection grating spectrometers and fields observed with the optical monitor. A few complementary observations obtained with the Chandra X-Ray Observatory have also been included.

XMM-Newton (Jansen et al. 2001) orbits the Earth in a $48 \mathrm{hr}$ orbit, permitting long, uninterrupted exposures. It carries three high-throughput telescopes feeding two suites of X-ray instruments, and also features an optical telescope. A short description of the instruments and the chosen setups follows. ${ }^{1}$

i) The European Photon Imaging Cameras (EPICs) are three CCD-based X-ray cameras, one per telescope, that operate entirely independently. Two cameras are of the MOS type (Turner et al. 2001), and one is of the PN type (Strüder et al. 2001). The circular field of view of each camera has a diameter of $30^{\prime}$, and the three telescopes are nearly co-aligned. The PN camera provides most counts because the beams to the MOS cameras are intersected by the reflection gratings that consume approximately half of the X-ray flux. The MOS PSF has a full-width-athalf-maximum (FWHM) of about 4-5", while the half-energy width (HEW) is larger, amounting to some 13-15". The cameras provide pixel sizes of $1.1^{\prime \prime}(\mathrm{MOS})$ or $4^{\prime \prime}(\mathrm{PN})$. The energy

\footnotetext{
1 For further details, see XMM-Newton User's Handbook (http://xmm.vilspa.esa.es/)
} 
resolution is approximately $E / \Delta E \approx 45$ at $6.7 \mathrm{keV}$, scaling as $E^{-1 / 2}$. To prevent the spectra from being altered by optical load, various filters can be inserted.

We usually applied the medium filter to all EPIC cameras, providing a combined on-axis effective area for all three cameras of $\approx 1800 \mathrm{~cm}^{2}$ at $1.5 \mathrm{keV}$. Exceptions were exposure XEST26 for which the thick filter was used for the two MOS cameras to suppress optical load from the Herbig star AB Aur (the PN camera was not recording), and exposures XEST-27 and XEST28 for which the thick filter was used for each EPIC camera. All cameras were operated in full window mode, i.e., the entire field of view was exposed, confining the time resolution to $2.6 \mathrm{~s}$ for the MOS and to $73 \mathrm{~ms}$ for the PN camera, entirely appropriate for the moderate brightness of our sources. The only exceptions here were the fields around V773 Tau (XEST-20) where MOS2 was operated in the small-window mode, and the field of T Tau (XEST-01) for which MOS1 was operated in small-window mode, and MOS2 was operated in large-window mode. These modes confine the central CCD to $100 \times 100$ and $300 \times 300$ pixels, respectively, compared to $600 \times 600$ pixels for the full window mode, but avoiding pile-up during potential large flares.

ii) Two Reflection Grating Spectrometers (RGS, den Herder et al. 2001) are each fed by about $50 \%$ of the light of one of the telescopes. The dispersed spectra comprise the wavelength range from 5 to $35 \AA$, although in each spectrometer, one of the nine chips has failed earlier in the mission, leaving a gap in the 10.613.8 $\AA$ range for RGS1 and in the 20.0-24.1 $\AA$ range for RGS2. The first-order spectral resolution is approximately $60-70 \mathrm{~m} \AA$ $(F W H M)$ at any wavelength, corresponding to $\lambda / \Delta \lambda \approx 300$ at $\lambda=20 \AA$. The combined first-order effective area is $\approx 115 \mathrm{~cm}^{2}$ at $15 \AA$.

iii) The Optical Monitor (OM, Mason et al. 2001) is a microchannel-plate enhanced CCD camera fed by a $30 \mathrm{~cm}$ mirror co-aligned with the EPIC cameras. Its square field of view is, however, somewhat smaller than the latters', amounting to $17^{\prime}$ side-length, i.e., a radius of $12^{\prime}$ along the diagonal. The OM can be used in combination with various filters. Filter use may be dictated by the brightness of the stars in the field of view. We obtained most of the exposures with the $U$ band filter inserted, given the diagnostic importance of this band for both chromospheric flare processes and accretion mechanisms. In a few cases, another near-UV filter was used (UVW1: 2500-3500 $⿱$ A; UVW2: 1700-2500 $\AA$ ) to suppress excessive optical load. The window mode we applied essentially observed the entire field of view. The detector area is split up into a series of windows, some of which are sequentially exposed and read out. The exposure time for one of these frames determines the time resolution for any source within the window. This time resolution is of order 1000-2000 s but varies within any given XEST OM field, and is also not identical between different XEST fields. A detailed presentation is given by Audard et al. (2007). Additionally, if a bright source was present on-axis, the central $10.5^{\prime \prime} \times 10.5^{\prime \prime}$ of the OM field were exposed within a high-time resolution window that collects photons with a time resolution of $0.5 \mathrm{~s}$.

Our initial project collected 19 fields in the TMC coherently with exposure times of about $30 \mathrm{ks}$ each. The effective exposure time varied between $31.2 \mathrm{ks}$ and $41.9 \mathrm{ks}$. Relevant information is given in Table 1. The start and end times of the observations as listed in Table 1 are the times of the earliest and latest recording of any of the three EPIC cameras, and the quoted exposure time is the difference between end and start times. The pointing coordinates are the nominal boresight coordinates, which are not identical with the coordinates of the center of the EPIC fields of view, the latter being slightly misaligned (e.g., to avoid the PN CCD edges crossing the exact field center). The last column gives the filter used for the OM ( $U$ band, or one of the ultraviolet filters, $U V W 1$ or $U V W 2$ ). A letter $F$ indicates that the central star was recorded in fast mode (see Audard et al. 2007).

The fields of view were selected such that they cover the densest concentrations of $\mathrm{CO}$ gas, which also show the strongest accumulations of TMC stellar and substellar members. We complemented this sample with exposures that were obtained as part of separate projects, partly retrieved from the XMM-Newton archive (marked in Table 1); in all of these observations very similar instrument setups were used, although in most cases the exposure times were longer and the strategies for the use of the OM were different. The eight additional fields are: 1) L1495 centered at V410 Tau (XEST exposures 23 and 24, see Table 1; two exposures totaling $117.9 \mathrm{ks}$; PI F. Walter); 2) L1551, centered near L1551 IRS-5 (XEST exposure 22, 56.9 ks, PI F. Favata); 3) a field centered at AA Tau (eight exposures of 16-19.5 ks each of which we use only one [XEST exposure 25] that reveals the strongly variable AA Tau X-ray source in an average state - details in Grosso et al., in preparation; PI J. Bouvier); 4) a field centered at HD 283572 (XEST exposure 21, $46.2 \mathrm{ks}$, PI R. Pallavicini); 5) a field around BP Tau (XEST exposure 28, 131.3 ks, PI J. Schmitt); 6) a field around SU Aur (XEST exposure 26, $129.6 \mathrm{ks}$, PI R. Pallavicini); 7) a field around $\zeta$ Per (XEST exposure 27, $43.4 \mathrm{ks}$, PI W. Waldron); 8) a field around $\mathrm{T}$ Tau (XEST exposure 1, $83.0 \mathrm{ks}$ - a special discussion of this observation is given by Güdel et al. (2007c); PI M. Güdel). The entire survey includes approximately 5 sq. degrees in total, with a total exposure time of about 1.3 Ms. The spatial coverage is illustrated in Fig. 1.

We have included a few exposures obtained by Chandra that contain important objects outside the XMM-Newton coverage, or faint objects not detected by XMM-Newton but detected by Chandra. Objects that were covered by the XEST program (regardless of their detection status) and were not detected in these Chandra exposures will not be reported in our survey tables. The additional fields are defined in Table 2 . Note that different detectors were used for these observations. The entire fields of view were scanned for TMC objects that add to the project, but no systematic survey of all X-ray sources in the fields was performed. The exposure around GV Tau does not contain new sources beyond those detected in the XMM-Newton field but provides better angular resolution for the study of the origin of the X-rays in this binary system (Güdel et al. 2007b). We also add the strong X-ray detection coincident with HD $28867=$ HR 1442 that has been suspected to be a late-B (non-Herbig) binary member of the TMC (e.g., Walter \& Boyd 1991). This object has been extensively studied by Walter et al. (2003) who found evidence for a G-type companion to one of the B-type components.

The XEST work presented here and in the accompanying papers addresses X-ray properties of the commonly known stellar and substellar population. A considerable number of hitherto unrecognized Taurus members may be present in the survey, e.g., deeply embedded sources or extincted stars on the far side of the clouds. An attempt to identify some objects of this population has been undertaken by Scelsi et al. (2007).

\section{Strategy and analysis of the TMC survey}

Our XMM-Newton data analysis procedure predominantly uses tasks provided in the XMM-Newton Science Analysis Software (SAS) v6.1.0, augmented by procedures in FTOOLS, and is tied together in Perl scripts developed at the Paul Scherrer 
Table 1. Observing log of XEST program.

\begin{tabular}{|c|c|c|c|c|c|c|c|}
\hline $\begin{array}{r}\text { Exposure } \\
\# \\
\end{array}$ & ObsID $^{a}$ & $\begin{array}{r}\mathrm{RA}(\mathrm{J} 2000.0)^{b} \\
\mathrm{~h} \mathrm{~m} \quad \mathrm{~s}\end{array}$ & $\begin{array}{r}(\mathrm{J} 2000.0)^{b} \\
\text { deg }{ }^{\prime} \prime \prime\end{array}$ & $\begin{array}{r}\text { Start time } \\
\mathrm{y}-\mathrm{m}-\mathrm{d} h: \mathrm{m}: \mathrm{s}\end{array}$ & $\begin{array}{r}\text { Stop time } \\
\text { y-m-d h:m:s }\end{array}$ & $\begin{array}{r}\text { Exposure } \\
\text { time (s) }\end{array}$ & $\begin{array}{l}\text { OM mode, } \\
\text { OM filter }^{d}\end{array}$ \\
\hline $1^{e}$ & 0301500101 & 042159.4 & 193206 & $2005-08-15$ 13:52:13 & $2005-08-16$ 12:55:22 & 82989 & $F, U V W 1$ \\
\hline 2 & 0203540201 & 042719.6 & 260925 & 2004-08-17 06:08:10 & 2004-08-17 17:32:46 & 41076 & $U$ \\
\hline 3 & 0203540301 & 043218.9 & 242228 & 2004-08-22 06:45:22 & 2004-08-22 16:37:04 & 35502 & $F, U$ \\
\hline 4 & 0203540401 & 043334.4 & 242108 & $2005-02-2101: 18: 21$ & $2005-02-21 \quad 10: 31: 17$ & 33176 & $F, U$ \\
\hline 5 & 0203540501 & 043934.9 & 254146 & $2005-02-21 \quad 11: 25: 51$ & $2005-02-2120: 13: 47$ & 31676 & $U$ \\
\hline 6 & 0203540601 & 040442.9 & 261856 & 2004-08-25 20:53:54 & $2004-08-26$ 05:41:50 & 31676 & $U$ \\
\hline 7 & 0203540701 & 044112.5 & 254637 & $2005-02-24$ 20:28:00 & $2005-02-25$ 05:15:58 & 31678 & $U$ \\
\hline 8 & 0203540801 & 043552.9 & 225423 & 2004-08-26 06:36:23 & 2004-08-26 18:10:59 & 41676 & $F, U$ \\
\hline 9 & 0203540901 & 043555.1 & 223924 & 2005-02-25 08:38:53 & $2005-02-25$ 17:26:50 & 31677 & $U$ \\
\hline 10 & 0203542201 & 044220.9 & 252035 & 2005-03-05 05:56:38 & 2005-03-05 14:44:30 & 31672 & $F, U$ \\
\hline 11 & 0203541101 & 042151.1 & 265733 & 2004-08-18 06:44:13 & 2004-08-18 18:21:54 & 41861 & $U$ \\
\hline 12 & 0203542101 & 043517.4 & 241500 & 2005-03-04 20:22:29 & 2005-03-05 05:01:54 & 31165 & $F, U$ \\
\hline 13 & 0203541301 & 042952.0 & 243647 & 2004-08-25 11:11:23 & 2004-08-25 19:59:19 & 31676 & $U$ \\
\hline 14 & 0203541401 & 043030.6 & 260214 & 2005-02-09 03:01:02 & 2005-02-09 12:30:38 & 34176 & $U$ \\
\hline 15 & 0203541501 & 042942.4 & 263251 & 2005-02-09 13:12:40 & 2005-02-09 22:38:18 & 33938 & $F, U$ \\
\hline 16 & 0203541601 & 041943.0 & 271334 & 2004-08-21 05:53:24 & $2004-08-21 \quad 17: 11: 22$ & 40678 & - \\
\hline 17 & 0203541701 & 043321.2 & 225241 & 2005-02-11 01:19:56 & 2005-02-11 10:07:50 & 31674 & $U$ \\
\hline 18 & 0203541801 & 043354.7 & 261328 & 2004-08-13 21:48:05 & 2004-08-14 06:36:03 & 31678 & $F, U$ \\
\hline 19 & 0203541901 & 043243.0 & 255232 & 2004-08-14 07:18:05 & 2004-08-14 17:50:41 & 37956 & $F, U$ \\
\hline 20 & 0203542001 & 041412.9 & 281212 & 2004-09-12 07:04:43 & 2004-09-12 15:52:37 & 31674 & $F, U$ \\
\hline $21^{e}$ & 0101440701 & 042159.0 & 281808 & 2000-09-05 02:57:44 & 2000-09-05 15:47:55 & 46211 & - \\
\hline $22^{e}$ & 0109060301 & 043139.0 & 181000 & 2000-09-09 18:29:39 & 2000-09-10 10:18:12 & 56913 & $U V W 2$ \\
\hline $23^{e}$ & 0086360301 & 041831.2 & 282716 & 2001-03-11 12:46:45 & 2001-03-12 09:13:24 & 73599 & $(U V W 2)^{f}$ \\
\hline $24^{e}$ & 0086360401 & 041831.2 & 282716 & 2001-03-12 09:29:38 & 2001-03-12 21:47:51 & 44293 & $(U V W 2)$ \\
\hline $25^{e}$ & $0152680201^{g}$ & 043455.5 & 242854 & 2003-02-14 02:18:48 & 2003-02-14 07:01:32 & 16964 & UVW2 \\
\hline $26^{e}$ & 0101440801 & 045559.0 & 303402 & 2001-09-21 01:34:17 & 2001-09-22 13:34:31 & 129614 & - \\
\hline $27^{e}$ & 0201550201 & 035407.9 & 315301 & 2004-02-13 21:45:10 & 2004-02-14 09:48:07 & 43377 & - \\
\hline $28^{e}$ & 0200370101 & 041915.8 & 290627 & 2004-08-15 06:14:30 & 2004-08-16 18:42:57 & 131307 & UVW1 \\
\hline
\end{tabular}

${ }^{a}$ XMM-Newton observation identification number.

${ }^{b}$ Nominal boresight coordinates.

${ }^{c}$ Earliest respectively latest time of recording for any EPIC camera is reported.

${ }^{d} F$ = fast + imaging mode (only imaging otherwise); for filter name, see XMM-Newton User's Handbook (http://xmm.vilspa.esa.es/).

${ }^{e}$ Observation from separate projects.

${ }^{f}$ The OM was recording only for short time intervals.

${ }^{g}$ Eight different observations of $\approx 16-19$ ks $(\mathrm{PN})$ each around AA Tau, 2003-02-14 - 2003-02-28, ObsID = 0152680201-901.

Table 2. Complementary Chandra observations.

\begin{tabular}{|c|c|c|c|c|c|c|c|c|}
\hline Exposure & Main & $\begin{array}{l}\text { Obs } \\
\mathrm{ID}^{a}\end{array}$ & $\mathrm{RA}(\mathrm{J} 2000.0)^{b}$ & $\begin{array}{c}\delta(\mathrm{J} 2000.0)^{b} \\
\operatorname{deg}^{\prime},{ }^{\prime}\end{array}$ & $\begin{array}{r}\text { Start time } \\
\mathrm{V}-\mathrm{m}-\mathrm{d} \cdot \mathrm{h} \cdot \mathrm{m} \cdot \mathrm{s}\end{array}$ & Stop time & Exposure & Instr. \\
\hline$\frac{\pi}{\mathrm{C} 1}$ & V410 Tau & 3364 & $\begin{array}{l}\text { II III s } \\
041834.6\end{array}$ & 282247 & $\begin{array}{r}y-111-\mathrm{u} 11.11 . \mathrm{s} \\
2002-03-0706: 16: 32\end{array}$ & $\begin{array}{r}y-11-\mathrm{u} 1 \mathrm{~m} . \mathrm{s} \\
2002-03-0711: 45: 24\end{array}$ & 17734 & $\overline{\mathrm{ACIS}-\mathrm{S}}$ \\
\hline $\mathrm{C} 2$ & FS Tau & 4488 & 042200.2 & 265807 & $2003-11-08$ 12:57:58 & $2003-11-08$ 21:56:34 & 29674 & ACIS-S \\
\hline $\mathrm{C} 3$ & DG Tau & 4487 & 042702.3 & 260456 & 2004-01-11 02:58:51 & 2004-01-11 11:52:21 & 29717 & ACIS-S \\
\hline $\mathrm{C} 4$ & GV Tau & 4498 & 042923.3 & 243244 & $2003-12-28$ 08:56:57 & $2003-12-28 \quad 16: 37: 59$ & 24650 & ACIS-I \\
\hline $\mathrm{C} 5$ & HR 1442 & 612 & 043333.1 & 180115 & 2000-09-11 17:53:33 & 2000-09-11 19:41:57 & 4679 & HRC-I \\
\hline C6 & L1527 & 2563 & 043952.7 & 260305 & 2002-12-06 08:30:12 & 2002-12-06 14:21:15 & 19317 & ACIS-I \\
\hline
\end{tabular}

${ }^{a}$ Chandra observation identification number. ${ }^{b}$ Nominal boresight coordinates.

Institut. Source identification was based on procedures involving maximum-likelihood algorithms and wavelet analysis. In order to optimize detection of faint sources, periods of high background radiation levels due to local particles were cut out. All programs named below can be assumed to be SAS tasks unless otherwise noted.

\subsection{Creation of event lists and event selection}

An event list was produced for each active EPIC instrument from the original data files using the standard SAS procedures EPCHAIN and EMCHAIN. MOS event lists were filtered to include only events flagged as 'good' and with patterns 0-12 (Kirsch 2006). PN event lists were filtered using the selection expression (FLAG \& 0xfb0825) =0, which rejects events with patterns $>12$, close to CCD windows or on-board bad pixels, in spoiled frames, or outside the field of view, but retains events on or next to offset columns, close to bright pixels or dead pixels. Remaining bright pixels, columns and partial columns observed in images at energies $>300 \mathrm{eV}$ were further added to the bad pixel list and rejected.

Source detection was performed in three energy bands: soft (500-2000 eV), hard (2001-7300 eV) and full (500-7300 eV). These were chosen with consideration of the energy-dependent sensitivity of the EPIC instruments, the expected spectra of our sources of interest, the energies of strong fluorescent spectral features from the detectors (particularly $\mathrm{Ni}, \mathrm{Cu}$ and $\mathrm{Zn} \mathrm{K} \alpha$ at energies 7.3-9 keV), and compatibility of count-rates and fluxes 


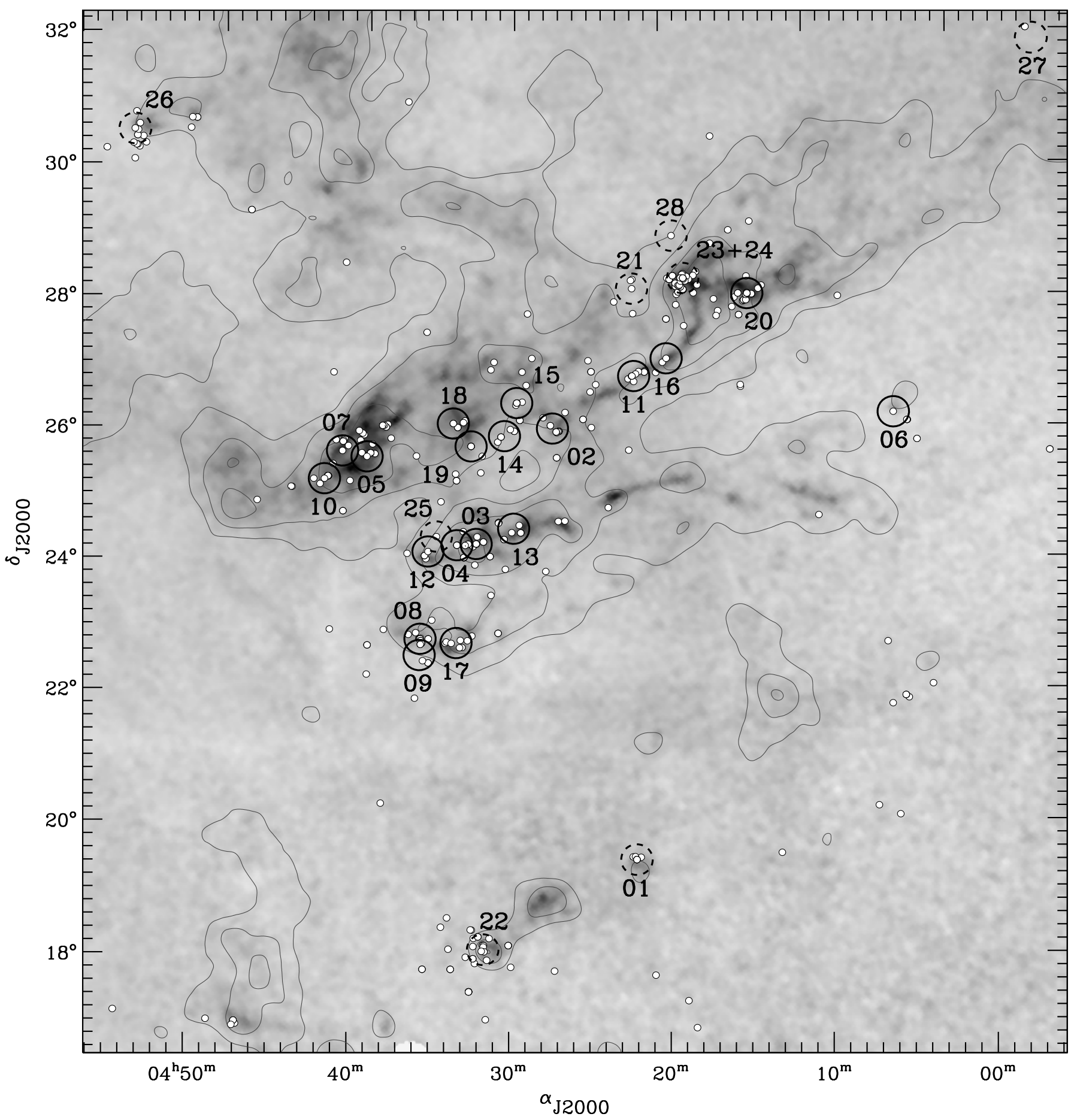

Fig. 1. Map of the TMC region (oriented in RA - dec, north is up, east to the left). The grayscale background map is an extinction $\left(A_{\mathrm{V}}\right)$ map from Dobashi et al. (2005). Contours show the CO emission (Dame et al. 1987). The large (0.5 degrees diameter) circles show the fields of view of the XMM-Newton survey (the dashed circles marking fields from separate projects also used for the survey). Small white dots mark the positions of individual young TMC members. The labels correspond to the XEST exposure numbers in Table 1. Note the outlying XMM-Newton fields XEST-26 around SU Aur (NE corner), XEST-27 around $\zeta$ Per (NW corner), XEST-01 around T Tau, and XEST-22 around L1551 (the latter two at the southern border of the figure).

derived therefrom with those quoted using previous and current instruments for X-ray astronomy. We give an outline of the source detection procedure here and describe each stage in more detail below.

For each EPIC instrument and energy band:

- Define good time intervals that reject times of high background rate.
- Extract an image using good time intervals.

- Generate an exposure map.

- Create a background map.

For each band:

- Mosaic the images of the (up to) three instruments to use the full sensitivity of the combined EPIC detectors. 
- Mosaic the background maps.

- Scale the exposure maps to account for the differences in sensitivity and mosaic.

- Locate candidate sources using a wavelet transform algorithm.

Finally:

- Match candidate sources from all bands and concatenate into a single list.

- Parameterize candidate sources in all bands simultaneously via maximum-likelihood fitting of the point-spread function and reject those with low detection significance.

- Verify by eye and reject obvious spurious detections.

- Calculate upper limits in bands where each source is undetected.

- Calculate and correct any systematic offset of the Xray source positions from the positions of near-infrared counterparts.

\subsection{Definition of good time intervals}

In almost every observation the background count-rate was highly variable, by factors up to several hundred ${ }^{2}$. To optimize sensitivity to the detection of faint sources, it was necessary to exclude intervals of highest background count-rate. Good time intervals (GTIs) were defined independently for each instrument and energy band as follows.

A light curve was extracted of the whole active detector area (excluding bright sources, as described in the creation of background maps, below) with a binning of $52 \mathrm{~s}$, scaled to a 1 sq. arcsec area, and ordered by increasing count-rate. Its cumulative distribution was made and the source count-rate required to give a $4 \sigma$ detection in a circular region of $15 \operatorname{arcsec}^{3}$ was calculated for each bin, and the minimum was found. This defined the maximum background count-rate accepted, and hence the good time intervals (see Fig. 2). In some observations, the source count-rate required for a $4 \sigma$ detection is a factor three higher than would be expected for a 30-ks observation performed with background at the quiescent level. The total accepted good exposure times for the full energy band for each instrument in each observation are listed in Table 3.

\subsection{Extraction of images}

An image was created for each instrument and each energy band, in sky coordinates with pixel size $2^{\prime \prime} \times 2^{\prime \prime}$, using data in good time intervals only. The intrinsic pixel sizes of the PN and MOS detectors are $4^{\prime \prime}$ and $1.1^{\prime \prime}$, respectively. In processing, a randomization of the event position within a pixel is performed to avoid fixed pattern noise. $2^{\prime \prime}$ was simply found to provide much better separation of close sources than $4^{\prime \prime}$.

For each energy band, the images from the three detectors were mosaicked to use the full sensitivity of the combined EPIC cameras. The relative astrometry of the EPIC detectors is less than $1.5^{\prime \prime}$, so we applied no offset between the images.

An exposure map was generated for each image using EEXPMAP. A summed exposure map was also produced for each energy band, but first MOS exposure maps were scaled

2 The EPIC background is described at http://xmm.vilspa.esa.es/external/xmm_sw_cal/ background/index. shtml; see also Read \& Ponman (2003).

3 This encloses approximately 68 per cent of source counts and is the area used by the SAS source parameterization task EMLDETECT.
XEST-16 500-7300 eV pn background light-curve and GTIs
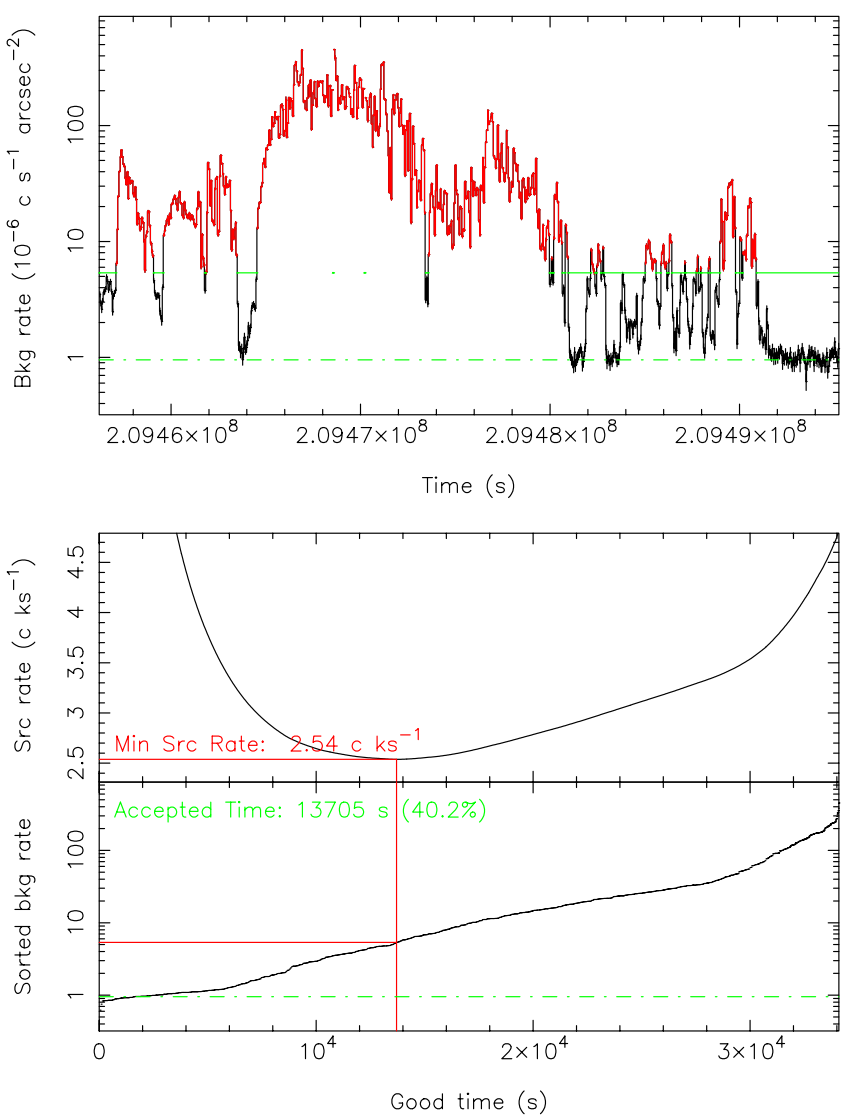

Fig. 2. The selection of good time intervals to reject times affected by high background and so optimize sensitivity to the detection of faint sources. The background count rate per unit area in the $0.5-7.3 \mathrm{keV}$ energy band is shown in the top panel. The expected quiescent background level in this band is marked by the green dot-dashed lines. The bottom panel shows the background count-rate sorted in ascending order. The middle panel shows as a function of accumulated good exposure time the minimum source count-rate that will give a $4 \sigma$ detection against the accumulated background in a circular source extraction region of radius $15^{\prime \prime}$, containing 67 per cent of the source counts. The minimum defines the amount of accepted good time, noted in the bottom panel in green in seconds and as a percentage of the total exposure time, and the maximum acceptable background rate, marked in the upper panel by a green solid line. The time bins marked in red in the upper panel are rejected. XEST-16, shown here, was one of the observations worst-affected by high background, but time intervals with background levels more than 100 times the quiescent level occurred in most observations.

to account for the lower sensitivity compared with the PN. The scaling factors used were $0.370,0.345$ and 0.385 in the full, soft and hard bands, respectively: these factors are strictly dependent on the individual source spectrum; the values used are median values found for all sources detected in the first 12 analyzed observations. The count rates resulting from our source detection are therefore approximate $\mathrm{PN}$-equivalent count rates.

\subsection{Creation of background maps}

A background map was created for each image: each instrument and energy band. The basic method was to mask out regions of the image affected by source counts and apply a 2D Gaussian smoothing filter of $\sigma=60^{\prime \prime}$. The background map was produced in three iterations which successively improved 
the source parameterization and hence the mask. We first used the SAS wavelet transform algorithm EWAVELET to locate and approximately parameterize sources. The resultant source list and background map were input into the maximum-likelihood fitting algorithm EMLDETECT to produce an improved background which was input into a second run of EMLDETECT to produce the final background map. The mask, produced using REGION, masked out each source with an elliptical area, whose eccentricity reflected the shape of the point-spread function at the source position and whose size reflected the number of source counts. Smoothing was performed using ASMOOTH. EMLDETECT outputs an image of the parameterized sources, which was masked and smoothed in the same way as the image and subtracted from the background to remove the effect of remaining source counts in the background map. Out-of-time events (OOTEs) in PN images were modeled into the background map. An image of OOTEs was created ${ }^{4}$ and smoothed only along the CCD readout direction, subtracted from the true image before source detection and smoothing, and added to the resulting background map. Where a central MOS CCD observed a bright source in a window mode such that no reliable background level could be assessed from this CCD, the background in that CCD was estimated as 1.15 times the mean background in the other CCDs, as measured from MOS observations in full frame mode lacking a bright central source. For each energy band, we mosaicked the background maps from all EPIC detectors.

\subsection{Location of source candidates}

Source candidates in the mosaicked EPIC image in each energy band were located using two wavelet transform algorithms: the SAS task EWAVELET, and PWXDETECT developed at the Osservatorio Astronomico di Palermo (Damiani et al. 1997). In PWXDETECT the wavelet transform (WT) is obtained from the rate image (image/exposure map) $r(x, y)$ as:

$w(x, y ; a)=\iint g\left(\frac{x-x^{\prime}}{a}, \frac{y-y^{\prime}}{a}\right) r\left(x^{\prime}, y^{\prime}\right) \mathrm{d} x^{\prime} \mathrm{d} y^{\prime}$.

The WT is evaluated at different values of the scale parameter, $a$, of the generating wavelet ${ }^{5}$, going from $2.8^{\prime \prime}$ to $16^{\prime \prime}$ with a logarithmic step of $\sqrt{2}$. This property makes this multiscale WT particularly suited when the PSF varies across the detector image, as well as very effective in detecting extended sources: for the case of the EPIC images, point-like sources are detected typically at $a=8$ " (although faint sources with undersampled PSF may be detected either at low and at high detection scale), while extended sources typically result with $a=16^{\prime \prime}$.

Since sources are defined as local maxima in the WT space, thresholds for detection at a given confidence level must be assessed for each field and each band. This is obtained by means of extensive simulations on summed PN and MOS images containing only background photons. The number of background photons in each simulated PN or MOS image is evaluated from the observed image relevant to that band, instrument and field. To avoid rejecting possible source candidates, unconservative thresholds were adopted so as to give about 150 spurious detections due to background fluctuations, per band and per field.

\footnotetext{
${ }^{4}$ See http://xmm.vilspa.esa.es/sas/new/documentation/ threads/EPIC_OoT.html

5 The wavelet function $g$ adopted in this method is a two-dimensional "Mexican hat": $g(x / a, y / a)=\left(2-r^{2} / a^{2}\right) \mathrm{e}^{-r^{2} / 2 a^{2}} \quad\left(r^{2}=x^{2}+y^{2}\right)$.
}

These simulations were also used to assess the number of false detections expected after maximum likelihood fitting of the candidate sources (see below). EWAVELET uses a similar methodology and we used a similarly low detection significance threshold of $3.5 \sigma$. For each algorithm the source lists in the three bands were merged to create a single list input to EMLDETECT.

\subsection{Parameterization of detected sources}

Source candidates were parameterized in the three energy bands simultaneously via maximum likelihood fitting of the spatiallyvarying point spread function (PSF) using EMLDETECT. The free fit parameters were the source location, fixed to be the same in all energy bands, and the count rate in each energy band.

We retained detections with maximum likelihood $M L>8$ in at least one band. Extensive tests of simulated blank-field EPIC images have demonstrated that we expect approximately 1.5 false detections per full-band image due to background fluctuations alone.

Detections were overplotted on the image and examined by eye. Obvious false detections, e.g. in the wings of the point spread function of bright sources, were removed and the maximum likelihood fitting repeated.

This procedure was run using the EWAVELET candidate list and the PWXDETECT candidate list. The two source lists were merged, adding 0-5 extra sources per observation, and the maximum likelihood fitting repeated.

For each source, the parameters fitted by EMLDETECT were retained in each band where the source was detected with $M L>5$, else upper limits were calculated at the 95 per cent confidence level using the prescription of Kraft et al. (1991).

\subsection{Boresight correction}

2MASS counterpart positions were used to correct X-ray source positions for systematic shifts; this boresight correction was made iteratively. First, we cross-correlated the XEST catalog and the 2MASS All-Sky Catalogue of Point Sources (Cutri et al. 2003), using a $5^{\prime \prime}$ correlation radius and keeping only the minimum-distance match to compute the XEST-2MASS position offsets. The XEST positions were corrected by subtracting the median position offset. Then, we repeated this correction process, using a $3.5^{\prime \prime}$ correlation radius, until the median position offset became zero, which was obtained in a few steps. The boresight corrections are small $\left(0.4^{\prime \prime}-3.8^{\prime \prime}\right)$, and the final residual registration error between $2 \mathrm{MASS}$ and XEST sources is $0.9^{\prime \prime}-2.2^{\prime \prime}$.

The resultant boresight correction was applied to the X-ray source positions. The RMS error on the boresight shift of each field was calculated and added in quadrature to the statistical positional error calculated for each source in that field by EMLDETECT to give a $1 \sigma$ positional error circle.

We applied the same method to OM sources using $10^{\prime \prime}$ and $3^{\prime \prime}$ radii. We found larger boresight corrections $\left(1^{\prime \prime}-8^{\prime \prime}\right)$ but with residual registration errors lower than 1.6".

\subsection{Candidate TMC members}

To assess membership in TMC of the detected X-ray sources, we compiled a comprehensive membership catalog mostly based on the following references: Cohen \& Kuhi (1979), Kenyon \& Hartmann (1995), Briceño et al. (1998), White \& Ghez (2001), Briceño et al. (2002), Hartmann (2002), 
Hartigan \& Kenyon (2003), Luhman et al. (2003), Luhman (2004), Luhman (2006), White \& Hillenbrand (2004), Andrews \& Williams (2005), and Guieu et al. (2006). The catalog was subsequently used to decide which X-ray sources to use for the physical interpretation, and to assess which TMC members remained undetected. The adopted coordinates (mostly from 2MASS) are described in more detail below (Sect. 5.2.1).

\subsection{Extraction of source spectra and light curves}

Source events were extracted from circular regions centered on the EMLDETECT source positions. Although the off-axis PSF is obviously elongated, there is currently no available parameterization of an elliptical form of the PSF and the enclosed energy correction calculated by the SAS task ARFGEN in creating the ancillary response file is not energy dependent unless a circular source extraction region is used. We calculated the source extraction radius that maximized the source signal-to-noise ratio in the full energy band using: the total number of source counts derived by EMLDETECT for the full-band mosaicked EPIC image, the expected number of background counts per unit area in the source region, calculated from the full-band EPIC background map, and the circularly-symmetric EPIC PSF formulation of Ghizzardi et al. (2002). The SAS task REGION was used to calculate smaller extraction radii in cases where nearby contaminating sources existed. The source extraction radius was constrained to be between 8 " and $60^{\prime \prime}$.

Annular background extraction regions were calculated by the REGION task, with inner radii calculated such that the source count density (counts per sq. arcsec) had fallen to 30 per cent of the local background count density. The task excluded neighboring sources using the same criterion. The outer radius was simply 3 times the inner radius. For each case where a source of interest was clearly contaminated by counts from a neighboring source, the background region was individually defined to account for these, as an annulus around the contaminating source with inner and outer radii exactly enclosing the extraction region of the source of interest (with the source itself excluded with a larger circle), or as a number of circles within this annulus of identical size to the extraction region of the source of interest, chosen to avoid other nearby sources.

The same source and background regions were used for the extraction of PN and MOS products. Background products were scaled by the ratio of active detector area in the source region to that in the background region.

Light curves and spectra were extracted for all sources identified as members of Taurus-Auriga, for all other sources with more than 150 EPIC counts (see e.g. Scelsi et al. 2007), and all other sources detected by the Optical Monitor (Audard et al. 2007).

\subsubsection{Light curves}

Light curves were extracted for each instrument in the full-band only and used all available exposure time. Time intervals affected by high background were not excluded as intrinsic source variability can still be detected in these times. Also, the background flares provide a sensitive test of the reliability of the background subtraction.

The active exposure time in each bin was calculated, accounting for intervals when the instrument (or CCD) was not live, which are frequent when the instrument experiences high event rates during background flares. The background light curve was corrected for differences in active exposure

The time bin size was calculated such that the average number of EPIC source counts in each bin was 20. However, the minimum bin size was fixed to $100 \mathrm{~s}$ to produce easily-viewed plots and the maximum bin size was fixed to $1000 \mathrm{~s}$, the timescale on which flares are typically observed, to avoid short bright flares on faint sources being smoothed out. The same bin size was used for PN and MOS light curves to enable simple summation to form an EPIC light curve.

The light curves often have few counts per bin, in which case the traditional approach - calculating the source counts as $T-B$, where $T$ is the total number of counts in the source extraction region and $B$ is the number of expected background counts, with a symmetric $1 \sigma$ uncertainty calculated by propagating $\sqrt{(} N)$ errors - breaks down. We used the Bayesian approach of Kraft et al. (1991) to calculate the most-likely source count-rate and the 68 percent confidence interval in each bin with fewer than 20 net source counts or where the traditional $1 \sigma$ uncertainty extended below zero.

We also calculated a summed EPIC light curve. The counts in the PN, MOS1 and MOS2 source light curves were summed, as were those in the background light curves, and the net source light curve was calculated as described above. A PN-effective count rate was calculated for each bin by scaling the MOS active exposure time by the factor 0.370 (see above) and dividing the summed net counts by the sum of active exposure times in each bin. Two example light curves are shown in Fig. 3, one containing a strong flare (IQ Tau = XEST-14-006), and one containing some low-level activity (V807 Tau = XEST-04-012) seen in most stellar light curves in XEST.

\subsubsection{Spectra}

Source and background spectra were produced for each instrument using data in the GTIs used for source detection. This may be too conservative for some bright sources, but in trying to extract products automatically for sources with a wide range of count-rates, this is most straightforward. For sources whose light curves showed clear flares we also excluded times of flaring emission where possible, as we aimed to avoid measuring properties of a source in a temporary peculiar state.

PN spectra were extracted using events with patterns $0-4$, as the spectral response is not calibrated for higher patterns. Ancillary responses files (ARFs) were produced for each source spectrum using the SAS task ARFGEN. We used the canned response matrix files (RMFs) appropriate for SAS v6.1.0. For each source spectrum we used the PN RMF appropriate for patterns $0-4$, the observing mode and the source position on the CCD, and MOS RMFs appropriate for Imaging mode, patterns 0-12, and the orbit (date) of observation.

Three examples of EPIC PN spectra are shown in Fig. 4 together with the best DEM fits (see below).

\subsection{Overall sensitivity}

An example of a rich EPIC field in L1495, centered at V410 Tau, is displayed in Figure 5, showing a particularly deep observation within our survey. A typical exposure of $\approx 30 \mathrm{ks}$ duration with an average background contamination level reached a detection threshold of $\approx 9 \times 10^{27} \mathrm{erg} \mathrm{s}^{-1}$ on-axis and $\approx 1.3 \times 10^{28} \mathrm{erg} \mathrm{s}^{-1}$ at $10^{\prime}$ off-axis for an X-ray source with a thermal spectrum characteristic of $\mathrm{T}$ Tau stars (see below) subject to a hydrogen 

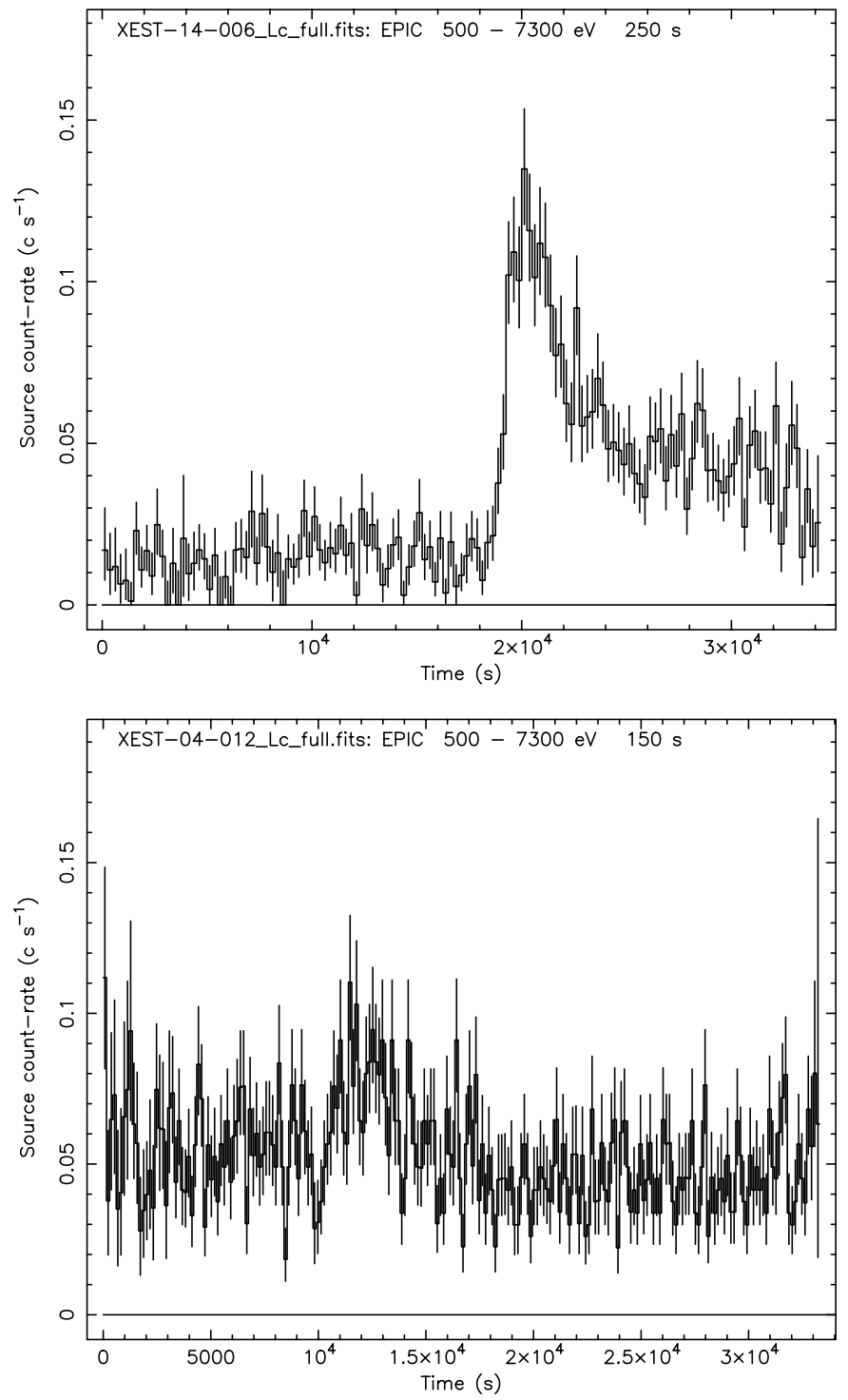

Fig. 3. Two X-ray light curves, summed from all three EPIC detectors in the energy range $0.5-7.3 \mathrm{keV}$. The total count rate has been re-normalized to the effective area of the PN. The upper panel shows IQ Tau = XEST-14-006 that flared strongly in the second half of the observation. The low-level episode used for spectral fitting was defined to cover the first $18 \mathrm{ks}$ (bin size: $250 \mathrm{~s}$ ). The lower panel shows V807 Tau = XEST-04-012, exhibiting low-level flaring variability seen in most XEST objects (bin size: $150 \mathrm{~s}$ ). Here, all data were used for the spectral fits.

absorption column density of $N_{\mathrm{H}}=3 \times 10^{21} \mathrm{~cm}^{-2}$. This threshold turns out to be appropriate to detect essentially every $\mathrm{T}$ Tau star in the surveyed fields. The precise detection limits depend on the level of particle background contamination (hence the exposure time obtained during low background radiation), and the off-axis angle. The effective area drops to about $50 \%$ at $10^{\prime}$ distance from the center and to $35 \%$ at the border of the detectors (15' distance).

A typical XEST exposure contained between 50 and $100 \mathrm{X}$-ray point sources (Table 3 ) of which, however, a minority (of order 10) are known TMC stellar members. The remainder are probably mostly extragalactic sources, but a non-negligible contribution may be defined by as yet unidentified new TMC members that may be embedded or be located behind the molecular cloud complex (see Scelsi et al. 2007 for a detailed study).
Hubble 4 = XEST-23-056, PN DEM fit
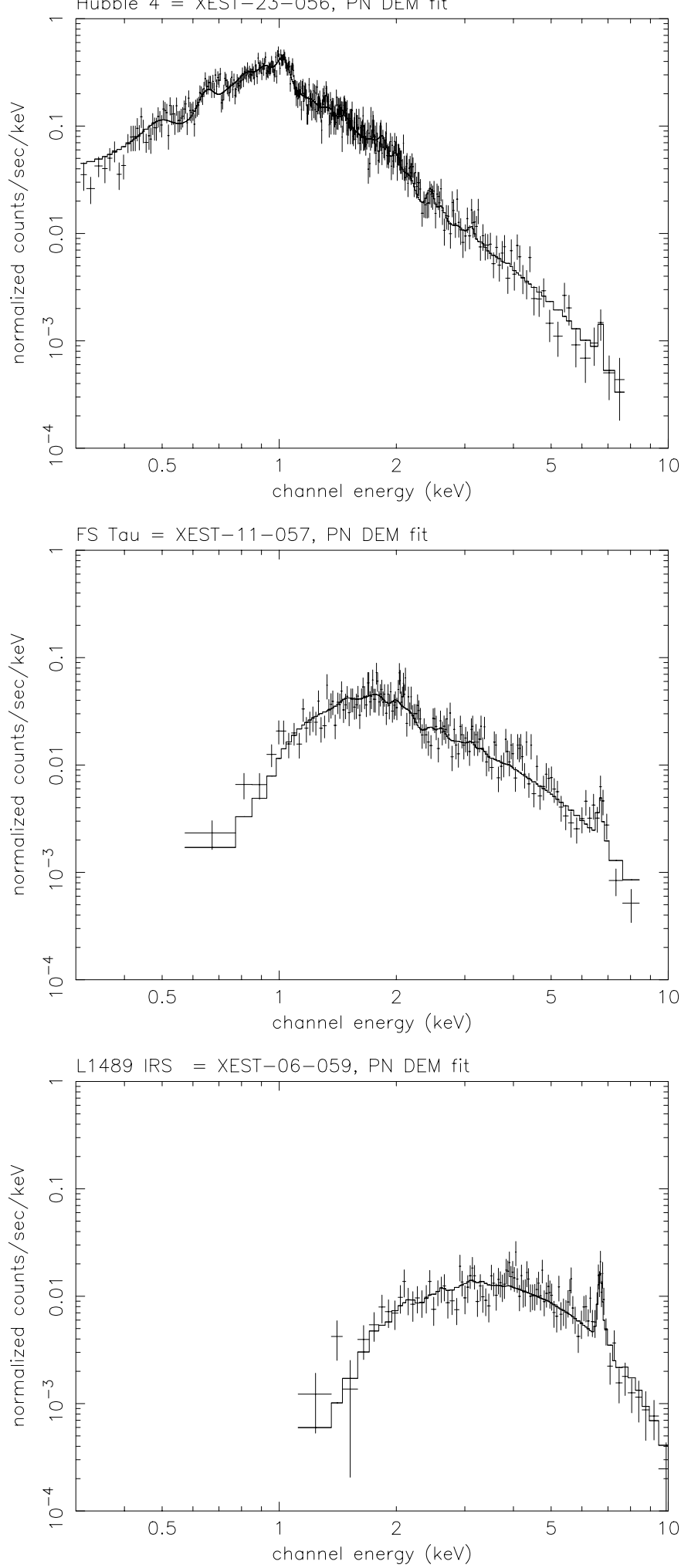

Fig. 4. Three example CCD spectra from the EPIC PN camera. All spectra extracted from the source position were binned to a minimum of 15 cts per bin. The histograms show the best fits derived from DEM fits. Top: Hubble 4 = XEST-23-056, an X-ray strong WTTS with modest absorption (total of $27648 \mathrm{cts}$ in EPIC, $N_{\mathrm{H}}=3.1 \times 10^{21} \mathrm{~cm}^{-3}$ ). Middle: FS Tau = XEST-11-057, an intermediately X-ray strong CTTS with strong absorption (total of $7398 \mathrm{cts}$ in EPIC, $N_{\mathrm{H}}=1.4 \times 10^{22} \mathrm{~cm}^{-3}$ ). Bottom: L1489 IRS = IRAS 04016+2610 = XEST-06-059, an X-ray bright protostar with very strong absorption (total of 3336 cts in EPIC, $\left.N_{\mathrm{H}}=6.6 \times 10^{22} \mathrm{~cm}^{-3}\right)$ 


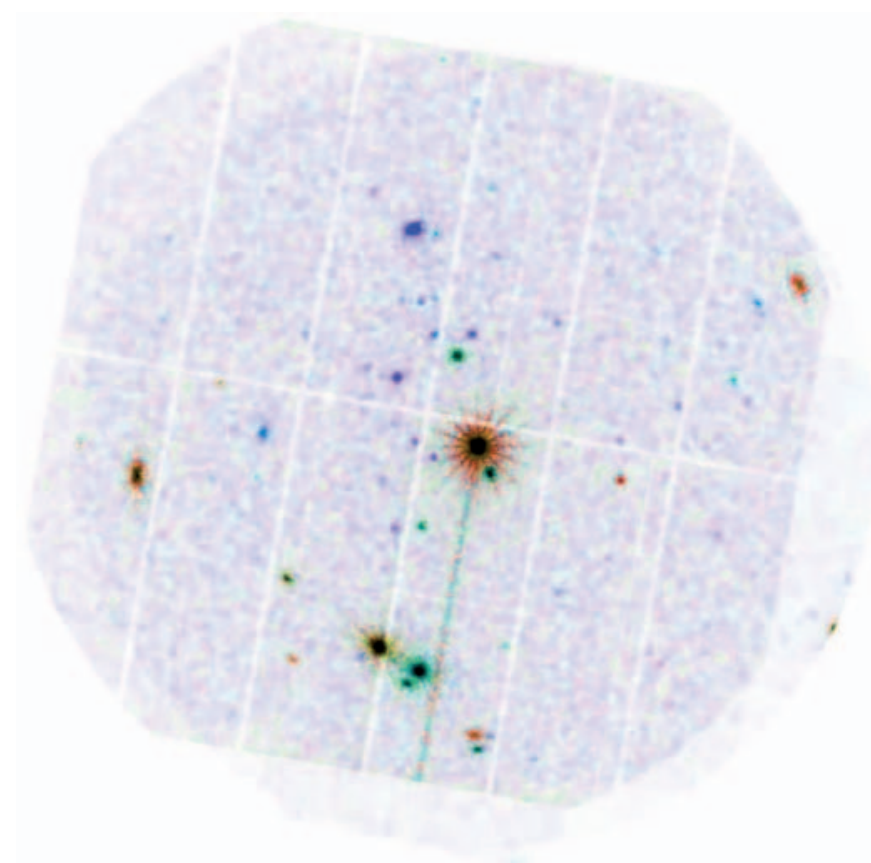

Fig. 5. Example of an XMM EPIC image (the field around V410 Tau = L1495). The field diameter is $30^{\prime}$, and the point-spread function has an $F W H M$ of about $5^{\prime \prime}$. North is up. The colors indicate X-ray hardness, the red-orange sources being softest and the blue sources being hardest. This exposure was longer than average ( $74 \mathrm{ks}$ compared to the more typical $30-40 \mathrm{ks}$ ). It contains $76 \mathrm{X}$-ray detections, of which 20 are TMC members.

\subsection{Chandra data}

The few exposures obtained with Chandra were reduced using standard strategies for ACIS, while we used the standard, reduced events file for the HRC-I observation around HR 1442. The ACIS-S observations of the fields around DG Tau and FS Tau used the "Very Faint Mode" of ACIS, while those around the fields of GV Tau, V410 Tau, L1527 used the "Faint Mode". The data were reduced in CIAO vers. 3.3.0.1 following the standard analysis threads ${ }^{6}$. These procedures included, for ACIS, corrections for charge transfer inefficiency and afterglow, and selection of good time intervals. Spectra were extracted from level 2 event files with DMEXTRACXT, and response and ancillary response files were generated with the MKRMF and MKARF tasks, respectively. We re-emphasize that these data are added here as they provide information on some objects undetected by XMMNewton, but the observations have been obtained with rather different exposure times, and the detectors in use vary (ACIS-S, ACIS-I, HRC-I). Some of the objects were at large off-axis angles and were thus subject to severe distortion (PSF FWHM up to several arcseconds). The data quality, e.g., positional accuracy or spectral information, is therefore very inhomogeneous for this sample.

\subsection{Spectral analysis}

\subsubsection{Multi-thermal fits}

For the spectral analysis, the MOS spectra provide little or no additional constraints for the many fainter sources, with a total count rate smaller than the count rate of the PN. To keep our analysis as uniform as possible, we therefore used only the PN

${ }^{6}$ http://asc.harvard.edu/ciao/threads/ spectrum for our spectral fits. Exceptions are those sources for which the PN data were not available, e.g., for sources whose image falls into a PN CCD gap, or for all sources in XEST exposure 26 for which the PN was not operational. To derive basic X-ray properties from the spectra, we followed two strategies. First, conventional one- or two-component spectral fits (1- $T$ or 2- $T$ henceforth) were performed. The two components define two plasmas with different temperatures $T_{1,2}$ and emission measures $\mathrm{EM}_{1,2}$. The latter parameters were fitted in XSPEC (Arnaud 1996) using the vapec thermal collisional ionization equilibrium model that includes emission lines and continua. The abundances were fixed at values typical for pre-main sequence or extremely active zero-age main-sequence stars (based on results from Telleschi et al. 2005; Argiroffi et al. 2004; Garcia-Alvarez et al. 2005; and Scelsi et al. 2005) ${ }^{7}$. The spectral components were subject to photoelectric absorption based on the Morrison \& McCammon (1983) cross sections. The equivalent hydrogen column density, $N_{\mathrm{H}}$, was treated as a further fit parameter. For the faintest sources, a 2- $T$ fit provided too many free parameters for the information contained in the spectrum. A 1- $T$ fit was performed in these cases.

\subsubsection{Emission-measure distributions}

For highly absorbed spectra, the multi-thermal fit method can become problematic because the softer component is strongly suppressed or may not be detected at all, biasing the fits toward high temperatures. This may further bias the total X-ray luminosity and $N_{\mathrm{H}}$. We therefore, in an alternative approach, combined several thermal components in such a way that they describe the shape of the differential emission measure distribution (DEM) in a similar way as previously found for nearby pre-main sequence stars or extremely active zero-age main sequence stars (Telleschi et al. 2005; Argiroffi et al. 2004; Garcia-Alvarez et al. 2005; Scelsi et al. 2005). In short, we have adopted a model in which the DEM shows one peak located at the temperature $T_{0}$, and two power-law distributions toward lower and higher temperatures, characterized by their power-law indices $\alpha$ and $\beta$, respectively. Given the poor temperature discrimination of CCD spectra in the softer X-ray range (in particular in the presence of significant photoelectric absorption), the power-law index on the low-temperature side was kept fixed at a value often found for magnetically active main-sequence and pre-main sequence stars, namely $\alpha=2$, while $\beta$ was treated as a free parameter. The DEM was cut off at $\log T=6.0$ below which EPIC is not sensitive to photons, and at $\log T=7.95$ above which EPIC is insensitive to temperature. Within this range, the DEM was binned to $\mathrm{d} \log T=0.1$, i.e., 20 bins (centered at $\log T=6.0$, $6.1, \ldots 7.9$ ) were used. The element abundances were fixed at the same values as those adopted for the 1- or 2- $T$ fits. Again, the absorbing hydrogen column density $N_{\mathrm{H}}$ was also fitted to the spectrum. The final fit parameter was the normalization, defined as the EM in the temperature bin at $T_{0}$. This method thus assumes that the DEM can be described by four fit parameters, namely $T_{0}, \beta, \mathrm{EM}_{0}$, and $N_{\mathrm{H}}$. We summed the EM over all EM bins used for the calculation to obtain the total $\mathrm{EM}_{t}$.

We set the following hard limits to the parameters: $-3 \leq$ $\beta \leq 1,2 \mathrm{MK} \leq T_{0} \leq 32 \mathrm{MK}$. In exceptional cases (very hard, absorbed spectra), we extended the range for $T_{0}$ to slightly

7 The adopted abundances are, with respect to the solar photospheric abundances given by Anders \& Grevesse (1989) $=$ AG89: $\mathrm{C}=0.45, \mathrm{~N}=$ $0.788, \mathrm{O}=0.426, \mathrm{Ne}=0.832, \mathrm{Mg}=0.263, \mathrm{Al}=0.5, \mathrm{Si}=0.309, \mathrm{~S}=$ $0.417, \mathrm{Ar}=0.55, \mathrm{Ca}=0.195, \mathrm{Fe}=0.195, \mathrm{Ni}=0.195$. 
higher values, although we emphasize that at such temperatures, the dominant contribution to the spectrum is a featureless bremsstrahlung continuum, apart from the Fe $\mathrm{K}$ complex at $6.7 \mathrm{keV}$.

For very faint sources, such fits provide too many degrees of freedom. We thus fixed one or more of the following parameters: $\beta$, usually fixed at a value of -1 which is often found for brighter sources, or $N_{\mathrm{H}}$, fixed at a value corresponding to the tabulated $A_{\mathrm{V}}$ assuming a standard gas-to-dust ratio (after Vuong et al. 2003), or both. In a few cases, it was necessary to fix $T_{0}$ as well, usually at $\log T_{0}=7$. Finally, for non-detections with reasonably wellknown $A_{\mathrm{V}}$, we derived upper limits to $L_{\mathrm{X}}$ as follows: We used the $95 \%$ confidence upper limits to the count rates derived at the optical or infrared position of the star, and then adopted an $N_{\mathrm{H}}$ derived from $A_{\mathrm{V}}$. We thus estimated the upper limit using an average thermal model. For brown dwarfs and very-low mass stars, we adopted $\beta=-3$ and $\log T_{0}=6.9$, characteristic values found for the detected low-mass objects. For more massive T Tau stars, we adopted $\beta=-1$ and $\log T_{0}=7$, again characteristic values found for the detected stars. These upper limits should only be used as rough estimates. For embedded sources, uncertain visual or infrared extinctions and the strong influence of $N_{\mathrm{H}}$ on the measured count rate make such estimates unreliable, which will therefore not be given. As we will predominantly use the DEM-fit results, we have not derived upper limits based on 1-T plasmas.

For both models, the total X-ray luminosity was computed for the energy range $0.3-10 \mathrm{keV}$ based on the integration of the best-fit model. We adopted a distance of $140 \mathrm{pc}$ for all objects.

The same spectral-fit procedures were applied to the Chandra ACIS data if feasible (most of the faint sources were interpreted only with a $1-T$ model). For the HRC data, we assumed a standard electron temperature of $10 \mathrm{MK}$ and estimated $N_{\mathrm{H}}$ from $A_{\mathrm{V}}$ using standard interstellar conversion ratios (Vuong et al. 2003 and references therein). The unabsorbed source flux and the corresponding luminosity were then estimated based on the PIMMS software ${ }^{8}$.

\section{Results}

Tables 4 - 11 summarize basic X-ray parameters and fundamental properties of all observed TMC members. We reproduce the first ten entries per table for illustration. The entire tables with comments and references are available in the electronic version of this paper.

All tables are of identical length, each line number referring to the same observation of the same target across all tables. The tables are sorted in increasing right ascension. The first column of each table gives the XEST catalog entry. This number is composed of the two-digit code corresponding to the exposure number listed in Table 1, followed by the X-ray source number for this exposure (including every X-ray source regardless of TMC membership). This source number is '000' for X-ray nondetections of known TMC members. The second column gives a conventional name frequently used in the literature. Preference was mostly given to variable-star names if available. Note that several objects were observed twice within the principal XEST project (in particular in exposures 23 and 24). They are listed as separate entries. XEST-25 is one of a series of exposures containing the TMC member AA Tau. This star is highly X-ray variable probably due to variable absorption by its disk. We use only one observation to report the typical X-ray properties of this star.

\footnotetext{
${ }^{8}$ http://heasarc.gsfc.nasa.gov/Tools/w3pimms.html
}

A more detailed analysis of the temporal behavior of AA Tau is given by Grosso et al. (in preparation). There are no other known TMC stellar members in this field.

The final portion of each table refers to results from the complementary Chandra observations. Here, we list TMC objects that were either not covered by XMM-Newton, regardless of their detection status with Chandra, or objects that were detected by Chandra but not by XMM-Newton. We also add FS Tau because XMM-Newton observed this star during a slow flux decrease, presumably from a flare, and Haro 6-5 B which is slightly contaminated by the wings of FS Tau $\mathrm{A}$ in the XMM-Newton observation. GV Tau is added because of the higher resolution of Chandra that attributes the flux to GV Tau A (Güdel et al. 2007b), while the embedded object Haro 6-10 B at 1.'3 remains undetected.

\subsection{The XEST $X$-ray results}

The individual X-ray results from our survey will be discussed in depth in the series of accompanying papers. We collect here the basic, statistical X-ray results for the stellar TMC sources. Our results refer to episodes during the observations that were not affected by outstanding stellar flares (i.e., flares in excess of the typical slow modulation within factors of $\approx 2$ of the average level), although low-level variability is common to premain sequence X-ray sources. Exceptions are CFHT-BD-Tau 1 $=$ XEST-17-068 that showed a flare with a slow decay, dominating most of the exposure time, and FS Tau = XEST-11-057, DH Tau $=$ XEST-15-040 and V830 Tau = XEST-04-016 that showed slowly decreasing light curves, probably following a strong flare starting before our exposures. Specific discussions of the time-dependent behavior of all observed sources will be given by Stelzer et al. (2007), Franciosini et al. (2007), Arzner et al. (2007a), and Audard et al. (2007).

Table 4 provides a summary of detection results for each XEST TMC member. The table lists the detection coordinates with statistical errors and the offsets from the expected position as given later in Table 7. For non-detections, the expected coordinates of the source is given (see below), which were used to calculate upper limits to the count rates. Then, the maximum likelihood (ML) is given for each detection, referring to the full (0.5-7.3 keV) band. Non-detections show ML $=0$. For the Chandra observations in Table 4, we list the "significance" of the detection as provided by the CIAO WAVDETECT algorithm instead of $M L_{\text {det }}$. Then, we list the total number of detected EPIC counts, the effective PN-equivalent on-axis exposure time that corrects for vignetting and also takes the (lower-sensitivity) MOS data into account, as described above. For Chandra, these numbers refer to the respective detector used for the observations. For XMM-Newton non-detections, the 95\% upper limits are reported. Next, the table gives the average count rate (onaxis equivalent rate for PN). Finally, we report a variability flag from inspection of the light curves; the codes have the following meaning: $0=$ no or only low-level variability, no flares visible that may dominate the X-ray spectrum; 1 = clear flaring observed and flare intervals removed in spectral fit; $2=$ clear flaring observed but flare intervals not removed; 3 = slow decay of flare throughout the observation, all data used. Examples for light curves with flag 1 (IQ Tau) and flag 0 (V807 Tau) are shown in Fig. 3. In the former case, spectral fits were obtained using only data during the low-level episode, defined by the first $18 \mathrm{ks}$ of the light curve. A more quantitative analysis of light curve variability and an interpretation of the role of flares in coronal heating will be given by Stelzer et al. (2007). 
Table 4. X-ray parameters of targets in XEST (1): Positions and count rates [first ten entries].

\begin{tabular}{|c|c|c|c|c|c|c|c|c|c|c|}
\hline XEST & Name & \begin{tabular}{r}
\multicolumn{2}{r}{$\mathrm{RA}_{X}$} \\
$\mathrm{~h} \mathrm{~m} \quad \mathrm{~s}$
\end{tabular} & $\begin{array}{r}\operatorname{Dec}_{X} \\
\operatorname{deg}{ }^{\prime} \prime \prime\end{array}$ & $\begin{array}{r}\text { Poserr } \\
\left({ }^{\prime \prime}\right)\end{array}$ & $\begin{array}{r}\text { Offset } \\
\left({ }^{\prime \prime}\right)\end{array}$ & $\mathrm{ML}_{\mathrm{det}}^{a}$ & Scts & $\begin{array}{r}T_{\exp } \\
(\mathrm{s})\end{array}$ & $\begin{array}{r}\text { Rate } \\
\left(\mathrm{ct} \mathrm{s}^{-1}\right)\end{array}$ & $\operatorname{Var}^{b}$ \\
\hline $27-115$ & HBC 352 & 35429.54 & 320302.2 & 1.04 & 0.89 & 9395 & 3832 & 15776 & 0.2429 & 0 \\
\hline $27-000$ & HBC 353 & 35430.17 & 320304.3 & 0.00 & 0.00 & 0 & $<183$ & 14469 & $<0.0127$ & 0 \\
\hline $06-005$ & HBC 358 AB & 40349.27 & 261053.1 & 1.48 & 1.22 & 1073 & 879 & 7361 & 0.1194 & 0 \\
\hline 06-007 & НBC 359 & 40350.82 & 261053.0 & 1.46 & 0.34 & 2921 & 1605 & 7664 & 0.2094 & 0 \\
\hline 06-059 & L1489 IRS & 40443.07 & 261856.3 & 1.46 & 0.10 & 7632 & 3336 & 46853 & 0.0712 & 0 \\
\hline $20-001$ & LkCa 1 & 41314.03 & 281909.9 & 1.58 & 1.71 & 455 & 336 & 7813 & 0.0430 & 0 \\
\hline $20-005$ & Anon 1 & 41327.28 & 281623.3 & 1.53 & 1.64 & 24658 & 8299 & 21634 & 0.3836 & 0 \\
\hline $20-000$ & IRAS $04108+2803 \mathrm{~A}$ & 41353.29 & 281123.4 & 0.00 & 0.00 & 0 & $<26$ & 29957 & $<0.0009$ & 0 \\
\hline $20-022$ & IRAS $04108+2803 \mathrm{~B}$ & 41354.72 & 281132.2 & 1.55 & 0.70 & 1145 & 696 & 30660 & 0.0227 & 1 \\
\hline $20-000$ & 2M J04141188+28 & 41411.88 & 281153.5 & 0.00 & 0.00 & 0 & $<54$ & 48645 & $<0.0011$ & 0 \\
\hline
\end{tabular}

${ }^{a}$ Maximum likelihood for detection for XMM-Newton data, CIAO WAVDETECT "Significance" for Chandra data.

${ }^{b}$ Variability flag: $0=$ no or only low-level variability; $1=$ clear flaring, flare intervals removed in spectral fit; $2=$ clear flaring observed but flare intervals not removed; 3 = slow decay of flare throughout observation, all data used.

Table 5. X-ray parameters of targets in XEST (2): Plasma parameters from the DEM fits [first ten entries].

\begin{tabular}{|c|c|c|c|c|c|c|c|c|c|c|}
\hline XEST & Name & $\begin{array}{l}N_{\mathrm{H}}(1 \sigma \text { range }) \\
\left(10^{22} \mathrm{~cm}^{-2}\right)\end{array}$ & $\begin{array}{l}T_{0} \quad(1 \sigma \text { range }) \\
(\mathrm{MK})\end{array}$ & ( $1 \sigma$ range $)$ & $\begin{array}{r}\mathrm{EM}_{t}^{a} \\
\left(10^{52}\right)\end{array}$ & $\begin{array}{l}L_{\mathrm{X}}^{b} \quad \text { (range) } \\
\left(10^{30} \mathrm{erg} \mathrm{s}^{-1}\right)\end{array}$ & $\begin{array}{r}\log \\
L_{\mathrm{X}} / L_{*}\end{array}$ & $\begin{array}{r}T_{\mathrm{av}} \\
(\mathrm{MK})\end{array}$ & $\chi_{\text {red }}^{2}$ & d.o.f. \\
\hline $27-115$ & HBC 352 & $0.22(0.19,0.25)$ & $\begin{array}{ll}6.5 & (5.0,8.2)\end{array}$ & $-0.60(-0.79,-0.47)$ & 25.21 & $2.657(2.43,2.87)$ & -3.03 & 12.00 & 0.85 & 129 \\
\hline $27-000$ & НBC 353 & 0.17 & 10.0 & -1.00 & & $<0.176$ & $<-4.04$ & $\ldots$ & $\ldots$ & $\ldots$ \\
\hline $06-005$ & HBC 358 AB & $0.01(0.00,0.04)$ & $4.7 \quad(3.0,7.5)$ & $-0.60(-1.24,-0.34)$ & 3.79 & $0.383(0.37,0.44)$ & -3.45 & 9.42 & 0.74 & 21 \\
\hline $06-007$ & HBC 359 & $0.01(0.00,0.02)$ & $7.2 \quad(5.8,8.9)$ & $-1.25(-1.84,-0.91)$ & 6.65 & $0.663(0.64,0.69)$ & -3.18 & 9.20 & 1.05 & 57 \\
\hline 06-059 & L1489 IRS & $6.63(6.18,7.09)$ & $50.9(30.9,60.5)$ & $-3.00(-3.00,0.02)$ & 30.39 & $4.471(4.07,4.91)$ & -3.63 & 40.42 & 0.94 & 99 \\
\hline $20-001$ & $\mathrm{LkCa} 1$ & $0.07(0.04,0.15)$ & $4.9 \quad(3.7,5.7)$ & $-3.00(-3.00,-1.94)$ & 2.66 & $0.232(0.20,0.37)$ & -3.80 & 4.35 & 0.57 & 18 \\
\hline $20-005$ & Anon 1 & $0.33(0.30,0.35)$ & $(6.4,9.1)$ & $-1.01(-1.26,-0.88)$ & 40.27 & $4.139(3.84,4.42)$ & -3.38 & 10.72 & 0.92 & 228 \\
\hline $20-000$ & IRAS $04108+2803 \mathrm{~A}$ & $\ldots$ & $\ldots$ & $\ldots$ & & $\ldots$ & 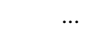 & $\ldots$ & $\ldots$ & $\ldots$ \\
\hline $20-022$ & IRAS $04108+2803 \mathrm{~B}$ & $5.68(2.99,8.19)$ & $(2.0,31.6)$ & -1.00 & 4.39 & $0.417(0.07,0.57)$ & -3.57 & 6.93 & 0.68 & 8 \\
\hline $20-000$ & $2 \mathrm{M} \mathrm{J} 04141188+28$ & 0.18 & 7.9 & -3.00 & $\ldots$ & $<0.028$ & $<-3.31$ & $\ldots$ & $\ldots$ & $\ldots$ \\
\hline
\end{tabular}

${ }^{a} \mathrm{EM}_{t}$ is sum of EM over all DEM bins from $\log T=6.0$ to $\log T=7.9$; given in units of $10^{52} \mathrm{~cm}^{-3}$.

${ }^{b} L_{\mathrm{X}}$ for $[0.3,10] \mathrm{keV}$, in units of $10^{30} \mathrm{erg} \mathrm{s}^{-1}$.

Table 6. X-ray parameters of targets in XEST (3): Plasma parameters from the 1-T and 2-T fits [first ten entries].

\begin{tabular}{lllrrrrrrrrr}
\hline \hline XEST & Name & $\begin{array}{l}N_{\mathrm{H}}(1 \sigma \text { range }) \\
\left(10^{22} \mathrm{~cm}^{-2}\right)\end{array}$ & $\begin{array}{r}T_{1}^{a} \\
(\mathrm{MK})\end{array}$ & $\begin{array}{r}T_{2} \\
(\mathrm{MK})\end{array}$ & $\begin{array}{r}\mathrm{EM}_{1}^{b} \\
\left(10^{52}\right)\end{array}$ & $\begin{array}{r}\mathrm{EM}_{2}^{b} \\
\left(10^{52}\right)\end{array}$ & $\begin{array}{r}L_{\mathrm{X}}^{c} \\
\left(10^{30}\right)\end{array}$ & $\begin{array}{r}\log \\
L_{\mathrm{X}} / L_{*}\end{array}$ & $\begin{array}{r}T_{\mathrm{av}} \\
(\mathrm{MK})\end{array}$ & $\begin{array}{c}\chi_{\text {red }}^{2 d} \\
\text { d.o.f. }\end{array}$ \\
\hline $27-115$ & HBC 352 & $0.19(0.17,0.21)$ & 7.54 & 23.77 & 10.35 & 11.52 & 2.307 & -3.09 & 13.81 & 0.87 & 128 \\
$27-000$ & HBC 353 & $\ldots$ & $\ldots$ & $\ldots$ & $\ldots$ & $\ldots$ & $\ldots$ & $\ldots$ & $\ldots$ & $\ldots$ & $\ldots$ \\
$06-005$ & HBC 358 AB & $0.00(0.00,0.03)$ & 4.29 & 14.26 & 1.41 & 2.12 & 0.346 & -3.49 & 8.82 & 0.73 & 20 \\
$06-007$ & HBC 359 & $0.00(0.00,0.02)$ & 5.33 & 15.19 & 3.06 & 3.53 & 0.628 & -3.20 & 9.34 & 1.09 & 56 \\
$06-059$ & L1489 IRS & $6.54(6.08,6.88)$ & $\ldots$ & 50.20 & $\ldots$ & 26.57 & 4.003 & -3.67 & 50.20 & 1.02 & 100 \\
$20-001$ & LkCa 1 & $0.07(0.00,0.21)$ & 1.39 & 8.35 & 1.76 & 0.82 & 0.205 & -3.85 & 2.46 & 1.27 & 15 \\
$20-005$ & Anon 1 & $0.28(0.27,0.29)$ & 8.58 & 24.12 & 19.05 & 14.11 & 3.473 & -3.46 & 13.32 & 1.02 & 228 \\
$20-000$ & IRAS 04108+2803 A & $\ldots$ & $\ldots$ & $\ldots$ & $\ldots$ & $\ldots$ & $\ldots$ & $\ldots$ & $\ldots$ & $\ldots$ & $\ldots$ \\
$20-022$ & IRAS 04108+2803 B & $7.86(3.40,28.0)$ & $\ldots$ & 11.59 & $\ldots$ & 5.95 & 0.588 & -3.42 & 11.59 & 0.61 & 8 \\
$20-000$ & 2M J04141188+28 & $\ldots$ & $\ldots$ & $\ldots$ & $\ldots$ & $\ldots$ & $\ldots$ & $\ldots$ & $\ldots$ & $\ldots$ & $\ldots$ \\
\hline
\end{tabular}

$a$ " $=$ " sign before number indicates that parameter was fixed. ${ }^{b}$ EM in units of $10^{52} \mathrm{~cm}^{-3} .{ }^{c} L_{\mathrm{X}}$ for $[0.3,10] \mathrm{keV}$, in units of $10^{30} \mathrm{erg} \mathrm{s}{ }^{-1} .{ }^{d} \mathrm{Numbers}$ followed by "C" denote C statistic (for low-background Chandra data).

Table 5 summarizes the basic spectral-fit results from our DEM method. The abundances were kept fixed at characteristic values as described in Sect. 4.12. The columns provide $N_{\mathrm{H}}$, the temperature of the DEM peak, $T_{0}$, the power-law index $\beta$ of the high-temperature DEM slope, the total EM integrated over all bins centered at values of $\log T=6.0$ to $\log T=7.9$ in steps of $d \log T=0.1$, and the inferred $L_{\mathrm{X}}$ in the $0.3-10 \mathrm{keV}$ range. We list $68 \%(1 \sigma)$ confidence ranges for $N_{\mathrm{H}}, \beta$, and $T_{0}$ in parentheses. For fixed parameters, no error range is given. For nondetections, the spectral parameters used to estimate upper limits to $L_{\mathrm{X}}$ are also given as fixed parameters. The next columns list the logarithms of the normalized luminosities $L_{\mathrm{X}} / L_{*}$ where the stellar bolometric luminosity $L_{*}$ is adopted from Table 9 , and the average electron temperature that is obtained as a mean of $\log T$ over the entire $\operatorname{DEM}$, where $\operatorname{DEM}(T)$ is used as a weight function, calculated analytically as follows:

$$
\begin{aligned}
& A=\left.\frac{T^{\alpha}}{\alpha T_{0}^{\alpha}}\left(\log T-\frac{1}{c \alpha}\right)\right|_{T_{l}} ^{T_{0}}+\left.\frac{T^{\beta}}{\beta T_{0}^{\beta}}\left(\log T-\frac{1}{c \beta}\right)\right|_{T_{0}} ^{T_{h}} \\
& B=\left.\frac{T^{\alpha}}{\alpha T_{0}^{\alpha}}\right|_{T_{l}} ^{T_{0}}+\left.\frac{T^{\beta}}{\beta T_{0}^{\beta}}\right|_{T_{0}} ^{T_{h}} \\
& \log T_{\mathrm{av}}=\frac{A}{B}
\end{aligned}
$$


Table 7. Fundamental parameters of targets in XEST (1): Names and coordinates [first ten entries].

\begin{tabular}{|c|c|c|c|c|c|c|}
\hline XEST & Name & $2 \mathrm{MASS}^{a}$ & IRAS $^{b}$ & Alternative names & $\begin{array}{l}\operatorname{RA}(\mathrm{J} 2000.0)^{c} \\
\mathrm{~h} \mathrm{~m} \mathrm{~s}\end{array}$ & $\begin{array}{l}\operatorname{Dec}(\mathrm{J} 2000.0)^{c} \\
\operatorname{deg},{ }^{c} \prime\end{array}$ \\
\hline $27-115$ & HBC 352 & $03542950+3203013$ & ... & NTTS 035120+3154SW & 35429.51 & 320301.4 \\
\hline $27-000$ & HBC 353 & $03543017+3203043$ & $\ldots$ & NTTS $035120+3154 \mathrm{NE}$ & 35430.17 & 320304.3 \\
\hline $06-005$ & HBC 358 AB & $04034930+2610520$ & $\ldots$ & NTTS 040047+2603W & 40349.31 & 261052.0 \\
\hline $06-007$ & HBC 359 & $04035084+2610531$ & & TTS $040047+2603$ & 40350.84 & 261053.2 \\
\hline $06-059$ & L1489 IRS & $04044307+2618563$ & $04016+2610$ & 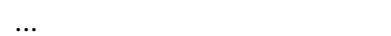 & 40443.07 & 261856.4 \\
\hline $20-001$ & $\mathrm{LkCa} 1$ & $04131414+2819108$ & $\ldots$ & HBC 365, V1095 Tau, JH 141 & 41314.14 & 281910.8 \\
\hline $20-005$ & Anon 1 & $04132722+2816247$ & & HBC 366, V1096 Tau & 41327.23 & 281624.8 \\
\hline $20-000$ & IRAS $04108+2803 \mathrm{~A}$ & $04135328+2811233$ & $04108+2803 \mathrm{~A}$ & L1495N IRS & 41353.29 & 281123.4 \\
\hline $20-022$ & IRAS $04108+2803$ B & $04135471+2811328$ & $04108+2803$ & $\ldots$ & 41354.72 & 281132.9 \\
\hline $20-000$ & 2M J04141188+28 & $04141188+2811535$ & $\ldots$ & $\ldots$ & 41411.88 & 281153.5 \\
\hline
\end{tabular}

${ }^{a}$ Nearest 2MASS entry within $5^{\prime \prime}$ to coordinates given by references $5,18,33$, or SIMBAD. Unlikely identifications in parentheses.

${ }^{b}$ Nearest IRAS catalog entry, within $10^{\prime \prime}$.

${ }^{c}$ 2MASS coordinates. For unlikely identifications, SIMBAD (S).

Table 8. Fundamental parameters of targets in XEST (2): Multiplicity [first ten entries].

\begin{tabular}{|c|c|c|c|c|}
\hline XEST & Name & Comp & $\begin{array}{r}\text { Separations } \\
\left({ }^{\prime \prime}\right)\end{array}$ & Refs. \\
\hline $27-115$ & HBC 352 & 1 & $\ldots$ & $\ldots$ \\
\hline $27-000$ & HBC 353 & 1 & $\ldots$ & $\ldots$ \\
\hline 06-005 & HBC 358 AB & 3 & $0.15,1.55$ & $11,20,31,40$ \\
\hline 06-007 & HBC 359 & 1 & $\ldots$ & $\ldots$ \\
\hline 06-059 & L1489 IRS & 1 & $\ldots$ & $\ldots$ \\
\hline 20-001 & LkCa 1 & 1 & $\ldots$ & $\ldots$ \\
\hline $20-005$ & Anon 1 & 1 & $\ldots$ & $\ldots$ \\
\hline $20-000$ & IRAS $04108+2803 \mathrm{~A}$ & 1 & $\ldots$ & $\ldots$ \\
\hline $20-022$ & IRAS $04108+2803 \mathrm{~B}$ & 1 & $\ldots$ & $\ldots$ \\
\hline $20-000$ & 2M J04141188+28 & 1 & $\ldots$ & $\ldots$ \\
\hline
\end{tabular}

Table 9. Fundamental parameters of targets in XEST (3): Photometry and spectroscopy [first ten entries].

\begin{tabular}{llrrrrrrr}
\hline \hline XEST & Name & Spec $^{a}$ & Refs. & $\begin{array}{r}A_{\mathrm{V}}^{a} \\
(\mathrm{mag})\end{array}$ & $\begin{array}{r}A_{J} \\
(\mathrm{mag})\end{array}$ & $\begin{array}{r}T_{\text {eff }}^{a} \\
(\mathrm{~K})\end{array}$ & $\begin{array}{r}L_{*}^{b} \\
\left(L_{\odot}\right)\end{array}$ & Refs. \\
\hline $27-115$ & HBC 352 & G0 & 27 & 0.87 & 0.25 & 6030 & 0.740 & 27 \\
$27-000$ & HBC 353 & G5 & 27 & 0.97 & 0.28 & 5770 & 0.500 & 27 \\
$06-005$ & HBC 358 AB & M2 & 27 & 0.21 & 0.06 & 3580 & 0.280 & 27 \\
$06-007$ & HBC 359 & M2 & 27 & 0.49 & 0.14 & 3580 & 0.260 & 27 \\
$06-059$ & L1489 IRS & K4 & 59 & 10.20 & $\ldots$ & 4500 & 4.900 & 10,59 \\
$20-001$ & LkCa 1 & M4 & 5 & 0.00 & 0.00 & 3270 & 0.380 & 5,27 \\
$20-005$ & Anon 1 & M0 & 5 & 1.32 & 1.03 & 3850 & 2.600 & 5,27 \\
$20-000$ & IRAS 04108+2803 A & $\ldots$ & $\ldots$ & $\ldots$ & $\ldots$ & $\ldots$ & $\ldots$ & $\ldots$ \\
$20-022$ & IRAS 04108+2803 B & $\ldots$ & $\ldots$ & $\ldots$ & $\ldots$ & 3500 & 0.400 & 10 \\
$20-000$ & 2M J04141188+28 & M6.25 & 33 & $\ldots$ & 0.28 & 2962 & 0.015 & 33 \\
\hline
\end{tabular}

${ }^{a}$ For multiples, first number or spectral type refers to primary, second to secondary component.

${ }^{b}$ For multiples, three numbers give primary/secondary/total system luminosity.

${ }^{c}$ Referring to $L_{\mathrm{bol}}$ as derived from integration of the optical and infrared spectrum.

where $c=\ln 10 \approx 2.3$ (note that we use decadic logarithms throughout), and we define the limits of the integration as $T_{l}=$ $10^{6} \mathrm{~K}$ and $T_{h}=10^{8} \mathrm{~K}$. Finally, we list the reduced $\chi_{\text {red }}^{2}$ achieved by the fit together with the number of degrees of freedom (d.o.f.).

Table 6 equivalently reports the results from 2- $T$ or, for faint sources, 1- $T$ fits. The columns give $N_{\mathrm{H}}$ (with errors if fitted), $T_{1}, T_{2}$, and the corresponding $\mathrm{EM}_{1}, \mathrm{EM}_{2}$. If only one component was fitted, its temperature and EM values are listed in the columns for $T_{2}$ and $\mathrm{EM}_{2}$ if the temperature exceeds $10 \mathrm{MK}$, and in the columns for $T_{1}$ and $\mathrm{EM}_{1}$ otherwise. If a temperature was held fixed, its value is preceded by a "=" sign. Further, the table gives $L_{\mathrm{X}}, \log \left(L_{\mathrm{X}} / L_{*}\right)$, and $T_{\mathrm{av}}$, the latter defined as the
EM-weighted logarithmic average of the temperature in the case of two non-vanishing components:

$\log T_{\mathrm{av}}=\frac{\mathrm{EM}_{1} \log T_{1}+\mathrm{EM}_{2} \log T_{2}}{\mathrm{EM}_{1}+\mathrm{EM}_{2}}$.

Finally, the table lists the $\chi_{\text {red }}^{2}$ values and the number of degrees of freedom (d.o.f.).

Errors of EM and $L_{X}$ are difficult to compute, and their meaning is not unequivocal. Our EM models are rather crude approximations to true emission measure distributions. Formal error ranges for the EM values may therefore bear little relevance. Further, $L_{\mathrm{X}}$ is a complicated function of the thermal emission model. Although resolved spectral features can usually be fitted 
Table 10. Fundamental parameters of targets in XEST (4): Age, mass, radius, rotation [first ten entries].

\begin{tabular}{llrlrlrrrr}
\hline \hline XEST & Name & $\begin{array}{r}\text { Age }^{a, b} \\
(\mathrm{Myr})\end{array}$ & $\begin{array}{l}\text { Mass }^{a, c} \\
\left(M_{\odot}\right)\end{array}$ & Refs. & $\begin{array}{l}\text { Radius }^{d} \\
\left(R_{\odot}\right)\end{array}$ & $\begin{array}{r}P \\
(\mathrm{~d})\end{array}$ & Refs. & $\begin{array}{r}v \sin i \\
\left(\mathrm{~km} \mathrm{~s}^{-1}\right)\end{array}$ & Refs. \\
\hline $27-115$ & HBC 352 & $\ldots$ & 1.05 & 2 & 0.79 & $<0.53$ & $\mathrm{C}$ & $>75.00$ & 48 \\
$27-000$ & HBC 353 & $\ldots$ & $\ldots$ & $\ldots$ & 0.71 & $<4.08$ & $\mathrm{C}$ & 8.80 & 2 \\
$06-005$ & HBC 358 AB & 3.26 & 0.41 & 27 & 1.38 & $\ldots$ & $\mathrm{C}$ & $<10.00$ & 2 \\
$06-007$ & HBC 359 & 3.54 & 0.41 & 27 & 1.33 & $\ldots$ & $\mathrm{C}$ & $<10.00$ & 48 \\
$06-059$ & L1489 IRS & 0.80 & 1.45 & 10 & 3.65 & $<4.02$ & $\mathrm{C}$ & 46.00 & 10 \\
$20-001$ & LkCa 1 & 0.87 & 0.27 & 5 & 1.93 & $<4.18$ & $\mathrm{C}$ & 23.30 & 48 \\
$20-005$ & Anon 1 & 0.50 & 0.56 & 5 & 3.63 & $\ldots$ & $\ldots$ & $\ldots$ & $\ldots$ \\
$20-000$ & IRAS 04108+2803 A & $\ldots$ & $\ldots$ & $\ldots$ & $\ldots$ & $\ldots$ & $\ldots$ & $\ldots$ & $\ldots$ \\
$20-022$ & IRAS 04108+2803 B & 1.60 & 0.36 & 10 & 1.72 & $<6.23$ & $\mathrm{C}$ & 14.00 & 10 \\
$20-000$ & 2M J04141188+28 & $\ldots$ & 0.08 & 46 & 0.47 & $\ldots$ & $\ldots$ & $\ldots$ & $\ldots$ \\
\hline
\end{tabular}

${ }^{a}$ For binaries, first number refers to primary, second to secondary component (calculated from $L_{*}$ and $T_{\text {eff }}$ ).

${ }^{b}$ Ages derived after Siess et al. (2000) using the same principal parameters as for masses, quoted in Table 9.

${ }^{c}$ Masses derived after Siess et al. (2000) using principal parameters quoted in Table 9.

${ }^{d}$ For multiples, radius is given only for primary if luminosity of primary is explicitly known

Table 11. Fundamental parameters of targets in XEST (5): Accretion and evolution [first ten entries].

\begin{tabular}{|c|c|c|c|c|c|c|c|c|c|}
\hline XEST & Name & $\begin{array}{r}\dot{M}(\min / \max )^{a} \\
\left(M_{\odot} \mathrm{yr}^{-1}\right)\end{array}$ & Refs. & $\begin{array}{r}E W(\mathrm{H} \alpha)^{b} \\
(\AA)\end{array}$ & $\begin{array}{l}\text { TTS } \\
\text { type }\end{array}$ & Refs. $^{c}$ & $\begin{array}{r}\mathrm{IR}^{d} \\
\text { class }\end{array}$ & Refs. $^{d}$ & Type \\
\hline $27-115$ & HBC 352 & $\ldots$ & $\ldots$ & 0 & $\mathrm{~W}$ & 29 & III & 1,27 & 3 \\
\hline $27-000$ & HBC 353 & $\ldots$ & $\ldots$ & 0 & W & 29 & III & 27 & 3 \\
\hline $06-005$ & HBC 358 AB & $<8.97$ & 20 & 4- 10 & $\mathrm{~W} / \mathrm{W}$ & 37,29 & III & 1,27 & 3 \\
\hline 06-007 & HBC 359 & $\ldots$ & $\ldots$ & $2-9$ & W & 56,37 & III & 27 & 3 \\
\hline $06-059$ & L1489 IRS & -7.15 & 59 & $41-56$ & $\mathrm{C}^{*}$ & 59,29 & I & $1,59,27$ & 1 \\
\hline $20-001$ & LkCa 1 & $<-9.72$ & 58 & 3- 4 & W & 29,45 & III & 1,27 & 3 \\
\hline $20-005$ & Anon 1 & $<-8.94$ & 58 & $1-3$ & W & 37,29 & III & 1,27 & 3 \\
\hline $20-000$ & IRAS $04108+2803 \mathrm{~A}$ & $\ldots$ & $\ldots$ & 37 & $\mathrm{C}$ & 29 & II & 27 & 2 \\
\hline $20-022$ & IRAS $04108+2803 \mathrm{~B}$ & $\ldots$ & $\ldots$ & $\ldots$ & $\ldots$ & $\ldots$ & I & 59,27 & 1 \\
\hline $20-000$ & 2M J04141188+28 & -10.00 & 46 & 250 & $\mathrm{C}$ & 46 & ... & $\ldots$ & 4 \\
\hline
\end{tabular}

${ }^{a}$ Range of $\dot{M}$ reported in literature given. For multiple systems, numbers refer to primary or integrated system.

${ }^{b}$ Range of $E W$ reported in literature given. For multiple systems, numbers refer to primary or integrated system.

${ }^{c}$ For $E W$ range, first reference for minimum, second for maximum reported.

${ }^{d}$ Infrared classification; double entries: '/' for transition objects, '+' for components, ';' for different types, ', for different references. FS = flat-spectrum source.

sufficiently well in a $\chi^{2}$ sense using a number of thermal components that describe the absorbed, observed spectral flux density, the major uncertainty in $L_{\mathrm{X}}$ is introduced by fitting (and correcting for) $N_{\mathrm{H}}$. An instability discussed below makes determinations of $L_{\mathrm{X}}$ rather uncertain once cool plasma components are subject to large absorption while the spectrum is of modest quality. To quantify this latter effect that dominates the uncertainty of $L_{X}$ in many cases, we proceeded as follows. We adopted the upper and lower $1 \sigma$ bounds of $N_{\mathrm{H}}$ (if $N_{\mathrm{H}}$ was a fit parameter), kept these values fixed and refitted the spectrum. The $L_{\mathrm{X}}$ values thus determined, $L_{\mathrm{X}, 1}$ and $L_{\mathrm{X}, 2}$ for the lower and the upper bound, are taken to bracket the error range of the tabulated $L_{X}$. We performed this error analysis for the DEM fit method. We graphically show the magnitude of the ratio $L_{\mathrm{X}, 2} / L_{\mathrm{X}, 1}$ in Fig. 6 as a function of the best-fit $N_{\mathrm{H}}$ (set to $0.01 \times 10^{22} \mathrm{~cm}^{-2}$ for sources with $N_{\mathrm{H}}$ smaller than this value) and the number of counts used in the spectral fit. Expectedly, the error range is largest for faint and strongly absorbed sources, and smallest for bright and weakly absorbed sources. For 83 sources, $L_{\mathrm{X}, 2} / L_{\mathrm{X}, 1} \leq 2$ and for 48 sources, $L_{\mathrm{X}, 2} / L_{\mathrm{X}, 1}>2$. For 99 sources, $L_{\mathrm{X}, 2} / L_{\mathrm{X}, 1} \leq 3$ and for 32 sources, $L_{\mathrm{X}, 2} / L_{\mathrm{X}, 1}>3$. Gagné et al. (2004) described a similar analysis performed for errors in $L_{\mathrm{X}}$ for Chandra observations of the $\rho$ Ophiuchus Cloud, with similar conclusions.
We note, however, that $\mathrm{T}$ Tau stars are manifestly variable on timescales of hours, typically within a factor of at least two, while most of the EM errors are formally much smaller. A detailed variability analysis of all XEST sources will be presented by Stelzer et al. (2007). Therefore, for the majority of our sources, the formal uncertainties from the spectral fits underestimate the true uncertainty. Realistically, thus, standard error bars of (at least) 0.3 dex should be adopted for all values of $L_{\mathrm{X}}$ (and EM). Larger statistical errors from spectral fits are typically found for $N_{\mathrm{H}} \gtrsim 2 \times 10^{21} \mathrm{~cm}^{-2}$ and $\lesssim 600 \mathrm{cts}$ in the spectrum, or in regions where cts $/\left(N_{\mathrm{H}} / 10^{22} \mathrm{~cm}^{-2}\right) \lesssim 500$.

\subsection{The XEST catalog}

Tables 7-11 identify the X-ray sources and provide a summary of the fundamental properties of the stellar systems. The parameters were extracted from the existing literature, in particular from Cohen \& Kuhi (1979), Kenyon \& Hartmann (1995), Briceño et al. (1998), White \& Ghez (2001), Briceño et al. (2002), Hartmann (2002), Hartigan \& Kenyon (2003), Luhman et al. (2003), Luhman (2004), White \& Hillenbrand (2004), Andrews \& Williams (2005), and Guieu et al. (2006). Several further references are listed in the bibliography summary in the table 


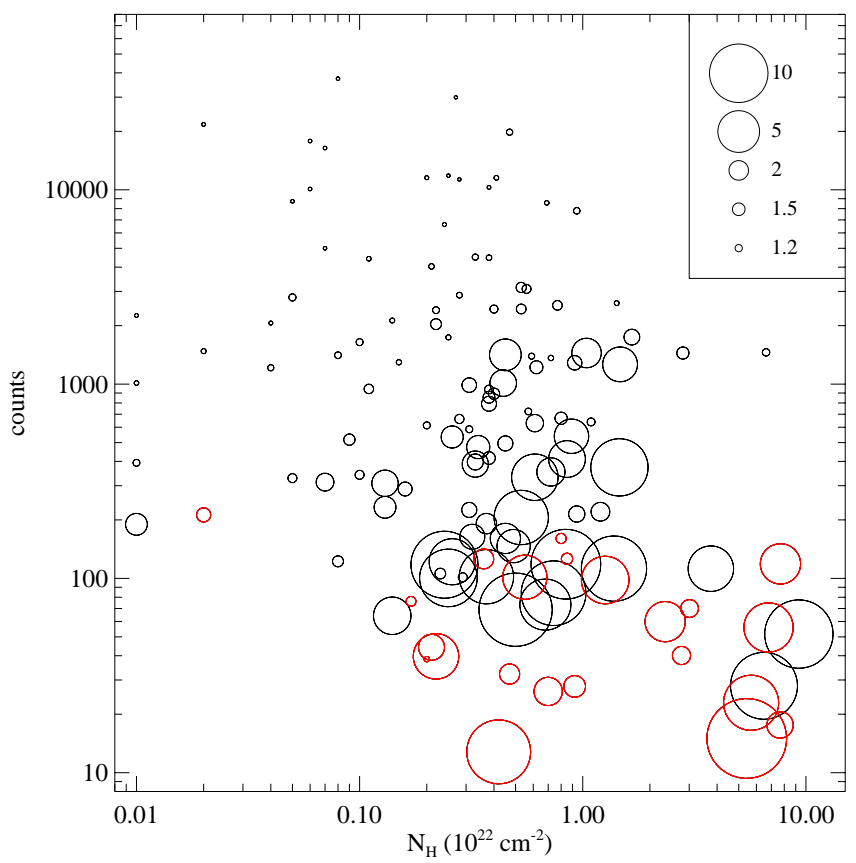

Fig. 6. Magnitude of the error ranges for $L_{X}$, estimated from maximum variations of $N_{\mathrm{H}}$ (see text for details), as a function of the best-fit $N_{\mathrm{H}}$ and the number of counts used in the spectral fit. Results from the DEM fit method are shown. The circle size scales with the logarithm of the ratio between the upper and the lower bounds of $L_{\mathrm{X}}$. Red circles denote sources for which the $\beta$ parameter was held fixed.

section. For the suspected late-B member of TMC, HD 28867 , all basic information except for the 2MASS identification comes from Walter et al. (2003) and references therein. A short description of the table entries follows.

\subsubsection{Names, coordinates, and multiplicity}

Table 7 lists the 2MASS catalog entry likely to be identified with the X-ray source. For this identification, we first adopted object coordinates mostly from Briceño et al. (2002), Luhman et al. (2003), Luhman (2004), and Guieu et al. (2006) if available, or from SIMBAD in other cases, and then identified the closest object in the most recent 2MASS catalog version (Cutri et al. 2003) within a circle of radius $5^{\prime \prime}$ around this position. In three cases, no 2 MASS object was found within $5^{\prime \prime}$. The next columns of Table 7 give the nearest IRAS catalog entry, confined to a search radius of $10^{\prime \prime}$ or directly identified from SIMBAD, and a selection of commonly used alternative names. Finally, the J2000.0 coordinates refer to the adopted 2MASS identifications. If no 2MASS identification is given, the coordinates were taken from SIMBAD, marked with '(S)' after the coordinates.

Table 8 provides binary information, i.e., the number of components, the approximate component separations, and references. Note that binary components with a separation of $\lesssim 5^{\prime \prime}$ are unresolved in XMM-Newton observations, and pairs at 5$10^{\prime \prime}$ are difficult to separate. Typically, binaries with separations up to about $10^{\prime \prime}$ have been recorded as a single X-ray source (e.g., V819 Tau, but note the case of HP Tau/G2 and G3, two stars with a separation of $10^{\prime \prime}$ for which a joint spectral model was derived, while the count rates and thus the luminosities were estimated separately based on PSF fitting).

\subsubsection{Photometry and spectroscopy}

Table 9 lists spectral types, visual and $J$-band extinctions ( $A_{\mathrm{V}}$ and $A_{J}$, respectively, the latter only for the primary in multiple systems), effective temperatures ( $T_{\text {eff }}$; for binaries, separately given for primary and secondary if available), and bolometric luminosities $\left(L_{*}\right)$ of the stars. The latter are bolometric luminosities of the stellar photospheres derived from the optical or near-IR except in cases where only the IR bolometric luminosity, possibly including contributions from the disk and/or the envelope, was available (these cases are marked with " $\mathrm{Lb}$ " in the reference column). In the case of binaries, $L_{*}$ is given for the primary and the secondary if available, and the entire system. The component and the system values may originate from different references, which accounts for some discrepancies with regard to the sum of the component $L_{*}$. Because spectral types, $A_{\mathrm{V}}, A_{J}$, and $L_{*}$ sensitively depend on the interpretation of the measured $T_{\text {eff }}$ and the optical and near-infrared photometry, we attempted to use the same references for these parameters for a given star, and opted predominantly for Briceño et al. (2002), Luhman et al. (2003) and Luhman (2004), and Kenyon \& Hartmann (1995) (in this sequence) for single stars or integrated multiple systems, and for White \& Ghez (2001) and Hartigan \& Kenyon (2003) for binary components. Significant information for deeply embedded sources was adopted from White \& Hillenbrand (2004), and for brown dwarfs from Guieu et al. (2006), Luhman et al. (2003), and Luhman (2004). Grosso et al. (2007a) rederived $T$ eff of some BDs from the spectral types, while we report here values as originally published. Strict consistency was not possible, in particular for $A_{\mathrm{V}}$ and $A_{J}$ that had to be adopted from different authors and that are inconsistent in several cases (the second references in the last column mostly refer to $A_{\mathrm{V}}$ ).

\subsubsection{Ages, masses, and rotation}

Table 10 lists ages and masses, $M$, that we derived from $T_{\text {eff }}$ and $L_{*}$ as listed in Table 9 using the Siess et al. (2000) isochrones. For binaries, component ages and masses are given if the fundamental parameters were available separately. In a few cases, we did not find a solution from the Siess et al. (2000) tracks; if available, some masses (and ages) were therefore taken directly from the literature (see footnotes to Table 10). The references specifically used for $T_{\text {eff }}$ and $L_{*}$ are listed. Assessing the uncertainties of these parameters is difficult because the uncertainties in $T_{\text {eff }}$ and $L_{*}$ are usually not reported in the literature. If multiple measurements have been reported for an object, then the agreement was (for the TMC) within $100 \mathrm{~K}$ for $T_{\text {eff }}$ for about half of the objects, and within $150 \mathrm{~K}$ for two thirds. We conservatively adopt an error of $\pm 150 \mathrm{~K}$ for $T_{\text {eff }}$. Using the Siess et al. calculations for the regions of densest population in the HRD $\left(T_{\text {eff }}=4700,4000\right.$, and $3500 \mathrm{~K}$ with $L_{*}=2,1$, and $0.1 L_{\odot}$, respectively), we find mass uncertainties of $\pm(0.07-0.16) M_{\odot}$ or a fractional mass uncertainty of about $\pm(10-20) \%$ (higher for cooler stars). An uncertainty in $T_{\text {eff }}$ also affects the age estimate, resulting in typical deviations of $\pm(0.1-0.18)$ dex (factors of 1.3-1.5) from the best-fit value. The age estimate is further affected by uncertainties in $L_{*}$ (while $L_{*}$ has little influence on the mass estimate in the region where the evolutionary tracks are nearly vertical). We found that in the region of $T_{\mathrm{eff}} \approx 3500-4000 \mathrm{~K}$, a shift of $\mathrm{d} \log L_{*}$ in $\log L_{*}$ results in a shift of approximately $-\mathrm{d} \log L_{*}$ in age. For $\approx 80 \%$ of the TMC objects with multiple reports of $L_{*}$ in the literature, deviations are up to a factor of 2 ( $0.3 \mathrm{dex})$, but often much smaller. We thus adopt a conservative, characteristic uncertainty of factors of 2.6-3.0 for the ages, noting that this 
is a gross overestimate for many objects, while for a minority of objects, in particular for embedded protostars with poor $L_{*}$ determinations, ages may become entirely unreliable.

The radius, $R$, was calculated from $L_{*}=4 \pi R^{2} \sigma T_{\mathrm{eff}}^{4}$, where

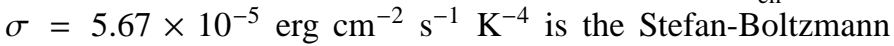
constant. Rotation periods $P$ are given as measured by various authors. The values were taken from Rebull et al. (2004) who give a comprehensive list of references (not reproduced here). That work includes periods of the lower-mass sample. We have added further rotation periods (Rebull, private communication) extracted from the same bibliography. If not available from the literature, rotation periods were estimated as upper limits if spectroscopic $v \sin i$ measurements were available (listed in Col. 9, with references in column 10, many again referring to the tabulations and the bibliography given by Rebull et al. 2004). In those cases, $P \leq 2 \pi R /(v \sin i)$, and the reference column for these calculated periods contains a "C".

\subsubsection{Accretion and evolutionary stage}

Table 11 provides parameters relevant for the interpretation of the evolutionary status. Column 3 lists mass accretion rates $\dot{M}$. Accretion rates may be variable, and different indirect methods have been used by previous studies to estimate $\dot{M}$, hence significantly discrepant values may be found in the published literature. In those cases, we provide ranges, giving a reference for each the minimum and maximum values reported. The same applies to the equivalent width of the $\mathrm{H} \alpha$ line $[E W(\mathrm{H} \alpha)]$, reported in Col. 5. In multiple systems, both of the above values refer to the primary star even if, in a few cases, a separate measurement was available for a companion. We adopted this strategy because the primary star is most likely also to be the dominant X-ray source.

We assessed the "T Tauri" type (classical or weak-line) based on $E W$ as follows. For spectral types $\mathrm{G}$ and $\mathrm{K}$, stars with $E W(\mathrm{H} \alpha) \geq 5 \AA$ are defined as classical T Tauri stars (C), all other stars are weak-line T Tauri stars (W). For early-M stars, the separation was adopted at $E W(\mathrm{H} \alpha)=10 \AA$, and for mid-M type stars, we adopted $E W(\mathrm{H} \alpha)=20 \AA$ for discrimination. For late-M stars, i.e., typically BDs, the low continuum makes a sensible definition difficult. We will therefore not include BDs when studying differences between CTTS and WTTS, although we have adopted the criterion described by Barrado y Navascués \& Martín (2003) that defines accreting stars and BDs as those that show $L(\mathrm{H} \alpha) / L_{*}$ greater than the chromospheric saturation limit. This criterion also supports our classifications at earlier spectral types. Borderline cases are marked with a question mark. In such cases, we also included other supporting evidence. For example, KPNO-Tau 14 (XEST-18-004) was classified as "W?" (and subsequently as "type 3", see below) despite one published measurement of $E W(\mathrm{H} \alpha)$ somewhat higher than the saturation limit, because there is one report with much lower $E W(\mathrm{H} \alpha)$, a very low upper limit to $\dot{M}$, and no $K$ band excess (Luhman et al. 2003; Muzerolle et al. 2005). "C*" denotes sources for which accretion signatures are evident, but that are embedded and share characteristics with protostars. We have added some "C' and "W" designations from Luhman (2004) even if the explicit $E W$ values were not given.

Column 8 lists the "Young Stellar Object" (YSO) infrared class as derived from the infrared spectral energy distribution, which is thought to provide information on the presence of disks and molecular envelopes. Class 0 and I objects are protostars, Class II objects are disk-surrounded T Tau stars, whereas disk signatures are weak or absent in Class III stars. "FS" designates "flat spectrum" sources, intermediate between Class I and II. Most entries refer to IRAS measurements (and were mostly adopted from Kenyon \& Hartmann 1995), but some recent information from Spitzer is also available (Hartmann et al. 2005). If discrepant entries are given, they are separated by a semicolon, and so are the corresponding references in the subsequent column. References referring to the same classification are separated by a comma. Transition objects are designated by two types separated by a "/", while for multiple systems with different component classifications, we give the two classes separated by "+".

There is a rather good correlation between TTS type indicating presence or absence of accretion (CTTS and WTTS) and YSO infrared class (Classes II and III, respectively) indicating the presence or absence of circumstellar disks. There are, however, exceptions that may be borderline cases or in which IR companions may bias the YSO classification. We therefore adopted a final classification scheme that is predominantly based on the accretion signatures (WTTS vs. CTTS) and takes the IR classification as supporting evidence in borderline cases, except for protostars for which the IR classification is the only relevant parameter. Hence, in col. 10, "0" and " 1 " stand, respectively, for a protostar of Class 0 or Class I, " 2 " for an accreting (classical) T Tau star that usually shows a Class-II IR spectrum; "3" corresponds to a weak-line or Class-III object; "4" designates a brown dwarf (spectral class equal to or later than M6.25) irrespective of its accretion signatures; and " 5 " marks the Herbig Ae/Be stars in TMC. For uncertain classifications or other object types, we use the code "9".

\section{Discussion and conclusions}

\subsection{Spectral interpretation: quality of results}

To assess the quality of our spectral interpretations, we now compare results derived from the two fit methods (DEM vs 1or 2- $T$ plasma). Figure 7 compares $N_{\mathrm{H}}$ values derived from the DEM fits (shown along the abscissa) and from the 1- or 2- $T$ fits (along the ordinate). We show results for bright spectra ( $>600 \mathrm{cts}$ for the source counts parameter in Table 4 of which typically about half are found in the PN camera) and for faint spectra ( $<600$ cts) separately. The agreement is excellent. The brighter sources show a trend toward $N_{\mathrm{H}}$ being slightly lower for the 1$T$ or 2- $T$ fit than for the DEM fit. This can be understood as follows. The DEM model assumes the presence of cool material down to $\log T=6$. This plasma accounts for some of the soft emission where the 1- or 2-T model may be subject to an EM deficit. To account for the same spectral flux, the trend is to lower $N_{\mathrm{H}}$ for the spectrum with deficient cool EM. The scatter obviously becomes larger for fainter sources, but the error bars grow accordingly so that the agreement is of similar quality (lower plot).

Figure 8 analogously compares $L_{\mathrm{X}}$. For most of the brighter stars, the agreement is excellent although there are a few cases for which the 1- or 2-T fit produces significantly higher $L_{X}$. The reason for this deviation is the following. Soft components can be added with relatively little constraints if they are sufficiently absorbed. The temperature then becomes uncertain. A cooler plasma subject to photoelectric absorption will, however, require a much larger EM to produce the same observed flux. If the cool EM is a free fit parameter, an instability may occur with $T$ converging to low values while $N_{\mathrm{H}}$ and EM grow excessively. This becomes more evident for fainter sources (lower plot). In several cases, we therefore fixed $N_{\mathrm{H}}$ at the value expected from $A_{\mathrm{V}}$ for 

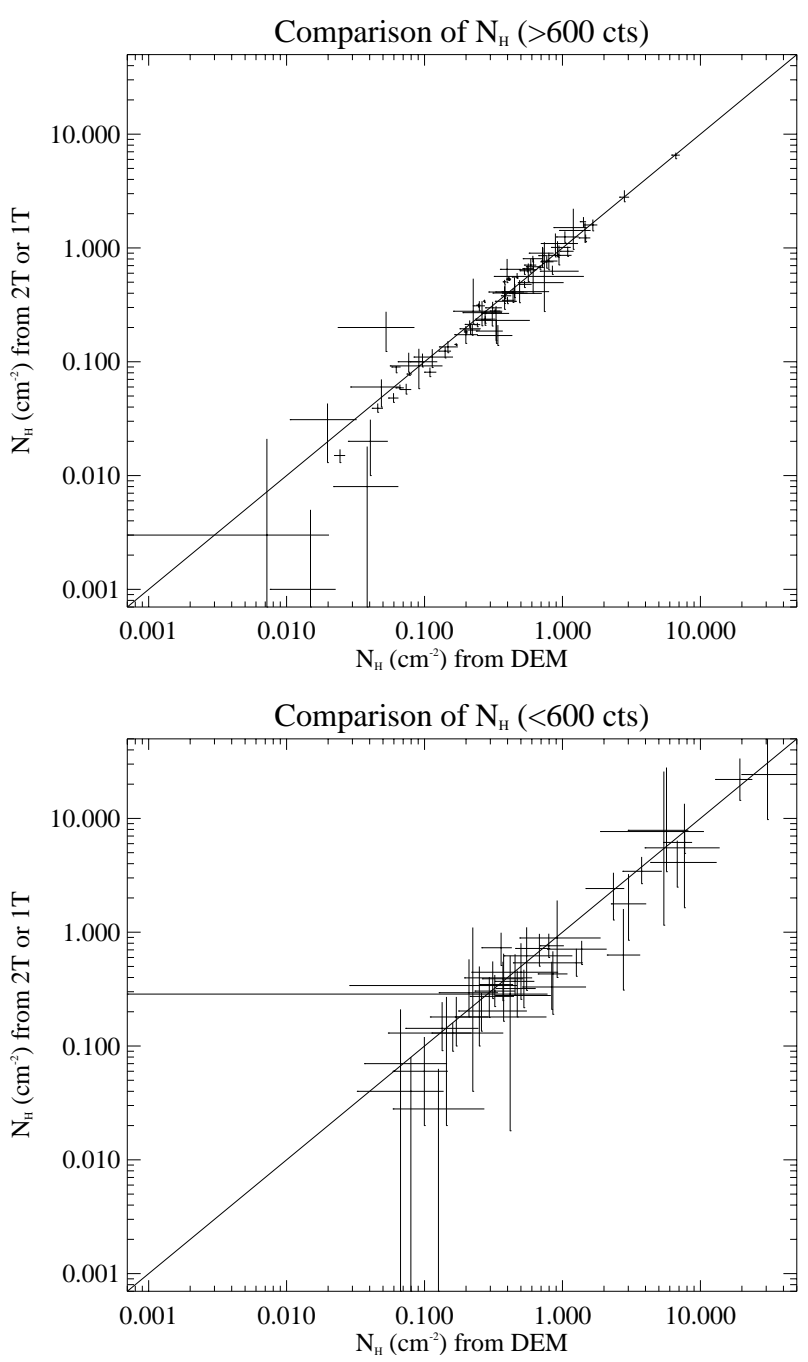

Fig. 7. Comparison of $N_{\mathrm{H}}$ values derived from the DEM method ( $x$ axis) and from 1- $T$ or 2- $T$ fits ( $y$ axis; Tables 5 and 6). Only results from $X M M$-Newton data are reported. Upper plot is for sources with more than 600 counts in total (after Table 4), lower plot for sources with fewer than 600 source counts. The solid lines indicate equal $N_{\mathrm{H}}$.

the DEM fit, whereas the 1-T fits, requiring one parameter less, were performed without this constraint. The sources that likely suffer from this numerical instability in the 1- $T$ fit are CIDA 7 (XEST-10-034), HO Tau (XEST-09-010), V410 X6 (XEST23-061), CFHT-Tau 7 (XEST-03-017), MHO 9 (XEST-22-013), and V410 A25 (XEST-23-029) that all show $L_{\mathrm{X}}$ from the $1-T$ or 2- $T$ fits at least 3 times higher compared to the DEM fit. In the former, they show $N_{\mathrm{H}}$ considerably higher than in the latter, or much higher than expected from $A_{\mathrm{V}}$, while the dominant electron temperature was very low, between $1 \mathrm{MK}$ and 3.3 MK. The first four of those six stars are faint, while MHO 9 is at the limit between bright and faint as defined for the plots in Fig. 8 .

Given that the DEM model is more rigid and avoids biases toward strongly absorbed soft emission from large amounts of cool EM, we will base most of our statistical investigations in the accompanying papers on the DEM results. This model is also more physically meaningful because work on bright spectra of nearby stars has clearly shown continuous emission measure distributions of this kind (e.g., Telleschi et al. 2005). As shown here and below, however, the results for $L_{\mathrm{X}}, N_{\mathrm{H}}$, and $T_{\mathrm{av}}$ from the two methods agree well for the brighter spectra. Caution is in order essentially only for spectra defined as "faint" here.
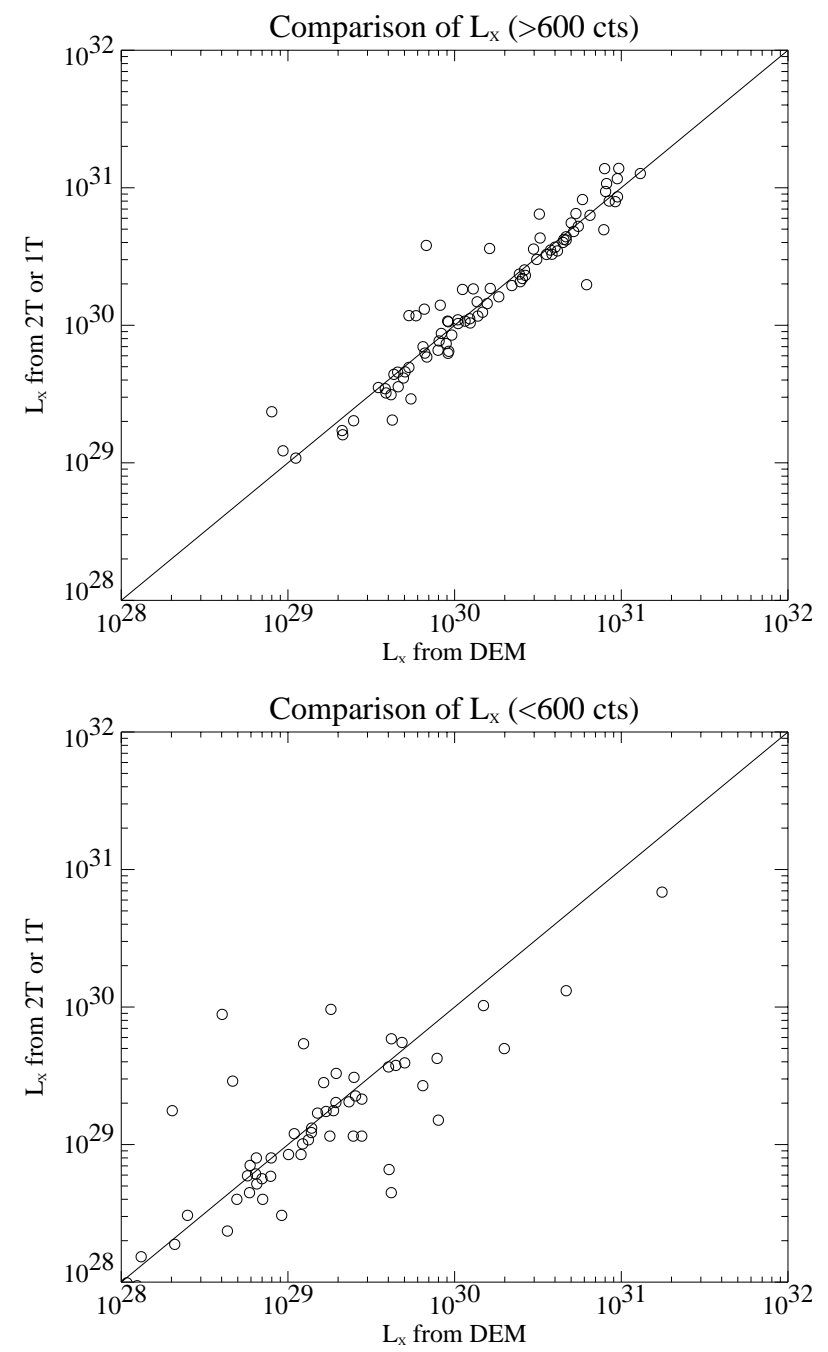

Fig. 8. Comparison of $L_{X}$ values derived from the DEM method ( $x$ axis) and from 1-T or 2-T fits ( $y$ axis; Tables 5 and 6). Only results from $X M M$-Newton data are reported. Upper plot is for sources with more than 600 counts in total (after Table 4), lower plot for sources with fewer than 600 source counts. The solid lines indicate equal $L_{X}$.

Figure 9 shows the same comparison for weakly absorbed sources (upper plot, for $N_{\mathrm{H}}<5 \times 10^{21} \mathrm{~cm}^{-2}$ ) and for more strongly absorbed sources (lower plot, for $N_{\mathrm{H}}>5 \times 10^{21} \mathrm{~cm}^{-2}$ ). It is evident that higher absorption introduces more uncertainty to the precise determination of $L_{\mathrm{X}}$. Also, these sources obviously tend to be fainter (in terms of count rates).

Figure 10 compares the average electron temperature, $T_{\mathrm{av}}$, derived from the DEM and the 2- $T$ or $1-T$ fits. Given the rather different methodology, the agreement is satisfactory for the brighter sources, while the scatter increases for faint sources, making accurate statements on electron temperatures difficult. The few outliers among the bright sources show very low average temperatures in $1-T$ or $2-T$ fits probably due to the same numerical instability described above. The worst agreement is found for the very faint spectrum of LR 1 (XEST-23-048) for which $T_{0}$ of the DEM fit is unconstrained despite fixed $\beta=-1$. In the presence of severe absorption, the DEM fit finds a low best-fit $T_{0}$, while the 1-T fit converges to a very high temperature. Both $L_{\mathrm{X}}$ are reasonable, while the DEM fit converges to a higher $N_{\mathrm{H}}$ which is preferred in the light of the very high $A_{J}$ of this star. 

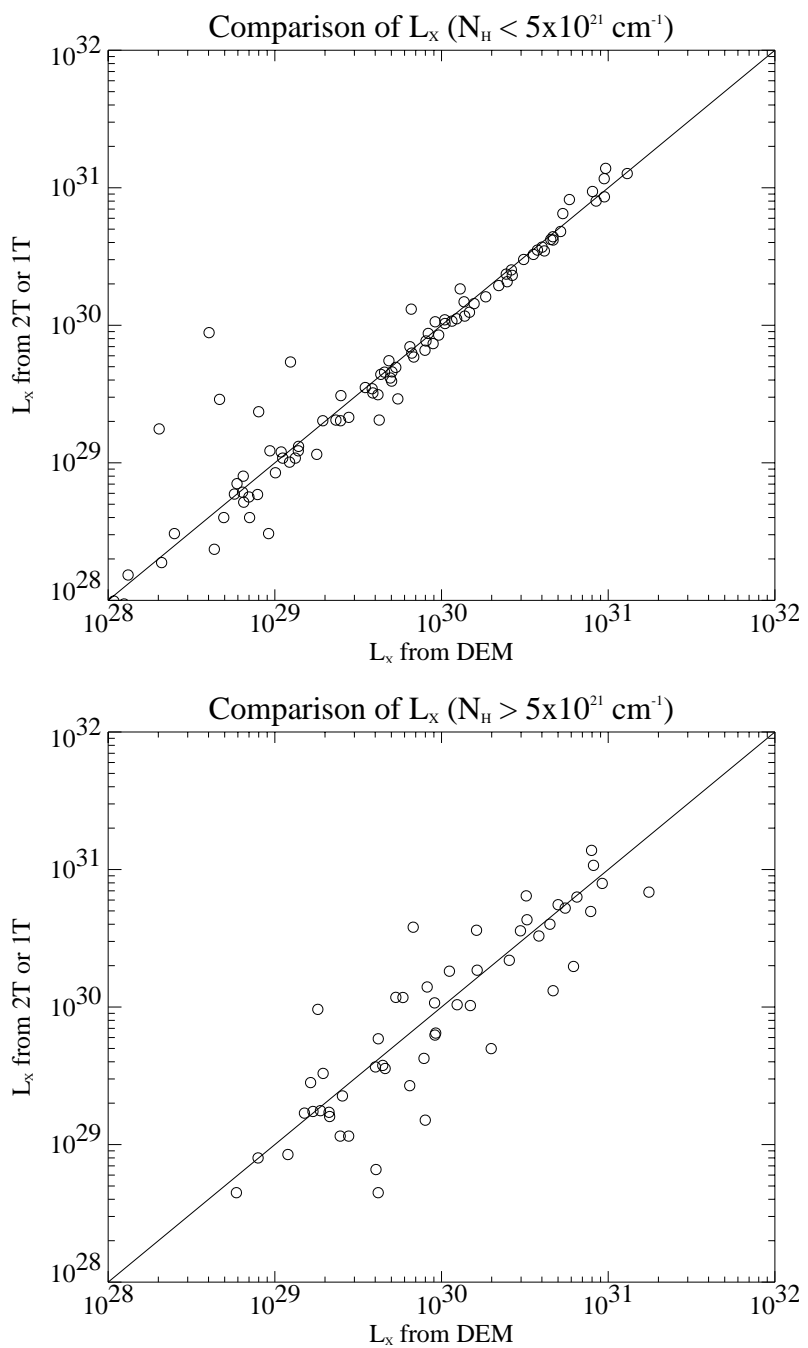

Fig. 9. Comparison of $L_{\mathrm{X}}$ values derived from the DEM method ( $x$ axis) and from 1-T or 2- $T$ fits ( $y$ axis; Tables 5 and 6). Only results from $X M M$-Newton data are reported. Upper plot is for sources with $N_{\mathrm{H}}<$ $5 \times 10^{21} \mathrm{~cm}^{-2}$, lower plot for $N_{\mathrm{H}}>5 \times 10^{21} \mathrm{~cm}^{-2}$ (based on the DEM fits in Table 5). The solid lines indicate equal $L_{\mathrm{X}}$.

Concluding from this comparison, we are confident that $N_{\mathrm{H}}$ values are meaningful within factors of about 1.5 for the brighter sample and mostly within factors of 2 for the fainter sample. $\mathrm{X}$-ray luminosities appear to be reasonably constrained within a factor of two (although with exceptions), which is a typical range of variability for most sources in any case.

\subsection{Population statistics}

Figure 11 shows an Hertzsprung-Russell Diagram (HRD) of all sources observed in the framework of this survey with isochrones and evolutionary tracks (after Siess et al. 2000) overplotted. The source symbols indicate the source type, and the symbol size corresponds to the X-ray luminosity.

The following characteristics are noteworthy: i) The surveyed sources cover a broad range of masses, from the substellar range up to $2.5-3 M_{\odot}$, the three most massive stars being HD 28867 and the Herbig stars AB Aur and V928 Tau (the latter being a multiple system). Most objects are concentrated at ages between $3 \times 10^{5}-3 \times 10^{6} \mathrm{yr}$, and very few at ages around $10^{7}$ yr. ii) As pointed out by Kenyon \& Hartmann (1995), CTTS and WTTS (Class II and III, shown in gray and white,
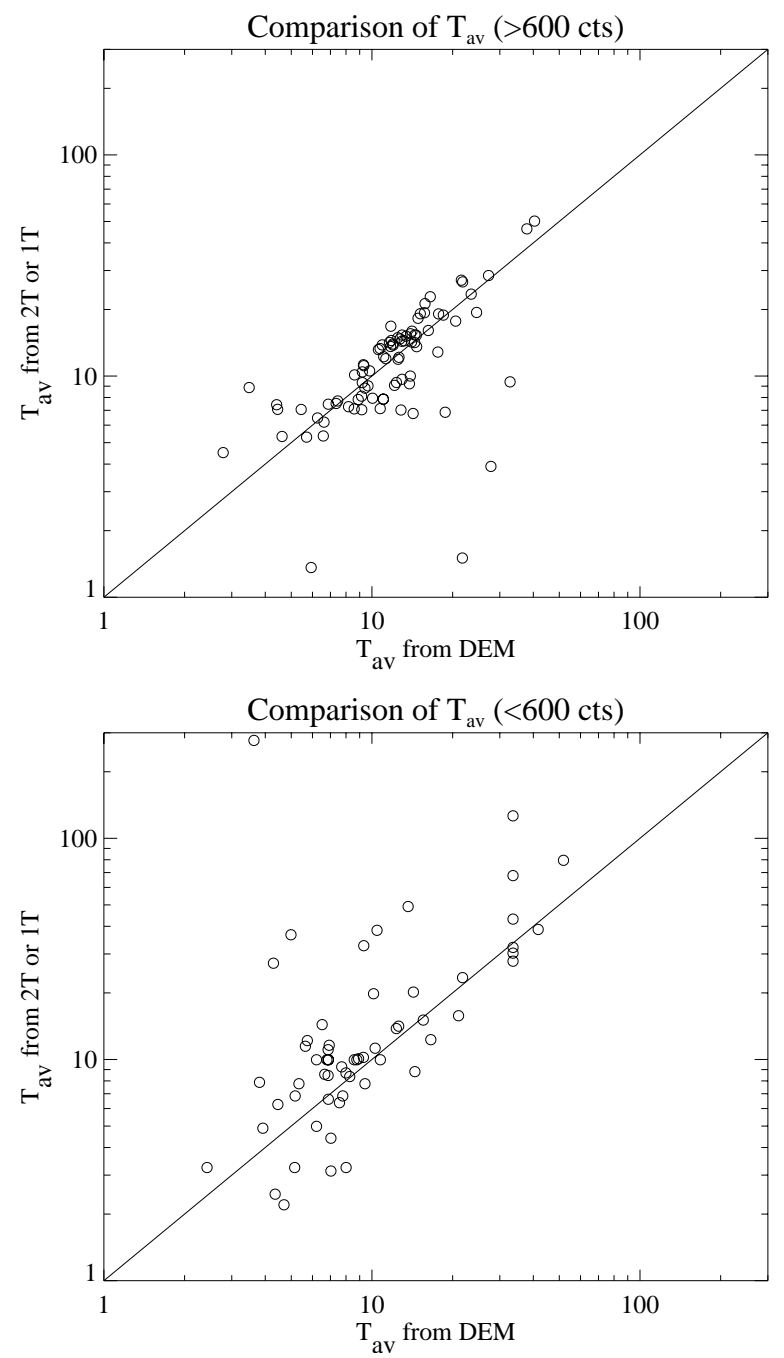

Fig. 10. Comparison of $T_{\text {av }}$ values derived from the DEM method ( $x$ axis) and from 1-T or 2- $T$ fits ( $y$ axis; Tables 5 and 6). Only results from XMM-Newton data are reported. Upper plot is for sources with more than 600 counts in total (after Table 4), lower plot for sources with fewer than 600 source counts. The solid lines indicate equal $T_{\text {av }}$.

respectively) occupy the same region in the HRD. In fact, this is also true for the detected Class-I protostars. The low-mass and substellar HRD will be more specifically discussed by Grosso et al. (2007a). iii) Most undetected sources are in the very lowmass/substellar regimes and/or have the largest isochronal ages, although for some of the latter objects, the fundamental parameters are poorly determined, as detailed below.

A few objects are apparently located below the zero-age main sequence (ZAMS) which is likely to be a consequence of poor photometry. This is particularly true for embedded sources where scattered light distorts photometric measurements. The stars below the ZAMS are, in order of increasing $T_{\text {eff }}$ : HH 30 (XEST-22-000, an edge-on star-disk system, Burrows et al. 1996), IRAS S04301+261 (XEST-18-000, a highly reddened object probably primarily detected in scattered light, Briceño et al. 2002), Haro 6-5 B = FS Tau B (XEST-11-054 = C2-1, a near-edge-on star-disk system, Padgett et al. 1999, with $A_{\mathrm{V}} \approx 10 \mathrm{mag}$ ), HBC 353 (XEST-27-000), and HBC 352 (XEST27-115). These stars will not be considered in statistical studies whenever $L_{*}$ is required (e.g., in $L_{X} / L_{*}$ ). A few stars are located between the $10 \mathrm{Myr}$ isochrone and the ZAMS, making them much older than the bulk population in Taurus. Several 
of these objects are relatively poorly studied and may suffer from inaccurate photometry. For most of them, no $A_{\mathrm{V}}$ has been given, or the extinction is large, and indeed several of them have not been detected (crosses in Fig. 11). These objects are, in order of increasing $T_{\text {eff }}$ above the $0.1 M_{\odot}$ track: ITG 33A (XEST-07-000, suggested to be an edge-on star-disk system, Martín 2000: $A_{\mathrm{V}}=3.5 \mathrm{mag}$ ), FS Tau (XEST-11-057, a strongly extincted close binary of CTTS, $A_{\mathrm{V}} \approx 5 \mathrm{mag}$ ), CoKu Tau 1 (XEST-23-000, an edge-on star-disk system, Padgett et al. 1999, $A_{\mathrm{V}}=6.8 \mathrm{mag}$ ), HN Tau (C5-2), LR 1 (XEST-23-048, with a large $\left.A_{J}=6.4 \mathrm{mag}\right), \mathrm{V} 955 \mathrm{Tau}\left(\mathrm{XEST}-10-020, A_{\mathrm{V}}=3.7 \mathrm{mag}\right)$, V410 A20 (XEST-23-000, with a large $A_{J}=6.57 \mathrm{mag}$ ), and V410 A24 (XEST-23-000, with a large $A_{J}=6.73 \mathrm{mag}-$ see Table 9 for $A_{\mathrm{V}}$ and $A_{J}$ values).

The detection statistics of our X-ray survey is summarized in Table 12 (considering only the XMM-Newton observations), based on our final classification scheme in Table 11. Here, the protostar L1551 IRS5 has been treated as a non-detection as the source closely associated with this binary has been interpreted as X-ray emission from the jets (Favata et al. 2002; Bally et al. 2003). We also list the statistics (in parentheses) if Chandra detections and non-detections are counted (note that DG Tau B and FV Tau/c have been counted as non-detections in the XMMNewton sample, while they are detections with Chandra). An important point for further statistical studies is that the X-ray sample of detected CTTS and WTTS is nearly complete for the surveyed fields (as far as the population is known). Most of the few remaining, undetected objects are considerably extincted and by implication X-ray absorbed: IRAS S04301+261 $=$ XEST- $18-000$ with $A_{J} \approx 1.8$, and CoKu Tau $1=$ XEST23-000, CFHT-Tau 19 = XEST-11-000, FV Tau/c = XEST-02000, CFHT-Tau 20 = XEST-13-000, ITG33 A = XEST-07-000, CFHT-Tau 8 = XEST-07-000, and HH $30=$ XEST-22-000 with $A_{\mathrm{V}}=6.8,7.3,3.25,3.6,3.5,1.8$, and $\approx 3 \mathrm{mag}$ and CFHT-Tau $12=$ XEST-17-000 with $A_{J}=3.44$ mag, where the $A_{\mathrm{V}}$ of IRAS S04301+261, CoKu Tau 1, HH 30, ITG 33A may have been severely underestimated owing to scattered light and strong extinction by near-edge-on disks (see above). FV Tau/c was, however, detected in a Chandra observation (C3-1) reported here, with an unexpectedly high photoelectric absorption corresponding to $N_{\mathrm{H}} \approx 10^{23} \mathrm{~cm}^{-2}$. Some of the non-detections are very-low mass stars with late spectral types (CFHT-Tau $19=$ XEST-11000, CFHT-Tau 20 = XEST-13-000, CFHT-Tau 12 = XEST-17000, and CFHT-Tau 8 = XEST-07-000 with spectral types of M5.25, M5.5, M6, and M5.5, respectively). Very little is known about IRAS 04108+2803 A. Its companion, a Class I object, was detected, its light curve showing a strong flare and its spectrum revealing very strong absorption. Perhaps, both objects are subject to similar absorption. The only formally undetected WTTS in the XMM-Newton sample is HBC 353, but it is located on the wings of HBC 352 = XEST-27-115, falls into a CCD gap of the $\mathrm{PN}$ detector, and is located far off-axis, elevating the corresponding detection limit to $\approx 180$ cts. From Table 8 , we derive that only 3 out of 45 multiple systems remain undetected (2/47 including Chandra results), while 29 out of 114 single stars are undetected (31/122 including Chandra results). In total, 45 of the 159 systems surveyed by XMM-Newton are multiple (47/169 including Chandra results).

Previous X-ray surveys of TMC did not detect the intrinsically fainter TTS population. Those previous surveys covered a wider field in Taurus (e.g., the ROSAT surveys by Neuhäuser et al. 1995; Stelzer \& Neuhäuser 2001) but were about ten times less sensitive and were confined to soft sources. Stellar classes that were thus inaccessible by these earlier surveys but are
Table 12. XEST X-ray detection statistics.

\begin{tabular}{|c|c|c|c|c|c|c|c|}
\hline \multicolumn{2}{|c|}{ Object type } & \multicolumn{2}{|c|}{$\begin{array}{l}\text { Members } \\
\text { surveyed }\end{array}$} & \multicolumn{2}{|c|}{ Detections } & \multicolumn{2}{|c|}{$\begin{array}{l}\text { Detection } \\
\text { fraction }\end{array}$} \\
\hline 0,1 & Protostars & 20 & (21) & 8 & $(10)$ & $40 \%$ & $(48 \%)$ \\
\hline 2 & CTTS & 65 & (70) & 55 & (60) & $85 \%$ & $(86 \%)$ \\
\hline 3 & WTTS & 50 & (52) & 49 & $(50)$ & $98 \%$ & $(96 \%)$ \\
\hline 4 & BDs & 16 & (17) & 8 & (9) & $50 \%$ & $(53 \%)$ \\
\hline 5 & Herbig & 2 & & 2 & & $100 \%$ & \\
\hline \multirow[t]{2}{*}{9} & others/unident. & 6 & (7) & 4 & (5) & $67 \%$ & $(71 \%)$ \\
\hline & Total & 15 & 169) & 126 & $(136)$ & $79 \%$ & $(80 \%)$ \\
\hline
\end{tabular}

\section{Notes:}

Numbers in parentheses include Chandra observations. Source near L1551 IRS5 not considered (non-detection for XEST-22-040)

systematically (albeit not completely) detected in XEST are: i) strongly absorbed, embedded protostars or T Tau stars absorbed by their own disks seen nearly edge-on; their non-detection in previous surveys is little surprising given the strong photoelectric absorption. Most of the detected protostars show no X-ray counts below $2 \mathrm{keV}$. ii) Low- $L_{\mathrm{X}}$ brown dwarfs; in XEST, the detection rate of BDs (50\% for the XMM-Newton sample, $53 \%$ if one detection from Chandra is added) is high; the remaining objects of this class are likely to be intrinsically fainter than our detection limit rather than being excessively absorbed by gas $\left(A_{\mathrm{V}}\right.$ of those objects typically being no more than a few magnitudes; see Grosso et al. 2007a for further discussion). iii) Several T Tau stars with double-peaked X-ray spectra in which a strongly absorbed hard component is accompanied by a soft excess (Güdel et al. 2007b).

The incomplete statistics in previous surveys potentially introduces bias into statistical correlations and population studies. On the other hand, we emphasize that the so far cataloged population of Taurus members may not be complete either. In particular, WTTS are predominantly detected by their X-rays (e.g., Neuhäuser et al. 1995). The WTTS statistics in Taurus may thus be incomplete in particular with regard to faint X-ray sources or WTTS located behind the TMC. Several dozen new TMC member candidates are indeed reported from XEST (Scelsi et al. 2007) but will require spectroscopic follow-up observations to confirm membership.

\subsection{Statistical properties and correlations}

Table 13 summarizes statistical properties of the surveyed sources, providing median, mean, and standard deviation of the sample for various parameters. The sample sizes vary (given in the last column, N) because not all parameters are available for all sources (see details in the footnotes of the table). The salient features of this compilation are the following: i) The agreement between the sample X-ray parameters derived from the DEM model ("DEM" in column 2) and the 1- $T$ or 2- $T$ model ("2$T$ " in column 2) is excellent. ii) Characteristic electron temperatures are around 6-8 $\mathrm{MK}$ as characterized by $T_{0}$ in the DEM model and by $T_{1}$ in the 1-T or 2-T model. The hotter component shows a mean and median around $23 \mathrm{MK}$. iii) The large majority of the sample shows $-4 \lesssim \log L_{\mathrm{X}} / L_{*} \lesssim-3$. iv) Protostars are, as expected, significantly more extincted in the visual band $\left(A_{\mathrm{V}}\right)$ in our sample than T Tau stars; among the latter, objects of type 2 (CTTS, Class-II objects) are more strongly extincted than objects of type 3 (WTTS, Class III). The median extinction of the detected sources is $\approx 1.4 \mathrm{mag}$, corresponding to a hydrogen column density of $\log N_{\mathrm{H}} \approx 21.44$, close to the measured median of $\log N_{\mathrm{H}} \approx 21.5-21.6$. iv) As pointed out on the 


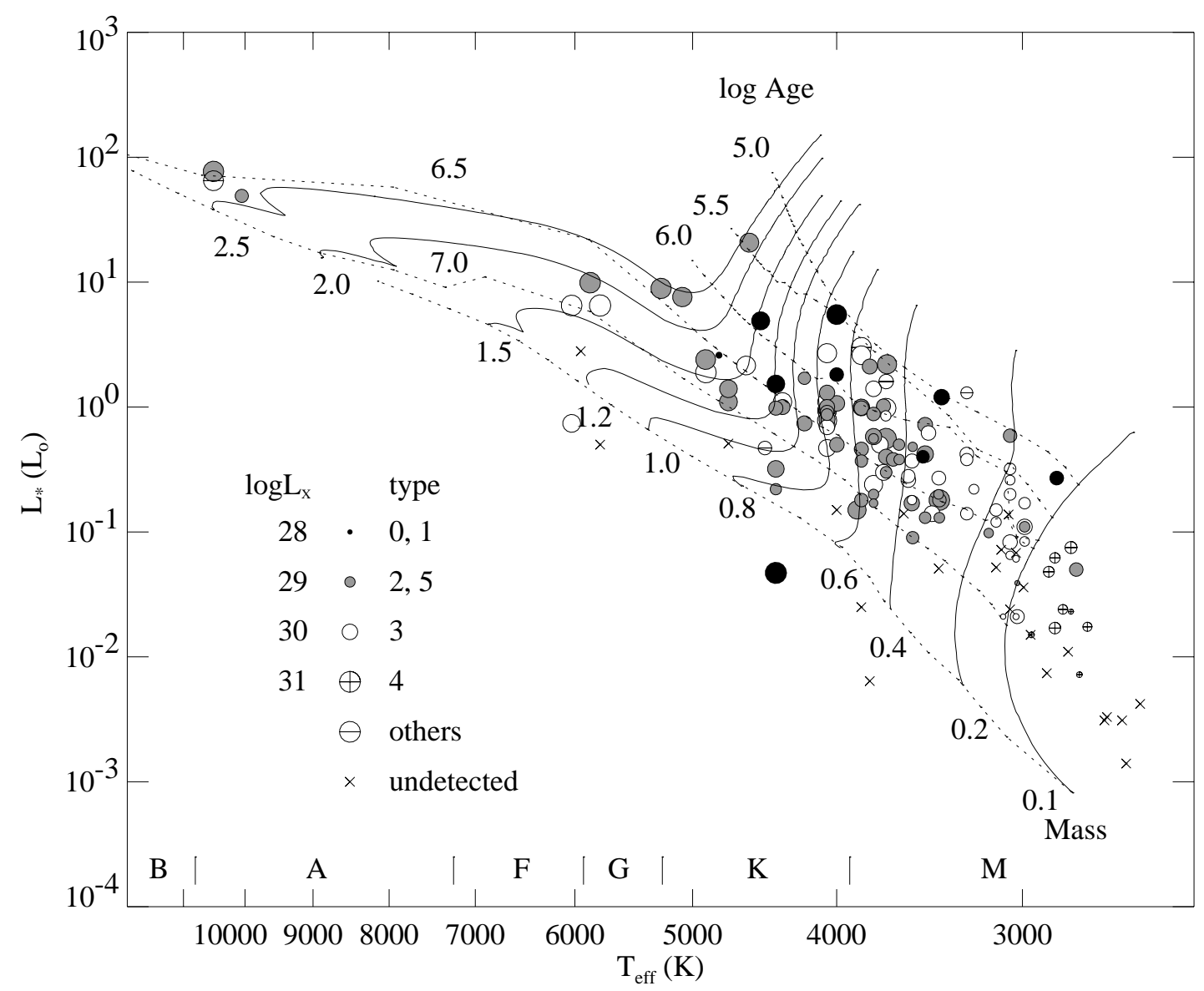

Fig. 11. HRD of all observed TMC objects for which $L_{*}$ and $T_{\text {eff }}$ are known. Symbol indicates object type (see panel in lower left corner for the key), and symbol size scales with $\log L_{X}$, crosses marking undetected sources. Additional sources from Chandra have been included, but note that the L1551 IRS5 protostar is marked here as a non-detection. Solid lines show evolutionary tracks toward the zero-age main sequence (ZAMS) for masses as given, in units of the solar mass (ZAMS). Dotted curves indicate isochrones at ages given (after Siess et al. 2000). The lowest of these curves marks the ZAMS. The spectral class ranges given at the bottom of the figure refer to main-sequence stars.

HRD, the $L_{*}$ distributions of objects of type 1,2, and 3 strongly overlap, although a marginal trend is seen toward a decreasing sample mean of $L_{*}$ with increasing type, perhaps reflecting some overall evolutionary trend. v) A wide range of accretion rates is covered (for protostars and CTTS), with a mean and median around $10^{-8} M_{\odot} \mathrm{yr}^{-1}$.

In Fig. 12 we characterize the detected stellar population by plotting $L_{\mathrm{X}}$ (from the DEM models) as a function of mass $M$ for all observed stars and substellar objects with known masses, although we exclude the following objects: DG Tau A (XEST-02022), GV Tau (XEST-13-004), DP Tau (XEST-10-045), and CW Tau (XEST-20-046) have composite X-ray spectra that may originate from two unrelated sources (Güdel et al. 2007b). L1551 IRS5 is a deeply embedded protostar, while the X-ray source seen close to it is only lightly absorbed and may be related to the jet (Favata et al. 2002; Bally et al. 2003). Finally, the light curves of DH Tau (XEST-15-040), FS Tau (XEST-11-057), and V830 Tau (XEST-04-016) showed a decay presumably from large flares. For objects that were observed and detected twice, the logarithmic average of $L_{\mathrm{X}}$ has been adopted. Although for pre-main sequence stars there is no strict correlation between $L_{*}$ and $M$, it is interesting that we find a rather well-developed correlation between $L_{\mathrm{X}}$ and $M$. The correlation coefficient for the $\log L_{\mathrm{X}}$ vs. $\log M$ sample is $C=0.79$ for 99 data points. For the subsample of type 2 ("CTTS") and type 3 objects ("WTTS"), we find $C=0.75$ and $C=0.85$ for 45 and 43 data points, respectively. All values point to a very significant correlation. We find a linear regression, $\log L_{\mathrm{X}}=(1.54 \pm 0.12) \log M+30.31 \pm 0.06$. The slopes for type 2 and type 3 objects are, respectively, $1.52 \pm 0.21$ and $1.78 \pm 0.17$. While these regressions are not significantly different, we note the larger errors for type 2 objects, and also their lower correlation coefficient, which is due to a larger scatter of $L_{\mathrm{X}}$ at a given mass. Similar trends have been noted in Orion (Preibisch et al. 2005), with $\log L_{\mathrm{X}}=(1.44 \pm 0.10) \log M+$ $30.37 \pm 0.06$ for the entire sample. This coincidence between XEST and COUP reveals that in TMC and ONC, the basic $X$ ray production mechanism scales precisely the same way with the most basic property of the stellar objects, namely their mass, regardless of any differences in age distribution, star-formation mode, or the presence or (near-) absence of high-mass stars. Part of this correlation might be explained by higher-mass stars being larger, i.e., providing more surface area for coronal active regions. Assuming similar average internal stellar density, the correlation between surface area and $L_{X}$ is, however, considerably weaker than the trend shown in Fig. 12, so that additional effects may play a role (convection zone depth, convective mass, etc).

The figure shows another important feature of our survey. Almost all sources plotted are at $L_{X}$ considerably higher than our approximate detection limit of $\approx 10^{28} \mathrm{erg} \mathrm{s}^{-1}$. There are hardly any objects in the range of $(1-5) \times 10^{28} \mathrm{erg} \mathrm{s}^{-1}$ although they would have been detected. Exceptions are the brown dwarfs. This again testifies to the near-completeness of our survey within the observed fields. 
Table 13. XEST parameter statistics.

\begin{tabular}{|c|c|c|c|c|c|}
\hline Parameter & $\overline{\text { Sample }^{a}}$ & Median & Mean & $\begin{array}{r}\text { Std. } \\
\text { dev. }^{b}\end{array}$ & $\bar{N}$ \\
\hline \multicolumn{6}{|c|}{ X-ray parameters } \\
\hline$\beta^{c}$ & DEM & -1.25 & -1.49 & 0.95 & 99 \\
\hline $\log T_{0}^{c}[\mathrm{~K}]$ & DEM & 6.90 & 6.90 & 0.28 & 119 \\
\hline $\log T_{1}^{c, d}[\mathrm{~K}]$ & $2-T$ & 6.79 & 6.76 & 0.21 & 94 \\
\hline $\log T_{2}^{c, d}[\mathrm{~K}]$ & $2-T$ & 7.37 & 7.38 & 0.25 & 105 \\
\hline \multirow[t]{2}{*}{$\log T_{\mathrm{av}}^{e}[\mathrm{~K}]$} & DEM & 7.04 & 7.03 & 0.24 & 123 \\
\hline & $2-T$ & 7.05 & 7.08 & 0.32 & 125 \\
\hline \multirow{2}{*}{$\log N_{\mathrm{H}}^{d}\left[\mathrm{~cm}^{-2}\right]$} & DEM & 21.57 & 21.52 & 0.67 & 123 \\
\hline & $2-T$ & 21.53 & 21.50 & 0.65 & 123 \\
\hline \multirow{2}{*}{$\log L_{\mathrm{X}}^{e}\left[\mathrm{erg} \mathrm{s}^{-1}\right]$} & DEM & 29.82 & 29.76 & 0.77 & 123 \\
\hline & $2-T$ & 29.80 & 29.72 & 0.78 & 125 \\
\hline \multirow[t]{2}{*}{$\log L_{\mathrm{X}} / L_{*}^{e, f}$} & DEM & -3.56 & -3.55 & 0.50 & 116 \\
\hline & $2-T$ & -3.56 & -3.58 & 0.56 & 118 \\
\hline \multicolumn{6}{|c|}{ Fundamental parameters } \\
\hline \multirow[t]{5}{*}{$A_{\mathrm{V}}\left[\mathrm{cm}^{-2}\right]$} & all & 1.46 & 2.80 & 3.38 & 116 \\
\hline & 1 & 10.20 & 9.52 & 1.93 & 6 \\
\hline & 2 & 1.95 & 3.30 & 3.61 & 57 \\
\hline & 3 & 0.87 & 1.36 & 1.56 & 38 \\
\hline & $\operatorname{det}$ & 1.35 & 2.60 & 3.36 & 97 \\
\hline \multirow[t]{5}{*}{$\log {L_{*}^{f, g}}^{\left.f, L_{\odot}\right]}$} & all & -0.34 & -0.46 & 0.82 & 146 \\
\hline & 1 & 0.08 & -0.01 & 0.49 & 16 \\
\hline & 2 & -0.25 & -0.33 & 0.61 & 60 \\
\hline & 3 & -0.43 & -0.44 & 0.59 & 46 \\
\hline & det & -0.30 & -0.33 & 0.71 & 119 \\
\hline \multirow[t]{4}{*}{$\log (\text { age })^{f, h}[\mathrm{Myr}]$} & all & 0.39 & 0.38 & 0.46 & 112 \\
\hline & 1 & 0.05 & 0.23 & 0.54 & 9 \\
\hline & 2 & 0.43 & 0.44 & 0.42 & 52 \\
\hline & 3 & 0.39 & 0.32 & 0.35 & 43 \\
\hline \multirow[t]{4}{*}{$\log (\text { mass })^{f, g}\left[M_{\odot}\right]$} & all & -0.29 & -0.37 & 0.43 & 123 \\
\hline & 1 & -0.17 & -0.23 & 0.22 & 10 \\
\hline & 2 & -0.25 & -0.27 & 0.31 & 54 \\
\hline & 3 & -0.39 & -0.40 & 0.34 & 43 \\
\hline \multirow[t]{4}{*}{$\log (P)^{i}[\mathrm{~d}]$} & all & 0.69 & 0.64 & 0.28 & 69 \\
\hline & 1 & 0.63 & 0.54 & 0.32 & 10 \\
\hline & 2 & 0.77 & 0.73 & 0.21 & 35 \\
\hline & 3 & 0.58 & 0.56 & 0.33 & 22 \\
\hline $\log (\dot{M})^{j}\left[M_{\odot} \mathrm{yr}^{-1}\right]$ & all & -8.08 & -8.20 & 1.21 & 57 \\
\hline
\end{tabular}

Notes: Only results from XMM-Newton have been considered.

For multiple detections, parameters were averaged.

${ }^{a}$ DEM: from DEM fit; 2- $T$ : from 1- $T$ or 2- $T$ fit; all; all objects;

$1,2,3$; object types; det $=$ X-ray detected objects.

${ }^{b}$ Standard deviation of distribution.

${ }^{c}$ Only non-fixed fit parameters for detections considered.

${ }^{d}$ Only non-zero values for detections considered.

${ }^{e}$ Only detections considered.

${ }^{f}$ Only for known $L_{*}$ above the ZAMS.

${ }^{g}$ For multiples, value for primary if available.

${ }^{h}$ For multiples, logarithmic average of components if available.

${ }^{i}$ Upper limits are adopted as measured values.

${ }^{j}$ Average of reported range if at least one non-upper limit given.

Figure 13 shows the distribution of the $L_{\mathrm{X}}$ (derived from the DEM models) as a function of the (stellar, photospheric) bolometric luminosity $L_{*}$ for all spectrally modeled TTS and protostars, and also including BDs. We again exclude the peculiar spectral sources with two absorbers, the protostar L1551 IRS5, the three stars with gradually decaying light curves $(\mathrm{DH}$ Tau $=$ XEST-15-040, FS Tau = XEST-11-057, V830 Tau = XEST04-016), and also all sources located below the ZAMS in the HRD. For stars observed and detected twice, we again plot the logarithmic average of $L_{\mathrm{X}}$. Most stars cluster between $L_{\mathrm{X}} / L_{*}=10^{-4}-10^{-3}$ as is often found in star-forming regions

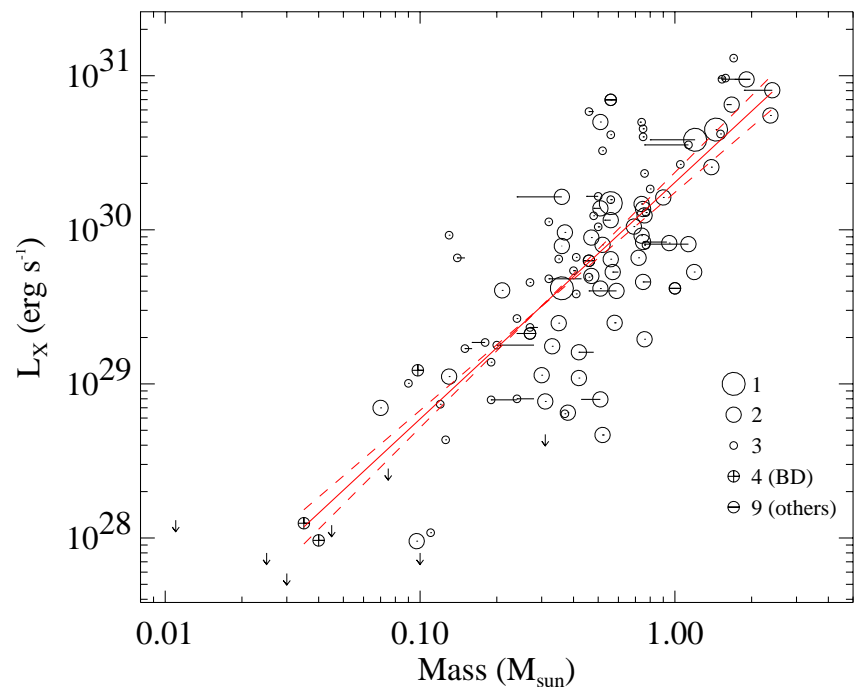

Fig. 12. X-ray luminosity $L_{\mathrm{X}}$ (based on DEM fits) vs. stellar mass $M$ for all detected XEST sources (Chandra sources and Herbig stars are not included), or upper limits (arrows). For multiple systems, the primary mass has been used if available. Symbols defining the object type are given in the lower right corner. The horizontal bars for some objects show the ranges of masses derived from literature $L_{*}$ and $T_{\text {eff }}$, while the circle is centered at the adopted $M$. The straight line gives a linear regression using the logarithmic values for the $\mathrm{X}$-ray detections, and the $1 \sigma$ ranges for the slopes: $\log L_{\mathrm{X}}=(1.54 \pm 0.12) \log M+30.31 \pm 0.06$.

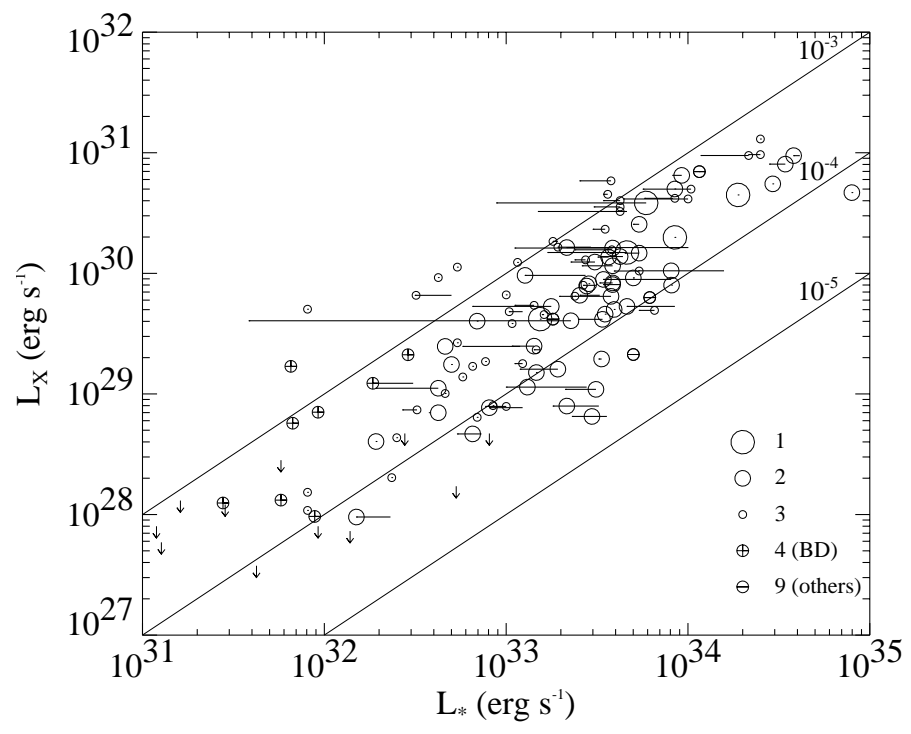

Fig. 13. X-ray luminosity $L_{\mathrm{X}}$ (based on DEM fits) vs. stellar luminosity $L_{*}$ for all X-ray detected XEST sources (Chandra sources, Herbig stars, and objects "below" the ZAMS not included), or upper limits (arrows). For multiple systems, total ("system") luminosity $L_{*}$ has been used. Symbols defining the object type are given in the lower right corner. The horizontal bars for some objects show the ranges of literature values for $L_{*}$, while the circle marks the adopted $L_{*}$.

(see Preibisch et al. 2005 for a similar analysis for the Orion sample). The value $L_{\mathrm{X}} / L_{*}=10^{-3}$ corresponds to the saturation value for rapidly rotating main-sequence stars. One key parameter that drives the X-ray output is obviously $L_{*}$.

We finally present the X-ray luminosity function (XLF) of our Taurus sample in Fig. 14. The XLF has been calculated using the Kaplan-Meier estimator in the ASURV software package (Lavalley et al. 1992) that considers upper limits to $L_{X}$ also. Nondetections for which no reasonable upper limit can be given were 


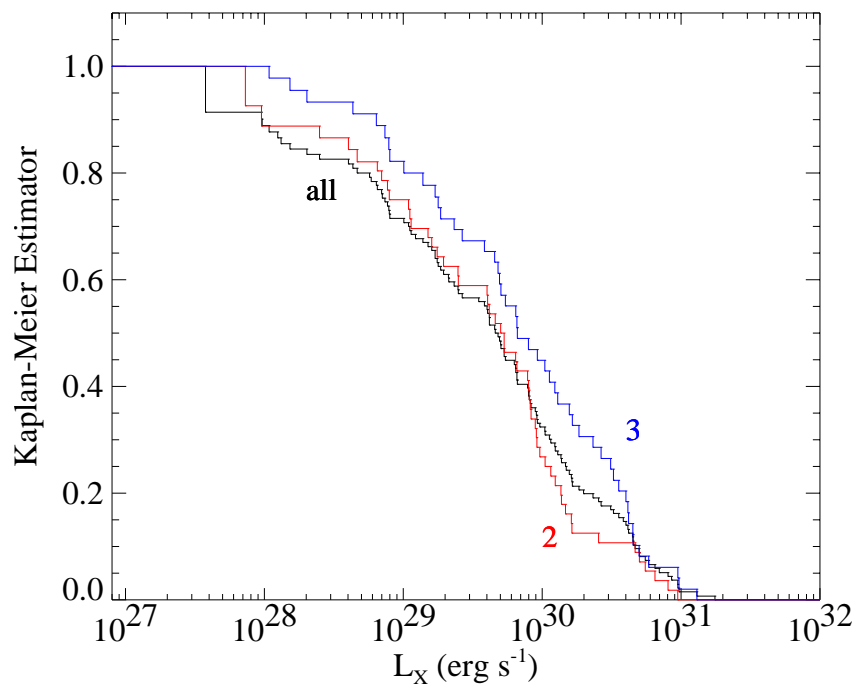

Fig. 14. X-ray luminosity function for the entire sample (black), type 2 objects (red), and type 3 objects (blue).

not included. We also dropped the same non-standard X-ray sources excluded above (double-absorber spectra, L1551 IRS 5 , and sources with gradual flare decays), but include BDs and Herbig stars, thus using $136 \mathrm{X}$-ray luminosity values, including 17 upper limits. We also plot separately the XLF for type 2 (CTTS, 56 values, including 6 upper limits) and type 3 (WTTS, 49 values, 1 upper limit). We note the shift of the CTTS distribution toward lower luminosities compared to WTTS, by a factor of about two. This is also evident for $L_{\mathrm{X}} / L_{\mathrm{bol}}$ (Telleschi et al. 2007a). A two-sample test based on the Wilcoxon test and the logrank test performed in ASURV indicates a probability of only 6-9\% that the two distributions are drawn from the same parent population. A more detailed discussion on distinctions between CTTS and WTTS is given by Telleschi et al. (2007a).

\section{Outlook}

The XEST project gives near-complete access to the entire pre-main sequence population of the Taurus Molecular Clouds within the $\approx 5$ sq. degrees covered by this survey. Essentially all WTTS and nearly all CTTS have been detected, exceptions being a few low-mass, strongly absorbed sources. About half of the embedded protostellar sample is detected, and a similar fraction of the BDs. This survey thus goes deeper than previous X-ray surveys of Taurus (e.g., Neuhäuser et al. 1995; Stelzer $\&$ Neuhäuser 2001) by about an order of magnitude for lowextinction objects and provides systematic access to the protostellar and the brown-dwarf samples for the first time. The survey forms the basis for more specialized studies as discussed in the accompanying series of papers.

Furthermore, the survey will provide a useful database for correlation studies at other wavelengths. In particular, gas-todust ratio studies and investigations of the effects of accretion disks on magnetic fields in pre-main sequence systems will be undertaken in conjunction with a wide-field survey available from the Spitzer Space Telescope (Padgett et al. 2007).

Acknowledgements. The XEST project was made possible by the co-operation of many individuals. In particular, we thank the XMM-Newton SOC team in Vilspa for its excellent support before, during, and after the observations. We warmly thank the International Space Science Institute (ISSI) in Bern for their financial support of the project and their hospitality during several XEST team meetings at ISSI. Thanks go in particular to Vittorio Manno, Brigitte Fasler, and
Saliba F. Saliba for their efforts to make these events enjoyable. In the course of the scientific analysis, we obtained advice from numerous colleagues, of which we mention in particular Claude Catala, Laurence DeWarf, Ed Fitzpatrick, Sylvain Guieu, Antonio Maggio, and Karl Stapelfeldt. Francesco Damiani is thanked for giving us access to his private version of PWXDETECT for XMMNewton data. We are grateful to the referee, Marc Gagné, for his competent review of this paper and his constructive comments that helped improve the paper. This research is based on observations obtained with XMM-Newton, an ESA science mission with instruments and contributions directly funded by ESA member states and the USA (NASA). This publication makes use of data products from the Two Micron All Sky Survey (2MASS), which is a joint project of the University of Massachusetts and the Infrared Processing and Analysis Center/California Institute of Technology, funded by the National Aeronautics and Space Administration and the National Science Foundation. Further, our research has made use of the SIMBAD database, operated at CDS, Strasbourg, France. We have made use of the ASURV statistical software package maintained by Penn State. X-ray astronomy research at PSI has been supported by the Swiss National Science Foundation (grants 20-66875.01 and 20-109255/1). MA acknowledges support by NASA grants NNG05GF92G. The Palermo group acknowledges financial contributions from contract ASI-INAF I/023/05/0.

\section{References}

Alexander, R. D., Clarke, C. J., \& Pringle, J. E. 2004, MNRAS, 354, 71

Anders, E., \& Grevesse, N. 1989, Geochim. Cosmochim. Acta, 53, 197 (AG89) Andrews, S. M., \& Williams, J. P. 2005, ApJ, 631, 1134

Argiroffi, C., Drake, J. J., Maggio, A., et al. 2004, ApJ, 609, 925

Arnaud, K. A. 1996, in ASP Conf. Ser., 101, Astronomical Data Analysis Software and Systems V, ed. G. Jacoby, \& J. Barnes (San Francisco: ASP), 17

Arzner, K., Güdel, M., Briggs, K. R., Telleschi, A., \& Audard, M. 2007a, A\&A, 468,477

Arzner, K., Güdel, M., Briggs, K. R., et al. 2007b, A\&A, 468, 501

Audard, M., Briggs, K. R., Grosso, N., et al. 2007, A\&A, 468, 379

Balbus, S. A., \& Hawley J. F. 1991, ApJ, 376, 214

Bally, J., Feigelson, E., \& Reipurth, B. 2003, ApJ, 584, 843

Barrado y Navascués, D., \& Martín, E. L. 2003, AJ, 126, 2997

Berghoefer, T. W., Schmitt, J. H. M. M., \& Cassinelli, J. P. 1996, A\&AS, 118, 481

Böhm, T., \& Catala, C. 1993, A\&AS, 101, 629

Bouvier, J. 1990, AJ, 99, 946

Bouvier, J., 2006, private communication

Briceño, C., Calvet, N., Gomez, M., et al. 1993, PASP, 105, 686

Briceño, C., Hartmann, L., Stauffer, J., \& Martín, E. 1998, AJ, 115, 2074

Briceño, C., Luhman, K. L., Hartmann, L., Stauffer, J. R., \& Kirkpatrick, J. D. 2002, ApJ, 580, 317

Briggs, K. R., Güdel, M., Telleschi, A., et al. 2007, A\&A, 468, 413

Burrows, C. J., Stapelfeldt, K. R., Watson, A. M., et al. 1996, ApJ, 473, 437

Calvet, N., Muzerolle, J., Briceño, C., et al. 2004, AJ, 128, 1294

Carpenter, J. M. 2000, AJ, 120, 3139

Cohen, M., \& Kuhi, L. V. 1979, ApJS, 41, 743

Cutri, R. M., et al. 2003, The IRSA 2MASS All-Sky Point Source Catalog,

NASA/IPAC Infrared Science Archive.

http://irsa.ipac.caltech.edu/applications/Gator/

Dame, T. M., Ungerechts, H., Cohen, R. S., et al. 1987, ApJ, 322, 706

Damiani, F., \& Micela, G. 1995, ApJ, 446, 341

Damiani, F., Micela, G., Sciortino, S., \& Harnden, F. R. Jr. 1995, ApJ, 446, 331

Damiani, F., Maggio, A., Micela, G., \& Sciortino, S. 1997, ApJ, 483, 350

den Herder, J. W., Brinkman, A. C., Kahn, S. M., et al. 2001, A\&A, 365, L7

DeWarf, L. E., \& Fitzpatrick, E. 2006, private communication

DeWarf, L. E., Sepinsky, J. F., Guinan, E. F., Ribas, I., \& Nadalin, I. 2003, ApJ, 590,357

Dobashi, K., Uehara, H., Kandori, R., et al. 2005, PASJ, 57, 1

Dolan, C. J., \& Mathieu, R. D. 2001, AJ, 1212124

Doppmann, G. W., Greene, T. P., Covey, K. R., \& Lada, C. J. 2005, AJ, 130, 1145

Duchêne, G. 1999, A\&A, 341, 547

Duchêne, G., Monin, J.-L., Bouvier, J., \& Ménard, F. 1999, A\&A, 351, 954

Duchêne, G., Ghez, A. M., \& McCabe, C. 2002, ApJ, 568, 771

Duchêne, G., Ménard, F., Stapelfeldt, K., \& Duvert, G. 2003, A\&A, 400, 559

Duchêne, G., Bouvier, J., Bontemps, S., André, P., \& Motte, F. 2004, A\&A, 427, 651

Dutrey, A., Guilloteau, S., Duvert, G., et al. 1996, A\&A, 309, 493

Favata, F., Fridlund, C. V. M., Micela, G., Sciortino, S., \& Kaas, A. A. 2002, A\&A, 386, 204

Favata, F., Giardino, G., Micela, G., Sciortino, S., \& Damiani, F. 2003, A\&A, 403, 187

Feigelson, E. D., \& DeCampli, W. M. 1981, ApJ, 243, L89 
Feigelson, E. D. \& Kriss, G. A. 1981, Ap, 248, L35

Feigelson, E. D., \& Montmerle, T. 1999, ARA\&A, 37, 363

Feigelson, E. D., Jackson, J. M., Mathieu, R. D., Myers, P. C., \& Walter, F. M. 1987, AJ, 94, 1251

Feigelson, E. D., Townsley, L., Güdel, M., \& Stassun, K. 2007, Protostars \& Planets V, ed. B. Reipurth, D. Jewitt, \& K. Keil (Tucson: University of Arizona Press), 313

Fernández, M., Ortiz, E., Eiroa, C.,\& Miranda, L. F. 1995, A\&AS, 114, 439

Finkenzeller, U., \& Mundt, R. 1984, A\&AS, 55, 109

Flaccomio, E., Micela, G., Sciortino, S., et al. 2000, A\&A, 355, 651

Flaccomio, E., Micela, G., \& Sciortino S. 2003a, A\&A, 402, 277

Flaccomio, E., Damiani, F., Micela, G., et al. 2003b, ApJ, 582, 398

Franciosini, E., Pillitteri, I., Stelzer, B., et al. 2007, A\&A, 468, 485

Gagné, M., Skinner, S. L., \& Daniel, K. J. 2004, ApJ, 613, 393

Garcia-Alvarez, D., Drake, J. J., Lin, L., Kashyap, V. L., \& Ball, B. 2005, ApJ, 621,1009

Getman, K. V., Flaccomio, E., Broos, P. S., et al. 2005, ApJS, 160, 319

Ghez, A. M., Neugebauer, G., \& Matthews, K. 1993, AJ, 106, 2005

Ghez, A. M., White, R. J., \& Simon, M. 1997, ApJ, 490, 353

Ghizzardi, S., et al. 2002, In-flight calibration of the PSF for the PN camera, XMM-SOC-CAL-TN-0029

Glassgold, A. E., Najita, J., \& Igea, J. 2004, ApJ, 615, 972

Glauser, A. M., Ménard, F., Pinte, C., et al. 2007, A\&A, submitted

Grosso, N., Briggs, K. R., Güdel, M., et al. 2007a, A\&A, 468, 391

Grosso, N., Audard, M., Bouvier, J., et al. 2007b, A\&A, 468, 557

Güdel, M. 2002, ARA\&A, 40, 217

Güdel, M. 2004, A\&AR, 12, 71

Güdel, M., Audard, M., Kashyap, V. L., Drake, J. J., \& Guinan, E. F. 2003, ApJ, 582,423

Güdel, M., Skinner, S. L., Briggs, K. R., et al. 2005, ApJ, 626, L53

Güdel, M., Padgett, D. L., \& Dougados, C. 2007a, Protostars \& Planets V, ed. B. Reipurth, D. Jewitt, \& K. Keil (Tucson: University of Arizona Press), 329

Güdel, M., Telleschi, A., Audard, M., et al. 2007b, A\&A, 468, 515

Güdel, M., Skinner, S. L., Mel'nikov, S. Y., et al. 2007c, A\&A, 468, 529

Guieu, S. 2006, private communication

Guieu, S., Dougados, C., Monin, J.-L., Magnier, E., \& Martín, E. L. 2006, A\&A, 446,485

Hartigan, P., \& Kenyon, S. J. 2003, ApJ, 583, 334

Hartigan, P., Strom, K. M., \& Strom, S. E. 1994, ApJ, 427, 961

Hartmann, L. 2002, ApJ, 578, 914

Hartmann, L., Megeath, S. T., Allen, L., et al. 2005, ApJ, 629, 881

Hayashi, M. R., Shibata, K., \& Matsumoto, R. 1996, ApJ, 468, L37

Herbig, G. H., Vrba, F. V., \& Rydgren, A. E. 1986, AJ, 91, 575

Itoh, Y., Hayashi, M., Tamura, M., et al. 2005, ApJ, 620, 984

Jansen, F., Lumb, D., Altieri, B., et al. 2001, A\&A, 365, L1

Jensen, E. L., \& Akeson, R. L. 2003, ApJ, 584, 875

Kastner, J. H., Huenemoerder, D. P., Schulz, N. S., Canizares, C. R., \& Weintraub, D. A. 2002, ApJ, 567, 434

Kenyon, S. J., \& Hartmann, L. 1995, ApJS, 101, 117

Kenyon, S. J., Calvet, N., \& Hartmann, L. 1993, ApJ, 414, 676

Kenyon S. J., Dobrzycka D., \& Hartmann L. 1994, AJ, 108, 1872

Kenyon S. J., Brown, D. I., Tout, C. A., \& Berlind, P. 1998, AJ, 115, 2491

Kirsch, M. 2006, EPIC status of calibration and data analysis, XMM-SOC-CALTN-0018,

http://xmm. esac. esa.int/docs/documents/CAL-TN-0018.pdf

Kitamura, Y., Momose, M., Yokogawa, S., et al. 2002, ApJ, 581, 357

Kraft, R. P., Burrows, D. N., \& Nousek, J. A. 1991, ApJ, 374, 344

Kun, M. 1998, ApJS, 115, 59

Lavalley, M., Isobe, T., \& Feigelson, E. 1992, in Astronomical Data Analysis Software and Systems I, ed. D. M. Worrall, C. Biemesderfer, \& J. Barnes (San Francisco: ASP), 245

Leinert, Ch., \& Haas, M. 1989, ApJ, 342, L39

Leinert, Ch., Zinnecker, H., Weitzel, N., et al. 1993, A\&A, 278, 129

Leinert, C., Richichi, A., \& Haas, M. 1997, A\&A, 318, 472

Loinard, L., Mioduszewski, A. J., Rodríguez, L. F., et al. 2005, ApJ, 619, L179

Luhman, K. L. 2000, ApJ, 544, 1044

Luhman, K. L. 2004, ApJ, 617, 1216

Luhman, K. L. 2006, ApJ, 645, 676

Luhman, K. L., Briceño C., Stauffer, J. R., Hartmann, L., et al. 2003, ApJ, 590, 348

Martín, E. L. 2000, AJ, 120, 2114

Martín E. L., \& Magazzù, A. 1999, A\&A, 342, 173

Martín, E. L., Rebolo, R., Magazzù, A., \& Pavlenko, Ya. V. 1994, A\&A, 282, 503

Martín, E. L., Basri, G., \& Zapatero Osorio, R. 1999, AJ, 118, 1005

Martín, E. L., Dougados, C., Magnier, E., Ménard, F., Magazzù, A., Cuillandre, J.-C., \& Delfosse, X. 2001, ApJ, 561, L195

Mason, K. O., Breeveld, A., Much, R., et al. 2001, A\&A, 365, L36
McCabe, C., Ghez, A. M., Prato, L., et al. 2006, ApJ, 636, 932

Mathieu, R. D. 1994, ARA\&A, 32, 465

Mohanty, S., Jayawardhana, R., \& Basri, G. 2005, ApJ, 626, 498

Moneti, A., \& Zinnecker, H. 1991, A\&A, 242, 438

Monin, J.-L., Ménard, F., \& Duchêne, G. 1998, A\&A, 339, 113

Montmerle, T., Grosso, N., Tsuboi, Y., \& Koyama, K. 2000, ApJ, 532, 1097

Morrison, R., \& McCammon, D. 1983, ApJ, 270, 119

Mundt, R., Walter, F. M., Feigelson, E. D., et al. 1983, ApJ, 269, 229

Muzerolle, J., Hartmann, L., \& Calvet, N. 1998, AJ, 116, 2965

Muzerolle, J., Hillenbrand, L., Calvet, N., Briceño, C., \& Hartmann, L. 2003, ApJ, 592, 266

Muzerolle, J., Luhman, K. L., Briceño, C., Hartmann, L., \& Calvet, N. 2005, ApJ, 625, 906

Neuhäuser, R., Sterzik, M. F., Schmitt, J. H. M. M., Wichmann, R., \& Krautter, J. 1995, A\&A, 297, 391

Ohashi, N., Hayashi, M., Kawabe, R., \& Ishiguro, M. 1996, ApJ, 466, 317

O'Dell, C. R. 1998, AJ, 115, 263

Onishi, T., Mizuno, A., Kawamura, A., Tachihara, K., \& Fukui, Y. 2002, ApJ, 575,950

Padgett, D. L., Brandner, W., Stapelfeldt, K. R., et al. 1999, AJ, 117, 1490

Padgett, D. L., et al. 2007, in preparation

Palla, F., \& Stahler, S. W. 2002, ApJ, 581, 1194

Petr, M. G., Coude Du Foresto, V., Beckwith, S. V. W., Richichi, A., \& McCaughrean, M. J. 1998, ApJ, 500, 825

Prato, L., \& Simon, M. 1997, ApJ, 474, 455

Pravdo, S. H., Feigelson, E. D., Garmire, G., et al. 2001, Nature, 413, 708

Preibisch, T., \& Zinnecker, H. 2001, AJ, 122, 866

Preibisch, T., Kim, Y.-C., Favata, F., et al. 2005, ApJS, 160, 401

Pudritz, R. E., \& Norman, C. A. 1986, ApJ, 301, 571

Qi, C., Kessler, J. E. Koerner, D. W., Sargent, A. I., \& Blake, G. A. 2003, ApJ, 597, 986

Read, A. M., \& Ponman, T. J. 2003, A\&A, 409, 395

Rebull, L. M., Wolff, S. C., \& Strom, S. E. 2004, AJ, 127, 1029

Reipurth, B., \& Zinnecker, H. 1993, A\&A,278, 81

Reipurth, B., Rodríguez, L. F., Anglada, G., \& Bally, J. 2002, AJ, 124, 1045

Richichi, A., Leinert, Ch., Jameson, R., \& Zinnecker, H. 1994, A\&A, 287, 145

Rodmann, J., Henning, Th., Chandler, C. J., Mundy, L. G., \& Wilner, D. J. 2006, A\&A, 466, 211

Rodríguez, L. F., D’Alessio, P., Wilner, D. J., et al. 1998, Nature, 395, 355

Scelsi, L., Maggio, A., Peres, G., \& Pallavicini, R. 2005, A\&A, 432, 671

Scelsi, L., Maggio, A., Micela, G., et al. 2007, A\&A, 468, 405

Scholz, A., Jayawardhana, R., \& Wood, K. 2006, ApJ, 645, 1498

Shu, F., Najita, J., Ostriker, E., et al. 1994, ApJ, 429, 781

Siess, L., Dufour, E., \& Forestini, M. 2000, A\&A, 358, 593

Simon, M., Chen, W. P., Howell, R. R., \& Slovik, D. 1992, ApJ, 384, 212

Simon, M., Ghez, A. M., Leinert, Ch., et al. 1995, ApJ, 443, 625

Simon, M., Holfeltz, S. T., \& Taff, L. G. 1996, ApJ, 469, 890

Smith, K. W., Balega, Y. Y., Duschl, W. J., et al. 2005, A\&A, 431, 307

Stelzer, B., \& Neuhäuser, R. 2001, A\&A, 377, 538

Stelzer, B., Flaccomio, E., Micela, G., et al. 2007, A\&A, 468, 463

Strom, K. M., \& Strom, S. E. 1994, ApJ, 424, 237

Strom, K. M. Strom, S. E., Edwards, S., Cabrit, S., \& Skrutskie, M. 1989, AJ, 97, 1451

Strom, K. M., Strom, S. E., Wilkin, F. P., et al. 1990, ApJ, 362, 168

Strüder, L., Briel, U., Dennerl, K., et al. 2001, A\&A, 365, L18

Telleschi, A., Güdel, M., Briggs, K., et al. 2005, ApJ, 622, 653

Telleschi, A., Güdel, M., Briggs, K. R., Audard, M., \& Palla, F. 2007a, A\&A, 468,425

Telleschi, A., Güdel, M., Briggs, K. R., Audard, M., \& Scelsi, L. 2007b, A\&A, 468,443

Telleschi, A., Güdel, M., Briggs, K. R., et al. 2007c, A\&A, 468, 541

Turner, M. J. L., Abbey, A., Arnaud, M., et al. 2001, A\&A, 365, L27

Ungerechts, H., \& Thaddeus, P. 1987, ApJS, 63, 645

Vuong, M. H., Montmerle, T., Grosso, N., et al. 2003, A\&A, 408, 581

Walter, F. M., \& Boyd, W. T. 1991, ApJ, 370, 318

Walter, F. M., \& Kuhi, L. V. 1981, ApJ, 250, 254

Walter, F. M., Brown, A., Mathieu, R. D., Myers, P. C., \& Vrba, F. J. 1988, AJ, 96, 297

Walter, F. M., Beck, T. L., Morse, J. A., \& Wolk, S. 2003, AJ, 125, 2123

Weaver, W. B., \& Jones, G. 1992, ApJS, 78, 239

Welty, A. D. 1995, 5AJ, 110, 776

White, R. J., \& Basri, G. 2003, ApJ, 582, 1109

White, R. J., \& Ghez, A. M. 2001, ApJ, 556, 265

White, R. J., \& Hillenbrand, L. A. 2004, 616, 998

Woitas, J. 2003, A\&A, 406, 685

Woitas, J., Leinert, Ch., \& Köhler, R. 2001, A\&A, 376, 982

Wolf, S., Padgett, D., \& Stapelfeldt, K. 2003, ApJ, 588, 373

Wolk, S. J., Harnden, F. R., Jr., Flaccomio, E., et al. 2005, ApJS, 160, 423 


\section{Online Material}




\section{References cited in the tables:}

$1=$ Andrews \& Williams (2005)

$2=$ Bouvier (2006)

3 = Briceño et al. (1993)

$4=$ Briceño et al. (1998)

$5=$ Briceño et al. (2002)

$6=$ Böhm \& Catala (1993)

$7=$ Cohen \& Kuhi (1979)

$8=$ DeWarf et al. (2003)

$9=$ DeWarf \& Fitzpatrick (2006)

$10=$ Doppmann et al. (2005)

$11=$ Duchêne et al. (1999)

$12=$ Duchêne et al. (2002)

$13=$ Duchêne et al. (2004)

14 = Fernández et al. (1995)

$15=$ Finkenzeller \& Mundt (1984)

$16=$ Ghez et al. (1993)

$17=$ Ghez et al. (1997)

$18=$ Guieu et al. (2006)

$19=$ Guieu (2006)

$20=$ Hartigan \& Kenyon (2003)

$21=$ Hartigan et al. (1994)

$22=$ Hartmann (2002)

$23=$ Hartmann et al. (2005)

$24=$ Herbig et al. (1986)

$25=$ Itoh et al. (2005)

$26=$ Jensen \& Akeson (2003)

$27=$ Kenyon \& Hartmann (1995)

$28=$ Kenyon et al. (1993)

$29=$ Kenyon et al. (1998)

$30=$ Leinert \& Haas (1989)

$31=$ Leinert et al. (1993)

$32=$ Leinert et al. (1997)

$33=$ Luhman (2004)

$34=$ Luhman et al. (2003)

$35=$ Martín (2000)

36 = Martín \& Magazzù (1999)

$37=$ Martín et al. (1994)

$38=$ Martín et al. (1999)

$39=$ Martín et al. (2001)

$40=$ McCabe et al. (2006)

$41=$ Mohanty et al. (2005)

$42=$ Moneti \& Zinnecker (1991)

$43=$ Monin et al. (1998)

$44=$ Muzerolle et al. (1998)

$45=$ Muzerolle et al. (2003)

$46=$ Muzerolle et al. (2005)

$47=$ Prato \& Simon (1997)

$48=$ Rebull et al. (2004) and references therein

$49=$ Reipurth \& Zinnecker (1993)

$50=$ Richichi et al. (1994)

$51=$ Rodríguez et al. (1998)

$52=$ Simon et al. (1992) and references therein

$53=$ Simon et al. (1995)

$54=$ Smith et al. (2005)

$55=$ Strom \& Strom (1994)

$56=$ Walter et al. (1988)

$57=$ White \& Basri (2003)

$58=$ White \& Ghez (2001)

$59=$ White \& Hillenbrand (2004)

$60=$ Mundt et al. (1983)

$61=$ Welty $(1995)$

$62=$ Woitas $(2003)$

$63=$ Calvet et al. (2004)
$64=$ Reipurth et al. (2002)

$65=$ Walter et al. (2003) and references therein $66=$ Luhman (2006)

$67=$ Simon et al. (1996) 
M. Güdel et al.: XMM-Newton extended survey of Taurus, Online Material $p 3$

Table 3. Accepted good exposure time and fraction of total exposure time remaining after filtering out intervals of high background in the full energy band (0.5-7.3 keV). Calculated for each EPIC instrument in each XMM-Newton observation used in XEST. The penultimate column lists the approximate lowest detectable count-rate for a $4 \sigma$ detection in the PN; for a $30 \mathrm{ks}$ exposure in purely quiescent background, this would be $1.1 \mathrm{c} \mathrm{ks}^{-1}$. For XEST-26 only the MOS cameras were used and the MOS1 value has been multiplied by a factor 2.7 to calculate a PN-equivalent source count-rate. The final column lists the total number of detections made in the summed EPIC images.

\begin{tabular}{|c|c|c|c|c|c|c|c|c|}
\hline \multirow[t]{2}{*}{ Field } & \multicolumn{2}{|c|}{ M1 } & \multicolumn{2}{|c|}{ M2 } & \multicolumn{3}{|c|}{ PN } & \multirow{2}{*}{$\begin{array}{c}\text { EPIC } \\
N_{\text {det }}\end{array}$} \\
\hline & $\begin{array}{r}t_{\text {good }} \\
\mathrm{S}\end{array}$ & Per cent & $\begin{array}{r}t_{\text {good }} \\
\mathrm{S}\end{array}$ & Per cent & $\begin{array}{r}t_{\text {good }} \\
\mathrm{S}\end{array}$ & Per cent & $\begin{array}{c}C_{4 \sigma} \\
\mathrm{cks}^{-1}\end{array}$ & \\
\hline 01 & 50215 & 62.9 & 50944 & 63.9 & 41756 & 57.2 & 1.06 & 104 \\
\hline 02 & 39078 & 95.4 & 39638 & 96.8 & 27895 & 71.0 & 1.63 & 76 \\
\hline 03 & 22814 & 65.2 & 23908 & 68.3 & 15647 & 47.1 & 1.81 & 37 \\
\hline 04 & 29722 & 89.9 & 29710 & 89.9 & 29131 & 95.0 & 1.19 & 70 \\
\hline 05 & 24125 & 76.3 & 24229 & 76.6 & 15963 & 53.9 & 1.86 & 36 \\
\hline 06 & 30777 & 97.4 & 30894 & 97.7 & 27858 & 92.9 & 1.30 & 103 \\
\hline 07 & 30013 & 95.1 & 30183 & 95.6 & 25338 & 84.2 & 1.29 & 52 \\
\hline 08 & 35577 & 85.6 & 35841 & 86.2 & 26699 & 71.7 & 1.66 & 82 \\
\hline 09 & 25009 & 79.0 & 25170 & 79.5 & 19272 & 64.3 & 1.82 & 81 \\
\hline 10 & 28597 & 90.3 & 28649 & 90.5 & 25476 & 84.8 & 1.31 & 66 \\
\hline 11 & 34245 & 82.5 & 35075 & 84.2 & 30972 & 80.7 & 1.14 & 104 \\
\hline 12 & 30398 & 97.9 & 30451 & 98.1 & 28673 & 97.4 & 1.17 & 90 \\
\hline 13 & 24561 & 77.8 & 24979 & 79.1 & 20918 & 70.5 & 3.11 & 37 \\
\hline 14 & 28832 & 84.6 & 28942 & 84.9 & 24173 & 74.9 & 1.65 & 78 \\
\hline 15 & 23372 & 75.5 & 23475 & 75.9 & 19166 & 63.1 & 1.67 & 84 \\
\hline 16 & 23864 & 58.9 & 25683 & 63.3 & 13705 & 40.2 & 2.54 & 46 \\
\hline 17 & 26750 & 86.7 & 26804 & 86.9 & 22898 & 78.3 & 1.59 & 68 \\
\hline 18 & 28540 & 90.1 & 28654 & 90.5 & 26500 & 89.8 & 1.24 & 66 \\
\hline 19 & 30235 & 80.0 & 30544 & 80.8 & 26086 & 75.4 & 1.29 & 90 \\
\hline 20 & 31494 & 100.0 & 31479 & 100.0 & 29877 & 100.0 & 1.18 & 76 \\
\hline 21 & 29060 & 74.9 & 29094 & 75.4 & 18386 & 40.4 & 2.03 & 75 \\
\hline 22 & 55031 & 96.8 & 54931 & 96.6 & 50484 & 92.7 & 0.76 & 116 \\
\hline 23 & 57519 & 79.9 & 57496 & 79.8 & 41190 & 59.5 & 1.11 & 76 \\
\hline 24 & 32069 & 72.6 & 31991 & 72.4 & 24253 & 60.2 & 1.57 & 63 \\
\hline 25 & 16411 & 97.8 & 16466 & 98.1 & 14399 & 94.9 & 1.84 & 54 \\
\hline 26 & 124995 & 97.4 & 125270 & 97.6 & - & - & 0.81 & 165 \\
\hline 27 & 37770 & 87.3 & 27864 & 87.5 & 35416 & 85.2 & 1.16 & 153 \\
\hline 28 & 124833 & 95.4 & 124951 & 95.5 & 120693 & 93.1 & 0.52 & 199 \\
\hline
\end{tabular}


M. Güdel et al.: XMM-Newton extended survey of Taurus, Online Material p 4

Table 4. X-ray parameters of targets in XEST (1): Positions and count rates.

\begin{tabular}{|c|c|c|c|c|c|c|c|c|c|c|}
\hline XEST & Name & $\begin{array}{r}\mathrm{RA}_{\mathrm{X}} \\
\mathrm{h} \mathrm{m} \text { s }\end{array}$ & $\begin{array}{r}\operatorname{Dec}_{X} \\
\operatorname{deg} \prime^{\prime \prime \prime}\end{array}$ & $\begin{array}{r}\text { Poserr } \\
\left({ }^{\prime \prime}\right) \\
\end{array}$ & $\begin{array}{r}\text { Offset } \\
\left({ }^{\prime \prime}\right)\end{array}$ & $\mathrm{ML}_{\mathrm{det}}^{a}$ & Scts & $\begin{array}{r}T_{\exp } \\
(\mathrm{s}) \\
\end{array}$ & $\begin{array}{r}\text { Rate } \\
\left(\mathrm{ct} \mathrm{s}^{-1}\right)\end{array}$ & $\operatorname{Var}^{b}$ \\
\hline $27-115$ & HBC 352 & 35429.54 & 320302.2 & 1.04 & 0.89 & 9395 & 3832 & 15780 & 0.2429 & 0 \\
\hline $27-000$ & HBC 353 & 35430.17 & 320304.3 & 0.00 & 0.00 & 0 & $<183$ & 14469 & $<0.0127$ & 0 \\
\hline $06-005$ & HBC 358 AB & 40349.27 & 261053.1 & 1.48 & 1.22 & 1073 & 879 & 7371 & 0.1194 & 0 \\
\hline 06-007 & HBC 359 & 40350.82 & 261053.0 & 1.46 & 0.34 & 2921 & 1605 & 7668 & 0.2094 & 0 \\
\hline 06-059 & L1489 IRS & 40443.07 & 261856.3 & 1.46 & 0.10 & 7632 & 3336 & 46889 & 0.0712 & 0 \\
\hline $20-001$ & LkCa 1 & 41314.03 & 281909.9 & 1.58 & 1.71 & 455 & 336 & 7820 & 0.0430 & 0 \\
\hline $20-005$ & Anon 1 & 41327.28 & 281623.3 & 1.53 & 1.64 & 24658 & 8299 & 21632 & 0.3836 & 0 \\
\hline $20-000$ & IRAS $04108+2803$ A & 41353.29 & 281123.4 & 0.00 & 0.00 & 0 & $<26$ & 29957 & $<0.0009$ & 0 \\
\hline $20-022$ & IRAS $04108+2803 \mathrm{~B}$ & 41354.72 & 281132.2 & 1.55 & 0.70 & 1145 & 696 & 30670 & 0.0227 & 1 \\
\hline $20-000$ & 2M J04141188+28 & 41411.88 & 281153.5 & 0.00 & 0.00 & 0 & $<54$ & 48645 & $<0.0011$ & 0 \\
\hline $20-042$ & V773 Tau ABC & 41412.92 & 281211.8 & 1.53 & 0.60 & 213164 & 59321 & 48974 & 1.2113 & 0 \\
\hline $20-043$ & FM Tau & 41413.57 & 281248.6 & 1.54 & 0.61 & 2627 & 2988 & 48901 & 0.0611 & 0 \\
\hline $20-046$ & CW Tau & 41416.99 & 281100.6 & 1.84 & 2.80 & 11 & 52 & 35943 & 0.0015 & 0 \\
\hline $20-047$ & CIDA 1 & 41417.72 & 280609.8 & 1.69 & 1.46 & 63 & 105 & 33985 & 0.0031 & 0 \\
\hline $20-056$ & MHO 2/1 & 41426.41 & 280600.9 & 1.54 & 1.21 & 6260 & 2735 & 32359 & 0.0845 & 0 \\
\hline $20-058$ & MHO 3 & 41430.58 & 280514.7 & 1.55 & 0.40 & 1234 & 766 & 26856 & 0.0285 & 0 \\
\hline $20-069$ & FO Tau AB & 41449.23 & 281231.3 & 1.60 & 1.06 & 238 & 240 & 33352 & 0.0072 & 0 \\
\hline $20-073$ & CIDA 2 & 41505.16 & 280846.5 & 1.56 & 0.30 & 814 & 584 & 22342 & 0.0261 & 0 \\
\hline $23-002$ & CY Tau & 41733.61 & 282047.7 & 1.08 & 1.77 & 683 & 457 & 10256 & 0.0446 & 1 \\
\hline $24-002$ & CY Tau & 41733.73 & 282047.9 & 1.01 & 1.00 & 407 & 284 & 8366 & 0.0340 & 2 \\
\hline 23-004 & LkCa 5 & 41738.91 & 283259.6 & 1.04 & 0.98 & 5286 & 2855 & 32431 & 0.0880 & 0 \\
\hline 24-004 & LkCa 5 & 41738.94 & 283300.2 & 0.94 & 0.30 & 4767 & 2221 & 18626 & 0.1192 & 2 \\
\hline $23-008$ & CIDA 3 & 41749.67 & 282935.9 & 1.10 & 0.48 & 292 & 358 & 45960 & 0.0078 & 0 \\
\hline 24-008 & CIDA 3 & 41749.59 & 282935.3 & 0.99 & 1.28 & 338 & 332 & 26423 & 0.0126 & 0 \\
\hline 23-015 & V410 X3 & 41807.98 & 282602.8 & 1.09 & 0.94 & 576 & 561 & 64938 & 0.0086 & 0 \\
\hline 24-015 & V410 X3 & 41807.97 & 282602.1 & 0.99 & 1.61 & 388 & 341 & 37758 & 0.0090 & 0 \\
\hline 23-018 & V410 A13 & 41817.12 & 282840.5 & 1.45 & 1.41 & 20 & 72 & 66746 & 0.0011 & 0 \\
\hline $24-000$ & V410 A13 & 41817.11 & 282841.9 & 0.00 & 0.00 & 0 & $<24$ & 34966 & $<0.0007$ & 0 \\
\hline $23-000$ & V410 A24 & 41822.39 & 282437.6 & 0.00 & 0.00 & 0 & $<26$ & 68500 & $<0.0004$ & 0 \\
\hline $24-000$ & V410 A24 & 41822.39 & 282437.6 & 0.00 & 0.00 & 0 & $<30$ & 42210 & $<0.0007$ & 0 \\
\hline $23-029$ & V410 A25 & 41829.15 & 282618.9 & 1.04 & 0.69 & 4542 & 3586 & 76359 & 0.0470 & 0 \\
\hline $24-027$ & V410 A25 & 41829.12 & 282619.1 & 0.94 & 0.26 & 2447 & 1812 & 43216 & 0.0419 & 0 \\
\hline $23-032$ & V410 Tau ABC & 41831.08 & 282716.7 & 1.03 & 0.57 & 365212 & 101132 & 78334 & 1.2910 & 1 \\
\hline $24-028$ & V410 Tau ABC & 41831.09 & 282716.8 & 0.93 & 0.61 & 137848 & 38215 & 44779 & 0.8534 & 3 \\
\hline $23-033$ & DD Tau AB & 41831.11 & 281628.7 & 1.08 & 0.40 & 1006 & 885 & 39614 & 0.0223 & 1 \\
\hline $24-029$ & DD Tau AB & 41831.10 & 281629.3 & 1.04 & 0.50 & 295 & 320 & 22841 & 0.0140 & 0 \\
\hline $23-035$ & CZ Tau AB & 41831.60 & 281659.2 & 1.05 & 0.71 & 1756 & 1271 & 41620 & 0.0305 & 0 \\
\hline $24-030$ & CZ Tau AB & 41831.57 & 281659.2 & 0.97 & 0.75 & 1509 & 995 & 23898 & 0.0417 & 0 \\
\hline $23-036$ & IRAS $04154+2823$ & 41831.94 & 283115.6 & 1.11 & 1.20 & 211 & 308 & 64773 & 0.0048 & 1 \\
\hline $24-031$ & IRAS $04154+2823$ & 41831.88 & 283114.9 & 1.59 & 2.04 & 18 & 61 & 36863 & 0.0017 & 0 \\
\hline $23-037$ & V410 X2 & 41834.40 & 283030.1 & 1.04 & 0.67 & 5641 & 3007 & 65004 & 0.0463 & 0 \\
\hline $24-032$ & V410 X2 & 41834.38 & 283030.6 & 0.94 & 1.01 & 3959 & 2015 & 36402 & 0.0554 & 1 \\
\hline $23-045$ & V410 X4 & 41840.24 & 282425.1 & 1.06 & 0.61 & 859 & 760 & 69092 & 0.0110 & 0 \\
\hline $24-038$ & V410 X4 & 41840.26 & 282425.2 & 0.95 & 0.80 & 1153 & 778 & 39074 & 0.0199 & 2 \\
\hline $23-047$ & V892 Tau & 41840.64 & 281915.9 & 1.03 & 0.48 & 58194 & 20108 & 48862 & 0.4115 & 0 \\
\hline $24-040$ & V892 Tau & 41840.62 & 281915.6 & 0.93 & 0.10 & 111462 & 31125 & 28058 & 1.1093 & 1 \\
\hline $23-048$ & LR 1 & 41841.29 & 282726.9 & 1.22 & 1.97 & 45 & 134 & 69240 & 0.0019 & 1 \\
\hline $24-000$ & LR 1 & 41841.33 & 282725.0 & 0.00 & 0.00 & 0 & $<67$ & 41278 & $<0.0016$ & 0 \\
\hline $23-050$ & V410 X7 & 41842.53 & 281849.9 & 1.04 & 0.41 & 2808 & 2319 & 46487 & 0.0499 & 1 \\
\hline $24-042$ & V410 X7 & 41842.49 & 281850.0 & 0.93 & 0.24 & 10724 & 6309 & 26634 & 0.2369 & 1 \\
\hline $23-000$ & V410 A20 & 41845.06 & 282052.8 & 0.00 & 0.00 & 0 & $<23$ & 32774 & $<0.0007$ & 0 \\
\hline $24-000$ & V410 A20 & 41845.06 & 282052.8 & 0.00 & 0.00 & 0 & $<15$ & 17241 & $<0.0009$ & 0 \\
\hline $23-056$ & Hubble 4 & 41847.03 & 282007.7 & 1.03 & 0.42 & 95259 & 27648 & 45560 & 0.6069 & 0 \\
\hline $24-047$ & Hubble 4 & 41847.02 & 282007.7 & 0.93 & 0.48 & 43119 & 14030 & 26926 & 0.5211 & 0 \\
\hline $23-000$ & KPNO-Tau 2 & 41851.16 & 281433.2 & 0.00 & 0.00 & 0 & $<28$ & 15428 & $<0.0018$ & 0 \\
\hline $24-000$ & KPNO-Tau 2 & 41851.16 & 281433.2 & 0.00 & 0.00 & 0 & $<15$ & 8642 & $<0.0018$ & 0 \\
\hline $23-000$ & CoKu Tau 1 & 41851.48 & 282026.5 & 0.00 & 0.00 & 0 & $<128$ & 48084 & $<0.0027$ & 0 \\
\hline $24-000$ & CoKu Tau 1 & 41851.48 & 282026.5 & 0.00 & 0.00 & 0 & $<57$ & 27535 & $<0.0021$ & 0 \\
\hline $23-061$ & V410 X6 & 41901.07 & 281942.1 & 1.10 & 0.54 & 298 & 369 & 38775 & 0.0095 & 0 \\
\hline
\end{tabular}


Table 4. continued.

\begin{tabular}{|c|c|c|c|c|c|c|c|c|c|c|}
\hline XEST & Name & $\begin{array}{r}\mathrm{RA}_{\mathrm{X}} \\
\mathrm{h} \mathrm{m} \text { s }\end{array}$ & $\begin{array}{r}\operatorname{Dec}_{X} \\
\operatorname{deg} \prime^{\prime \prime \prime}\end{array}$ & $\begin{array}{r}\text { Poserr } \\
\left({ }^{\prime \prime}\right)\end{array}$ & $\begin{array}{r}\text { Offset } \\
\left({ }^{\prime \prime}\right)\end{array}$ & $\mathrm{ML}_{\mathrm{det}}^{a}$ & Scts & $\begin{array}{r}T_{\exp } \\
(\mathrm{s})\end{array}$ & $\begin{array}{r}\text { Rate } \\
\left(\mathrm{ct} \mathrm{s}^{-1}\right)\end{array}$ & $\overline{\operatorname{Var}^{b}}$ \\
\hline $24-054$ & V410 X6 & 41901.08 & 281942.2 & 0.98 & 0.44 & 514 & 442 & 22047 & 0.0201 & 2 \\
\hline $23-063$ & V410 X5 & 41901.94 & 282234.4 & 1.05 & 1.31 & 1688 & 1179 & 43645 & 0.0270 & 0 \\
\hline $24-055$ & V410 X5 & 41901.94 & 282233.8 & 0.95 & 0.80 & 2422 & 1339 & 24869 & 0.0539 & 2 \\
\hline $23-067$ & FQ Tau AB & 41912.77 & 282933.3 & 1.21 & 0.56 & 100 & 178 & 34347 & 0.0052 & 0 \\
\hline $24-058$ & FQ Tau AB & 41912.77 & 282934.3 & 1.20 & 1.31 & 64 & 101 & 18919 & 0.0054 & 0 \\
\hline $28-100$ & BP Tau & 41915.90 & 290626.6 & 2.24 & 0.84 & 142902 & 46278 & 197539 & 0.2343 & 1 \\
\hline 23-074 & V819 Tau AB & 41926.26 & 282614.5 & 1.04 & 0.20 & 19437 & 7785 & 28888 & 0.2695 & 0 \\
\hline $24-061$ & V819 Tau AB & 41926.26 & 282614.7 & 0.94 & 0.40 & 9399 & 3967 & 16434 & 0.2414 & 0 \\
\hline $16-000$ & IRAS 04166+2706 & 41943.00 & 271333.7 & 0.00 & 0.00 & 0 & $<30$ & 30078 & $<0.0010$ & 0 \\
\hline $16-000$ & IRAS $04169+2702$ & 41958.45 & 270957.1 & 0.00 & 0.00 & 0 & $<38$ & 23883 & $<0.0016$ & 0 \\
\hline $11-000$ & CFHT-Tau 19 & 42107.95 & 270220.4 & 0.00 & 0.00 & 0 & $<18$ & 22031 & $<0.0008$ & 0 \\
\hline $11-000$ & IRAS 04181+2655 & 42110.90 & 270206.0 & 0.00 & 0.00 & 0 & $<18$ & 22755 & $<0.0008$ & 0 \\
\hline $11-000$ & IRAS $04181+2654 \mathrm{AB}$ & 42111.47 & 270109.4 & 0.00 & 0.00 & 0 & $<34$ & 24662 & $<0.0014$ & 0 \\
\hline $11-023$ & 2M J04213459 & 42134.51 & 270138.7 & 1.88 & 1.22 & 140 & 171 & 38717 & 0.0044 & 0 \\
\hline $01-028$ & IRAS 04187+1927 & 42143.23 & 193412.5 & 1.55 & 0.81 & 2842 & 1460 & 30378 & 0.0481 & 0 \\
\hline $11-037$ & CFHT-Tau 10 & 42146.39 & 265928.4 & 2.25 & 1.61 & 9 & 35 & 48347 & 0.0007 & 0 \\
\hline $11-000$ & 2 M J04215450+2652 & 42154.51 & 265231.5 & 0.00 & 0.00 & 0 & $<17$ & 37210 & $<0.0004$ & 0 \\
\hline $21-038$ & RY Tau & 42157.40 & 282635.4 & 1.36 & 0.10 & 17357 & 6495 & 23482 & 0.2766 & 0 \\
\hline $21-039$ & HD 283572 & 42158.87 & 281806.1 & 1.36 & 0.64 & 282825 & 76762 & 37198 & 2.0636 & 0 \\
\hline 01-045 & T Tau N(+Sab) & 42159.44 & 193205.8 & 1.54 & 0.62 & 211562 & 59050 & 72108 & 0.8189 & 0 \\
\hline $11-054$ & Haro 6-5 B & 42200.72 & 265732.4 & 1.88 & 0.29 & 28 & 188 & 51812 & 0.0036 & 0 \\
\hline $11-057$ & FS Tau AC & 42202.19 & 265730.9 & 1.78 & 0.42 & 20767 & 7398 & 51050 & 0.1449 & 3 \\
\hline $21-044$ & LkCa 21 & 42203.12 & 282539.1 & 1.36 & 0.28 & 6256 & 2932 & 25588 & 0.1146 & 0 \\
\hline $01-054$ & RX J0422.1+1934 & 42204.91 & 193448.8 & 1.54 & 0.87 & 54106 & 17801 & 52504 & 0.3390 & 0 \\
\hline $01-062$ & 2M J04221332+1934 & 42213.24 & 193440.2 & 1.72 & 1.51 & 69 & 117 & 33836 & 0.0035 & 0 \\
\hline $11-079$ & CFHT-Tau 21 & 42216.80 & 265458.2 & 1.82 & 1.22 & 286 & 274 & 39533 & 0.0070 & 0 \\
\hline 02-013 & FV Tau AB & 42653.48 & 260654.9 & 1.60 & 0.84 & 781 & 662 & 31329 & 0.0212 & 0 \\
\hline $02-000$ & FV Tau/c AB & 42654.41 & 260651.0 & 0.00 & 0.00 & 0 & $<94$ & 35140 & $<0.0027$ & 0 \\
\hline 02-016 & KPNO-Tau 13 & 42657.34 & 260627.2 & 1.63 & 1.21 & 217 & 288 & 22911 & 0.0126 & 0 \\
\hline 02-000 & DG Tau B & 42702.66 & 260530.5 & 0.00 & 0.00 & 0 & $<27$ & 38087 & $<0.0007$ & 0 \\
\hline $02-022$ & DG Tau A & 42704.70 & 260615.5 & 1.59 & 0.80 & 1669 & 1185 & 40865 & 0.0290 & 2 \\
\hline 02-000 & KPNO-Tau 4 & 42728.00 & 261205.3 & 0.00 & 0.00 & 0 & $<41$ & 50463 & $<0.0008$ & 0 \\
\hline $02-000$ & IRAS $04248+2612 \mathrm{AB}$ & 42757.31 & 261918.3 & 0.00 & 0.00 & 0 & $<25$ & 17943 & $<0.0014$ & 0 \\
\hline $15-020$ & JH 507 & 42920.67 & 263340.2 & 1.56 & 0.73 & 2000 & 1102 & 28851 & 0.0382 & 0 \\
\hline 13-004 & GV Tau AB & 42923.69 & 243300.2 & 1.63 & 0.55 & 518 & 615 & 20976 & 0.0293 & 1 \\
\hline $13-000$ & IRAS 04264+2433 & 42930.08 & 243955.1 & 0.00 & 0.00 & 0 & $<68$ & 26868 & $<0.0025$ & 0 \\
\hline $15-040$ & DH Tau AB & 42941.56 & 263258.5 & 1.55 & 0.20 & 130666 & 36137 & 33680 & 1.0729 & 3 \\
\hline $15-042$ & DI Tau AB & 42942.51 & 263249.3 & 1.56 & 0.40 & 926 & 3305 & 33773 & 0.0979 & 0 \\
\hline $15-044$ & KPNO-Tau 5 & 42945.81 & 263047.9 & 1.82 & 2.06 & 40 & 86 & 32576 & 0.0026 & 0 \\
\hline $14-006$ & IQ Tau A & 42951.56 & 260644.9 & 2.02 & 0.00 & 3811 & 1972 & 22748 & 0.0867 & 1 \\
\hline $13-000$ & CFHT-Tau 20 & 42959.51 & 243307.9 & 0.00 & 0.00 & 0 & $<22$ & 30935 & $<0.0007$ & 0 \\
\hline $14-000$ & KPNO-Tau 6 & 43007.24 & 260820.8 & 0.00 & 0.00 & 0 & $<27$ & 26206 & $<0.0010$ & 0 \\
\hline $13-035$ & FX Tau AB & 43029.59 & 242647.2 & 1.65 & 2.22 & 313 & 378 & 7123 & 0.0532 & 0 \\
\hline $14-057$ & DK Tau AB & 43044.25 & 260125.5 & 2.01 & 1.00 & 8459 & 3697 & 37386 & 0.0989 & 0 \\
\hline $14-000$ & KPNO-Tau 7 & 43057.19 & 255639.5 & 0.00 & 0.00 & 0 & $<31$ & 25489 & $<0.0012$ & 0 \\
\hline $22-013$ & MHO 9 & 43115.83 & 182005.9 & 1.62 & 1.48 & 777 & 611 & 37684 & 0.0162 & 0 \\
\hline $22-021$ & MHO 4 & 43124.18 & 180021.6 & 1.63 & 1.71 & 700 & 569 & 37887 & 0.0150 & 0 \\
\hline $22-040$ & L1551 IRS5 & 43134.02 & 180804.2 & 1.68 & 1.11 & 139 & 191 & 74201 & 0.0026 & 0 \\
\hline $22-042$ & LkHa 358 & 43136.13 & 181343.3 & 1.66 & 0.00 & 115 & 273 & 76104 & 0.0036 & 0 \\
\hline $22-000$ & HH 30 & 43137.47 & 181224.5 & 0.00 & 0.00 & 0 & $<21$ & 81198 & $<0.0003$ & 0 \\
\hline $22-043$ & HL Tau & 43138.38 & 181357.1 & 1.60 & 1.04 & 6052 & 5618 & 75594 & 0.0743 & 0 \\
\hline $22-047$ & XZ Tau AB & 43140.05 & 181356.6 & 1.60 & 0.66 & 60086 & 18391 & 75388 & 0.2440 & 1 \\
\hline $22-056$ & L1551 NE & 43144.53 & 180832.3 & 1.98 & 1.39 & 13 & 47 & 80720 & 0.0006 & 0 \\
\hline 03-005 & HK Tau AB & 43150.57 & 242416.1 & 1.77 & 2.00 & 60 & 86 & 19411 & 0.0045 & 0 \\
\hline $22-070$ & V710 Tau BA & 43157.72 & 182137.2 & 1.60 & 1.34 & 11966 & 4639 & 35159 & 0.1320 & 0 \\
\hline $19-009$ & JH 665 & 43158.50 & 254330.6 & 2.27 & 1.07 & 108 & 123 & 8791 & 0.0141 & 0 \\
\hline $22-089$ & L1551 51 & 43209.45 & 175722.9 & 1.60 & 2.57 & 11539 & 4287 & 13801 & 0.3107 & 2 \\
\hline 22-097 & V827 Tau & 43214.50 & 182014.5 & 1.60 & 1.02 & 36001 & 10530 & 18303 & 0.5753 & 0 \\
\hline 03-016 & Haro 6-13 & 43215.40 & 242859.9 & 1.59 & 0.24 & 338 & 288 & 20435 & 0.0141 & 0 \\
\hline
\end{tabular}


Table 4. continued.

\begin{tabular}{|c|c|c|c|c|c|c|c|c|c|c|}
\hline XEST & Name & $\begin{array}{l}R^{2} A_{X} \\
\text { h }{ }^{2} \text { s }\end{array}$ & $\begin{array}{r}\operatorname{Dec}_{X} \\
\operatorname{deg} \prime^{\prime} \prime \prime\end{array}$ & $\begin{array}{r}\text { Poserr } \\
\left({ }^{\prime \prime}\right)\end{array}$ & $\begin{array}{r}\text { Offset } \\
\left({ }^{\prime \prime}\right)\end{array}$ & $\mathrm{ML}_{\mathrm{det}}^{a}$ & Scts & $\begin{array}{r}T_{\exp } \\
(\mathrm{s})\end{array}$ & $\begin{array}{r}\text { Rate } \\
\left(\mathrm{ct} \mathrm{s}^{-1}\right)\end{array}$ & $\overline{\operatorname{Var}^{b}}$ \\
\hline $22-100$ & V826 Tau & 43215.91 & 180139.1 & 1.60 & 1.08 & 94853 & 27095 & 36121 & 0.7501 & $\overline{0}$ \\
\hline $22-101$ & MHO 5 & 43216.07 & 181246.5 & 1.62 & 0.10 & 1457 & 963 & 51887 & 0.0186 & 0 \\
\hline 03-017 & CFHT-Tau 7 & 43217.83 & 242213.1 & 1.71 & 1.94 & 29 & 121 & 30227 & 0.0040 & 0 \\
\hline 03-019 & V928 Tau AB & 43218.90 & 242226.1 & 1.54 & 1.14 & 4972 & 2243 & 30636 & 0.0732 & 0 \\
\hline $03-022$ & FY Tau & 43230.68 & 241958.1 & 1.55 & 1.58 & 4931 & 2110 & 25663 & 0.0822 & 0 \\
\hline 03-023 & FZ Tau & 43231.83 & 242004.1 & 1.59 & 1.46 & 213 & 440 & 19003 & 0.0232 & 0 \\
\hline $17-002$ & IRAS $04295+2251$ & 43232.07 & 225726.4 & 1.36 & 0.41 & 322 & 303 & 17292 & 0.0176 & 2 \\
\hline 19-049 & UZ Tau E+W(AB) & 43242.89 & 255232.6 & 2.19 & 2.52 & 4110 & 2085 & 44868 & 0.0465 & 0 \\
\hline $17-009$ & JH 112 & 43249.09 & 225301.9 & 1.33 & 0.94 & 1517 & 867 & 24276 & 0.0357 & 0 \\
\hline $03-031$ & CFHT-Tau 5 & 43250.28 & 242211.4 & 1.73 & 0.24 & 54 & 82 & 15890 & 0.0052 & 0 \\
\hline $04-003$ & CFHT-Tau 5 & 43250.31 & 242211.1 & 1.73 & 0.74 & 367 & 312 & 26369 & 0.0119 & 0 \\
\hline 03-035 & MHO 8 & 43301.97 & 242102.7 & 1.66 & 2.70 & 105 & 130 & 17386 & 0.0075 & 0 \\
\hline 04-009 & MHO 8 & 43302.02 & 242059.0 & 1.77 & 1.14 & 181 & 172 & 28001 & 0.0062 & 0 \\
\hline 04-010 & GH Tau AB & 43306.33 & 240932.5 & 1.78 & 2.13 & 96 & 169 & 11180 & 0.0151 & 0 \\
\hline 04-012 & V807 Tau SNab & 43306.75 & 240954.6 & 1.68 & 1.56 & 10380 & 4068 & 20068 & 0.2027 & 0 \\
\hline $18-004$ & KPNO-Tau 14 & 43307.79 & 261605.7 & 2.13 & 0.94 & 1424 & 840 & 19063 & 0.0441 & 0 \\
\hline $17-000$ & CFHT-Tau 12 & 43309.46 & 224648.7 & 0.00 & 0.00 & 0 & $<10$ & 30963 & $<0.0003$ & 0 \\
\hline 04-016 & V830 Tau & 43309.98 & 243343.4 & 1.68 & 0.68 & 44530 & 13508 & 15863 & 0.8516 & 3 \\
\hline $18-000$ & IRAS S04301+261 & 43314.36 & 261423.5 & 0.00 & 0.00 & 0 & $<17$ & 22545 & $<0.0008$ & 0 \\
\hline $17-000$ & IRAS $04302+2247$ & 43316.50 & 225320.4 & 0.00 & 0.00 & 0 & $<28$ & 29406 & $<0.0010$ & 0 \\
\hline $17-027$ & IRAS $04303+2240$ & 43319.10 & 224634.1 & 1.31 & 0.43 & 9238 & 3723 & 30569 & 0.1218 & 0 \\
\hline 04-034 & GI Tau & 43334.06 & 242117.9 & 1.69 & 0.90 & 792 & 1707 & 46957 & 0.0364 & 0 \\
\hline $04-035$ & GK Tau AB & 43334.52 & 242108.0 & 1.68 & 2.17 & 17201 & 6635 & 47269 & 0.1404 & 0 \\
\hline $18-019$ & IS Tau AB & 43336.83 & 260948.3 & 2.13 & 1.05 & 3999 & 1835 & 31294 & 0.0586 & 0 \\
\hline $17-058$ & CI Tau & 43352.02 & 225030.8 & 1.34 & 0.66 & 647 & 485 & 23775 & 0.0204 & 1 \\
\hline $18-030$ & IT Tau AB & 43354.72 & 261326.7 & 2.12 & 0.84 & 58042 & 17849 & 44176 & 0.4041 & 0 \\
\hline $17-066$ & JH 108 & 43411.01 & 225144.7 & 1.32 & 0.34 & 9235 & 3875 & 14379 & 0.2695 & 1 \\
\hline $17-068$ & CFHT-BD Tau 1 & 43415.31 & 225033.1 & 1.45 & 2.17 & 163 & 204 & 14010 & 0.0146 & 2 \\
\hline $25-026$ & AA Tau & 43455.39 & 242853.6 & 1.82 & 0.57 & 3334 & 1471 & 24628 & 0.0597 & 0 \\
\hline 09-010 & HO Tau AB & 43520.31 & 223211.1 & 1.58 & 3.82 & 82 & 122 & 18311 & 0.0067 & 0 \\
\hline 08-019 & FF Tau AB & 43520.90 & 225424.3 & 1.60 & 0.10 & 3812 & 2076 & 30501 & 0.0681 & 0 \\
\hline $12-040$ & DN Tau & 43527.34 & 241458.9 & 1.57 & 0.41 & 28146 & 9246 & 47293 & 0.1955 & 0 \\
\hline $12-000$ & IRAS $04325+2402 \mathrm{AB}$ & 43535.39 & 240819.4 & 0.00 & 0.00 & 0 & $<44$ & 34141 & $<0.0013$ & 0 \\
\hline $12-059$ & CoKu Tau 3 AB & 43540.95 & 241109.0 & 1.57 & 0.24 & 69838 & 20015 & 38317 & 0.5224 & 0 \\
\hline 09-022 & KPNO-Tau 8 & 43541.91 & 223410.9 & 1.44 & 1.20 & 3919 & 1858 & 27822 & 0.0668 & 0 \\
\hline 08-037 & HQ Tau AB & 43547.39 & 225022.0 & 1.59 & 0.75 & 25465 & 9258 & 30355 & 0.3050 & 0 \\
\hline 09-026 & HQ Tau AB & 43547.33 & 225020.9 & 1.43 & 0.81 & 20604 & 7171 & 14030 & 0.5111 & 3 \\
\hline $08-043$ & KPNO-Tau 15 & 43551.13 & 225240.1 & 1.59 & 0.41 & 25254 & 10329 & 46282 & 0.2232 & 0 \\
\hline 09-031 & KPNO-Tau 15 & 43551.01 & 225240.3 & 1.49 & 1.26 & 633 & 529 & 11974 & 0.0443 & 0 \\
\hline $08-000$ & KPNO-Tau 9 & 43551.43 & 224911.9 & 0.00 & 0.00 & 0 & $<28$ & 37232 & $<0.0008$ & 0 \\
\hline 09-000 & KPNO-Tau 9 & 43551.43 & 224911.9 & 0.00 & 0.00 & 0 & $<26$ & 17141 & $<0.0016$ & 0 \\
\hline 08-048 & HP Tau AB & 43552.78 & 225422.9 & 1.59 & 0.20 & 3573 & 5706 & 49366 & 0.1156 & 0 \\
\hline 08-051a & HP Tau/G3 AB & 43553.50 & 225409.0 & 0.00 & 0.00 & 134269 & 3006 & 48905 & 0.0648 & 0 \\
\hline $08-051$ & HP Tau/G2 & 43554.14 & 225412.8 & 1.59 & 0.71 & 134269 & 39787 & 48905 & 0.8136 & 0 \\
\hline 08-058 & Haro 6-28 AB & 43556.80 & 225437.1 & 1.63 & 1.23 & 290 & 614 & 49680 & 0.0124 & 0 \\
\hline $08-000$ & CFHT-BD Tau 2 & 43610.39 & 225956.0 & 0.00 & 0.00 & 0 & $<29$ & 36650 & $<0.0008$ & 0 \\
\hline $08-080$ & CFHT-BD Tau 3 & 43638.91 & 225813.2 & 1.86 & 1.36 & 20 & 64 & 25098 & 0.0026 & 0 \\
\hline $05-005$ & CFHT-Tau 6 & 43904.10 & 254426.4 & 1.90 & 1.89 & 48 & 84 & 21111 & 0.0040 & 0 \\
\hline $05-000$ & IRAS $04361+2547$ & 43913.89 & 255320.9 & 0.00 & 0.00 & 0 & $<35$ & 11917 & $<0.0030$ & 0 \\
\hline $05-013$ & GN Tau AB & 43920.86 & 254500.9 & 1.77 & 1.38 & 301 & 248 & 22952 & 0.0108 & 0 \\
\hline 05-017 & IRAS $04365+2535$ & 43935.25 & 254145.4 & 1.93 & 1.07 & 34 & 75 & 31546 & 0.0024 & 0 \\
\hline $05-024$ & IRAS $04369+2539$ & 43955.70 & 254501.7 & 1.75 & 0.74 & 435 & 335 & 20227 & 0.0166 & 0 \\
\hline 07-011 & JH 223 & 44049.54 & 255120.0 & 1.87 & 0.90 & 374 & 318 & 24335 & 0.0131 & 0 \\
\hline $07-022$ & Haro 6-32 & 44104.32 & 255755.5 & 1.89 & 1.23 & 351 & 311 & 17222 & 0.0181 & 0 \\
\hline $07-000$ & ITG 33 A & 44108.26 & 255607.5 & 0.00 & 0.00 & 0 & $<21$ & 22254 & $<0.0010$ & 0 \\
\hline $07-000$ & CFHT-Tau 8 & 44110.78 & 255511.7 & 0.00 & 0.00 & 0 & $<16$ & 23759 & $<0.0007$ & 0 \\
\hline $07-000$ & IRAS $04381+2540$ & 44112.68 & 254635.4 & 0.00 & 0.00 & 0 & $<32$ & 44259 & $<0.0007$ & 0 \\
\hline 07-041 & IRAS $04385+2550 \mathrm{AB}$ & 44138.80 & 255627.4 & 1.86 & 0.66 & 503 & 397 & 16994 & 0.0234 & 2 \\
\hline $10-017$ & CoKuLk332/G2 AB & 44205.47 & 252256.2 & 1.68 & 0.29 & 17061 & 6651 & 36423 & 0.1826 & 0 \\
\hline
\end{tabular}


Table 4. continued.

\begin{tabular}{|c|c|c|c|c|c|c|c|c|c|c|}
\hline XEST & Name & 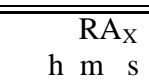 & $\begin{array}{r}\operatorname{Dec}_{X} \\
\operatorname{deg} \prime^{\prime \prime \prime}\end{array}$ & $\begin{array}{r}\text { Poserr } \\
\left({ }^{\prime \prime}\right)\end{array}$ & $\begin{array}{r}\text { Offset } \\
\left({ }^{\prime \prime}\right)\end{array}$ & $\mathrm{ML}_{\mathrm{det}}^{a}$ & Scts & $\begin{array}{r}T_{\exp } \\
(\mathrm{s})\end{array}$ & $\begin{array}{r}\text { Rate } \\
\left(\text { ct s }^{-1}\right)\end{array}$ & $\overline{\operatorname{Var}^{b}}$ \\
\hline 10-018 & CoKuLk332/G1 AB & 44207.32 & 252303.2 & 1.69 & 0.14 & 243 & 1448 & 30835 & 0.0470 & $\overline{0}$ \\
\hline $10-020$ & V955 Tau AB & 44207.76 & 252311.6 & 1.70 & 0.24 & 308 & 1360 & 23616 & 0.0576 & 0 \\
\hline $10-034$ & CIDA 7 & 44221.02 & 252035.5 & 1.83 & 1.10 & 71 & 119 & 43153 & 0.0028 & 2 \\
\hline $10-045$ & DP Tau & 44237.72 & 251536.8 & 1.83 & 0.75 & 64 & 93 & 30465 & 0.0031 & 2 \\
\hline $10-060$ & GO Tau & 44303.12 & 252019.8 & 1.72 & 1.08 & 611 & 479 & 21286 & 0.0225 & 0 \\
\hline $26-012$ & $2 \mathrm{M} \mathrm{J} 04552333+30$ & 45523.12 & 302738.2 & 1.98 & 3.15 & 17 & 54 & 28154 & 0.0019 & 0 \\
\hline $26-034$ & $2 \mathrm{M} \mathrm{J} 04554046+30$ & 45540.34 & 303907.1 & 2.19 & 2.09 & 4 & 45 & 35843 & 0.0013 & 0 \\
\hline $26-043$ & AB Aur & 45545.83 & 303303.3 & 1.73 & 1.10 & 7917 & 3472 & 69171 & 0.0502 & 0 \\
\hline $26-050$ & 2MJ04554757/801 & 45547.83 & 302805.3 & 1.76 & 4.13 & 426 & 418 & 43514 & 0.0096 & 2 \\
\hline $26-067$ & SU Aur & 45559.34 & 303400.9 & 1.73 & 0.87 & 245679 & 59141 & 78106 & 0.7572 & 1 \\
\hline $26-072$ & HBC 427 & 45602.05 & 302103.9 & 1.73 & 0.40 & 91805 & 25437 & 28045 & 0.9070 & 1 \\
\hline \multicolumn{6}{|c|}{ Additional sources from Chandra } & Sig. ${ }^{a}$ & & & & \\
\hline C1-0 & KPNO-Tau 10 & 41749.55 & 281331.9 & 0.00 & 0.00 & 0.0 & 0 & 17734 & 0.0000 & $\overline{0}$ \\
\hline C1-1 & IRAS $04158+2805$ & 41858.15 & 281223.3 & 0.93 & 0.24 & 6.0 & 100 & 17734 & 0.0056 & 0 \\
\hline C2-1 & Haro 6-5 B & 42200.71 & 265732.2 & 0.07 & 0.33 & 15.4 & 32 & 29674 & 0.0011 & 0 \\
\hline $\mathrm{C} 2-2$ & FS Tau AC & 42202.20 & 265730.4 & 0.04 & 0.29 & 101.0 & 254 & 29674 & 0.0086 & 0 \\
\hline C3-1 & FV Tau/c AB & 42654.34 & 260651.3 & 0.21 & 0.99 & 6.2 & 13 & 29717 & 0.0005 & 0 \\
\hline C3-2 & DG Tau B & 42702.58 & 260530.8 & 0.22 & 1.12 & 4.3 & 9 & 29717 & 0.0003 & 0 \\
\hline C4-1 & GV Tau AB & 42923.74 & 243300.5 & 0.05 & 0.24 & 26.7 & 57 & 24650 & 0.0023 & 0 \\
\hline C5-2 & HN Tau AB & 43339.34 & 175151.3 & 1.40 & 1.11 & 3.6 & 22 & 4679 & 0.0047 & 0 \\
\hline C5-1 & L1551 55 & 43243.70 & 180254.4 & 1.17 & 1.95 & 6.7 & 74 & 4679 & 0.0160 & 0 \\
\hline C5-4 & HD 28867 & 43333.06 & 180100.1 & 0.01 & 1.18 & 39.2 & 1582 & 4679 & 0.3390 & 0 \\
\hline C5-3 & DM Tau & 43348.63 & 181011.5 & 1.28 & 1.97 & 5.1 & 33 & 4679 & 0.0071 & 0 \\
\hline C6-1 & CFHT-BD Tau 4 & 43947.50 & 260140.8 & 0.14 & 0.27 & 16.8 & 30 & 19317 & 0.0016 & 0 \\
\hline C6-0 & L1527 IRS & 43953.59 & 260305.5 & 0.00 & 0.00 & 0.0 & 0 & 19317 & 0.0000 & 0 \\
\hline C6-0 & CFHT-Tau 17 & 44001.75 & 255629.2 & 0.00 & 0.00 & 0.0 & 0 & 19317 & 0.0000 & 0 \\
\hline C6-2 & IRAS $04370+2559$ & 44008.02 & 260525.5 & 0.09 & 0.29 & 74.6 & 229 & 19317 & 0.0119 & 0 \\
\hline
\end{tabular}

${ }^{a}$ Maximum likelihood for detection for XMM-Newton data, CIAO WAVDETECT "Significance" for Chandra data.

${ }^{b}$ Variability flag: $0=$ no or only low-level variability; 1 = clear flaring, flare intervals removed in spectral fit; $2=$ clear flaring observed but flare intervals not removed; 3 = slow decay of flare throughout observation, all data used.

Notes on individual objects:

- CoKu Tau 1 = XEST-23-000: Chandra observation C1 may contain a marginal off-axis detection ( $\approx 6$ counts).

- V892 Tau= XEST-23-047 = XEST-24-040: Companion at 4" is visible as a faint source in Chandra observation C1.

- HP Tau/G2 and G3 = XEST-08-051 (separation: 10") were treated as one source. The ratios of the counts and rates were derived from PSF fitting in the image. 
M. Güdel et al.: XMM-Newton extended survey of Taurus, Online Material $p 8$

Table 5. X-ray parameters of targets in XEST (2): Plasma parameters from the DEM fits.

\begin{tabular}{|c|c|c|c|c|c|c|c|c|c|c|c|}
\hline XEST & Name & $\begin{array}{l}N_{\mathrm{H}}(1 \sigma \text { range }) \\
\left(10^{22} \mathrm{~cm}^{-2}\right)\end{array}$ & $\begin{array}{l}T_{0} \\
\text { (MK }\end{array}$ & $(1 \sigma$ range $)$ & $(1 \sigma$ range $)$ & $\begin{array}{r}\mathrm{EM}_{t}^{a} \\
\left(10^{52}\right)\end{array}$ & $\begin{array}{l}L_{\mathrm{X}}^{b} \quad \text { (range) } \\
\left(10^{30} \mathrm{erg} \mathrm{s}^{-1}\right)\end{array}$ & $\begin{array}{r}\log \\
L_{\mathrm{X}} / L_{*}\end{array}$ & $\begin{array}{r}T_{\text {av }} \\
(\mathrm{MK})\end{array}$ & $\chi_{\text {red }}^{2}$ & d.o.f \\
\hline $27-115$ & HBC 352 & $0.22(0.19,0.25)$ & 6.5 & $(5.0,8.2)$ & $-0.60(-0.79,-0.47)$ & 25.21 & $2.657(2.43,2.87)$ & -3.03 & 12.00 & 0.85 & 129 \\
\hline $27-000$ & HBC 353 & 0.17 & 10.0 & & -1.00 & & $<0.176$ & $<-4.04$ & & & $\ldots$ \\
\hline 06-005 & HBC $358 \mathrm{AB}$ & $0.01(0.00,0.04)$ & 4.7 & $(3.0,7.5)$ & $-0.60(-1.24,-0.34)$ & 3.79 & $0.383(0.37,0.44)$ & -3.45 & 9.42 & 0.74 & 21 \\
\hline 06-007 & HBC 359 & $0.01(0.00,0.02)$ & 7.2 & $(5.8,8.9)$ & $-1.25(-1.84,-0.91)$ & 6.65 & $0.663(0.64,0.69)$ & -3.18 & 9.20 & 1.05 & 57 \\
\hline 06-059 & L1489 IRS & $6.63(6.18,7.09)$ & 50.9 & $(30.9,60.5)$ & $-3.00(-3.00,0.02)$ & 30.39 & $4.471(4.07,4.91)$ & -3.63 & 40.42 & 0.94 & 99 \\
\hline $20-001$ & $\mathrm{LkCa} 1$ & $0.07(0.04,0.15)$ & 4.9 & $(3.7,5.7)$ & $-3.00(-3.00,-1.94)$ & 2.66 & $0.232(0.20,0.37)$ & -3.80 & 4.35 & 0.57 & 18 \\
\hline $20-005$ & Anon 1 & $0.33(0.30,0.35)$ & 7.6 & $(6.4,9.1)$ & $-1.01(-1.26,-0.88)$ & 40.27 & $4.139(3.84,4.42)$ & -3.38 & 10.72 & 0.92 & 228 \\
\hline $20-000$ & IRAS $04108+2803 \mathrm{~A}$ & $\ldots$ & $\ldots$ & & $\ldots$ & & $\ldots$ & & & & $\ldots$ \\
\hline $20-022$ & IRAS $04108+2803 \mathrm{~B}$ & $5.68(2.99,8.19)$ & 4.5 & $(2.0,31.6)$ & -1.00 & 4.39 & $0.417(0.07,0.57)$ & -3.57 & 6.93 & 0.68 & 8 \\
\hline $20-000$ & $2 \mathrm{M} \mathrm{J} 04141188+28$ & 0.18 & 7.9 & & -3.00 & $\ldots$ & $<0.028$ & $<-3.31$ & 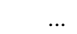 & & $\ldots$ \\
\hline 20-042 & V773 Tau ABC & $0.17(0.17,0.17)$ & 8.7 & $(8.4,9.1)$ & $-0.87(-0.93,-0.82)$ & 89.77 & $9.488(9.39,9.54)$ & -3.36 & 12.96 & 1.06 & 526 \\
\hline $20-043$ & FM Tau & $0.15(0.13,0.17)$ & 6.1 & $(3.9,9.6)$ & $-0.02(-0.29,0.11)$ & 4.59 & $0.532(0.51,0.56)$ & -3.52 & 18.47 & 1.12 & 78 \\
\hline $20-046$ & CW Tau & $6.49(4.02,8.12)$ & 4.5 & $(2.0,12.4)$ & $-3.00(-3.00,-2.21)$ & 33.14 & $2.844(0.28,4.00)$ & -3.17 & 4.07 & 1.76 & 2 \\
\hline $20-047$ & CIDA 1 & $0.22(0.03,0.47)$ & 5.0 & $(2.5,10.7)$ & -1.00 & 0.26 & $0.025(0.01,0.07)$ & & 7.57 & 0.68 & 3 \\
\hline $20-056$ & MHO 2/1 & $0.92(0.83,1.15)$ & 17.7 & $(10.4,28.9)$ & $-1.20(-3.00,-0.35)$ & 14.44 & $1.636(1.48,2.39)$ & -3.12 & 20.53 & 0.98 & 85 \\
\hline $20-058$ & MHO 3 & $0.61(0.48,1.02)$ & 10.0 & $(2.0,15.4)$ & $-1.40(-3.00,-0.74)$ & 4.46 & $0.459(0.36,2.18)$ & -3.88 & 11.76 & 0.86 & 20 \\
\hline $20-069$ & FO Tau AB & $0.25(0.17,0.77)$ & 12.5 & $(2.0,18.7)$ & $-2.01(-3.00,-0.37)$ & 0.64 & $0.065(0.05,0.52)$ & -4.66 & 12.33 & 1.29 & 4 \\
\hline $20-073$ & CIDA 2 & $0.13(0.06,0.27)$ & 6.0 & $(3.3,7.8)$ & $-2.87(-3.00,-1.49)$ & 1.96 & $0.178(0.14,0.36)$ & -3.84 & 5.34 & 0.80 & 18 \\
\hline $23-002$ & CY Tau & $0.00(0.00,0.17)$ & 6.7 & $(2.2,10.3)$ & $-1.05(-3.00,-0.36)$ & 1.32 & $0.133(0.13,0.29)$ & -4.16 & 9.42 & 1.71 & 10 \\
\hline 24-002 & CY Tau & 0.02 & 9.5 & $(6.6,13.7)$ & $-0.79(-2.00,-0.29)$ & 1.79 & $0.194(0.19,0.30)$ & -4.00 & 14.46 & 0.80 & 12 \\
\hline 23-004 & LkCa 5 & $0.02(0.01,0.03)$ & 7.9 & $(6.4,9.1)$ & $-1.33(-1.86,-0.91)$ & 4.32 & $0.432(0.41,0.45)$ & -3.52 & 9.67 & 0.59 & 85 \\
\hline 24-004 & LkCa 5 & $0.04(0.02,0.06)$ & 5.6 & $(3.7,8.3)$ & $-0.50(-0.96,-0.28)$ & 6.46 & $0.681(0.64,0.73)$ & -3.32 & 11.62 & 0.99 & 72 \\
\hline $23-008$ & CIDA 3 & $1.38(0.78,2.09)$ & 5.7 & $(2.0,31.6)$ & $-0.67(-1.67,0.45)$ & 2.67 & $0.277(0.12,1.62)$ & -3.29 & 10.45 & 1.15 & 11 \\
\hline $24-008$ & CIDA 3 & $0.85(0.67,1.09)$ & 31.6 & $(22.3,31.6)$ & -1.00 & 1.95 & $0.254(0.22,0.31)$ & -3.33 & 33.60 & 0.87 & 7 \\
\hline $23-015$ & V410 X3 & $0.13(0.07,0.25)$ & 4.9 & $(3.1,8.7)$ & $-1.22(-2.75,-0.71)$ & 0.63 & $0.059(0.05,0.10)$ & -3.74 & 6.67 & 0.76 & 13 \\
\hline $24-015$ & V410 X3 & $0.24(0.00,0.52)$ & 3.5 & $(2.0,9.9)$ & $-1.98(-3.00,-1.04)$ & 1.10 & $0.092(0.03,0.47)$ & -3.55 & 3.81 & 0.79 & 5 \\
\hline 23-018 & V410 A13 & $0.42(0.00,0.78)$ & 4.0 & $(2.0,20.6)$ & -1.00 & 0.10 & $0.010(0.00,0.03)$ & -4.18 & 6.21 & 1.11 & 2 \\
\hline $24-000$ & V410 A13 & 0.50 & 10.0 & & -1.00 & $\ldots$ & $<0.011$ & $<-4.14$ & $\ldots$ & $\ldots$ & $\ldots$ \\
\hline $23-000$ & V410 A24 & 4.37 & 10.0 & & -1.00 & $\ldots$ & $<0.042$ & $<-5.41$ & $\ldots$ & $\ldots$ & $\ldots$ \\
\hline $24-000$ & V410 A24 & 4.37 & 10.0 & & -1.00 & $\ldots$ & $<0.078$ & $<-5.14$ & & & \\
\hline 23-029 & V410 A 25 & $0.59(0.53,0.65)$ & 25.9 & $(23.1,29.1)$ & $-3.00(-3.00,-2.17)$ & 5.98 & $0.676(0.63,0.72)$ & -3.96 & 21.75 & 1.27 & 90 \\
\hline $24-027$ & V410 A25 & $0.57(0.49,0.66)$ & 33.6 & $(26.4,40.0)$ & $-3.00(-3.00,-1.10)$ & 4.79 & $0.587(0.55,0.64)$ & -4.02 & 27.79 & 1.11 & 47 \\
\hline 23-032 & V410 Tau ABC & $0.01(0.01,0.02)$ & 9.0 & $(7.7,10.1)$ & $-1.14(-1.43,-0.85)$ & 36.33 & $3.762(3.68,3.84)$ & -3.35 & 11.74 & 0.95 & 115 \\
\hline $24-028$ & V410 Tau ABC & $0.02(0.02,0.03)$ & 10.4 & $(9.4,10.8)$ & $-1.17(-1.28,-0.96)$ & 44.47 & $4.663(4.58,4.76)$ & -3.26 & 13.20 & 1.10 & 414 \\
\hline $23-033$ & DD Tau AB & $0.23(0.16,0.37)$ & 3.8 & $(2.0,31.6)$ & $0.93(0.19,1.00)$ & 0.69 & $0.093(0.08,0.11)$ & -4.15 & 37.84 & 1.37 & 6 \\
\hline 24-029 & DD Tau AB & $0.29(0.21,0.45)$ & 31.6 & $(2.0,31.6)$ & $0.13(-3.00,1.00)$ & 1.00 & $0.139(0.12,0.16)$ & -3.97 & 41.71 & 1.37 & 5 \\
\hline $23-035$ & CZ Tau AB & $0.34(0.24,0.43)$ & 5.0 & $(3.9,6.5)$ & $-3.00(-3.00,-2.57)$ & 4.86 & $0.423(0.28,0.64)$ & -3.39 & 4.41 & 1.09 & 28 \\
\hline 24-030 & CZ Tau AB & $0.33(0.24,0.37)$ & 5.0 & $(4.6,6.4)$ & $-3.00(-3.00,-2.73)$ & 6.22 & $0.547(0.37,0.64)$ & -3.28 & 4.45 & 1.86 & 22 \\
\hline $23-036$ & IRAS $04154+2823$ & $6.80(4.36,13.1)$ & 31.6 & $(2.0,31.6)$ & -1.00 & 1.44 & $0.188(0.13,0.88)$ & -3.43 & 33.60 & 0.89 & 8 \\
\hline $24-031$ & IRAS $04154+2823$ & $5.43(1.89,10.6)$ & 9.1 & $(2.0,31.6)$ & -1.00 & 1.56 & $0.164(0.03,0.72)$ & -3.48 & 12.58 & 1.16 & 1 \\
\hline $23-037$ & V410 X2 & $1.47(1.32,1.59)$ & 2.9 & $(2.0,5.2)$ & $-1.65(-1.82,-1.48)$ & 76.83 & $6.192(3.06,11.49)$ & -3.27 & 3.48 & 0.81 & 84 \\
\hline $24-032$ & $\mathrm{~V} 410 \mathrm{X} 2$ & $1.46(0.95,1.60)$ & 2.0 & $(2.0,14.1)$ & $-1.47(-1.75,-1.15)$ & 109.71 & $7.853(1.18,11.25)$ & -3.17 & 2.79 & 0.58 & 24 \\
\hline $23-045$ & V410 X4 & $0.94(0.79,1.16)$ & 15.0 & $(9.9,19.1)$ & $-3.00(-3.00,-1.34)$ & 2.10 & $0.211(0.17,0.30)$ & -4.38 & 12.73 & 0.74 & 19 \\
\hline $24-038$ & V410 X4 & $0.74(0.32,1.43)$ & 17.6 & $(2.0,31.6)$ & $-2.84(-3.00,0.42)$ & 2.06 & $0.213(0.13,1.69)$ & -4.37 & 15.15 & 0.12 & 4 \\
\hline 23-047 & V892 Tau & $0.94(0.89,0.98)$ & 12.0 & $(10.5,14.5)$ & $-1.18(-1.50,-1.04)$ & 86.14 & $9.207(8.51,9.90)$ & -4.51 & 14.86 & 1.17 & 377 \\
\hline $24-040$ & V892 Tau & $1.04(0.90,1.31)$ & 9.0 & $(3.3,13.7)$ & $-1.39(-1.97,-1.04)$ & 78.05 & $7.944(6.02,18.12)$ & -4.57 & 10.74 & 0.90 & 86 \\
\hline $23-048$ & LR 1 & $2.77(2.11,3.66)$ & 2.0 & $(2.0,31.6)$ & -1.00 & 5.33 & $0.417(0.29,0.55)$ & -3.64 & 3.63 & 0.59 & 5 \\
\hline $24-000$ & LR 1 & 4.16 & 10.0 & & -1.00 & & $<0.169$ & $<-4.03$ & $\ldots$ & $\ldots$ & $\ldots$ \\
\hline $23-050$ & V410 X7 & $0.80(0.70,0.92)$ & 12.6 & $(8.5,17.9)$ & $-1.34(-2.48,-0.88)$ & 8.60 & $0.913(0.78,1.16)$ & -3.32 & 14.68 & 0.98 & 44 \\
\hline $24-042$ & V410 X7 & $0.72(0.67,0.78)$ & 29.4 & $(19.4,32.5)$ & $-2.99(-3.00,-1.06)$ & 25.28 & $2.975(2.82,3.14)$ & -2.81 & 24.57 & 0.93 & 82 \\
\hline $23-000$ & V410 A20 & 4.27 & 10.0 & & -1.00 & $\ldots$ & $<0.075$ & $<-4.42$ & $\ldots$ & $\ldots$ & $\ldots$ \\
\hline $24-000$ & V410 A20 & 4.27 & 10.0 & & -1.00 & . & $<0.094$ & $<-4.32$ & $\ldots$ & $\ldots$ & $\ldots$ \\
\hline $23-056$ & Hubble 4 & $0.25(0.24,0.25)$ & 9.1 & $(8.6,9.6)$ & $-1.08(-1.17,-0.99)$ & 51.25 & $5.342(5.24,5.38)$ & -3.29 & 12.14 & 1.02 & 383 \\
\hline $24-047$ & Hubble 4 & $0.24(0.23,0.25)$ & 9.2 & $(8.6,10.0)$ & $-1.16(-1.29,-1.03)$ & 44.83 & $4.668(4.52,4.76)$ & -3.35 & 11.96 & 0.98 & 280 \\
\hline $23-000$ & KPNO-Tau 2 & 0.07 & 7.9 & & -3.00 & $\ldots$ & $<0.012$ & $<-3.38$ & $\ldots$ & $\ldots$ & $\ldots$ \\
\hline $24-000$ & KPNO-Tau 2 & 0.07 & 7.9 & & -3.00 & $\ldots$ & $<0.012$ & $<-3.38$ & $\ldots$ & $\ldots$ & $\ldots$ \\
\hline $23-000$ & CoKu Tau 1 & $\ldots$ & $\ldots$ & & $\ldots$ & $\ldots$ & $\ldots$ & $\ldots$ & $\ldots$ & $\ldots$ & ... \\
\hline $24-000$ & CoKu Tau 1 & $\ldots$ & $\ldots$ & & $\ldots$ & $\ldots$ & $\ldots$ & $\ldots$ & $\ldots$ & $\ldots$ & $\ldots$ \\
\hline 23-061 & V410 X6 & $0.36(0.26,0.43)$ & 3.2 & $(2.1,4.6)$ & -1.00 & 1.39 & $0.124(0.08,0.17)$ & -3.79 & 5.15 & 1.26 & 13 \\
\hline
\end{tabular}


M. Güdel et al.: XMM-Newton extended survey of Taurus, Online Material p 9

Table 5. continued.

\begin{tabular}{|c|c|c|c|c|c|c|c|c|c|c|c|}
\hline XEST & Name & $\begin{array}{l}N_{\mathrm{H}}(1 \sigma \text { range }) \\
\left(10^{22} \mathrm{~cm}^{-2}\right)\end{array}$ & $\begin{array}{l}T_{0} \quad(1 \sigma \text { range }) \\
(\mathrm{MK})\end{array}$ & (1 $\sigma$ range $)$ & $\begin{array}{r}\mathrm{EM}_{t}^{a} \\
\left(10^{52}\right) \\
\end{array}$ & $\begin{array}{l}L_{\mathrm{X}}^{b} \\
\left(10^{30} \mathrm{e}\right.\end{array}$ & $\begin{array}{l}\text { (range) } \\
\left.\mathrm{rg} \mathrm{s}^{-1}\right)\end{array}$ & $\begin{array}{r}\log \\
L_{\mathrm{X}} / L_{*}\end{array}$ & $\begin{array}{r}T_{\mathrm{av}} \\
(\mathrm{MK}) \\
\end{array}$ & $\chi_{\text {red }}^{2}$ & d.o.f. \\
\hline $24-054$ & V410 X6 & $0.45(0.32,0.63)$ & $5.0 \quad(2.4,9.1)$ & $-1.19(-2.55,-0.71)$ & 2.93 & 0.278 & $(0.18,0.57)$ & -3.44 & 6.86 & 1.21 & 9 \\
\hline $23-063$ & V410 X5 & $0.45(0.37,0.56)$ & $(4.6,11.6)$ & $-1.16(-1.90,-0.77)$ & 3.87 & 0.387 & $(0.31,0.52)$ & -2.92 & 9.30 & 1.17 & 32 \\
\hline $24-055$ & V410 X5 & $0.61(0.52,0.72)$ & $(2.2,7.0)$ & $-0.50(-0.76,-0.30)$ & 11.07 & 1.115 & $(0.87,1.59)$ & -2.46 & 9.16 & 0.94 & 41 \\
\hline $23-067$ & FQ Tau AB & $0.50(0.22,0.93)$ & $(2.0,10.1)$ & $-3.00(-3.00,-1.51)$ & 1.36 & 0.120 & $(0.05,0.83)$ & -3.83 & 4.46 & 1.79 & 6 \\
\hline $24-058$ & FQ Tau AB & 0.35 & $(2.0,10.0)$ & -3.00 & 5.59 & 0.049 & $(0.04,0.16)$ & -4.22 & 5.17 & 0.87 & 1 \\
\hline $28-100$ & BP Tau & $0.06(0.06,0.07)$ & $(6.7,7.6)$ & $-0.67(-0.73,-0.60)$ & 12.84 & 1.365 & $(1.35,1.40)$ & -3.43 & 12.34 & 1.10 & 430 \\
\hline $23-074$ & V819 Tau AB & $0.21(0.20,0.23)$ & $(4.3,5.4)$ & $-0.99(-1.10,-0.90)$ & 25.41 & 2.445 & $(2.33,2.61)$ & -3.16 & 7.45 & 1.04 & 199 \\
\hline $24-061$ & V819 Tau AB & $0.22(0.18,0.26)$ & $(3.5,5.7)$ & $-1.00(-1.13,-0.87)$ & 23.24 & 2.205 & $(1.91,2.66)$ & -3.20 & 6.87 & 0.90 & 110 \\
\hline $16-000$ & IRAS $04166+2706$ & $\ldots$ & $\ldots$ & $\ldots$ & $\ldots$ & $\ldots$ & & $\ldots$ & $\ldots$ & $\ldots$ & $\ldots$ \\
\hline $16-000$ & IRAS $04169+2702$ & $\ldots$ & $\ldots$ & $\ldots$ & $\ldots$ & $\ldots$ & & $\ldots$ & $\ldots$ & $\ldots$ & $\ldots$ \\
\hline $11-000$ & CFHT-Tau 19 & 1.31 & 7.9 & -3.00 & $\ldots$ & $<0.047$ & & $<-3.77$ & $\ldots$ & $\ldots$ & $\ldots$ \\
\hline $11-000$ & IRAS $04181+2655$ & $\ldots$ & $\ldots$ & $\ldots$ & $\ldots$ & $\ldots$ & & $\ldots$ & $\ldots$ & $\ldots$ & $\ldots$ \\
\hline $11-000$ & IRAS $04181+2654 \mathrm{AB}$ & $\ldots$ & $\ldots$ & $\ldots$ & $\ldots$ & $\ldots$ & & $\ldots$ & $\ldots$ & $\ldots$ & $\ldots$ \\
\hline $11-023$ & 2M J04213459 & 0.31 & $(3.8,10.0)$ & $-1.37(-3.00,-0.91)$ & 0.49 & 0.043 & $(0.04,0.05)$ & -3.76 & 6.79 & 0.90 & 4 \\
\hline $01-028$ & IRAS $04187+1927$ & $0.62(0.55,0.70)$ & $(4.7,12.1)$ & $-1.02(-1.28,-0.84)$ & 9.09 & 0.909 & $(0.75,1.15)$ & $\ldots$ & 9.15 & 0.94 & 79 \\
\hline $11-037$ & CFHT-Tau 10 & 0.65 & 10.0 & -3.00 & 0.16 & 0.015 & & -3.73 & 8.59 & $\ldots$ & $\ldots$ \\
\hline $11-000$ & 2M J04215450+2652 & 0.54 & 7.9 & -3.00 & $\ldots$ & $<0.008$ & & $<-3.17$ & $\ldots$ & $\ldots$ & $\ldots$ \\
\hline $21-038$ & RY Tau & $0.77(0.70,0.86)$ & $(2.0,4.2)$ & $0.20 \quad(-0.03,0.41)$ & 49.07 & 5.520 & $(4.82,6.38)$ & -3.72 & 15.70 & 1.18 & 158 \\
\hline $21-039$ & HD 283572 & $0.08(0.07,0.08)$ & $10.4(10.0,10.7)$ & $-0.94(-1.02,-0.87)$ & 114.33 & 13.003 & $(2.68,13.05)$ & -3.28 & 14.43 & 1.12 & 522 \\
\hline $01-045$ & T Tau N(+Sab) & $0.27(0.27,0.28)$ & $11.3(10.8,11.8)$ & $-0.65(-0.72,-0.58)$ & 71.84 & 8.048 & $(7.99,8.15)$ & -3.63 & 17.62 & 1.21 & 627 \\
\hline $11-054$ & Haro 6-5 B & $19.3(12.9,23.8)$ & $6.6 \quad(2.0,16.7)$ & $-3.00(-3.00,-2.30)$ & 18.95 & 17.551 & $(1.49,22.38)$ & -1.01 & 5.74 & 1.26 & 7 \\
\hline $11-057$ & FS Tau AC & $1.42(1.34,1.48)$ & $39.8(34.6,44.8)$ & $-2.90(-3.00,-1.53)$ & 24.99 & 3.224 & $(3.09,3.36)$ & -2.58 & 32.76 & 1.13 & 155 \\
\hline 21-044 & LkCa 21 & $0.08(0.06,0.10)$ & $8.5 \quad(6.0,9.7)$ & $-2.21(-3.00,-1.31)$ & 6.65 & 0.646 & $(0.60,0.71)$ & -3.57 & 8.18 & 1.07 & 82 \\
\hline 01-054 & RX J0422.1+1934 & $0.28(0.27,0.28)$ & $13.6(12.8,14.5)$ & $-1.64(-1.93,-1.22)$ & 29.59 & 3.110 & $(3.05,3.12)$ & & 14.46 & 1.05 & 411 \\
\hline $01-062$ & 2M J04221332+1934 & $0.37(0.19,0.60)$ & $4.4 \quad(2.5,7.7)$ & $-3.00(-3.00,-1.63)$ & 0.67 & 0.057 & $(0.03,0.22)$ & -3.07 & 3.92 & 1.06 & 6 \\
\hline $11-079$ & CFHT-Tau 21 & 1.19 & $31.6(23.4,31.6)$ & -1.00 & 1.16 & 0.151 & $(0.15,0.15)$ & -3.99 & 33.60 & 0.73 & 8 \\
\hline $02-013$ & FV Tau AB & $1.20(0.87,1.50)$ & $2.0 \quad(2.0,31.6)$ & $0.32(-0.41,1.00)$ & 4.51 & 0.532 & $(0.37,0.72)$ & -3.94 & 18.76 & 1.06 & 13 \\
\hline $02-000$ & FV Tau/c AB & 0.59 & 10.0 & -1.00 & & $<0.047$ & & $<-4.24$ & & & $\ldots$ \\
\hline $02-016$ & KPNO-Tau 13 & $0.32(0.23,0.45)$ & $5.0 \quad(2.2,8.1)$ & $-0.97(-1.93,-0.53)$ & 1.42 & 0.138 & $(0.10,0.24)$ & -3.62 & 7.71 & 0.79 & 11 \\
\hline $02-000$ & DG Tau B & $\ldots$ & $\ldots$ & $\ldots$ & $\ldots$ & $\ldots$ & & $\ldots$ & $\ldots$ & $\ldots$ & $\ldots$ \\
\hline $02-022$ & DG Tau A & $\ldots$ & $\ldots$ & $\ldots$ & $\ldots$ & $\ldots$ & & $\ldots$ & $\ldots$ & $\ldots$ & $\ldots$ \\
\hline $02-000$ & KPNO-Tau 4 & 0.44 & 7.9 & -3.00 & $\ldots$ & $<0.013$ & & $<-3.09$ & $\ldots$ & $\ldots$ & $\ldots$ \\
\hline $02-000$ & IRAS $04248+2612 \mathrm{AB}$ & $\ldots$ & $\ldots$ & $\ldots$ & $\ldots$ & $\ldots$ & & $\ldots$ & $\ldots$ & $\ldots$ & $\ldots$ \\
\hline $15-020$ & JH 507 & $0.26(0.19,0.36)$ & $(2.7,5.2)$ & $-1.56(-1.97,-1.25)$ & 5.20 & 0.455 & $(0.34,0.75)$ & -3.55 & 4.62 & 0.47 & 31 \\
\hline $13-004$ & GV Tau AB & $\ldots$ & $\ldots$ & $\ldots$ & $\ldots$ & $\ldots$ & & $\ldots$ & $\ldots$ & $\ldots$ & $\ldots$ \\
\hline $13-000$ & IRAS $04264+2433$ & $\ldots$ & $\ldots$ & $\ldots$ & $\ldots$ & $\ldots$ & & $\ldots$ & $\ldots$ & $\ldots$ & $\ldots$ \\
\hline $15-040$ & DH Tau AB & $0.20(0.19,0.21)$ & $11.5(11.0,12.1)$ & $-1.38(-1.49,-1.26)$ & 80.60 & 8.458 & $(8.23,8.64)$ & -2.41 & 13.42 & 1.06 & 381 \\
\hline $15-042$ & DI Tau AB & $0.14(0.13,0.16)$ & $9.5 \quad(8.4,11.3)$ & $-1.43(-2.01,-1.18)$ & 15.39 & 1.568 & $(1.51,1.65)$ & -3.39 & 11.07 & 0.92 & 111 \\
\hline $15-044$ & KPNO-Tau 5 & 0.01 & $(4.6,12.6)$ & $-3.00(-3.00,-0.66)$ & 0.10 & 0.010 & $(0.01,0.01)$ & -3.95 & 6.88 & 1.11 & 2 \\
\hline $14-006$ & IQ Tau A & $0.49(0.36,0.80)$ & $10.1(2.0,19.6)$ & $-0.95(-3.00,-0.06)$ & 3.89 & 0.416 & $(0.33,1.17)$ & -3.91 & 14.06 & 1.57 & 9 \\
\hline $13-000$ & CFHT-Tau 20 & 0.65 & 7.9 & -3.00 & $\ldots$ & $<0.017$ & & $<-4.49$ & $\ldots$ & $\ldots$ & $\ldots$ \\
\hline $14-000$ & KPNO-Tau 6 & 0.16 & 7.9 & -3.00 & $\ldots$ & $<0.008$ & & $<-3.17$ & $\ldots$ & & $\ldots$ \\
\hline $13-035$ & FX Tau AB & $0.26(0.18,0.55)$ & $(2.0,10.1)$ & $-3.00(-3.00,-0.94)$ & 5.26 & 0.502 & $(0.39,2.36)$ & -3.89 & 6.86 & 0.98 & 5 \\
\hline $14-057$ & DK Tau AB & $0.25(0.23,0.27)$ & $(6.7,13.3)$ & $-0.81(-1.57,-0.52)$ & 8.54 & 0.916 & $(0.87,0.96)$ & -3.74 & 13.90 & 0.86 & 106 \\
\hline $14-000$ & KPNO-Tau 7 & 0.01 & 7.9 & -3.00 & $\ldots$ & $<0.006$ & & $<-3.33$ & $\ldots$ & $\ldots$ & $\ldots$ \\
\hline $22-013$ & MHO 9 & $0.05(0.02,0.08)$ & $(5.6,7.9)$ & $-3.00(-3.00,-1.92)$ & 0.86 & 0.080 & $(0.07,0.09)$ & -4.02 & 5.93 & 1.05 & 20 \\
\hline $22-021$ & MHO 4 & $0.16(0.11,0.22)$ & $6.1 \quad(3.8,11.2)$ & $-1.15(-3.00,-0.60)$ & 1.25 & 0.123 & $(0.10,0.16)$ & -3.18 & 8.26 & 0.73 & 16 \\
\hline $22-040$ & L1551 IRS5 & $0.17(0.11,0.29)$ & $31.6(21.4,31.6)$ & -1.00 & 0.17 & 0.021 & $(0.02,0.02)$ & -5.68 & 33.60 & 1.40 & 6 \\
\hline $22-042$ & LkHa 358 & $3.01(2.26,4.05)$ & $3.0 \quad(2.0,30.9)$ & -1.00 & 4.58 & 0.404 & $(0.29,0.53)$ & -3.75 & 4.99 & 0.40 & 11 \\
\hline $22-000$ & HН 30 & & $\ldots$ & & & & & & & & \\
\hline $22-043$ & HL Tau & $2.81(2.59,3.04)$ & $20.1(13.6,29.9)$ & $-1.40(-3.00,-0.80)$ & 26.84 & 3.838 & $(3.22,4.73)$ & -3.19 & 21.80 & 1.14 & 87 \\
\hline $22-047$ & XZ Tau AB & $0.28(0.23,0.33)$ & $8.5 \quad(6.0,11.5)$ & $-0.90(-1.45,-0.62)$ & 9.14 & 0.962 & $(0.86,1.12)$ & -3.12 & 12.49 & 1.07 & 38 \\
\hline $22-056$ & L1551 NE & $7.66(3.96,13.8)$ & 31.6 & -1.00 & 0.46 & 0.059 & $(0.03,0.09)$ & $\ldots$ & 33.60 & 0.10 & 1 \\
\hline 03-005 & HK Tau AB & $0.92(0.49,1.89)$ & $63.1(14.1,63.1)$ & -1.00 & 0.53 & 0.079 & $(0.06,0.12)$ & -4.44 & 51.83 & 3.19 & 1 \\
\hline $22-070$ & V710 Tau BA & $0.28(0.26,0.30)$ & $7.9 \quad(6.7,8.8)$ & $-1.44(-1.71,-1.22)$ & 13.81 & 1.378 & $(1.32,1.49)$ & -3.49 & 9.33 & 0.94 & 158 \\
\hline $19-009$ & JH 665 & $0.14(0.05,0.37)$ & $(2.0,11.8)$ & $-0.94(-3.00,-0.26)$ & 0.81 & 0.079 & $(0.06,0.23)$ & -4.10 & 7.78 & 0.58 & 3 \\
\hline $22-089$ & L1551 51 & $0.11(0.10,0.12)$ & $(7.0,8.4)$ & $-1.63(-1.88,-1.42)$ & 18.67 & 1.841 & $(1.78,1.91)$ & -2.99 & 8.61 & 1.13 & 204 \\
\hline $22-097$ & V827 Tau & $0.06(0.05,0.06)$ & $(8.7,10.7)$ & $-0.74(-0.96,-0.65)$ & 37.02 & 4.010 & $(3.88,4.01)$ & -3.02 & 14.64 & 1.11 & 273 \\
\hline 03-016 & Haro 6-13 & $1.26(0.44,1.41)$ & $(2.0,31.6)$ & -1.00 & 9.49 & 0.799 & $(0.14,0.91)$ & -4.01 & 4.29 & 1.04 & 6 \\
\hline
\end{tabular}


Table 5. continued.

\begin{tabular}{|c|c|c|c|c|c|c|c|c|c|c|}
\hline XEST & Name & $\begin{array}{l}N_{\mathrm{H}}(1 \sigma \text { range }) \\
\left(10^{22} \mathrm{~cm}^{-2}\right)\end{array}$ & $\begin{array}{l}T_{0} \quad(1 \sigma \text { range }) \\
(\mathrm{MK})\end{array}$ & ( $1 \sigma$ range $)$ & $\begin{array}{r}\mathrm{EM}_{t}^{a} \\
\left(10^{52}\right) \\
\end{array}$ & $\begin{array}{l}L_{\mathrm{X}}^{b} \quad \text { (range) } \\
\left(10^{30} \mathrm{erg} \mathrm{s}^{-1}\right)\end{array}$ & $\begin{array}{r}\log \\
L_{\mathrm{X}} / L_{*}\end{array}$ & $\begin{array}{r}T_{\mathrm{av}} \\
(\mathrm{MK}) \\
\end{array}$ & $\chi_{\text {red }}^{2}$ & d.o.f. \\
\hline $22-100$ & V826 Tau & $0.07(0.06,0.07)$ & $9.1 \quad(8.6,9.4)$ & $-1.26(-1.36,-1.16)$ & 44.06 & $4.523(4.42,4.57)$ & -2.90 & 11.27 & 1.12 & 359 \\
\hline $22-101$ & MHO 5 & $0.09(0.06,0.14)$ & $(4.2,8.6)$ & $-1.62(-3.00,-1.09)$ & 1.18 & $0.111(0.10,0.14)$ & -3.58 & 6.63 & 0.95 & 31 \\
\hline 03-017 & CFHT-Tau 7 & 0.01 & $(5.9,12.0)$ & $-3.00(-3.00,-0.90)$ & 0.21 & $0.020(0.02,0.02)$ & -4.07 & 7.03 & 1.82 & 3 \\
\hline 03-019 & V928 Tau AB & $0.38(0.31,0.44)$ & $(3.9,7.0)$ & $-1.40(-1.78,-1.23)$ & 11.21 & $1.046(0.81,1.33)$ & -3.71 & 6.27 & 0.93 & 46 \\
\hline 03-022 & FY Tau & $0.31(0.28,0.36)$ & $13.7(8.7,19.6)$ & $-0.99(-2.31,-0.40)$ & 7.30 & $0.807(0.76,0.90)$ & -3.68 & 17.73 & 0.54 & 34 \\
\hline $03-023$ & FZ Tau & $0.53(0.33,0.82)$ & $4.8 \quad(2.0,12.0)$ & $-1.55(-2.44,-1.05)$ & 7.05 & $0.644(0.35,2.91)$ & -3.77 & 5.65 & 0.70 & 16 \\
\hline $17-002$ & IRAS $04295+2251$ & $3.75(2.74,5.26)$ & $16.8(5.2,27.0)$ & $-3.00(-3.00,-1.21)$ & 14.56 & $1.489(0.82,4.80)$ & -3.49 & 14.28 & 0.46 & 7 \\
\hline 19-049 & UZ Tau E+W(AB) & $0.44(0.29,0.53)$ & $2.7 \quad(2.0,7.4)$ & $-0.75(-0.95,-0.58)$ & 9.98 & $0.890(0.51,1.35)$ & -3.59 & 5.44 & 0.74 & 61 \\
\hline $17-009$ & JH 112 & $0.72(0.57,0.86)$ & $(2.0,7.8)$ & $-0.90(-1.20,-0.61)$ & 8.71 & $0.820(0.52,1.48)$ & -3.54 & 6.58 & 0.64 & 22 \\
\hline 03-031 & CFHT-Tau 5 & 0.84 & $8.1 \quad(3.5,11.5)$ & $-3.00(-3.00,-1.27)$ & 1.91 & $0.181(0.14,0.26)$ & -3.20 & 7.03 & 1.26 & 4 \\
\hline 04-003 & CFHT-Tau 5 & $0.84(0.51,1.48)$ & $11.9(2.0,17.8)$ & $-3.00(-3.00,-1.07)$ & 2.51 & $0.247(0.14,2.28)$ & -3.07 & 10.14 & 0.63 & 7 \\
\hline 03-035 & MHO 8 & $0.21(0.13,0.34)$ & $4.0 \quad(2.1,6.1)$ & -1.00 & 0.70 & $0.065(0.05,0.12)$ & -4.00 & 6.21 & 2.00 & 3 \\
\hline 04-009 & MHO 8 & $0.68(0.46,0.82)$ & $(2.0,4.4)$ & $-3.00(-3.00,-2.51)$ & 6.18 & $0.444(0.13,0.93)$ & -3.17 & 2.43 & 0.97 & 5 \\
\hline 04-010 & GH Tau AB & 0.09 & $12.6(9.2,18.8)$ & -1.00 & 1.00 & $0.109(0.10,0.12)$ & -4.46 & 16.59 & 0.71 & 7 \\
\hline 04-012 & V807 Tau SNab & $0.10(0.08,0.12)$ & $5.4 \quad(4.4,6.4)$ & $-1.18(-1.40,-0.99)$ & 10.92 & $1.049(0.97,1.15)$ & -3.89 & 7.35 & 1.06 & 84 \\
\hline $18-004$ & KPNO-Tau 14 & $0.85(0.69,1.31)$ & $12.6(2.0,21.3)$ & $-1.00(-2.30,-0.40)$ & 8.44 & $0.923(0.76,3.08)$ & -2.66 & 16.50 & 1.05 & 27 \\
\hline $17-000$ & CFHT-Tau 12 & 0.61 & 7.9 & -3.00 & & $<0.007$ & $<-4.30$ & & & \\
\hline 04-016 & V830 Tau & $0.05(0.04,0.05)$ & $(8.4,9.6)$ & $-0.53(-0.64,-0.42)$ & 46.62 & $5.181(5.07,5.23)$ & -2.76 & 16.23 & 1.22 & 309 \\
\hline $18-000$ & IRAS S04301+261 & 1.13 & 10.0 & -1.00 & $\ldots$ & $<0.023$ & $<-3.62$ & $\ldots$ & $\ldots$ & $\ldots$ \\
\hline $17-000$ & IRAS $04302+2247$ & $\ldots$ & $\ldots$ & $\ldots$ & $\ldots$ & $\ldots$ & $\ldots$ & $\ldots$ & $\ldots$ & $\ldots$ \\
\hline $17-027$ & IRAS $04303+2240$ & $1.66(1.44,1.79)$ & $(2.0,6.8)$ & $0.07 \quad(-0.10,0.48)$ & 41.37 & $5.006(3.50,5.97)$ & -3.23 & 12.95 & 0.95 & 114 \\
\hline 04-034 & GI Tau & $0.38(0.33,0.45)$ & $(2.0,6.8)$ & $-0.19(-0.33,-0.05)$ & 7.76 & $0.833(0.73,1.06)$ & -3.66 & 12.52 & 0.76 & 49 \\
\hline 04-035 & GK Tau AB & $0.45(0.41,0.71)$ & $(2.0,9.5)$ & $-1.03(-1.31,-0.72)$ & 14.24 & $1.471(1.33,4.41)$ & -3.56 & 10.92 & 1.02 & 80 \\
\hline 18-019 & IS Tau AB & $0.40(0.35,0.50)$ & $14.2(8.4,16.6)$ & $-2.49(-3.00,-1.17)$ & 6.48 & $0.658(0.60,0.85)$ & -3.59 & 12.83 & 1.07 & 56 \\
\hline $17-058$ & CI Tau & $0.55(0.38,1.18)$ & $17.7(2.0,31.6)$ & -1.00 & 1.69 & $0.195(0.16,0.89)$ & -4.23 & 21.78 & 0.68 & 9 \\
\hline $18-030$ & IT Tau AB & $0.69(0.66,0.72)$ & $22.6(17.1,34.5)$ & $-0.86(-3.00,-0.38)$ & 53.13 & $6.492(6.28,6.73)$ & -3.15 & 27.22 & 0.92 & 427 \\
\hline $17-066$ & JH 108 & $0.20(0.17,0.24)$ & $9.4 \quad(6.5,12.5)$ & $-0.85(-1.49,-0.44)$ & 11.48 & $1.233(1.14,1.36)$ & -2.97 & 13.93 & 1.07 & 38 \\
\hline $17-068$ & CFHT-BD Tau 1 & 0.56 & $17.0(12.6,25.7)$ & -1.00 & 1.48 & $0.169(0.17,0.17)$ & -2.59 & 21.04 & 1.10 & 4 \\
\hline $25-026$ & AA Tau & $1.09(0.96,1.20)$ & $25.7(20.6,31.0)$ & $-3.00(-3.00,-1.79)$ & 11.00 & $1.241(1.11,1.36)$ & -3.39 & 21.55 & 0.91 & 42 \\
\hline 09-010 & HO Tau AB & 0.20 & $5.3 \quad(3.7,8.0)$ & -1.00 & 0.48 & $0.047(0.05,0.05)$ & -4.14 & 8.01 & 1.06 & 5 \\
\hline 08-019 & FF Tau AB & $0.31(0.26,0.41)$ & $8.0 \quad(4.7,10.6)$ & $-1.50(-2.47,-1.00)$ & 7.99 & $0.796(0.69,1.12)$ & -3.52 & 9.21 & 1.07 & 59 \\
\hline $12-040$ & DN Tau & $0.07(0.07,0.08)$ & $10.5(9.5,11.5)$ & $-1.25(-1.51,-1.02)$ & 11.03 & $1.155(1.14,1.17)$ & -3.52 & 12.93 & 1.12 & 227 \\
\hline $12-000$ & IRAS $04325+2402 \mathrm{AB}$ & $\ldots$ & $\ldots$ & $\ldots$ & & $\ldots$ & $\ldots$ & $\ldots$ & & $\ldots$ \\
\hline $12-059$ & CoKu Tau 3 AB & $0.38(0.37,0.39)$ & $14.2(13.3,15.0)$ & $-2.07(-2.36,-1.80)$ & 56.63 & $5.851(5.71,5.94)$ & -2.81 & 13.80 & 1.06 & 388 \\
\hline 09-022 & KPNO-Tau 8 & $0.11(0.08,0.16)$ & $3.4 \quad(2.0,5.8)$ & $-0.11(-0.23,0.01)$ & 4.66 & $0.504(0.45,0.60)$ & -2.21 & 12.62 & 0.75 & 59 \\
\hline 08-037 & HQ Tau AB & $0.40(0.37,0.45)$ & $(6.4,9.6)$ & $-1.16(-1.43,-0.99)$ & 24.25 & $2.476(2.29,2.84)$ & $\ldots$ & 10.57 & 1.07 & 135 \\
\hline 09-026 & HQ Tau AB & $0.56(0.52,0.61)$ & $(4.7,7.5)$ & $-0.84(-0.95,-0.71)$ & 79.94 & $8.161(7.43,9.56)$ & $\ldots$ & 10.05 & 0.84 & 166 \\
\hline $08-043$ & KPNO-Tau 15 & $0.38(0.36,0.43)$ & $13.7(7.1,15.0)$ & $-0.33(-0.45,0.10)$ & 21.46 & $2.624(2.49,2.78)$ & -2.31 & 23.46 & 0.91 & 248 \\
\hline 09-031 & KPNO-Tau 15 & $0.37(0.26,0.52)$ & $6.3 \quad(3.6,10.1)$ & $-1.07(-2.05,-0.59)$ & 4.85 & $0.483(0.37,0.77)$ & -3.05 & 8.92 & 1.22 & 11 \\
\hline $08-000$ & KPNO-Tau 9 & 0.01 & 7.9 & -3.00 & $\ldots$ & $<0.004$ & $<-3.13$ & $\ldots$ & $\ldots$ & $\ldots$ \\
\hline 09-000 & KPNO-Tau 9 & 0.01 & 7.9 & -3.00 & $\ldots$ & $<0.007$ & $<-2.89$ & $\ldots$ & $\ldots$ & $\ldots$ \\
\hline 08-048 & HP Tau AB & $0.53(0.48,0.60)$ & $(6.6,11.4)$ & $-1.09(-1.44,-0.89)$ & 24.55 & $2.550(2.28,3.03)$ & -3.33 & 11.81 & 1.07 & 129 \\
\hline 08-051a & HP Tau/G3 AB & $0.41(0.39,0.42)$ & $(8.6,9.9)$ & $-1.37(-1.46,-1.28)$ & 12.85 & $1.293(1.09,1.39)$ & -3.33 & 11.03 & 1.04 & 382 \\
\hline 08-051 & HP Tau/G2 & $0.41(0.39,0.42)$ & $(8.6,9.9)$ & $-1.37(-1.46,-1.28)$ & 94.61 & $9.653(9.15,9.90)$ & -3.41 & 11.03 & 1.04 & 382 \\
\hline $08-058$ & Haro 6-28 AB & $0.38(0.33,0.47)$ & $10.0(6.5,12.2)$ & $-2.10(-3.00,-1.16)$ & 2.49 & $0.248(0.22,0.34)$ & -3.27 & 9.80 & 0.85 & 32 \\
\hline 08-000 & CFHT-BD Tau 2 & 0.01 & 7.9 & -3.00 & $\ldots$ & $<0.004$ & $<-4.02$ & $\ldots$ & $\ldots$ & $\ldots$ \\
\hline $08-080$ & CFHT-BD Tau 3 & 0.18 & $(4.0,17.8)$ & -3.00 & 0.13 & $0.012(0.01,0.01)$ & -3.36 & 6.88 & 0.16 & 1 \\
\hline $05-005$ & CFHT-Tau 6 & $0.47(0.33,0.64)$ & 7.9 & -3.00 & 0.75 & $0.071(0.04,0.09)$ & -3.11 & 6.88 & 1.53 & 3 \\
\hline $05-000$ & IRAS $04361+2547$ & $\ldots$ & $\ldots$ & $\ldots$ & $\ldots$ & $\ldots$ & $\ldots$ & $\ldots$ & $\ldots$ & $\ldots$ \\
\hline 05-013 & GN Tau AB & $2.34(1.48,2.80)$ & $(2.0,17.7)$ & -1.00 & 8.35 & $0.785(0.21,1.00)$ & -3.55 & 6.52 & 1.33 & 6 \\
\hline 05-017 & IRAS $04365+2535$ & $30.7(20.0,51.8)$ & 10.0 & -1.00 & 18.70 & $1.986(1.23,3.52)$ & -3.63 & 13.65 & 0.28 & 2 \\
\hline 05-024 & IRAS $04369+2539$ & $7.69(5.40,8.71)$ & $6.4 \quad(2.0,31.6)$ & -1.00 & 46.50 & $4.671(1.18,5.55)$ & -4.23 & 9.32 & 1.12 & 7 \\
\hline 07-011 & JH 223 & 0.09 & $9.3 \quad(7.6,10.6)$ & $-3.00(-3.00,-1.74)$ & 0.66 & $0.064(0.06,0.07)$ & -4.03 & 8.00 & 1.22 & 10 \\
\hline $07-022$ & Haro 6-32 & $0.08(0.03,0.14)$ & $12.0(5.8,14.7)$ & $-3.00(-3.00,-0.76)$ & 1.02 & $0.101(0.09,0.12)$ & -3.66 & 10.28 & 0.50 & 5 \\
\hline 07-000 & ITG 33 A & $\ldots$ & $\ldots$ & $\ldots$ & $\ldots$ & $\ldots$ & & $\ldots$ & $\ldots$ & $\ldots$ \\
\hline 07-000 & CFHT-Tau 8 & 0.32 & 7.9 & -3.00 & $\ldots$ & $<0.008$ & $<-4.06$ & $\ldots$ & $\ldots$ & $\ldots$ \\
\hline 07-000 & IRAS $04381+2540$ & $\ldots$ & $\ldots$ & $\ldots$ & $\ldots$ & $\ldots$ & $\ldots$ & $\ldots$ & $\ldots$ & $\ldots$ \\
\hline 07-041 & IRAS $04385+2550 \mathrm{AB}$ & $0.80(0.69,1.02)$ & $31.6(17.9,31.6)$ & -1.00 & 3.08 & $0.401(0.37,0.50)$ & -3.24 & 33.60 & 0.74 & 10 \\
\hline $10-017$ & CoKuLk332/G2 AB & $0.53(0.49,0.59)$ & $6.3 \quad(4.8,7.3)$ & $-1.14(-1.26,-1.02)$ & 32.92 & $3.257(2.91,3.94)$ & -3.11 & 8.59 & 0.83 & 163 \\
\hline
\end{tabular}


Table 5. continued.

\begin{tabular}{|c|c|c|c|c|c|c|c|c|c|c|}
\hline XEST & Name & $\begin{array}{l}N_{\mathrm{H}}(1 \sigma \text { range }) \\
\left(10^{22} \mathrm{~cm}^{-2}\right)\end{array}$ & $\begin{array}{l}T_{0} \quad(1 \sigma \text { range }) \\
(\mathrm{MK})\end{array}$ & ( $1 \sigma$ range $)$ & $\begin{array}{c}\mathrm{EM}_{t}^{a} \\
\left(10^{52}\right)\end{array}$ & $\begin{array}{l}L_{\mathrm{X}}^{b} \quad \text { (range) } \\
\left(10^{30} \mathrm{erg} \mathrm{s}^{-1}\right)\end{array}$ & $\begin{array}{r}\log \\
L_{\mathrm{X}} / L_{*}\end{array}$ & $\begin{array}{r}T_{\mathrm{av}} \\
(\mathrm{MK})\end{array}$ & $\chi_{\mathrm{red}}^{2}$ & d.o.f. \\
\hline $10-018$ & CoKuLk332/G1 AB & $0.33(0.24,0.58)$ & $10.0(2.0,15.5)$ & $-0.70(-1.73,-0.26)$ & 4.50 & $0.493(0.42,1.11)$ & $\frac{-127}{-4.12}$ & $\frac{15.78}{15.78}$ & 0.64 & 21 \\
\hline $10-020$ & V955 Tau AB & $0.89(0.70,1.30)$ & $9.5 \quad(2.0,21.1)$ & $-0.83(-1.90,-0.41)$ & 15.07 & $1.622(1.23,4.55)$ & -3.38 & 14.25 & 0.91 & 36 \\
\hline $10-034$ & CIDA 7 & 0.32 & $(2.4,7.7)$ & $-1.88(-3.00,-0.90)$ & 0.46 & $0.040(0.04,0.05)$ & -3.68 & 4.70 & 0.76 & 6 \\
\hline $10-045$ & DP Tau & & ... & $\ldots$ & & $\ldots$ & & & & $\ldots$ \\
\hline $10-060$ & GO Tau & $0.31(0.25,0.44)$ & $6.2 \quad(2.0,10.2)$ & $-0.23(-0.71,0.13)$ & 2.24 & $0.249(0.22,0.36)$ & -3.76 & 15.54 & 1.56 & 13 \\
\hline $26-012$ & 2M J04552333+30 & 0.01 & $12.6(8.3,18.6)$ & -3.00 & 0.13 & $0.013(0.01,0.01)$ & -3.65 & 10.75 & 1.55 & 2 \\
\hline $26-034$ & 2M J04554046+30 & 0.05 & $10.2(6.5,17.0)$ & -3.00 & 0.11 & $0.011(0.01,0.01)$ & -3.87 & 8.79 & 0.52 & 1 \\
\hline $26-043$ & $\mathrm{AB}$ Aur & $0.05(0.03,0.07)$ & $4.8 \quad(4.3,5.5)$ & $-1.55(-1.78,-1.41)$ & 3.80 & $0.349(0.32,0.38)$ & -5.73 & 5.70 & 1.25 & 125 \\
\hline $26-050$ & 2MJ04554757/801 & $0.10(0.06,0.15)$ & $10.8(8.3,12.3)$ & $-3.00(-3.00,-1.76)$ & 0.71 & $0.070(0.06,0.08)$ & -3.78 & 9.29 & 1.01 & 21 \\
\hline $26-067$ & SU Aur & $0.47(0.43,0.48)$ & $6.4 \quad(6.2,7.6)$ & $-1.11(-1.21,-1.06)$ & 95.36 & $9.464(8.42,9.70)$ & -3.61 & 8.89 & 1.56 & 397 \\
\hline $26-072$ & HBC 427 & $0.04(0.03,0.05)$ & $(7.8,10.8)$ & $-0.81(-1.08,-0.57)$ & 33.01 & $3.558(3.39,3.57)$ & -3.08 & 14.11 & 0.90 & 107 \\
\hline \multicolumn{11}{|c|}{ Additional sources from Chandra } \\
\hline C1-0 & KPNO-Tau 10 & & $\ldots$ & & & & & & & \\
\hline $\mathrm{C} 1-1$ & IRAS $04158+2805$ & $4.36(2.58,6.12)$ & $(2.0,31.6)$ & $0.04(-3.00,1.00)$ & 8.10 & 0.882 & -2.34 & 13.10 & 0.88 & 6 \\
\hline $\mathrm{C} 2-1$ & Haro 6-5 B & $\ldots$ & $\ldots$ & $\ldots$ & $\ldots$ & $\ldots$ & 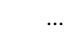 & $\ldots$ & $\ldots$ & $\ldots$ \\
\hline $\mathrm{C} 2-2$ & FS Tau AC & $1.10(0.95,1.38)$ & $(2.0,31.6)$ & $0.99 \quad(0.26,1.00)$ & 2.84 & 0.391 & -3.50 & 39.18 & 1.13 & 11 \\
\hline C3-1 & FV Tau/c AB & $\ldots$ & $\ldots$ & 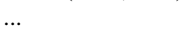 & $\cdots$ & $\ldots$ & $\ldots$ & $\ldots$ & $\ldots$ & $\ldots$ \\
\hline C3-2 & DG Tau B & $\ldots$ & $\ldots$ & $\ldots$ & $\ldots$ & $\ldots$ & $\cdots$ & ... & $\cdots$ & ... \\
\hline C4-1 & GV Tau AB & $\ldots$ & $\ldots$ & $\ldots$ & $\ldots$ & $\ldots$ & $\cdots$ & $\ldots$ & $\cdots$ & $\cdots$ \\
\hline C5-2 & HN Tau AB & $\ldots$ & $\ldots$ & $\ldots$ & $\ldots$ & ... & $\cdots$ & ... & $\cdots$ & $\ldots$ \\
\hline C5-1 & L1551 55 & $\ldots$ & $\ldots$ & $\ldots$ & ... & $\ldots$ & $\ldots$ & $\ldots$ & $\ldots$ & ... \\
\hline C5-4 & HD 28867 & $\ldots$ & $\ldots$ & $\ldots$ & $\ldots$ & $\ldots$ & $\ldots$ & $\ldots$ & $\ldots$ & $\ldots$ \\
\hline C5-3 & DM Tau & $\ldots$ & $\ldots$ & $\ldots$ & $\ldots$ & $\ldots$ & $\ldots$ & $\ldots$ & $\ldots$ & $\ldots$ \\
\hline C6-1 & CFHT-BD Tau 4 & $\ldots$ & $\ldots$ & $\ldots$ & $\ldots$ & $\cdots$ & $\ldots$ & $\cdots$ & $\ldots$ & $\ldots$ \\
\hline C6-0 & L1527 IRS & $\ldots$ & $\ldots$ & $\ldots$ & $\ldots$ & $\ldots$ & $\ldots$ & $\ldots$ & $\ldots$ & $\ldots$ \\
\hline C6-0 & CFHT-Tau 17 & $\ldots$ & $\ldots$ & $\ldots$ & $\ldots$ & $\ldots$ & $\ldots$ & $\ldots$ & $\ldots$ & $\ldots$ \\
\hline C6-2 & IRAS $04370+2559$ & $1.31(1.19,1.51)$ & $31.6(23.4,31.6)$ & -1.00 & 5.94 & 0.773 & -3.02 & 33.60 & 1.03 & 12 \\
\hline
\end{tabular}

${ }^{a} \mathrm{EM}_{t}$ is sum of EM over all DEM bins from $\log T=6.0$ to $\log T=7.9$; given in units of $10^{52} \mathrm{~cm}^{-3}$

${ }^{b} L_{\mathrm{X}}$ for $[0.3,10] \mathrm{keV}$, in units of $10^{30} \mathrm{erg} \mathrm{s}^{-1}$

Notes on individual objects:

- For several detected, faint Chandra sources, only 1-T fits were derived; see Table 6.

- Fit performed with MOS spectra for: HBC 352 (XEST-27-115), CY Tau (23-002), CY Tau (24-002), FQ Tau (23-067), 2M-J04213459 (11-023), FX Tau (13-035), V827 Tau (22-097), FZ Tau (03-023), CFHT-Tau 5 (03-031), GH Tau (04-010), V955 Tau (10-020), 2M J04552333+30, 2M J04554046+30, 2M J04554757/801, AB Aur, SU Aur, 2M J04552333+30 (26-012), 2M J04554046+30 (26034), 2M J04554757/801 (26-050), AB Aur (26-043), SU Aur (26-067).

- HP Tau/G2 and G3 = XEST-08-051 (separation: 10") were fitted as one source. The ratio of the normalizations was derived from PSF fitting in the image.

- CW Tau = XEST-20-046: fit is of low quality. CW Tau may be a two-absorber X-ray source (Güdel et al. 2007b).

- DG Tau A (XEST-02-022), GV Tau (XEST-13-004), and DP Tau (XEST-10-045) require 2-T fits with two absorbers; see Table 6.

- L1489 IRS = XEST-06-049 required $\mathrm{Fe}=0.46$ (AG89) for acceptable fit.

- HD $283572=$ XEST-21-039 required $\mathrm{Fe}=0.27$ (AG89) for acceptable fit.

- IRAS $04303+2240=$ XEST-17-027 required $\mathrm{Fe}=0.43$ (AG89) for acceptable fit.

- HL Tau = XEST-22-043 required Fe = 0.77 (AG89) for acceptable fit.

- L1551 IRS 5 = XEST-22-040: the lightly absorbed source cannot originate from heavily absorbed protostar.

- CFHT-Tau 10 = XEST-11-037: Too few counts in spectrum for reliable spectral fit. Adopted model parameters are characteristic for low-mass stars, and $N_{\mathrm{H}}$ has been derived from $A_{\mathrm{V}} . L_{\mathrm{X}}$ has been determined from count rate-to-flux conversion using spectral model in XSPEC. 
Table 6. X-ray parameters of targets in XEST (3): Plasma parameters from the 1-T and 2-T fits.

\begin{tabular}{|c|c|c|c|c|c|c|c|c|c|c|c|}
\hline XEST & Name & $\begin{array}{l}N_{\mathrm{H}} \quad(1 \sigma \text { range }) \\
\left(10^{22} \mathrm{~cm}^{-2}\right)\end{array}$ & $\begin{array}{r}T_{1}^{a} \\
(\mathrm{MK})\end{array}$ & $\begin{array}{r}T_{2} \\
(\mathrm{MK})\end{array}$ & $\begin{array}{r}\mathrm{EM}_{1}^{b} \\
\left(10^{52}\right)\end{array}$ & $\begin{array}{r}\mathrm{EM}_{2}^{b} \\
\left(10^{52}\right)\end{array}$ & $\begin{array}{r}L_{\mathrm{X}}^{c} \\
\left(10^{30}\right)\end{array}$ & $\begin{array}{r}\log \\
L_{\mathrm{X}} / L_{*}\end{array}$ & $\begin{array}{r}T_{\mathrm{av}} \\
(\mathrm{MK}) \\
\end{array}$ & $\chi_{\text {red }}^{2 d}$ & d.o.f. \\
\hline $27-115$ & HBC 352 & $0.19(0.17,0.21)$ & 7.54 & 23.77 & 10.35 & 11.52 & 2.307 & -3.09 & 13.81 & 0.87 & 128 \\
\hline $27-000$ & HBC 353 & $\ldots$ & & & $\cdots$ & & 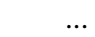 & $\ldots$ & & & \\
\hline $06-005$ & HBC 358 AB & $0.00(0.00,0.03)$ & 4.29 & 14.26 & 1.41 & 2.12 & 0.346 & -3.49 & 8.82 & 0.73 & 20 \\
\hline $06-007$ & НBC 359 & $0.00(0.00,0.02)$ & 5.33 & 15.19 & 3.06 & 3.53 & 0.628 & -3.20 & 9.34 & 1.09 & 56 \\
\hline $06-059$ & L1489 IRS & $6.54(6.08,6.88)$ & $\ldots$ & 50.20 & $\ldots$ & 26.57 & 4.003 & -3.67 & 50.20 & 1.02 & 100 \\
\hline $20-001$ & LkCa 1 & $0.07(0.00,0.21)$ & 1.39 & 8.35 & 1.76 & 0.82 & 0.205 & -3.85 & 2.46 & 1.27 & 15 \\
\hline $20-005$ & Anon 1 & $0.28(0.27,0.29)$ & 8.58 & 24.12 & 19.05 & 14.11 & 3.473 & -3.46 & 13.32 & 1.02 & 228 \\
\hline $20-000$ & IRAS $04108+2803 \mathrm{~A}$ & & $\ldots$ & & $\cdots$ & & & & & & \\
\hline $20-022$ & IRAS $04108+2803 \mathrm{~B}$ & $7.86(3.40,28.0)$ & $\ldots$ & 11.59 & $\ldots$ & 5.95 & 0.588 & -3.42 & 11.59 & 0.61 & 8 \\
\hline $20-000$ & 2M J04141188+28 & 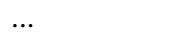 & $\ldots$ & & $\ldots$ & & & ... & 0. & & \\
\hline $20-042$ & V773 Tau ABC & $0.14(0.14,0.15)$ & 8.81 & 28.17 & 41.39 & 37.63 & 8.588 & -3.40 & 15.32 & 1.28 & 525 \\
\hline $20-043$ & FM Tau & $0.14(0.12,0.15)$ & 7.19 & 29.45 & 1.41 & 3.06 & 0.494 & -3.55 & 18.87 & 1.17 & 77 \\
\hline $20-046$ & CW Tau & $\ldots$ & $\cdots$ & $\ldots$ & $\ldots$ & $\ldots$ & & $\ldots$ & $\ldots$ & $\ldots$ & $\cdots$ \\
\hline $20-047$ & CIDA 1 & $0.34(0.04,1.10)$ & 6.38 & $\ldots$ & 0.24 & $\ldots$ & 0.031 & $x_{2}$ & 6.38 & 0.02 & 2 \\
\hline $20-056$ & MHO 2/1 & $1.01(0.84,1.15)$ & 8.00 & 28.87 & 6.35 & 10.35 & 1.853 & -3.07 & 17.72 & 0.99 & 84 \\
\hline $20-058$ & MHO 3 & $0.50(0.40,0.69)$ & 11.01 & 35.94 & 2.12 & 1.18 & 0.357 & -3.99 & 16.80 & 0.88 & 19 \\
\hline $20-069$ & FO Tau AB & $0.18(0.10,0.50)$ & & 13.80 & & 0.47 & 0.052 & -4.76 & 13.80 & 1.41 & 4 \\
\hline $20-073$ & CIDA 2 & $0.03(0.00,0.06)$ & 7.77 & $\ldots$ & 1.18 & $\ldots$ & 0.115 & -4.03 & 7.77 & 0.79 & 19 \\
\hline $23-002$ & CY Tau & $0.01(0.00,0.16)$ & 7.77 & $\ldots$ & 1.18 & $\ldots$ & 0.108 & -4.25 & 7.77 & 1.85 & 9 \\
\hline $24-002$ & CY Tau & $0.12(0.03,0.22)$ & 8.81 & $\ldots$ & 2.12 & $\ldots$ & 0.202 & -3.98 & 8.81 & 1.19 & 12 \\
\hline 23-004 & LkCa 5 & $0.03(0.01,0.04)$ & 4.75 & 17.16 & 2.35 & 2.35 & 0.440 & -3.51 & 9.03 & 0.75 & 84 \\
\hline 24-004 & LkCa 5 & $0.01(0.00,0.02)$ & 8.35 & 28.29 & 3.06 & 2.35 & 0.588 & -3.38 & 14.19 & 1.10 & 71 \\
\hline $23-008$ & CIDA 3 & $0.71(0.52,0.84)$ & $\ldots$ & 38.38 & $\ldots$ & 0.94 & 0.115 & -3.67 & 38.38 & 0.89 & 9 \\
\hline $24-008$ & CIDA 3 & $0.43(0.19,0.68)$ & $\ldots$ & 126.38 & $\ldots$ & 1.18 & 0.226 & -3.38 & 126.38 & 0.62 & 7 \\
\hline 23-015 & V410 X3 & $0.14(0.09,0.24)$ & 4.41 & 63.07 & 0.49 & 0.16 & 0.071 & -3.66 & 8.58 & 0.65 & 12 \\
\hline $24-015$ & V410 X3 & $0.00(0.00,0.07)$ & 7.88 & $\ldots$ & 0.31 & $\ldots$ & 0.031 & -4.02 & 7.88 & 0.78 & 6 \\
\hline 23-018 & V410 A13 & $0.29(0.02,0.62)$ & $=10.00$ & $\ldots$ & 0.05 & $\ldots$ & 0.006 & -4.40 & 10.00 & 0.50 & 3 \\
\hline $24-000$ & V410 A13 & $\ldots$ & $\ldots$ & $\ldots$ & $\ldots$ & $\ldots$ & $\ldots$ & $\ldots$ & $\ldots$ & $\ldots$ & $\cdots$ \\
\hline $23-000$ & V410 A24 & $\ldots$ & $\ldots$ & $\ldots$ & $\ldots$ & $\ldots$ & $\ldots$ & $\ldots$ & $\ldots$ & $\ldots$ & $\ldots$ \\
\hline $24-000$ & V410 A24 & $\ldots$ & $\ldots$ & $\ldots$ & $\ldots$ & $\ldots$ & $\ldots$ & $\ldots$ & $\ldots$ & $\ldots$ & $\ldots$ \\
\hline $23-029$ & V410 A25 & $0.71(0.60,0.80)$ & 1.28 & 22.14 & 103.48 & 6.35 & 3.807 & -3.21 & 1.51 & 1.11 & 89 \\
\hline $24-027$ & V410 A25 & $0.65(0.51,0.73)$ & 1.62 & 27.83 & 11.05 & 4.94 & 1.174 & -3.72 & 3.90 & 1.04 & 46 \\
\hline $23-032$ & V410 Tau ABC & $0.00(0.00,0.01)$ & 8.81 & 24.81 & 19.52 & 14.11 & 3.523 & -3.38 & 13.60 & 1.03 & 115 \\
\hline $24-028$ & V410 Tau ABC & $0.02(0.01,0.02)$ & 8.70 & 22.03 & 19.28 & 23.05 & 4.402 & -3.28 & 14.43 & 1.30 & 413 \\
\hline $23-033$ & DD Tau AB & $0.28(0.22,0.54)$ & 5.10 & 139.48 & 0.26 & 0.52 & 0.122 & -4.03 & 46.29 & 1.41 & 5 \\
\hline $24-029$ & DD Tau AB & $0.27(0.18,0.40)$ & $\cdots$ & 38.72 & $\cdots$ & 0.94 & 0.132 & -4.00 & 38.72 & 1.09 & 6 \\
\hline $23-035$ & CZ Tau AB & $0.17(0.14,0.21)$ & 7.42 & $\ldots$ & 2.12 & $\ldots$ & 0.205 & -3.71 & 7.42 & 0.86 & 29 \\
\hline $24-030$ & CZ Tau AB & $0.19(0.15,0.23)$ & 7.07 & 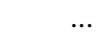 & 3.06 & $\ldots$ & 0.292 & -3.55 & 7.07 & 1.43 & 23 \\
\hline $23-036$ & IRAS $04154+2823$ & $4.10(2.49,6.38)$ & $\ldots$ & 67.83 & $\ldots$ & 1.18 & 0.176 & -3.45 & 67.83 & 0.98 & 7 \\
\hline 24-031 & IRAS $04154+2823$ & $7.67(1.15,25.8)$ & $\ldots$ & 14.14 & $\ldots$ & 2.89 & 0.282 & -3.25 & 14.14 & 0.95 & 1 \\
\hline 23-037 & V410 X2 & $1.23(1.14,1.31)$ & 7.54 & 32.81 & 16.93 & 2.12 & 1.973 & -3.77 & 8.88 & 0.81 & 83 \\
\hline $24-032$ & V410 X2 & $1.43(1.12,1.67)$ & 3.48 & 15.19 & 46.33 & 9.88 & 4.953 & -3.37 & 4.51 & 0.57 & 23 \\
\hline 23-045 & V410 X4 & $0.86(0.71,1.05)$ & $\ldots$ & 14.72 & $\ldots$ & 1.76 & 0.172 & -4.46 & 14.72 & 0.59 & 16 \\
\hline $24-038$ & V410 X4 & $0.56(0.28,1.12)$ & & 19.13 & $\ldots$ & 1.58 & 0.160 & -4.50 & 19.13 & 0.11 & 5 \\
\hline 23-047 & V892 Tau & $0.86(0.83,0.89)$ & 10.55 & 28.87 & 32.69 & 39.04 & 7.923 & -4.57 & 18.25 & 1.15 & 372 \\
\hline $24-040$ & V892 Tau & $1.25(1.12,1.40)$ & 4.87 & 22.49 & 108.65 & 35.98 & 13.779 & -4.33 & 7.13 & 0.87 & 85 \\
\hline $23-048$ & LR 1 & $0.63(0.31,1.60)$ & $\ldots$ & 277.10 & $\ldots$ & 0.24 & 0.045 & -4.60 & 277.10 & 0.30 & 3 \\
\hline $24-000$ & LR 1 & & $\ldots$ & & $\ldots$ & & & $\ldots$ & & $\ldots$ & $\ldots$ \\
\hline $23-050$ & V410 X7 & $0.77(0.64,0.91)$ & 9.97 & 18.09 & 3.06 & 3.29 & 0.626 & -3.49 & 13.58 & 0.73 & 28 \\
\hline $24-042$ & V410 X7 & $0.86(0.73,1.01)$ & 7.88 & 30.72 & 10.58 & 20.70 & 3.584 & -2.73 & 19.39 & 0.90 & 81 \\
\hline $23-000$ & V410 A20 & $\ldots$ & $\ldots$ & $\ldots$ & $\ldots$ & $\ldots$ & $\ldots$ & $\ldots$ & $\ldots$ & $\ldots$ & $\ldots$ \\
\hline $24-000$ & V410 A20 & $\ldots$ & $\ldots$ & $\ldots$ & $\ldots$ & $\ldots$ & $\ldots$ & $\ldots$ & $\ldots$ & $\ldots$ & $\ldots$ \\
\hline $23-056$ & Hubble 4 & $0.31(0.30,0.33)$ & 4.29 & 18.55 & 33.39 & 35.04 & 6.491 & -3.20 & 9.08 & 1.10 & 382 \\
\hline $24-047$ & Hubble 4 & $0.21(0.20,0.22)$ & 8.70 & 22.96 & 20.46 & 19.52 & 4.167 & -3.40 & 13.97 & 1.04 & 279 \\
\hline $23-000$ & KPNO-Tau 2 & $\ldots$ & $\ldots$ & $\cdots$ & $\cdots$ & $\cdots$ & $\cdots$ & $\cdots$ & $\cdots$ & $\ldots$ & $\cdots$ \\
\hline $24-000$ & KPNO-Tau 2 & $\ldots$ & $\ldots$ & $\ldots$ & $\ldots$ & $\ldots$ & $\ldots$ & $\ldots$ & $\ldots$ & $\ldots$ & $\ldots$ \\
\hline $23-000$ & CoKu Tau 1 & $\ldots$ & $\ldots$ & $\ldots$ & $\ldots$ & $\ldots$ & $\ldots$ & $\ldots$ & $\ldots$ & $\ldots$ & $\ldots$ \\
\hline $24-000$ & CoKu Tau 1 & $\ldots$ & $\ldots$ & $\ldots$ & $\ldots$ & $\ldots$ & $\ldots$ & $\ldots$ & $\ldots$ & $\ldots$ & $\ldots$ \\
\hline $23-061$ & V410 X6 & $0.73(0.51,0.99)$ & 3.25 & $\ldots$ & 6.35 & $\ldots$ & 0.541 & -3.15 & 3.25 & 0.64 & 7 \\
\hline
\end{tabular}


Table 6. continued.

\begin{tabular}{|c|c|c|c|c|c|c|c|c|c|c|c|}
\hline$\overline{\mathrm{XEST}}$ & Name & $\begin{array}{l}N_{\mathrm{H}} \quad(1 \sigma \text { range }) \\
\left(10^{22} \mathrm{~cm}^{-2}\right)\end{array}$ & $\begin{array}{r}T_{1}^{a} \\
(\mathrm{MK})\end{array}$ & $\begin{array}{r}T_{2} \\
(\mathrm{MK})\end{array}$ & $\begin{array}{c}\mathrm{EM}_{1}^{b} \\
\left(10^{52}\right)\end{array}$ & $\begin{array}{c}\mathrm{EM}_{2}^{b} \\
\left(10^{52}\right)\end{array}$ & $\begin{array}{r}L_{\mathrm{X}}^{c} \\
\left(10^{30}\right)\end{array}$ & $\begin{array}{r}\log \\
L_{\mathrm{X}} / L_{*}\end{array}$ & $\begin{array}{r}T_{\mathrm{av}} \\
(\mathrm{MK})\end{array}$ & $\chi_{\text {red }}^{2 d}$ & d.o.f. \\
\hline $24-054$ & V410 X6 & $0.37(0.28,0.64)$ & 7.19 & 50.20 & 1.65 & 0.47 & 0.214 & -3.56 & 11.07 & 1.21 & 8 \\
\hline $23-063$ & V410 X5 & $0.40(0.34,0.48)$ & 6.96 & 18.20 & 1.65 & 1.65 & 0.322 & -3.00 & 11.25 & 1.08 & 31 \\
\hline $24-055$ & V410 X5 & $0.81(0.75,0.85)$ & 4.64 & 55.19 & 15.05 & 3.06 & 1.823 & -2.24 & 7.05 & 0.88 & 40 \\
\hline $23-067$ & FQ Tau AB & $0.45(0.26,0.80)$ & 6.26 & $\ldots$ & 0.86 & $\ldots$ & 0.085 & -3.98 & 6.26 & 1.48 & 7 \\
\hline $24-058$ & FQ Tau AB & 0.35 & 6.84 & $\ldots$ & 0.41 & $\ldots$ & 0.040 & -4.31 & 6.84 & 0.88 & 1 \\
\hline $28-100$ & ВР Тau & $0.09(0.08,0.09)$ & 4.75 & 22.26 & 9.64 & 7.53 & 1.482 & -3.39 & 9.35 & 1.10 & 427 \\
\hline $23-074$ & V819 Tau AB & $0.21(0.20,0.23)$ & 4.64 & 17.51 & 15.52 & 9.64 & 2.356 & -3.17 & 7.72 & 1.05 & 198 \\
\hline $24-061$ & V819 Tau AB & $0.20(0.17,0.22)$ & 4.17 & 14.72 & 11.29 & 9.64 & 1.945 & -3.26 & 7.46 & 0.85 & 109 \\
\hline $16-000$ & IRAS 04166+2706 & $\ldots$ & $\cdots$ & $\cdots$ & $\cdots$ & $\cdots$ & $\cdots$ & $\ldots$ & $\cdots$ & $\cdots$ & $\ldots$ \\
\hline $16-000$ & IRAS $04169+2702$ & $\ldots$ & $\ldots$ & $\ldots$ & $\ldots$ & $\ldots$ & $\ldots$ & $\ldots$ & $\ldots$ & $\ldots$ & $\ldots$ \\
\hline $11-000$ & CFHT-Tau 19 & $\ldots$ & $\ldots$ & $\ldots$ & $\ldots$ & $\ldots$ & $\ldots$ & $\ldots$ & $\ldots$ & $\ldots$ & $\ldots$ \\
\hline $11-000$ & IRAS $04181+2655$ & $\ldots$ & $\ldots$ & $\ldots$ & $\ldots$ & $\ldots$ & $\ldots$ & $\ldots$ & $\ldots$ & $\ldots$ & $\ldots$ \\
\hline $11-000$ & IRAS $04181+2654 \mathrm{AB}$ & $\ldots$ & $\ldots$ & $\ldots$ & $\ldots$ & $\ldots$ & $\ldots$ & $\ldots$ & $\ldots$ & $\ldots$ & $\ldots$ \\
\hline $11-023$ & 2M J04213459 & $0.12(0.01,1.10)$ & 9.97 & $\ldots$ & 0.24 & $\ldots$ & 0.024 & -4.02 & 9.97 & 0.63 & 3 \\
\hline $01-028$ & IRAS 04187+1927 & $0.68(0.64,0.80)$ & 5.10 & 21.68 & 7.48 & 3.53 & 1.072 & $\ldots$ & 8.11 & 0.87 & 78 \\
\hline $11-037$ & CFHT-Tau 10 & 0.65 & $=10.00$ & $\ldots$ & 0.07 & $\ldots$ & 0.007 & -4.06 & 10.00 & 0.36 & 2 \\
\hline $11-000$ & 2M J04215450+2652 & & & & & & & & & & \\
\hline $21-038$ & RY Tau & $0.76(0.66,0.85)$ & 5.91 & 37.57 & 15.29 & 27.28 & 5.242 & -3.75 & 19.34 & 1.15 & 157 \\
\hline 21-039 & HD 283572 & $0.08(0.08,0.08)$ & 8.46 & 23.30 & 59.50 & 61.61 & 12.687 & -3.30 & 14.16 & 1.16 & 522 \\
\hline 01-045 & T Tau N(+Sab) & $0.34(0.33,0.35)$ & 4.52 & 23.65 & 33.86 & 57.85 & 9.395 & -3.56 & 12.84 & 1.36 & 626 \\
\hline $11-054$ & Haro 6-5 B & $22.0(14.4,33.6)$ & $\ldots$ & 12.17 & ... & 69.61 & 6.848 & -1.42 & 12.17 & 0.90 & 8 \\
\hline $11-057$ & FS Tau AC & $1.70(1.53,1.87)$ & 3.83 & 36.17 & 36.92 & 24.69 & 6.434 & -2.28 & 9.42 & 1.06 & 154 \\
\hline 21-044 & LkCa 21 & $0.10(0.08,0.12)$ & 4.29 & 13.22 & 4.00 & 3.53 & 0.698 & -3.53 & 7.27 & 1.13 & 81 \\
\hline $01-054$ & RX J0422.1+1934 & $0.27(0.27,0.28)$ & 8.46 & 21.91 & 10.82 & 18.11 & 3.010 & & 15.35 & 1.04 & 410 \\
\hline 01-062 & 2M J04221332+1934 & $0.40(0.17,0.65)$ & 4.64 & 9.97 & 0.63 & 0.05 & 0.059 & -3.06 & 4.89 & 1.11 & 5 \\
\hline $11-079$ & CFHT-Tau 21 & $1.48(1.09,1.96)$ & & 30.26 & & 1.41 & 0.169 & -3.94 & 30.26 & 0.69 & 7 \\
\hline $02-013$ & FV Tau AB & $1.51(0.97,2.21)$ & 3.94 & 39.65 & 8.94 & 2.82 & 1.176 & -3.59 & 6.86 & 1.04 & 12 \\
\hline $02-000$ & FV Tau/c AB & $\ldots$ & $\ldots$ & $\ldots$ & $\ldots$ & $\ldots$ & $\ldots$ & $\ldots$ & $\ldots$ & $\ldots$ & $\ldots$ \\
\hline $02-016$ & KPNO-Tau 13 & $0.30(0.22,0.42)$ & 6.03 & 21.33 & 0.82 & 0.42 & 0.122 & -3.68 & 9.26 & 0.71 & 10 \\
\hline $02-000$ & DG Tau B & $\ldots$ & & & & & & & & & $\ldots$ \\
\hline $02-022$ & DG Tau A & $0.11(0.08,0.14)$ & 3.71 & 22.96 & 0.80 & 1.69 & 0.252 & -4.41 & 12.80 & 0.74 & 33 \\
\hline $02-000$ & KPNO-Tau 4 & $\ldots$ & $\ldots$ & $\ldots$ & $\ldots$ & $\ldots$ & $\ldots$ & $\ldots$ & $\ldots$ & $\ldots$ & $\ldots$ \\
\hline $02-000$ & IRAS $04248+2612 \mathrm{AB}$ & $\ldots$ & $\ldots$ & $\ldots$ & $\ldots$ & $\ldots$ & $\ldots$ & $\ldots$ & 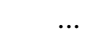 & $\ldots$ & $\ldots$ \\
\hline $15-020$ & JH 507 & $0.27(0.21,0.34)$ & 3.94 & 20.75 & 4.23 & 0.94 & 0.456 & -3.55 & 5.33 & 0.46 & 30 \\
\hline $13-004$ & GV Tau AB & $0.12(0.01,0.79)$ & 5.80 & 47.77 & 0.45 & 5.48 & 0.647 & -4.03 & 40.75 & 1.08 & 5 \\
\hline $13-000$ & IRAS $04264+2433$ & 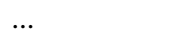 & $\ldots$ & & $\ldots$ & $\ldots$ & ... & $\ldots$ & $\ldots$ & $\ldots$ & $\ldots$ \\
\hline $15-040$ & DH Tau AB & $0.18(0.18,0.19)$ & 8.58 & 23.54 & 33.16 & 42.80 & 8.003 & -2.43 & 15.15 & 0.94 & 380 \\
\hline $15-042$ & DI Tau AB & $0.12(0.11,0.14)$ & 7.65 & 18.20 & 6.58 & 7.76 & 1.437 & -3.42 & 12.23 & 0.96 & 110 \\
\hline $15-044$ & KPNO-Tau 5 & 0.01 & 6.61 & & 0.09 & & 0.009 & -3.99 & 6.61 & 0.44 & 3 \\
\hline 14-006 & IQ Tau A & $0.41(0.33,0.52)$ & $\ldots$ & 14.26 & $\ldots$ & 3.29 & 0.313 & -4.03 & 14.26 & 1.57 & 10 \\
\hline $13-000$ & CFHT-Tau 20 & $\ldots$ & $\ldots$ & $\ldots$ & $\ldots$ & $\ldots$ & $\ldots$ & $\ldots$ & $\ldots$ & $\ldots$ & $\ldots$ \\
\hline $14-000$ & KPNO-Tau 6 & $\ldots$ & $\ldots$ & $\ldots$ & $\ldots$ & $\ldots$ & $\ldots$ & $\ldots$ & $\ldots$ & $\ldots$ & $\ldots$ \\
\hline $13-035$ & FX Tau AB & $0.20(0.14,0.29)$ & 8.46 & $\ldots$ & 0.39 & $\ldots$ & 0.393 & -4.00 & 8.46 & 0.68 & 6 \\
\hline $14-057$ & DK Tau AB & $0.31(0.28,0.34)$ & 4.17 & 18.67 & 4.70 & 6.58 & 1.061 & -3.67 & 10.00 & 0.83 & 105 \\
\hline $14-000$ & KPNO-Tau 7 & $\ldots$ & $\cdots$ & ... & $\cdots$ & $\cdots$ & $\cdots$ & & $\cdots$ & 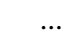 & $\ldots$ \\
\hline $22-013$ & MHO 9 & $0.20(0.12,0.28)$ & 1.04 & 5.45 & 6.11 & 1.18 & 0.235 & -3.56 & 1.36 & 0.77 & 19 \\
\hline $22-021$ & MHO 4 & $0.13(0.09,0.16)$ & 5.10 & 13.45 & 0.52 & 0.54 & 0.101 & -3.26 & 8.37 & 0.75 & 14 \\
\hline $22-040$ & L1551 IRS5 & $0.18(0.10,0.27)$ & $\ldots$ & 27.83 & $\ldots$ & 0.16 & 0.019 & -5.72 & 27.83 & 1.29 & 6 \\
\hline $22-042$ & LkHa 358 & $1.78(0.85,3.24)$ & $\ldots$ & 36.64 & $\ldots$ & 0.49 & 0.066 & -4.54 & 36.64 & 0.44 & 11 \\
\hline $22-000$ & HH 30 & $\ldots$ & $\ldots$ & & $\ldots$ & $\ldots$ & & & & & \\
\hline $22-043$ & HL Tau & $2.79(2.55,3.21)$ & 22.38 & 159.42 & 21.64 & 2.12 & 3.285 & -3.25 & 26.66 & 1.14 & 85 \\
\hline $22-047$ & XZ Tau AB & $0.24(0.21,0.27)$ & 8.70 & 26.43 & 4.00 & 3.76 & 0.849 & -3.18 & 14.91 & 1.07 & 37 \\
\hline $22-056$ & L1551 NE & $5.51(1.64,13.5)$ & $\ldots$ & 43.13 & $\ldots$ & 0.33 & 0.045 & $\ldots$ & 43.13 & 0.03 & 2 \\
\hline 03-005 & HK Tau AB & $0.89(0.40,1.90)$ & $\ldots$ & 79.54 & & 0.47 & 0.080 & -4.43 & 79.54 & 2.46 & 2 \\
\hline $22-070$ & V710 Tau BA & $0.23(0.22,0.25)$ & 7.77 & 18.32 & 6.82 & 4.94 & 1.166 & -3.56 & 11.14 & 0.94 & 157 \\
\hline 19-009 & JH 665 & $0.13(0.02,0.27)$ & 6.84 & & 0.71 & $\ldots$ & 0.059 & -4.23 & 6.84 & 0.99 & 4 \\
\hline $22-089$ & L1551 51 & $0.08(0.07,0.09)$ & 7.30 & 16.81 & 9.88 & 6.35 & 1.611 & -3.05 & 10.12 & 1.14 & 203 \\
\hline $22-097$ & V827 Tau & $0.05(0.04,0.05)$ & 8.35 & 22.38 & 13.64 & 21.64 & 3.702 & -3.06 & 15.29 & 1.16 & 272 \\
\hline 03-016 & Haro 6-13 & $0.54(0.41,0.72)$ & $\ldots$ & 27.25 & $\ldots$ & 1.29 & 0.151 & -4.73 & 27.25 & 0.78 & 8 \\
\hline
\end{tabular}


Table 6. continued.

\begin{tabular}{|c|c|c|c|c|c|c|c|c|c|c|c|}
\hline XEST & Name & $\begin{array}{l}N_{\mathrm{H}}(1 \sigma \text { range }) \\
\left(10^{22} \mathrm{~cm}^{-2}\right)\end{array}$ & $\begin{array}{r}T_{1}^{a} \\
(\mathrm{MK})\end{array}$ & $\begin{array}{r}T_{2} \\
(\mathrm{MK})\end{array}$ & $\begin{array}{r}\mathrm{EM}_{1}^{b} \\
\left(10^{52}\right)\end{array}$ & $\begin{array}{r}\mathrm{EM}_{2}^{b} \\
\left(10^{52}\right)\end{array}$ & $\begin{array}{r}L_{\mathrm{X}}^{c} \\
\left(10^{30}\right)\end{array}$ & $\begin{array}{r}\log \\
L_{\mathrm{X}} / L_{*}\end{array}$ & $\begin{array}{r}T_{\mathrm{av}} \\
(\mathrm{MK})\end{array}$ & $\chi_{\text {red }}^{2 d}$ & d.o.f. \\
\hline $22-100$ & V826 Tau & $0.06(0.06,0.06)$ & 7.54 & 17.97 & 19.52 & 22.34 & 4.210 & -2.93 & 11.99 & 1.37 & $\overline{373}$ \\
\hline $22-101$ & MHO 5 & $0.09(0.06,0.13)$ & 2.55 & 8.46 & 0.28 & 0.80 & 0.108 & -3.59 & 6.19 & 1.06 & 30 \\
\hline 03-017 & CFHT-Tau 7 & $0.47(0.24,1.46)$ & 3.13 & $\ldots$ & 2.12 & $\ldots$ & 0.176 & -3.13 & 3.13 & 0.80 & 3 \\
\hline 03-019 & V928 Tau AB & $0.40(0.33,0.46)$ & 4.64 & 20.99 & 9.17 & 2.59 & 1.096 & -3.69 & 6.47 & 0.92 & 45 \\
\hline 03-022 & FY Tau & $0.30(0.27,0.34)$ & 9.28 & 27.94 & 2.35 & 4.47 & 0.769 & -3.70 & 19.11 & 0.99 & 33 \\
\hline 03-023 & FZ Tau & $0.28(0.22,0.47)$ & $\ldots$ & 11.48 & $\ldots$ & 2.70 & 0.268 & -4.15 & 11.48 & 0.83 & 17 \\
\hline $17-002$ & IRAS $04295+2251$ & $3.43(2.67,4.59)$ & $\ldots$ & 20.17 & $\ldots$ & 9.88 & 1.025 & -3.65 & 20.17 & 0.33 & 8 \\
\hline 19-049 & UZ Tau E+W(AB) & $0.41(0.33,0.44)$ & 3.83 & 19.01 & 4.94 & 3.06 & 0.736 & -3.67 & 7.07 & 0.77 & 60 \\
\hline $17-009$ & JH 112 & $0.90(0.68,1.02)$ & 4.06 & 30.26 & 13.17 & 2.12 & 1.399 & -3.31 & 5.36 & 0.55 & 21 \\
\hline 03-031 & CFHT-Tau 5 & $1.51(0.88,2.29)$ & 4.41 & & 10.82 & & 0.962 & -2.48 & 4.41 & 0.88 & 4 \\
\hline $04-003$ & CFHT-Tau 5 & $0.33(0.21,0.55)$ & $\ldots$ & 19.83 & $\ldots$ & 1.18 & 0.115 & -3.40 & 19.83 & 0.51 & 6 \\
\hline 03-035 & MHO 8 & $0.29(0.18,0.58)$ & 4.99 & $\ldots$ & 0.87 & $\ldots$ & 0.080 & -3.91 & 4.99 & 0.54 & 3 \\
\hline 04-009 & MHO 8 & $0.72(0.50,0.97)$ & 3.25 & $\ldots$ & 4.42 & $\ldots$ & 0.376 & -3.24 & 3.25 & 0.50 & 6 \\
\hline 04-010 & GH Tau AB & $0.11(0.06,0.19)$ & & 12.29 & & 1.20 & 0.120 & -4.41 & 12.29 & 0.78 & 6 \\
\hline 04-012 & V807 Tau SNab & $0.10(0.09,0.12)$ & 4.52 & 17.04 & 6.82 & 4.23 & 1.030 & -3.89 & 7.51 & 1.22 & 83 \\
\hline $18-004$ & KPNO-Tau 14 & $0.63(0.59,0.70)$ & $\ldots$ & 22.84 & $\ldots$ & 5.88 & 0.647 & -2.82 & 22.84 & 1.15 & 28 \\
\hline $17-000$ & CFHT-Tau 12 & & $\ldots$ & & $\ldots$ & & & $\ldots$ & 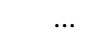 & $\ldots$ & $\ldots$ \\
\hline 04-016 & V830 Tau & $0.04(0.04,0.04)$ & 8.12 & 22.49 & 15.05 & 30.57 & 4.807 & -2.80 & 16.07 & 1.24 & 308 \\
\hline $18-000$ & IRAS S04301+261 & $\ldots$ & $\ldots$ & $\ldots$ & $\ldots$ & $\ldots$ & $\ldots$ & $\ldots$ & $\ldots$ & $\ldots$ & $\ldots$ \\
\hline $17-000$ & IRAS 04302 & $\ldots$ & $\ldots$ & $\ldots$ & $\ldots$ & $\ldots$ & $\ldots$ & $\ldots$ & $\ldots$ & $\ldots$ & $\ldots$ \\
\hline $17-027$ & IRAS $04303+2240$ & $1.59(1.41,1.78)$ & 4.06 & 39.19 & 32.22 & 19.99 & 5.548 & -3.18 & 9.67 & 0.89 & 114 \\
\hline 04-034 & GI Tau & $0.41(0.36,0.47)$ & 4.87 & 32.58 & 4.23 & 3.76 & 0.872 & -3.64 & 11.91 & 0.75 & 48 \\
\hline 04-035 & GK Tau AB & $0.40(0.37,0.55)$ & 8.81 & 28.87 & 7.29 & 4.47 & 1.244 & -3.64 & 13.83 & 1.04 & 79 \\
\hline $18-019$ & IS Tau AB & $0.65(0.50,0.80)$ & 4.41 & 17.86 & 9.41 & 4.70 & 1.310 & -3.29 & 7.03 & 1.08 & 55 \\
\hline $17-058$ & CI Tau & $0.62(0.31,1.10)$ & 7.88 & 43.71 & 0.94 & 1.65 & 0.329 & -4.01 & 23.44 & 0.52 & 10 \\
\hline $18-030$ & IT Tau AB & $0.68(0.65,0.71)$ & 11.71 & 37.33 & 11.76 & 38.57 & 6.307 & -3.17 & 28.47 & 0.90 & 426 \\
\hline $17-066$ & JH 108 & $0.17(0.15,0.21)$ & 8.81 & 24.93 & 4.70 & 5.64 & 1.115 & -3.02 & 15.54 & 1.07 & 36 \\
\hline $17-068$ & CFHT-BD Tau 1 & $0.65(0.44,0.80)$ & $\ldots$ & 15.77 & $\ldots$ & 1.88 & 0.174 & -2.58 & 15.77 & 1.38 & 2 \\
\hline $25-026$ & AA Tau & $0.94(0.84,1.03)$ & & 27.13 & & 8.94 & 1.039 & -3.47 & 27.13 & 0.82 & 43 \\
\hline 09-010 & HO Tau AB & $0.64(0.02,1.48)$ & 3.25 & & 0.47 & $\ldots$ & 0.289 & -3.36 & 3.25 & 0.36 & 3 \\
\hline 08-019 & FF Tau AB & $0.27(0.21,0.28)$ & 7.42 & 15.07 & 3.53 & 3.29 & 0.658 & -3.61 & 10.45 & 1.13 & 58 \\
\hline $12-040$ & DN Tau & $0.06(0.05,0.06)$ & 8.81 & 23.42 & 5.17 & 5.17 & 1.072 & -3.56 & 14.36 & 1.14 & 226 \\
\hline $12-000$ & IRAS $04325+2402 \mathrm{AB}$ & & & & & & & & $\ldots$ & & \\
\hline $12-059$ & CoKu Tau 3 AB & $0.50(0.48,0.52)$ & 4.41 & 18.67 & 41.86 & 43.98 & 8.207 & -2.66 & 9.24 & 1.01 & 387 \\
\hline 09-022 & KPNO-Tau 8 & $0.11(0.09,0.13)$ & 4.41 & 20.99 & 1.65 & 3.06 & 0.459 & -2.25 & 12.16 & 0.80 & 58 \\
\hline 08-037 & HQ Tau AB & $0.35(0.32,0.37)$ & 8.70 & 24.58 & 11.99 & 8.00 & 2.074 & $\ldots$ & 13.18 & 1.11 & 134 \\
\hline 09-026 & HQ Tau AB & $0.66(0.62,0.70)$ & 4.75 & 24.23 & 75.72 & 34.81 & 10.724 & $\ldots$ & 7.93 & 0.83 & 165 \\
\hline 08-043 & KPNO-Tau 15 & $0.38(0.37,0.41)$ & 8.81 & 33.51 & 5.64 & 15.52 & 2.526 & -2.33 & 23.47 & 0.92 & 260 \\
\hline 09-031 & KPNO-Tau 15 & $0.39(0.25,0.55)$ & 5.68 & 24.58 & 3.29 & 2.12 & 0.553 & -2.99 & 10.08 & 1.22 & 10 \\
\hline 08-000 & KPNO-T: & $\ldots$ & $\ldots$ & $\ldots$ & $\ldots$ & $\ldots$ & $\ldots$ & $\ldots$ & $\ldots$ & $\ldots$ & $\ldots$ \\
\hline 09-000 & KPNO & & $\ldots$ & $\ldots$ & $\ldots$ & $\ldots$ & $\ldots$ & $\ldots$ & $\ldots$ & $\ldots$ & $\ldots$ \\
\hline 08-048 & HP Tau AB & $0.48(0.45,0.51)$ & 8.58 & 23.88 & 10.11 & 10.58 & 2.187 & -3.39 & 14.48 & 1.08 & 128 \\
\hline $08-051 \mathrm{a}$ & HP Tau/G3 AB & $0.53(0.52,0.56)$ & 4.64 & 20.64 & 12.46 & 6.82 & 1.839 & -3.17 & 7.87 & 1.08 & 381 \\
\hline 08-051 & HP Tau/G2 & $0.53(0.52,0.56)$ & 4.64 & 20.64 & 93.83 & 51.27 & 13.830 & -3.26 & 7.86 & 1.08 & 381 \\
\hline 08-058 & Haro 6-28 AB & $0.34(0.29,0.45)$ & $\ldots$ & 10.55 & $\ldots$ & 2.12 & 0.202 & -3.36 & 10.55 & 0.87 & 33 \\
\hline 08-000 & CFHT-BD Tau 2 & $\ldots$ & $\ldots$ & $\ldots$ & $\ldots$ & $\ldots$ & $\ldots$ & $\ldots$ & $\ldots$ & $\ldots$ & $\ldots$ \\
\hline 08-080 & CFHT-BD Tau 3 & $0.11(0.00,0.41)$ & $=10.00$ & $\ldots$ & 0.09 & $\ldots$ & 0.009 & -3.49 & 10.00 & 0.12 & 1 \\
\hline 05-005 & CFHT-Tau 6 & $0.32(0.18,0.46)$ & 9.97 & $\ldots$ & 0.40 & $\ldots$ & 0.040 & -3.36 & 9.97 & 1.59 & 2 \\
\hline $05-000$ & IRAS $04361+2547$ & $\ldots$ & $\ldots$ & $\ldots$ & $\ldots$ & $\ldots$ & $\ldots$ & $\ldots$ & $\ldots$ & & $\ldots$ \\
\hline 05-013 & GN Tau AB & $42(1.28,3.33)$ & $\ldots$ & 14.38 & $\ldots$ & 4.23 & 0.423 & -3.82 & 14.38 & 1.60 & 5 \\
\hline 05-017 & IRAS $04365+2535$ & $24.3(9.76,63.0)$ & $\ldots$ & 49.16 & $\ldots$ & 3.29 & 0.499 & -4.23 & 49.16 & 0.48 & 1 \\
\hline 05-024 & IRAS 04369+2539 & $6.15(4.93,7.81)$ & $\ldots$ & 32.70 & $\ldots$ & 10.35 & 1.315 & -4.78 & 32.70 & 1.17 & 7 \\
\hline 07-011 & JH 223 & $0.09(0.05,0.14)$ & 8.70 & $\ldots$ & 0.71 & $\ldots$ & 0.061 & -4.06 & 8.70 & 1.04 & 10 \\
\hline 07-022 & Haro 6-32 & $0.04(0.00,0.08)$ & $\ldots$ & 11.25 & $\ldots$ & 0.94 & 0.085 & -3.74 & 11.25 & 0.47 & 6 \\
\hline 07-000 & ITG 33 A & $\ldots$ & $\ldots$ & $\ldots$ & $\ldots$ & $\ldots$ & $\ldots$ & $\ldots$ & $\ldots$ & $\ldots$ & $\ldots$ \\
\hline 07-000 & CFHT-Tau 8 & $\ldots$ & $\ldots$ & $\ldots$ & $\cdots$ & $\ldots$ & $\ldots$ & $\ldots$ & $\ldots$ & $\ldots$ & $\ldots$ \\
\hline 07-000 & IRAS $04381+2540$ & $\ldots$ & $\ldots$ & $\ldots$ & $\ldots$ & $\ldots$ & $\ldots$ & $\ldots$ & $\ldots$ & $\ldots$ & $\ldots$ \\
\hline 07-041 & IRAS $04385+2550 \mathrm{AB}$ & $0.76(0.60,0.97)$ & $\ldots$ & 32.12 & $\ldots$ & 2.82 & 0.367 & -3.28 & 32.12 & 0.70 & 9 \\
\hline $10-017$ & CoKuLk332/G2 AB & $0.63(0.59,0.67)$ & 4.75 & 22.96 & 33.63 & 11.52 & 4.313 & -2.99 & 7.10 & 0.82 & 162 \\
\hline
\end{tabular}


Table 6. continued.

\begin{tabular}{|c|c|c|c|c|c|c|c|c|c|c|c|}
\hline XEST & Name & $\begin{array}{l}N_{\mathrm{H}}(1 \sigma \text { range }) \\
\left(10^{22} \mathrm{~cm}^{-2}\right)\end{array}$ & $\begin{array}{r}T_{1}^{a} \\
(\mathrm{MK})\end{array}$ & $\begin{array}{r}T_{2} \\
(\mathrm{MK})\end{array}$ & $\begin{array}{r}\mathrm{EM}_{1}^{b} \\
\left(10^{52}\right)\end{array}$ & $\begin{array}{r}\mathrm{EM}_{2}^{b} \\
\left(10^{52}\right)\end{array}$ & $\begin{array}{r}L_{\mathrm{X}}^{c} \\
\left(10^{30}\right)\end{array}$ & $\begin{array}{r}\log \\
L_{\mathrm{X}} / L_{*}\end{array}$ & $\begin{array}{r}T_{\mathrm{av}} \\
(\mathrm{MK})\end{array}$ & $\chi_{\text {red }}^{2 d}$ & d.o.f. \\
\hline 10-018 & CoKuLk332/G1 AB & $0.23(0.15,0.31)$ & 11.48 & 43.01 & 1.88 & 1.65 & 0.414 & -4.20 & 21.26 & 0.64 & 20 \\
\hline $10-020$ & V955 Tau AB & $1.09(0.83,1.34)$ & 4.17 & 25.86 & 27.99 & 10.11 & 3.617 & -3.03 & 6.77 & 1.00 & 33 \\
\hline $10-034$ & CIDA 7 & $0.88(0.58,1.11)$ & 2.20 & $\ldots$ & 11.52 & & 0.884 & -2.34 & 2.20 & 0.58 & 6 \\
\hline $10-045$ & DP Tau & $0.00(0.00,1.05)$ & 3.25 & 43.83 & 0.05 & 0.61 & 0.101 & -3.88 & 36.40 & 1.27 & 7 \\
\hline $10-060$ & GO Tau & $0.35(0.26,0.55)$ & 6.26 & 36.29 & 1.18 & 1.18 & 0.308 & -3.67 & 15.07 & 1.53 & 12 \\
\hline $26-012$ & 2M J04552333+30 & $0.07(0.00,0.24)$ & $=10.00$ & $\ldots$ & 0.15 & $\ldots$ & 0.015 & -3.59 & 10.00 & 1.07 & 2 \\
\hline $26-034$ & 2M J04554046+30 & $0.04(0.00,0.18)$ & $=10.00$ & $\ldots$ & 0.10 & $\ldots$ & 0.010 & -3.91 & 10.00 & 0.17 & 1 \\
\hline $26-043$ & AB Aur & $0.06(0.04,0.07)$ & 2.43 & 7.54 & 1.18 & 2.59 & 0.353 & -5.73 & 5.29 & 1.36 & 124 \\
\hline $26-050$ & 2MJ04554757/801 & $0.06(0.02,0.12)$ & 8.58 & 12.17 & 0.24 & 0.24 & 0.056 & -3.88 & 10.22 & 1.03 & 20 \\
\hline $26-067$ & SU Aur & $0.56(0.54,0.60)$ & 5.22 & 23.30 & 87.01 & 32.45 & 11.641 & -3.52 & 7.84 & 1.68 & 396 \\
\hline 26-072 & HBC 427 & $0.02(0.01,0.03)$ & 9.04 & 28.06 & 14.98 & 15.07 & 3.276 & -3.11 & 15.96 & 0.92 & 106 \\
\hline \multicolumn{12}{|c|}{ Additional sources from Chandra } \\
\hline$\overline{\mathrm{C} 1-0}$ & KPNO-Tau 10 & $\ldots$ & $\cdots$ & & $\cdots$ & .. & & & & & $\cdots$ \\
\hline C1-1 & IRAS 04158+2805 & $3.24(1.95,5.47)$ & $\ldots$ & 70.72 & $\ldots$ & 2.59 & 0.414 & -2.67 & 70.72 & 0.70 & 7 \\
\hline C2-1 & Haro 6-5 B & $26.4(10.4,37.2)$ & $\ldots$ & 15.54 & $\ldots$ & 43.27 & 3.774 & -1.68 & 15.54 & $142.3 \mathrm{C}$ & 512 \\
\hline C2-2 & FS Tau AC & $0.82(0.58,1.05)$ & $\ldots$ & 70.96 & $\ldots$ & 1.95 & 0.329 & -3.57 & 70.96 & 1.02 & 12 \\
\hline C3-1 & FV Tau/c AB & $10.8(5.70,16.4)$ & $\ldots$ & 16.46 & $\ldots$ & 4.94 & 0.430 & -3.27 & 16.46 & $96.3 \mathrm{C}$ & 512 \\
\hline C3-2 & DG Tau B & $51.3(11.0,63.0)$ & $\ldots$ & 11.59 & $\ldots$ & 111.94 & 9.689 & -3.34 & 11.59 & $70.20 \mathrm{C}$ & 512 \\
\hline C4-1 & GV Tau AB & .. & $\cdots$ & $\cdots$ & $\cdots$ & $\cdots$ & & & & $\ldots$ & $\cdots$ \\
\hline C5-2 & HN Tau AB & 0.20 & $=10.00$ & $\ldots$ & $\ldots$ & $\ldots$ & 0.153 & -3.80 & 10.00 & $\ldots$ & $\ldots$ \\
\hline C5-1 & L1551 55 & 0.14 & $=10.00$ & $\cdots$ & $\ldots$ & $\ldots$ & 0.597 & -3.38 & 10.00 & $\ldots$ & $\ldots$ \\
\hline C5-4 & HD 28867 & 0.05 & $=10.00$ & $\cdots$ & $\ldots$ & $\ldots$ & 6.867 & -4.85 & 10.00 & $\ldots$ & $\ldots$ \\
\hline C5-3 & DM Tau & 0.12 & $=10.00$ & $\ldots$ & $\ldots$ & $\ldots$ & 0.181 & -3.80 & 10.00 & $\ldots$ & $\ldots$ \\
\hline C6-1 & CFHT-BD Tau 4 & 0.52 & $\ldots$ & 14.26 & $\ldots$ & 0.94 & 0.096 & -3.40 & 14.26 & 0.087 & 3 \\
\hline C6-0 & L1527 IRS & $\cdots$ & $\ldots$ & $\cdots$ & $\cdots$ & $\cdots$ & $\ldots$ & $\cdots$ & $\cdots$ & $\cdots$ & $\cdots$ \\
\hline C6-0 & CFHT-Tau 17 & $\ldots$ & $\ldots$ & $\ldots$ & $\ldots$ & $\ldots$ & $\ldots$ & $\ldots$ & $\ldots$ & $\ldots$ & $\ldots$ \\
\hline C6-2 & IRAS $04370+2559$ & $1.09(0.90,1.33)$ & $\ldots$ & 50.55 & $\ldots$ & 4.47 & 0.666 & -3.08 & 50.55 & 0.97 & 12 \\
\hline
\end{tabular}

a "=" sign before number indicates that parameter was fixed

${ }^{b} \mathrm{EM}$ in units of $10^{52} \mathrm{~cm}^{-3}$

${ }^{c} L_{\mathrm{X}}$ for $[0.3,10] \mathrm{keV}$, in units of $10^{30} \mathrm{erg} \mathrm{s}^{-1}$

${ }^{d}$ Numbers followed by 'C' denote C statistic (for low-background Chandra data)

Notes on individual objects:

- Fit performed for MOS1 + MOS2 spectra for: HBC 352 (XEST-27-115), CY Tau (23-002), CY Tau (24-002), FQ Tau (23-067), 2M-J04213459 (11-023), FX Tau (13-035), V827 Tau (22-097), FZ Tau (03-023), CFHT-Tau 5 (03-031), GH Tau (04-010), V955 Tau (10-020), 2M J04552333+30 (26-012), 2M J04554046+30 (26-034), 2M J04554757/801 (26-050), AB Aur (26-043), and SU Aur (26-067).

- HN Tau A (C5-2), L1551 55 (C5-1), DM Tau (C5-3), and HD 28867 (C5-4): T = 10 MK was adopted for Chandra HRC observations, and $N_{\mathrm{H}}$ was derived from $A_{\mathrm{V}}$ assuming standard gas-to-dust ratios.

- DG Tau A (XEST-02-022), GV Tau (XEST-13-004), and DP Tau (XEST-10-045) require different $N_{\mathrm{H}}$ for the two components (also CW Tau; see Güdel et al. 2007b). $N_{\mathrm{H}}$ for cooler component is given. For hotter component: $N_{\mathrm{H}}=1.8,4.1$, and 3.8, respectively.

- CW Tau = XEST-20-046: spectrum cannot be reliably fitted. CW Tau may be two-absorber X-ray source (Güdel et al. 2007b).

- HP Tau/G2 and G3 = XEST-08-051 (separation: 10") were fitted as one source. The ratio of the normalizations was derived from PSF fitting in the image.

- BP Tau = XEST-28-100: for an acceptable fit, two abundances were adjusted: $\mathrm{O}=0.267, \mathrm{Fe}=0.10$ (AG89).

- LkCa 1 = XEST-20-001: for an acceptable fit, Fe was adjusted: $\mathrm{Fe}=0.57$ (AG89).

- HL Tau = XEST-22-043: for an acceptable fit, Fe was adjusted: Fe = 1.06 (AG89).

- L1551 IRS 5 = XEST-22-040: the lightly absorbed source cannot originate from heavily absorbed protostar. 
Table 7. Fundamental parameters of targets in XEST (1): Names and coordinates.

\begin{tabular}{|c|c|c|c|c|c|c|}
\hline XEST & Name & $2 \mathrm{MASS}^{a}$ & $\mathrm{IRAS}^{b}$ & Alternative names & $\begin{array}{l}\mathrm{RA}(\mathrm{J} 2000.0)^{c} \\
\mathrm{~h} \mathrm{~m} \mathrm{~s}\end{array}$ & $\begin{array}{l}\overline{D e c}(\mathrm{~J} 2000.0)^{c} \\
\operatorname{deg},{ }^{\prime} \prime\end{array}$ \\
\hline $27-115$ & HBC 352 & $03542950+3203013$ & $\ldots$ & NTTS 035120+3154SW & 35429.51 & 320301.4 \\
\hline $27-000$ & HBC 353 & $03543017+3203043$ & $\ldots$ & NTTS 035120+3154NE & 35430.17 & 320304.3 \\
\hline 06-005 & HBC 358 AB & $04034930+2610520$ & $\ldots$ & NTTS 040047+2603W & 40349.31 & 261052.0 \\
\hline 06-007 & HBC 359 & $04035084+2610531$ & $\ldots$ & TTS 040047+2603 & 40350.84 & 261053.2 \\
\hline 06-059 & L1489 IRS & $04044307+2618563$ & $04016+2610$ & $\ldots$ & 40443.07 & 261856.4 \\
\hline $20-001$ & LkCa 1 & $04131414+2819108$ & $\ldots$ & HBC 365, V1095 Tau, JH 141 & 41314.14 & 281910.8 \\
\hline $20-005$ & Anon 1 & $04132722+2816247$ & & HBC 366, V1096 Tau & 41327.23 & 281624.8 \\
\hline $20-000$ & IRAS $04108+2803$ A & $04135328+2811233$ & $04108+2803 \mathrm{~A}$ & L1495N IRS & 41353.29 & 281123.4 \\
\hline $20-022$ & IRAS 04108+2803 B & $04135471+2811328$ & $04108+2803$ & $\ldots$ & 41354.72 & 281132.9 \\
\hline $20-000$ & 2M J04141188+28 & $04141188+2811535$ & & $\ldots$ & 41411.88 & 281153.5 \\
\hline $20-042$ & V773 Tau ABC & $04141291+2812124$ & $04111+2804$ & HBC 367, HD 283447 & 41412.92 & 281212.4 \\
\hline $20-043$ & FM Tau & $04141358+2812492$ & $\ldots$ & HBC 23, Haro 6-1 & 41413.58 & 281249.2 \\
\hline $20-046$ & CW Tau & $04141700+2810578$ & $04112+2803$ & HBC 25 & 41417.00 & 281057.8 \\
\hline $20-047$ & CIDA 1 & $04141760+2806096$ & & C4101 & 41417.61 & 280609.7 \\
\hline $20-056$ & MHO 2/1 & $04142639+2805597$ & $04113+2758$ & $\ldots$ & 41426.40 & 280559.7 \\
\hline $20-058$ & MHO 3 & $04143054+2805147$ & $04114+2757$ & $\ldots$ & 41430.55 & 280514.7 \\
\hline $20-069$ & FO Tau AB & $04144928+2812305$ & $04117+2804$ & HBC 369 & 41449.29 & 281230.6 \\
\hline $20-073$ & CIDA 2 & $04150515+2808462$ & $\ldots$ & C3601 & 41505.16 & 280846.2 \\
\hline $23-002$ & CY Tau & $04173372+2820468$ & C04144+2813 & HBC 28 & 41733.73 & 282046.9 \\
\hline $24-002$ & CY Tau & $04173372+2820468$ & $\mathrm{C} 04144+2813$ & HBC 28 & 41733.73 & 282046.9 \\
\hline 23-004 & LkCa 5 & $04173893+2833005$ & $\ldots$ & HBC 371, JH 153 & 41738.94 & 283300.5 \\
\hline 24-004 & LkCa 5 & $04173893+2833005$ & $\ldots$ & HBC 371, JH 153 & 41738.94 & 283300.5 \\
\hline $23-008$ & CIDA 3 & $04174965+2829362$ & $\ldots$ & C2701, V410 X1, Kim3-76 & 41749.65 & 282936.3 \\
\hline 24-008 & CIDA 3 & $04174965+2829362$ & $\ldots$ & C2701, V410 X1, Kim3-76 & 41749.65 & 282936.3 \\
\hline 23-015 & V410 X3 & $04180796+2826036$ & $\ldots$ & $\ldots$ & 41807.96 & 282603.7 \\
\hline 24-015 & V410 X3 & $04180796+2826036$ & $\ldots$ & $\ldots$ & 41807.96 & 282603.7 \\
\hline $23-018$ & V410 A13 & $04181710+2828419$ & $\ldots$ & MHO 10 & 41817.11 & 282841.9 \\
\hline $24-000$ & V410 A13 & $04181710+2828419$ & $\ldots$ & MHO 10 & 41817.11 & 282841.9 \\
\hline $23-000$ & V410 A24 & $04182239+2824375$ & $\ldots$ & $\ldots$ & 41822.39 & 282437.6 \\
\hline $24-000$ & V410 A24 & $04182239+2824375$ & $\ldots$ & $\ldots$ & 41822.39 & 282437.6 \\
\hline $23-029$ & V410 A25 & $04182909+2826191$ & $\ldots$ & $\ldots$ & 41829.10 & 282619.1 \\
\hline $24-027$ & V410 A25 & $04182909+2826191$ & $\ldots$ & $\ldots$ & 41829.10 & 282619.1 \\
\hline $23-032$ & V410 Tau ABC & $04183110+2827162$ & $\mathrm{C} 04152+2820$ & HBC 29, HD 283518 & 41831.10 & 282716.2 \\
\hline $24-028$ & V410 Tau ABC & $04183110+2827162$ & $\mathrm{C} 04152+2820$ & HBC 29, HD 283518 & 41831.10 & 282716.2 \\
\hline $23-033$ & DD Tau AB & $04183112+2816290$ & $04154+2809$ & HBC 30 & 41831.13 & 281629.0 \\
\hline 24-029 & DD Tau AB & $04183112+2816290$ & $04154+2809$ & HBC 30 & 41831.13 & 281629.0 \\
\hline $23-035$ & CZ Tau AB & $04183158+2816585$ & $\ldots$ & HBC 31 & 41831.59 & 281658.5 \\
\hline 24-030 & CZ Tau AB & $04183158+2816585$ & $\ldots$ & HBC 31 & 41831.59 & 281658.5 \\
\hline 23-036 & IRAS $04154+2823$ & $04183203+2831153$ & $04154+2823$ & $\ldots$ & 41832.03 & 283115.4 \\
\hline $24-031$ & IRAS $04154+2823$ & $04183203+2831153$ & $04154+2823$ & $\ldots$ & 41832.03 & 283115.4 \\
\hline $23-037$ & V410 X2 & $04183444+2830302$ & $\ldots$ & $\ldots$ & 41834.45 & 283030.2 \\
\hline $24-032$ & V410 X2 & $04183444+2830302$ & $\ldots$ & $\ldots$ & 41834.45 & 283030.2 \\
\hline $23-045$ & V410 X4 & $04184023+2824245$ & $\ldots$ & $\ldots$ & 41840.23 & 282424.5 \\
\hline $24-038$ & V410 X4 & $04184023+2824245$ & & $\ldots$ & 41840.23 & 282424.5 \\
\hline 23-047 & V892 Tau & $04184061+2819155$ & $04155+2812$ & HBC 373, Elias 1 & 41840.62 & 281915.5 \\
\hline $24-040$ & V892 Tau & $04184061+2819155$ & $04155+2812$ & HBC 373, Elias 1 & 41840.62 & 281915.5 \\
\hline $23-048$ & LR 1 & $04184133+2827250$ & $\ldots$ & $\ldots$ & 41841.33 & 282725.0 \\
\hline $24-000$ & LR 1 & $04184133+2827250$ & $\ldots$ & $\ldots$ & 41841.33 & 282725.0 \\
\hline $23-050$ & V410 X7 & $04184250+2818498$ & $\ldots$ & MHO-11 & 41842.50 & 281849.8 \\
\hline 24-042 & V410 X7 & $04184250+2818498$ & $\ldots$ & MHO-11 & 41842.50 & 281849.8 \\
\hline 23-000 & V410 A20 & $04184505+2820528$ & $\ldots$ & $\ldots$ & 41845.06 & 282052.8 \\
\hline $24-000$ & V410 A20 & $04184505+2820528$ & $\ldots$ & $\ldots$ & 41845.06 & 282052.8 \\
\hline $23-056$ & Hubble 4 & $04184703+2820073$ & $04157+2813$ & V1023 Tau, HBC 374 & 41847.04 & 282007.3 \\
\hline 24-047 & Hubble 4 & $04184703+2820073$ & $04157+2813$ & V1023 Tau, HBC 374 & 41847.04 & 282007.3 \\
\hline $23-000$ & KPNO-Tau 2 & $04185115+2814332$ & $\ldots$ & $\ldots$ & 41851.16 & 281433.2 \\
\hline $24-000$ & KPNO-Tau 2 & $04185115+2814332$ & $\ldots$ & $\ldots$ & 41851.16 & 281433.2 \\
\hline $23-000$ & CoKu Tau 1 & $04185147+2820264$ & $\ldots$ & HBC 375 & 41851.48 & 282026.5 \\
\hline $24-000$ & CoKu Tau 1 & $04185147+2820264$ & $\ldots$ & HBC 375 & 41851.48 & 282026.5 \\
\hline $23-061$ & V410 X6 & $04190110+2819420$ & $\ldots$ & Kim3-89 & 41901.11 & 281942.0 \\
\hline
\end{tabular}


Table 7. continued.

\begin{tabular}{|c|c|c|c|c|c|c|}
\hline XEST & Name & $2 \mathrm{MASS}^{a}$ & $\mathrm{IRAS}^{b}$ & Alternative names & $\begin{array}{l}\mathrm{RA}(\mathrm{J} 2000.0)^{c} \\
\mathrm{~h} \mathrm{~m} \mathrm{~s}\end{array}$ & $\begin{array}{l}\operatorname{Dec}(\mathbf{J} 2000.0)^{c} \\
\operatorname{deg} \prime^{\prime} \prime \prime\end{array}$ \\
\hline $24-054$ & V410 X6 & $04190110+2819420$ & $\ldots$ & Kim3-89 & 41901.11 & 281942.0 \\
\hline $23-063$ & V410 X5 & $04190197+2822332$ & $\ldots$ & MHO-12 & 41901.98 & 282233.2 \\
\hline $24-055$ & V410 X5 & $04190197+2822332$ & $\ldots$ & MHO-12 & 41901.98 & 282233.2 \\
\hline $23-067$ & FQ Tau AB & $04191281+2829330$ & $\mathrm{C} 04161+2822$ & HBC 377 , Haro 6-3 & 41912.81 & 282933.1 \\
\hline $24-058$ & FQ Tau AB & $04191281+2829330$ & $\mathrm{C} 04161+2822$ & HBC 377 , Haro 6-3 & 41912.81 & 282933.1 \\
\hline $28-100$ & BP Tau & $04191583+2906269$ & $04161+2859$ & HD 281934, HBC 32 & 41915.84 & 290626.9 \\
\hline 23-074 & V819 Tau AB & $04192625+2826142$ & $\mathrm{C} 04162+2819$ & HBC 378, TAP 27 & 41926.26 & 282614.3 \\
\hline 24-061 & V819 Tau AB & $04192625+2826142$ & $\mathrm{C} 04162+2819$ & HBC 378, TAP 27 & 41926.26 & 282614.3 \\
\hline $16-000$ & IRAS 04166+2706 & & $04166+2706$ & $\ldots$ & 41943.00 & $271333.7(\mathrm{~S})$ \\
\hline $16-000$ & IRAS 04169+2702 & $04195844+2709570$ & $04169+2702$ & $\ldots$ & 41958.45 & 270957.1 \\
\hline $11-000$ & CFHT-Tau 19 & $04210795+2702204$ & & $\ldots$ & 42107.95 & 270220.4 \\
\hline $11-000$ & IRAS $04181+2655$ & & $04181+2655$ & $\ldots$ & 42110.90 & $270206.0(\mathrm{~S})$ \\
\hline $11-000$ & IRAS $04181+2654 \mathrm{AB}$ & $04211146+2701094$ & $04181+2654$ & $\ldots$ & 42111.47 & 270109.4 \\
\hline $11-023$ & 2M J04213459 & $04213459+2701388$ & & $\ldots$ & 42134.60 & 270138.9 \\
\hline $01-028$ & IRAS 04187+1927 & $04214323+1934133$ & $04187+1927$ & $\ldots$ & 42143.24 & 193413.3 \\
\hline $11-037$ & CFHT-Tau 10 & $04214631+2659296$ & $\ldots$ & $\ldots$ & 42146.31 & 265929.6 \\
\hline $11-000$ & 2M J04215450+2652 & $04215450+2652315$ & & $\ldots$ & 42154.51 & 265231.5 \\
\hline $21-038$ & RY Tau & $04215740+2826355$ & $04188+2819$ & HBC 34, HD 283571 & 42157.40 & 282635.5 \\
\hline $21-039$ & HD 283572 & $04215884+2818066$ & $\mathrm{C} 04185+2811$ & V987 Tau, HBC 380 & 42158.84 & 281806.6 \\
\hline 01-045 & T Tau N(+Sab) & $04215943+1932063$ & $04190+1924$ & HD 284419, HBC 35 & 42159.43 & 193206.4 \\
\hline $11-054$ & Haro 6-5 B & $04220069+2657324$ & & Haro 381, FS Tau B & 42200.70 & 265732.5 \\
\hline $11-057$ & FS Tau AC & $04220217+2657304$ & $04189+2650$ & HBC 383, Haro 6-5 A & 42202.18 & 265730.5 \\
\hline $21-044$ & LkCa 21 & $04220313+2825389$ & C04185+2818 & V1071 Tau, HBC 382 & 42203.14 & 282539.0 \\
\hline 01-054 & RX J0422.1+1934 & $04220496+1934483$ & $\ldots$ & $\ldots$ & 42204.96 & 193448.3 \\
\hline $01-062$ & 2M J04221332+1934 & $04221332+1934392$ & & $\ldots$ & 42213.32 & 193439.2 \\
\hline $11-079$ & CFHT-Tau 21 & $04221675+2654570$ & $\ldots$ & $\ldots$ & 42216.76 & 265457.1 \\
\hline 02-013 & FV Tau AB & $04265352+2606543$ & $\mathrm{C} 04238+2600$ & HBC 386, Haro 6-8 & 42653.53 & 260654.4 \\
\hline $02-000$ & FV Tau/c AB & $04265440+2606510$ & $\ldots$ & HBC 387 & 42654.41 & 260651.0 \\
\hline 02-016 & KPNO-Tau 13 & $04265732+2606284$ & $\ldots$ & $\ldots$ & 42657.33 & 260628.4 \\
\hline 02-000 & DG Tau B & $04270266+2605304$ & $\ldots$ & $\ldots$ & 42702.66 & 260530.5 \\
\hline 02-022 & DG Tau A & $04270469+2606163$ & $04240+2559$ & HBC 37 & 42704.70 & 260616.3 \\
\hline 02-000 & KPNO-Tau 4 & $04272799+2612052$ & $\ldots$ & $\ldots$ & 42728.00 & 261205.3 \\
\hline $02-000$ & IRAS $04248+2612 \mathrm{AB}$ & $04275730+2619183$ & $04248+2612$ & HH 31 IRS 2 & 42757.31 & 261918.3 \\
\hline $15-020$ & JH 507 & $04292071+2633406$ & & $\mathrm{~J} 1-507$ & 42920.71 & 263340.7 \\
\hline 13-004 & GV Tau AB & $04292373+2433002$ & $04263+2426$ & HBC 389, Haro 6-10 AB & 42923.73 & 243300.3 \\
\hline $13-000$ & IRAS 04264+2433 & $04293008+2439550$ & $04264+2433$ & & 42930.08 & 243955.1 \\
\hline $15-040$ & DH Tau AB & $04294155+2632582$ & $04267+2626$ & HBC 38 & 42941.56 & 263258.3 \\
\hline $15-042$ & DI Tau AB & $04294247+2632493$ & $\ldots$ & HBC 39 & 42942.48 & 263249.3 \\
\hline $15-044$ & KPNO-Tau 5 & $04294568+2630468$ & $\ldots$ & $\ldots$ & 42945.68 & 263046.8 \\
\hline $14-006$ & IQ Tau A & $04295156+2606448$ & $04267+2600$ & HBC 41, LkHa 265 & 42951.56 & 260644.9 \\
\hline $13-000$ & CFHT-Tau 20 & $04295950+2433078$ & $\ldots$ & $\ldots$ & 42959.51 & 243307.9 \\
\hline $14-000$ & KPNO-Tau 6 & $04300724+2608207$ & $\ldots$ & $\ldots$ & 43007.24 & 260820.8 \\
\hline $13-035$ & FX Tau AB & $04302961+2426450$ & $04267+2420$ & HBC 44, Haro 6-11 & 43029.61 & 242645.0 \\
\hline $14-057$ & DK Tau AB & $04304425+2601244$ & $04276+2554$ & HBC 45 & 43044.25 & 260124.5 \\
\hline $14-000$ & KPNO-Tau 7 & $04305718+2556394$ & $\ldots$ & $\ldots$ & 43057.19 & 255639.5 \\
\hline $22-013$ & MHO 9 & $04311578+1820072$ & $\ldots$ & $\ldots$ & 43115.78 & 182007.2 \\
\hline $22-021$ & MHO 4 & $04312405+1800215$ & & RXJ0431.4+1800 & 43124.06 & 180021.5 \\
\hline $22-040$ & L1551 IRS5 & $04313407+1808049$ & $04287+1801$ & $\ldots$ & 43134.08 & 180804.9 \\
\hline $22-042$ & LkHa 358 & $04313613+1813432$ & & CoKu Tau 2, HBC 394 & 43136.13 & 181343.3 \\
\hline $22-000$ & HH 30 & $04313747+1812244$ & $04287+1806$ & V1213 Tau & 43137.47 & 181224.5 \\
\hline $22-043$ & HL Tau & $04313843+1813576$ & $04287+1807$ & HBC 49, Haro 6-14 & 43138.44 & 181357.7 \\
\hline $22-047$ & XZ Tau AB & $04314007+1813571$ & $\ldots$ & HBC 50, Haro 6-15 & 43140.07 & 181357.2 \\
\hline $22-056$ & L1551 NE & $04314444+1808315$ & $\ldots$ & $\ldots$ & 43144.45 & 180831.5 \\
\hline 03-005 & HK Tau AB & $04315056+2424180$ & $04288+2417$ & HBC 48, Haro 6-12 & 43150.57 & 242418.1 \\
\hline $22-070$ & V710 Tau BA & $04315779+1821380$ & $04290+1815$ & HBC $395+51$, LkHa266S & 43157.79 & 182138.1 \\
\hline 19-009 & JH 665 & $04315844+2543299$ & & $J 1-665$ & 43158.44 & 254329.9 \\
\hline 22-089 & L1551 51 & $04320926+1757227$ & $042916+1751$ & V1075 Tau, HBC 397 & 43209.27 & 175722.8 \\
\hline 22-097 & V827 Tau & $04321456+1820147$ & & НBC 399, ТAP 42 & 43214.57 & 182014.7 \\
\hline 03-016 & Haro 6-13 & $04321540+2428597$ & $04292+2422$ & V806 Tau, HBC 396 & 43215.41 & 242859.7 \\
\hline
\end{tabular}


Table 7. continued.

\begin{tabular}{|c|c|c|c|c|c|c|}
\hline XEST & Name & $2 \mathrm{MASS}^{a}$ & $\mathrm{IRAS}^{b}$ & Alternative names & $\begin{array}{l}\mathrm{RA}(\mathrm{J} 2000.0)^{c} \\
\mathrm{~h} \mathrm{~m} \mathrm{~s}\end{array}$ & $\begin{array}{l}\operatorname{Dec}(\mathrm{J} 2000.0)^{c} \\
\operatorname{deg} \prime^{\prime} \prime \prime\end{array}$ \\
\hline $22-100$ & V826 Tau & $04321583+1801387$ & $\ldots$ & HBC 400, TAP 43 & 43215.84 & 180138.7 \\
\hline 22-101 & MHO 5 & $04321606+1812464$ & $\ldots$ & $\ldots$ & 43216.07 & 181246.4 \\
\hline 03-017 & CFHT-Tau 7 & $04321786+2422149$ & $\ldots$ & $\ldots$ & 43217.86 & 242215.0 \\
\hline 03-019 & V928 Tau AB & $04321885+2422271$ & $\ldots$ & HK Tau/G2, JH 91, HBC 398 & 43218.86 & 242227.1 \\
\hline 03-022 & FY Tau & $04323058+2419572$ & $04294+2413$ & HBC 401, Haro 6-17 & 43230.58 & 241957.3 \\
\hline $03-023$ & FZ Tau & $04323176+2420029$ & $\ldots$ & HBC 402, Haro 6-18 & 43231.76 & 242003.0 \\
\hline $17-002$ & IRAS 04295+2251 & $04323205+2257266$ & $04295+2251$ & L1536 IRS & 43232.05 & 225726.7 \\
\hline 19-049 & UZ Tau E+W(AB) & $04324303+2552311$ & $04296+2546$ & HBC 52 & 43243.04 & 255231.1 \\
\hline 17-009 & JH 112 & $04324911+2253027$ & $04298+2246$ & $\ldots$ & 43249.11 & 225302.8 \\
\hline 03-031 & CFHT-Tau 5 & $04325026+2422115$ & $\ldots$ & $\ldots$ & 43250.27 & 242211.6 \\
\hline 04-003 & CFHT-Tau 5 & $04325026+2422115$ & $\ldots$ & $\ldots$ & 43250.27 & 242211.6 \\
\hline 03-035 & MHO 8 & $04330197+2421000$ & $\ldots$ & $\ldots$ & 43301.98 & 242100.0 \\
\hline 04-009 & MHO 8 & $04330197+2421000$ & & $\ldots$ & 43301.98 & 242100.0 \\
\hline 04-010 & GH Tau AB & $04330622+2409339$ & $04300+2403$ & HBC 55, Haro 6-20 & 43306.22 & 240934.0 \\
\hline 04-012 & V807 Tau SNab & $04330664+2409549$ & $\ldots$ & HBC 404, Elias 12 & 43306.64 & 240955.0 \\
\hline $18-004$ & KPNO-Tau 14 & $04330781+2616066$ & $\ldots$ & $\ldots$ & 43307.81 & 261606.6 \\
\hline $17-000$ & CFHT-Tau 12 & $04330945+2246487$ & $\ldots$ & $\ldots$ & 43309.46 & 224648.7 \\
\hline 04-016 & V830 Tau & $04331003+2433433$ & $\mathrm{C} 04301+2427$ & НBC 405, ТАР 46 & 43310.03 & 243343.4 \\
\hline $18-000$ & IRAS S04301+261 & $04331435+2614235$ & $\ldots$ & $\ldots$ & 43314.36 & 261423.5 \\
\hline $17-000$ & IRAS 04302+2247 & $04331650+2253204$ & $04302+2247$ & Butterfly Star & 43316.50 & 225320.4 \\
\hline $17-027$ & IRAS $04303+2240$ & $04331907+2246342$ & $04303+2240$ & L1536S & 43319.07 & 224634.2 \\
\hline 04-034 & GI Tau & $04333405+2421170$ & $04305+2414$ & HBC 56, Haro 6-21 & 43334.06 & 242117.0 \\
\hline 04-035 & GK Tau AB & $04333456+2421058$ & & HBC 57, Haro 6-22 & 43334.56 & 242105.9 \\
\hline 18-019 & IS Tau AB & $04333678+2609492$ & $04308+2607$ & HBC 59, Haro 6-23 & 43336.79 & 260949.2 \\
\hline $17-058$ & CI Tau & $04335200+2250301$ & $04308+2244$ & HBC 61, Haro 6-25 & 43352.00 & 225030.2 \\
\hline $18-030$ & IT Tau AB & $04335470+2613275$ & $\ldots$ & Haro 6-26 & 43354.70 & 261327.5 \\
\hline $17-066$ & JH 108 & $04341099+2251445$ & $\ldots$ & $\ldots$ & 43410.99 & 225144.5 \\
\hline $17-068$ & CFHT-BD Tau 1 & $04341527+2250309$ & $\ldots$ & $\ldots$ & 43415.27 & 225031.0 \\
\hline $25-026$ & AA Tau & $04345542+2428531$ & $04318+2422$ & НBC 63 & 43455.42 & 242853.2 \\
\hline 09-010 & HO Tau AB & $04352020+2232146$ & $\mathrm{C} 04323+2226$ & HBC 64, Haro 6-27 & 43520.20 & 223214.6 \\
\hline 08-019 & FF Tau AB & $04352089+2254242$ & $\ldots$ & НBC 409 & 43520.90 & 225424.2 \\
\hline $12-040$ & DN Tau & $04352737+2414589$ & $04324+2408$ & HBC 65 & 43527.37 & 241458.9 \\
\hline $12-000$ & IRAS $04325+2402 \mathrm{AB}$ & $04353539+2408194$ & $04325+2402$ & L1535 IRS & 43535.39 & 240819.4 \\
\hline $12-059$ & CoKu Tau 3 AB & $04354093+2411087$ & $\ldots$ & НBC 411 & 43540.94 & 241108.8 \\
\hline 09-022 & KPNO-Tau 8 & $04354183+2234115$ & $\ldots$ & $\ldots$ & 43541.84 & 223411.6 \\
\hline $08-037$ & HQ Tau AB & $04354733+2250216$ & $04327+2244$ & $\ldots$ & 43547.34 & 225021.7 \\
\hline 09-026 & HQ Tau AB & $04354733+2250216$ & $04327+2244$ & $\ldots$ & 43547.34 & 225021.7 \\
\hline 08-043 & KPNO-Tau 15 & $04355109+2252401$ & $\ldots$ & $\ldots$ & 43551.10 & 225240.1 \\
\hline 09-031 & KPNO-Tau 15 & $04355109+2252401$ & $\ldots$ & $\ldots$ & 43551.10 & 225240.1 \\
\hline $08-000$ & KPNO-Tau 9 & $04355143+2249119$ & $\ldots$ & $\ldots$ & 43551.43 & 224911.9 \\
\hline 09-000 & KPNO-Tau 9 & $04355143+2249119$ & $\ldots$ & $\ldots$ & 43551.43 & 224911.9 \\
\hline 08-048 & HP Tau AB & $04355277+2254231$ & $04328+2248$ & HBC 66, LkHa 258 & 43552.78 & 225423.1 \\
\hline 08-051a & HP Tau/G3 AB & $04355349+2254089$ & $\ldots$ & НBC 414 & 43553.50 & 225409.0 \\
\hline 08-051 & HP Tau/G2 & $04355415+2254134$ & $\ldots$ & V1025 Tau, HBC 415 & 43554.15 & 225413.5 \\
\hline 08-058 & Haro 6-28 AB & $04355684+2254360$ & $\ldots$ & V1026 Tau, HBC 416 & 43556.84 & 225436.0 \\
\hline 08-000 & CFHT-BD Tau 2 & $04361038+2259560$ & $\ldots$ & $\ldots$ & 43610.39 & 225956.0 \\
\hline 08-080 & CFHT-BD Tau 3 & $04363893+2258119$ & $\ldots$ & $\ldots$ & 43638.94 & 225811.9 \\
\hline $05-005$ & CFHT-Tau 6 & $04390396+2544264$ & $\ldots$ & $\ldots$ & 43903.96 & 254426.4 \\
\hline 05-000 & IRAS 04361+2547 & $04391389+2553208$ & $04361+2547$ & TMR 1, ITG 12 & 43913.89 & 255320.9 \\
\hline 05-013 & GN Tau AB & $04392090+2545021$ & $04362+2539$ & Haro 6-31 & 43920.91 & 254502.1 \\
\hline 05-017 & IRAS $04365+2535$ & $04393519+2541447$ & $04365+2535$ & TMC-1A & 43935.19 & 254144.7 \\
\hline 05-024 & IRAS 04369+2539 & $04395574+2545020$ & $04369+2539$ & Elias 18, IC 2087/IR & 43955.75 & 254502.0 \\
\hline 07-011 & JH 223 & $04404950+2551191$ & $\ldots$ & $\ldots$ & 44049.51 & 255119.2 \\
\hline $07-022$ & Haro 6-32 & $04410424+2557561$ & $\ldots$ & $\ldots$ & 44104.24 & 255756.1 \\
\hline 07-000 & ITG $33 \mathrm{~A}$ & $04410826+2556074$ & $\ldots$ & $\ldots$ & 44108.26 & 255607.5 \\
\hline 07-000 & CFHT-Tau 8 & $04411078+2555116$ & $\ldots$ & $\ldots$ & 44110.78 & 255511.7 \\
\hline 07-000 & IRAS 04381+2540 & $04411267+2546354$ & $04381+2540$ & TMC-1, ITG 35 & 44112.68 & 254635.4 \\
\hline 07-041 & IRAS $04385+2550 \mathrm{AB}$ & $04413882+2556267$ & $04385+2550$ & Haro 6-33 & 44138.82 & 255626.8 \\
\hline $10-017$ & CoKuLk332/G2 AB & $04420548+2522562$ & $\ldots$ & V999 Tau, HBC 422 & 44205.49 & 252256.3 \\
\hline
\end{tabular}


Table 7. continued.

\begin{tabular}{|c|c|c|c|c|c|c|}
\hline$\overline{\mathrm{XEST}}$ & Name & $2 \mathrm{MASS}^{a}$ & $\mathrm{IRAS}^{b}$ & Alternative names & $\begin{array}{l}\overline{\mathrm{RA}(\mathrm{J} 2000.0)^{c}} \\
\mathrm{~h} \mathrm{~m} \text { s }\end{array}$ & $\begin{array}{l}\operatorname{Dec}(\mathbf{J} 2000.0)^{c} \\
\operatorname{deg}, '{ }^{\prime}\end{array}$ \\
\hline $10-018$ & CoKuLk332/G1 AB & $04420732+2523032$ & & HBC 423 & 44207.33 & 252303.2 \\
\hline $10-020$ & V955 Tau AB & $04420777+2523118$ & $04390+2517$ & HBC 69, JH 226, LkHa 332 & 44207.77 & 252311.8 \\
\hline $10-034$ & CIDA 7 & $04422101+2520343$ & $\ldots$ & C31 & 44221.02 & 252034.4 \\
\hline $10-045$ & DP Tau & $04423769+2515374$ & $04395+2509$ & HBC 70 & 44237.70 & 251537.5 \\
\hline $10-060$ & GO Tau & $04430309+2520187$ & $\mathrm{C} 04400+2514$ & HBC 71 & 44303.09 & 252018.8 \\
\hline $26-012$ & $2 \mathrm{M} \mathrm{J} 04552333+30$ & $04552333+3027366$ & $\ldots$ & $\ldots$ & 45523.33 & 302736.6 \\
\hline $26-034$ & $2 \mathrm{M} \mathrm{J} 04554046+30$ & $04554046+3039057$ & $\ldots$ & $\ldots$ & 45540.46 & 303905.7 \\
\hline $26-043$ & AB Aur & $04554582+3033043$ & $04525+3028$ & HD 31293, HBC 78 & 45545.83 & 303304.4 \\
\hline $26-050$ & 2MJ04554757/801 & $04554757+3028077$ & & $\ldots$ & 45547.57 & 302807.7 \\
\hline $26-067$ & SU Aur & $04555938+3034015$ & $04528+3029$ & HBC 79, HD 282624 & 45559.38 & 303401.6 \\
\hline $26-072$ & НBC 427 & $04560201+3021037$ & $\ldots$ & V397 Aur & 45602.02 & 302103.8 \\
\hline \multicolumn{7}{|c|}{ Additional sources from Chandra } \\
\hline C1-0 & KPNO-Tau 10 & $04174955+2813318$ & & $\cdots$ & 41749.55 & 281331.9 \\
\hline C1-1 & IRAS $04158+2805$ & $04185813+2812234$ & $04158+2805$ & $\ldots$ & 41858.14 & 281223.5 \\
\hline $\mathrm{C} 2-1$ & Haro 6-5 B & $04220069+2657324$ & 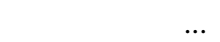 & Haro 381, FS Tau B & 42200.70 & 265732.5 \\
\hline $\mathrm{C} 2-2$ & FS Tau AC & $04220217+2657304$ & $04189+2650$ & HBC 383, Haro 6-5 A & 42202.18 & 265730.5 \\
\hline C3-1 & FV Tau/c AB & $04265440+2606510$ & $\ldots$ & HBC 387 & 42654.41 & 260651.0 \\
\hline C3-2 & DG Tau B & $04270266+2605304$ & & & 42702.66 & 260530.5 \\
\hline C4-1 & GV Tau AB & $04292373+2433002$ & $04263+2426$ & HBC 389, Haro 6-10 AB & 42923.73 & 243300.3 \\
\hline C5-2 & HN Tau AB & $04333935+1751523$ & & $\ldots$ & 43339.35 & 175152.4 \\
\hline C5-1 & L1551 55 & $04324373+1802563$ & $042950+1757$ & $\ldots$ & 43243.73 & 180256.3 \\
\hline C5-4 & HD 28867 & 04333297+1801004 & $04306+1754$ & HR 1442, L1551 53 & 43332.98 & 180100.4 \\
\hline C5-3 & DM Tau & 04334871+1810099 & $04309+1803$ & HBC 62 & 43348.72 & 181010.0 \\
\hline C6-1 & CFHT-BD Tau 4 & $04394748+2601407$ & $04368+2557 \mathrm{~N} 3$ & $\ldots$ & 43947.48 & 260140.8 \\
\hline C6-0 & L1527 IRS & $\ldots$ & $04368+2557$ & $\ldots$ & 43953.59 & $260305.5(\mathrm{~S})$ \\
\hline C6-0 & CFHT-Tau 17 & $04400174+2556292$ & & $\ldots$ & 44001.75 & 255629.2 \\
\hline C6-2 & IRAS $04370+2559$ & $04400800+2605253$ & $04370+2559$ & $\ldots$ & 44008.00 & 260525.4 \\
\hline
\end{tabular}

${ }^{a}$ Nearest 2MASS entry within $5^{\prime \prime}$ to coordinates given by references 5, 18, 33, or SIMBAD. Unlikely identifications in parentheses.

${ }^{b}$ Nearest IRAS catalog entry, within $10^{\prime \prime}$.

${ }^{c}$ 2MASS coordinates. For unlikely identifications, SIMBAD (S). 
M. Güdel et al.: XMM-Newton extended survey of Taurus, Online Material p 20

Table 8. Fundamental parameters of targets in XEST (2): Multiplicity.

\begin{tabular}{|c|c|c|c|c|}
\hline XEST & Name & Comp & $\begin{array}{r}\text { Separations } \\
\left({ }^{\prime \prime}\right)\end{array}$ & Refs \\
\hline $27-115$ & HBC 352 & 1 & 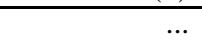 & ... \\
\hline $27-000$ & HBC 353 & 1 & 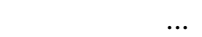 & \\
\hline $06-005$ & HBC 358 AB & 3 & $0.15,1.55$ & $11,20,31,40$ \\
\hline 06-007 & HBC 359 & 1 & $\ldots$ & $\ldots$ \\
\hline 06-059 & L1489 IRS & 1 & $\ldots$ & $\ldots$ \\
\hline $20-001$ & LkCa 1 & 1 & $\ldots$ & $\ldots$ \\
\hline $20-005$ & Anon 1 & 1 & $\ldots$ & $\ldots$ \\
\hline $20-000$ & IRAS $04108+2803 \mathrm{~A}$ & 1 & $\ldots$ & $\ldots$ \\
\hline $20-022$ & IRAS $04108+2803 \mathrm{~B}$ & 1 & $\ldots$ & $\ldots$ \\
\hline $20-000$ & 2M J04141188+28 & 1 & $\ldots$ & $\ldots$ \\
\hline $20-042$ & V773 Tau ABC & 4 & $0.002,0.11,0.2$ & $16,17,31,58,61,62$ \\
\hline $20-043$ & FM Tau & 1 & $\ldots$ & $\ldots$ \\
\hline $20-046$ & CW Tau & 1 & $\ldots$ & $\ldots$ \\
\hline $20-047$ & CIDA 1 & 1 & $\ldots$ & $\ldots$ \\
\hline $20-056$ & MHO 2/1 & 2 & 4.0 & 13 \\
\hline $20-058$ & MHO 3 & 1 & $\ldots$ & $\ldots$ \\
\hline $20-069$ & FO Tau AB & 2 & 0.16 & $16,20,31,58$ \\
\hline $20-073$ & CIDA 2 & 1 & $\ldots$ & $\ldots$ \\
\hline $23-002$ & CY Tau & 1 & $\ldots$ & $\ldots$ \\
\hline 24-002 & CY Tau & 1 & $\ldots$ & $\ldots$ \\
\hline 23-004 & LkCa 5 & 1 & $\ldots$ & $\ldots$ \\
\hline 24-004 & LkCa 5 & 1 & $\ldots$ & $\ldots$ \\
\hline $23-008$ & CIDA 3 & 1 & $\ldots$ & $\ldots$ \\
\hline $24-008$ & CIDA 3 & 1 & $\ldots$ & $\ldots$ \\
\hline $23-015$ & V410 X3 & 1 & $\ldots$ & $\ldots$ \\
\hline $24-015$ & V410 X3 & 1 & $\ldots$ & $\ldots$ \\
\hline $23-018$ & V410 A13 & 1 & $\ldots$ & $\ldots$ \\
\hline $24-000$ & V410 A13 & 1 & $\ldots$ & $\ldots$ \\
\hline $23-000$ & V410 A24 & 1 & $\ldots$ & $\ldots$ \\
\hline 24-000 & V410 A24 & 1 & $\ldots$ & $\ldots$ \\
\hline $23-029$ & V410 A25 & 1 & $\ldots$ & $\ldots$ \\
\hline $24-027$ & V410 A25 & 1 & $\ldots$ & $\ldots$ \\
\hline $23-032$ & V410 Tau ABC & 3 & $0.12,0.29$ & $16,17,58$ \\
\hline $24-028$ & V410 Tau ABC & 3 & $0.12,0.29$ & $16,17,58$ \\
\hline $23-033$ & DD Tau AB & 2 & 0.54 & $16,20,31,40,47,58$ \\
\hline $24-029$ & DD Tau AB & 2 & 0.54 & $16,20,31,40,47,58$ \\
\hline $23-035$ & CZ Tau AB & 2 & 0.33 & $31,40,58$ \\
\hline $24-030$ & CZ Tau AB & 2 & 0.33 & $31,40,58$ \\
\hline $23-036$ & IRAS $04154+2823$ & 1 & $\ldots$ & $\ldots$ \\
\hline $24-031$ & IRAS 04154+2823 & 1 & $\ldots$ & $\ldots$ \\
\hline $23-037$ & V410 X2 & 1 & $\ldots$ & $\ldots$ \\
\hline $24-032$ & V410 X2 & 1 & $\ldots$ & $\ldots$ \\
\hline $23-045$ & V410 X4 & 1 & $\ldots$ & $\ldots$ \\
\hline $24-038$ & V410 X4 & 1 & $\ldots$ & $\ldots$ \\
\hline $23-047$ & V892 Tau & 3 & $0.05,4$ & 32,54 \\
\hline $24-040$ & V892 Tau & 3 & $0.05,4$ & 32,54 \\
\hline $23-048$ & LR 1 & 1 & $\ldots$ & $\ldots$ \\
\hline $24-000$ & LR 1 & 1 & $\ldots$ & $\ldots$ \\
\hline $23-050$ & V410 X7 & 1 & $\ldots$ & $\ldots$ \\
\hline 24-042 & V410 X7 & 1 & $\ldots$ & $\ldots$ \\
\hline $23-000$ & V410 A20 & 1 & $\ldots$ & $\ldots$ \\
\hline $24-000$ & V410 A20 & 1 & $\ldots$ & $\ldots$ \\
\hline $23-056$ & Hubble 4 & 1 & $\ldots$ & $\ldots$ \\
\hline $24-047$ & Hubble 4 & 1 & $\ldots$ & $\ldots$ \\
\hline $23-000$ & KPNO-Tau 2 & 1 & $\ldots$ & $\ldots$ \\
\hline $24-000$ & KPNO-Tau 2 & 1 & $\ldots$ & $\ldots$ \\
\hline $23-000$ & CoKu Tau 1 & 1 & $\ldots$ & $\ldots$ \\
\hline $24-000$ & CoKu Tau 1 & 1 & $\ldots$ & $\ldots$ \\
\hline $23-061$ & V410 X6 & 1 & $\ldots$ & $\ldots$ \\
\hline
\end{tabular}


Table 8. continued.

\begin{tabular}{|c|c|c|c|c|}
\hline XEST & Name & Comp & $\begin{array}{r}\text { Separations } \\
\left({ }^{\prime \prime}\right)\end{array}$ & Refs \\
\hline $24-054$ & V410 X6 & 1 & $\ldots$ & $\ldots$ \\
\hline $23-063$ & V410 X5 & 1 & $\ldots$ & $\ldots$ \\
\hline $24-055$ & V410 X5 & 1 & $\ldots$ & $\ldots$ \\
\hline $23-067$ & FQ Tau AB & 2 & 0.73 & $20,31,40,58$ \\
\hline $24-058$ & FQ Tau AB & 2 & 0.73 & $20,31,40,58$ \\
\hline $28-100$ & BP Tau & 1 & & $\ldots$ \\
\hline 23-074 & V819 Tau AB & 2 & 10.5 & $31^{a}$ \\
\hline $24-061$ & V819 Tau AB & 2 & 10.5 & $31^{a}$ \\
\hline $16-000$ & IRAS 04166+2706 & 1 & $\ldots$ & $\ldots$ \\
\hline $16-000$ & IRAS 04169+2702 & 1 & $\ldots$ & $\ldots$ \\
\hline $11-000$ & CFHT-Tau 19 & 1 & $\ldots$ & $\ldots$ \\
\hline $11-000$ & IRAS $04181+2655$ & 1 & $\ldots$ & $\ldots$ \\
\hline $11-000$ & IRAS $04181+2654 \mathrm{AB}$ & 2 & $\ldots$ & ... \\
\hline $11-023$ & 2M J04213459 & 1 & $\ldots$ & ... \\
\hline $01-028$ & IRAS 04187+1927 & 1 & $\ldots$ & $\ldots$ \\
\hline $11-037$ & CFHT-Tau 10 & 1 & $\ldots$ & $\ldots$ \\
\hline $11-000$ & $2 \mathrm{M} \mathrm{J} 04215450+2652$ & 1 & $\ldots$ & ... \\
\hline $21-038$ & RY Tau & 1 & $\ldots$ & $\ldots$ \\
\hline 21-039 & HD 283572 & 1 & $\ldots$ & \\
\hline 01-045 & T Tau N(+Sab) & 3 & $0.67,0.13$ & $12,47,58$ \\
\hline $11-054$ & Haro 6-5 B & 1 & $\ldots$ & $\ldots$ \\
\hline $11-057$ & FS Tau AC & 2 & 0.30 & $20,31,40,52,53,58$ \\
\hline 21-044 & LkCa 21 & 1 & $\ldots$ & $\ldots$ \\
\hline $01-054$ & RX J0422.1+1934 & 1 & $\ldots$ & $\ldots$ \\
\hline $01-062$ & 2M J04221332+1934 & 1 & $\ldots$ & $\ldots$ \\
\hline $11-079$ & CFHT-Tau 21 & 1 & $\ldots$ & $\ldots$ \\
\hline 02-013 & FV Tau AB & 2 & 0.71 & $16,31,40,47,53,58$ \\
\hline 02-000 & FV Tau/c AB & 2 & 0.67 & $31,40,52,53,58$ \\
\hline 02-016 & KPNO-Tau 13 & 1 & $\ldots$ & $\ldots$ \\
\hline 02-000 & DG Tau B & 1 & $\cdots$ & ... \\
\hline $02-022$ & DG Tau A & 1 & $\ldots$ & $\ldots$ \\
\hline $02-000$ & KPNO-Tau 4 & 1 & $\ldots$ & $\ldots$ \\
\hline $02-000$ & IRAS $04248+2612 \mathrm{AB}$ & 2 & 4.55 & 13 \\
\hline $15-020$ & JH 507 & 1 & $\ldots$ & $\ldots$ \\
\hline 13-004 & GV Tau AB & 2 & 1.2 & $30,31,47,53,58$ \\
\hline $13-000$ & IRAS $04264+2433$ & 1 & $\ldots$ & $\ldots$ \\
\hline $15-040$ & DH Tau AB & 2 & 2.3 & 25 \\
\hline $15-042$ & DI Tau AB & 2 & 0.12 & 16,53 \\
\hline $15-044$ & KPNO-Tau 5 & 1 & $\ldots$ & $\ldots$ \\
\hline $14-006$ & IQ Tau A & 1 & $\ldots$ & $b$ \\
\hline $13-000$ & CFHT-Tau 20 & 1 & $\cdots$ & ... \\
\hline $14-000$ & KPNO-Tau 6 & 1 & $\ldots$ & \\
\hline $13-035$ & FX Tau AB & 2 & 0.85 & $11,16,31,40,53,58$ \\
\hline $14-057$ & DK Tau AB & 2 & 2.27 & $11,31,40,43,47,52,53,58$ \\
\hline $14-000$ & KPNO-Tau 7 & 1 & $\ldots$ & $\ldots$ \\
\hline $22-013$ & MHO 9 & 1 & $\cdots$ & ... \\
\hline $22-021$ & MHO 4 & 1 & $\ldots$ & $\ldots$ \\
\hline $22-040$ & L1551 IRS5 & 2 & 0.3 & 51 \\
\hline $22-042$ & LkHa 358 & 1 & $\ldots$ & $\ldots$ \\
\hline $22-000$ & HH 30 & 1 & $\ldots$ & ... \\
\hline 22-043 & HL Tau & 1 & $\ldots$ & $\ldots$ \\
\hline 22-047 & XZ Tau AB & 2 & 0.3 & $16,20,31,47,58$ \\
\hline $22-056$ & L1551 NE & 2 & 0.5 & 64 \\
\hline 03-005 & HK Tau AB & 2 & 2.4 & $11,31,40,42,43,53,58$ \\
\hline $22-070$ & V710 Tau BA & 2 & 3.1 & $21,31,40,43,58$ \\
\hline 19-009 & JH 665 & 1 & $\cdots$ & $\ldots$ \\
\hline 22-089 & L1551 51 & 1 & $\ldots$ & $\ldots$ \\
\hline $22-097$ & V827 Tau & 1 & $\cdots$ & ... \\
\hline 03-016 & Haro 6-13 & 1 & $\ldots$ & $\ldots$ \\
\hline
\end{tabular}


Table 8. continued.

\begin{tabular}{|c|c|c|c|c|}
\hline XEST & Name & Comp & $\begin{array}{r}\text { Separations } \\
\left({ }^{\prime \prime}\right)\end{array}$ & Refs \\
\hline $22-100$ & V826 Tau & 1 & $\ldots$ & $\ldots$ \\
\hline 22-101 & MHO 5 & 1 & $\ldots$ & $\ldots$ \\
\hline 03-017 & CFHT-Tau 7 & 1 & $\ldots$ & $\ldots$ \\
\hline 03-019 & V928 Tau AB & 2 & 0.17 & $16,31,53,58$ \\
\hline 03-022 & FY Tau & 1 & $\ldots$ & $c$ \\
\hline $03-023$ & FZ Tau & 1 & $\ldots$ & $\ldots$ \\
\hline $17-002$ & IRAS 04295+2251 & 1 & $\ldots$ & $\ldots$ \\
\hline 19-049 & UZ Tau E+W(AB) & 3 & $3.5,0.36$ & $16,17,20,21,31,40,52,53,58$ \\
\hline 17-009 & JH 112 & 1 & $\ldots$ & ... \\
\hline 03-031 & CFHT-Tau 5 & 1 & $\ldots$ & $\ldots$ \\
\hline 04-003 & CFHT-Tau 5 & 1 & $\ldots$ & $\ldots$ \\
\hline 03-035 & MHO 8 & 1 & $\ldots$ & $\ldots$ \\
\hline 04-009 & MHO 8 & 1 & $\ldots$ & $\ldots$ \\
\hline 04-010 & GH Tau AB & 2 & 0.31 & $16,31,40,58$ \\
\hline 04-012 & V807 Tau SNab & 3 & $0.3,0.023$ & $16,20,31,53,58$ \\
\hline $18-004$ & KPNO-Tau 14 & 1 & $\ldots$ & $\ldots$ \\
\hline $17-000$ & CFHT-Tau 12 & 1 & $\ldots$ & $\ldots$ \\
\hline 04-016 & V830 Tau & 1 & $\ldots$ & $\ldots$ \\
\hline $18-000$ & IRAS S04301+261 & 1 & $\ldots$ & $\ldots$ \\
\hline $17-000$ & IRAS 04302+2247 & 1 & $\ldots$ & $\ldots$ \\
\hline $17-027$ & IRAS $04303+2240$ & 1 & $\ldots$ & $\ldots$ \\
\hline 04-034 & GI Tau & 1 & $\ldots$ & $\ldots$ \\
\hline 04-035 & GK Tau AB & 2 & 2.5 & 21,49 \\
\hline $18-019$ & IS Tau AB & 2 & 0.22 & $16,20,53,58$ \\
\hline $17-058$ & CI Tau & 1 & & $\ldots$ \\
\hline $18-030$ & IT Tau AB & 2 & 2.4 & $11,40,52,53,58$ \\
\hline $17-066$ & JH 108 & 1 & $\ldots$ & $\ldots$ \\
\hline 17-068 & CFHT-BD Tau 1 & 1 & $\ldots$ & $\ldots$ \\
\hline $25-026$ & AA Tau & 1 & $\ldots$ & $\ldots$ \\
\hline 09-010 & HO Tau AB & 2 & 6.9 & $21^{d}$ \\
\hline 08-019 & FF Tau AB & 2 & 0.03 & 52,53 \\
\hline $12-040$ & DN Tau & 1 & $\ldots$ & $\ldots$ \\
\hline $12-000$ & IRAS $04325+2402 \mathrm{AB}$ & 1 & 8.15 & 13 \\
\hline $12-059$ & CoKu Tau 3 AB & 2 & 2.06 & $31,53,58$ \\
\hline 09-022 & KPNO-Tau 8 & 1 & $\ldots$ & $\ldots$ \\
\hline 08-037 & HQ Tau AB & 2 & 0.009 & $52^{e}$ \\
\hline 09-026 & HQ Tau AB & 2 & 0.009 & $52^{e}$ \\
\hline $08-043$ & KPNO-Tau 15 & 1 & $\ldots$ & $\ldots$ \\
\hline 09-031 & KPNO-Tau 15 & 1 & $\ldots$ & $\ldots$ \\
\hline 08-000 & KPNO-Tau 9 & 1 & $\ldots$ & $\ldots$ \\
\hline 09-000 & KPNO-Tau 9 & 1 & $\ldots$ & $\ldots$ \\
\hline 08-048 & HP Tau AB & 2 & 0.017 & 50,53 \\
\hline $08-051 \mathrm{a}$ & HP Tau/G3 AB & 2 & 0.022 & 50,53 \\
\hline $08-051$ & HP Tau/G2 & 1 & & $\ldots$ \\
\hline 08-058 & Haro 6-28 AB & 2 & 0.65 & $20,31,40,53,58$ \\
\hline 08-000 & CFHT-BD Tau 2 & 1 & $\ldots$ & $\ldots$ \\
\hline 08-080 & CFHT-BD Tau 3 & 1 & $\ldots$ & $\ldots$ \\
\hline 05-005 & CFHT-Tau 6 & 1 & $\ldots$ & $\ldots$ \\
\hline $05-000$ & IRAS 04361+2547 & 1 & $\ldots$ & $\ldots$ \\
\hline 05-013 & GN Tau AB & 2 & 0.36 & $52,53,58,67^{f}$ \\
\hline 05-017 & IRAS $04365+2535$ & 1 & $\ldots$ & $\ldots$ \\
\hline $05-024$ & IRAS 04369+2539 & 1 & ... & $\ldots$ \\
\hline 07-011 & JH 223 & 1 & ... & $\ldots$ \\
\hline $07-022$ & Haro 6-32 & 1 & $\ldots$ & $\ldots$ \\
\hline $07-000$ & ITG 33 A & 1 & $\ldots$ & $g$ \\
\hline 07-000 & CFHT-Tau 8 & 1 & $\ldots$ & $\ldots$ \\
\hline $07-000$ & IRAS 04381+2540 & 1 & $\ldots$ & $\ldots$ \\
\hline $07-041$ & IRAS $04385+2550 \mathrm{AB}$ & 2 & 18.9 & 13 \\
\hline $10-017$ & CoKuLk332/G2 AB & 2 & 0.27 & $20,31,58$ \\
\hline
\end{tabular}


Table 8. continued.

\begin{tabular}{llrrr}
\hline \hline XEST & Name & Comp & $\begin{array}{r}\text { Separations } \\
\left({ }^{\prime \prime}\right.\end{array}$ & Refs \\
\hline $10-018$ & CoKuLk332/G1 AB & 2 & 0.23 & $16,31,58$ \\
$10-020$ & V955 Tau AB & 2 & 0.34 & $20,31,40,58$ \\
$10-034$ & CIDA 7 & 1 & $\ldots$ & $\ldots$ \\
$10-045$ & DP Tau & 1 & $\ldots$ & $\ldots$ \\
$10-060$ & GO Tau & 1 & $\ldots$ & $\ldots$ \\
$26-012$ & 2M J04552333+30 & 1 & $\ldots$ & $\ldots$ \\
$26-034$ & 2M J04554046+30 & 1 & $\ldots$ & $\ldots$ \\
$26-043$ & AB Aur & 1 & $\ldots$ & $\ldots$ \\
$26-050$ & 2MJ04554757/801 & 1 & $\ldots$ & $\ldots$ \\
$26-067$ & SU Aur & 1 & $\ldots$ & $\ldots$ \\
$26-072$ & HBC 427 & 1 & $\ldots$ & $\ldots$ \\
\hline Additional sources from Chandra & & & $\ldots$ \\
\hline C1-0 & KPNO-Tau 10 & 1 & $\ldots$ & $\ldots$ \\
C1-1 & IRAS 04158+2805 & 1 & $\ldots$ & $\ldots$ \\
C2-1 & Haro 6-5 B & 1 & $\ldots$ & $\ldots$ \\
C2-2 & FS Tau AC & 2 & 0.25 & $20,31,52,53,58$ \\
C3-1 & FV Tau/c AB & 2 & 0.67 & $31,40,52,53,58$ \\
C3-2 & DG Tau B & 1 & $\ldots$ & $\ldots$ \\
C4-1 & GV Tau AB & 2 & 1.2 & $30,31,47,53,58$ \\
C5-2 & HN Tau AB & 2 & 3.1 & $11,21,31,42,43,58$ \\
C5-1 & L1551 55 & 1 & $\ldots$ & $\ldots$ \\
C5-4 & HD 28867 & 3 & 3.08 & $\ldots$ \\
C5-3 & DM Tau & 1 & $\ldots$ & $\ldots$ \\
C6-1 & CFHT-BD Tau 4 & 1 & $\ldots$ & $\ldots$ \\
C6-0 & L1527 IRS & 1 & $\ldots$ & $\ldots$ \\
C6-0 & CFHT-Tau 17 & 1 & $\ldots$ & $\ldots$ \\
C6-2 & IRAS 04370+2559 & 1 & $\ldots$ & \\
\hline & & & & $\ldots$ \\
\hline
\end{tabular}

${ }^{a}$ V819 Tau B suspected by Woitas (2001) to be a background star.

${ }^{b}$ IQ Tau B, 10.2" from IQ Tau A = XEST-14-006, has been interpreted as an extincted background $\mathrm{G}$ star by Hartigan et al. (1994).

${ }^{c}$ FY Tau is a suspected binary with separation of $0.153^{\prime \prime}$ (Richichi et al. 1994).

${ }^{d}$ HO Tau B suspected by Hartigan et al. (1994) to be a background star.

${ }^{e}$ HQ Tau B not confirmed by Simon et al. (1995).

${ }^{f}$ GN Tau AB: A component detected from lunar occulation by Simon et al. (1995) at a projected separation of $0.041^{\prime \prime}$ may be identical to GN Tau B at $\approx 0.36^{\prime \prime}$ (Simon et al. 1996)

${ }^{g}$ ITG 33 B, 5.2" from ITG 33 A, has been interpreted as a reddened background object by Martín (2000). 
Table 9. Fundamental parameters of targets in XEST (3): Photometry and spectroscopy.

\begin{tabular}{|c|c|c|c|c|c|c|c|c|}
\hline XEST & Name & $\mathrm{Spec}^{a}$ & Refs. & $\begin{array}{r}A_{\mathrm{V}}^{a} \\
(\mathrm{mag})\end{array}$ & $\begin{array}{r}A_{J} \\
(\mathrm{mag})\end{array}$ & $\begin{array}{l}T_{\text {eff }}^{a} \\
(\mathrm{~K})\end{array}$ & $\begin{array}{r}L_{*}^{b} \\
\left(L_{\odot}\right)\end{array}$ & Refs \\
\hline $27-115$ & HBC 352 & G0 & 27 & 0.87 & 0.25 & 6030 & 0.740 & 27 \\
\hline $27-000$ & HBC 353 & G5 & 27 & 0.97 & 0.28 & 5770 & 0.500 & 27 \\
\hline $06-005$ & HBC 358 AB & M2 & 27 & 0.21 & 0.06 & 3580 & 0.280 & 27 \\
\hline 06-007 & HBC 359 & M2 & 27 & 0.49 & 0.14 & 3580 & 0.260 & 27 \\
\hline $06-059$ & L1489 IRS & K4 & 59 & 10.20 & $\ldots$ & 4500 & 4.900 & 10,59 \\
\hline $20-001$ & LkCa 1 & M4 & 5 & 0.00 & 0.00 & 3270 & 0.380 & 5,27 \\
\hline $20-005$ & Anon 1 & M0 & 5 & 1.32 & 1.03 & 3850 & 2.600 & 5,27 \\
\hline $20-000$ & IRAS $04108+2803$ A & $\ldots$ & $\ldots$ & $\ldots$ & $\ldots$ & $\ldots$ & $\ldots$ & $\ldots$ \\
\hline $20-022$ & IRAS $04108+2803$ B & $\ldots$ & $\ldots$ & $\ldots$ & $\ldots$ & 3500 & 0.400 & 10 \\
\hline $20-000$ & 2M J04141188+28 & M6.25 & 33 & $\ldots$ & 0.28 & 2962 & 0.015 & 33 \\
\hline $20-042$ & V773 Tau ABC & $\mathrm{K} 2 / \mathrm{M} 0$ & 58 & 1.39 & 0.31 & $4898 / 3873$ & $1.89 / 1.17 / 5.60$ & 5,58 \\
\hline $20-043$ & FM Tau & M0 & 5 & 0.69 & 0.59 & 3850 & 0.460 & 5,27 \\
\hline $20-046$ & CW Tau & K3 & 5 & 2.29 & 0.55 & 4730 & 1.100 & 5,27 \\
\hline $20-047$ & CIDA 1 & M5.5 & 57 & 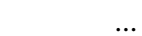 & 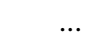 & 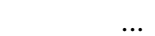 & 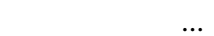 & , \\
\hline $20-056$ & MHO 2/1 & M2.5/2.5 & 5 & 4.58 & 1.85 & $3488 / 3488$ & $0.42 / 0.14 / 0.56$ & 5,4 \\
\hline $20-058$ & MHO 3 & K7 & 5 & 6.01 & 2.15 & 4060 & 0.910 & 5,4 \\
\hline $20-069$ & FO Tau AB & M2 & 5 & 1.87 & 0.63 & $3556 / 3556$ & $0.48 / 0.45 / 0.77$ & $5,58,27$ \\
\hline $20-073$ & CIDA 2 & M5.5 & 5 & 0.83 & 0.28 & 3058 & 0.320 & 5,27 \\
\hline $23-002$ & CY Tau & M1.5 & 5 & 0.10 & 0.23 & 3632 & 0.500 & 5,27 \\
\hline $24-002$ & CY Tau & M1.5 & 5 & 0.10 & 0.23 & 3632 & 0.500 & 5,27 \\
\hline 23-004 & LkCa 5 & M2 & 27 & 0.10 & 0.11 & 3560 & 0.370 & 5,27 \\
\hline 24-004 & LkCa 5 & M2 & 27 & 0.10 & 0.11 & 3560 & 0.370 & 5,27 \\
\hline $23-008$ & CIDA 3 & M4 & 5 & $\ldots$ & 0.26 & 3270 & 0.140 & 5 \\
\hline $24-008$ & CIDA 3 & M4 & 5 & $\ldots$ & 0.26 & 3270 & 0.140 & 5 \\
\hline 23-015 & V410 X3 & M6 & 5 & 0.54 & 0.17 & 2990 & 0.084 & 5,58 \\
\hline $24-015$ & V410 X3 & M6 & 5 & 0.54 & 0.17 & 2990 & 0.084 & 5,58 \\
\hline $23-018$ & V410 A13 & M5.75 & 5 & 2.78 & 0.79 & 3024 & 0.039 & 5,4 \\
\hline $24-000$ & V410 A13 & M5.75 & 5 & 2.78 & 0.79 & 3024 & 0.039 & 5,4 \\
\hline $23-000$ & V410 A24 & G1 & 5 & $\ldots$ & 6.73 & 5945 & 2.800 & 5 \\
\hline $24-000$ & V410 A24 & G1 & 5 & $\ldots$ & 6.73 & 5945 & 2.800 & 5 \\
\hline $23-029$ & V410 A25 & M1 & 5 & $\ldots$ & 6.55 & 3705 & 1.600 & 5 \\
\hline $24-027$ & V410 A25 & M1 & 5 & $\ldots$ & 6.55 & 3705 & 1.600 & 5 \\
\hline $23-032$ & V410 Tau ABC & K4 & 58 & 0.67 & 0.00 & $4602 / 3076$ & $2.15 / 0.05 / 2.20$ & 58 \\
\hline $24-028$ & V410 Tau ABC & K4 & 58 & 0.67 & 0.00 & $4602 / 3076$ & $2.15 / 0.05 / 2.20$ & 58 \\
\hline $23-033$ & DD Tau AB & M3 & 5 & $0.39 / 0.39$ & 0.05 & $3412 / 3412$ & $0.13 / 0.13 / 0.34$ & 5,58 \\
\hline $24-029$ & DD Tau AB & M3 & 5 & $0.39 / 0.39$ & 0.05 & $3412 / 3412$ & $0.13 / 0.13 / 0.34$ & 5,58 \\
\hline $23-035$ & CZ Tau AB & M3 & 5 & 1.32 & 0.48 & 3415 & 0.270 & 5,27 \\
\hline $24-030$ & CZ Tau AB & M3 & 5 & 1.32 & 0.48 & 3415 & 0.270 & 5,27 \\
\hline $23-036$ & IRAS $04154+2823$ & M2.5 & 5 & $\ldots$ & 4.26 & 3488 & 0.130 & 5 \\
\hline 24-031 & IRAS $04154+2823$ & M2.5 & 5 & $\ldots$ & 4.26 & 3488 & 0.130 & 5 \\
\hline $23-037$ & V410 X2 & M0 & 5 & $\ldots$ & 6.03 & 3850 & 3.000 & 5 \\
\hline $24-032$ & V410 X2 & M0 & 5 & $\ldots$ & 6.03 & 3850 & 3.000 & 5 \\
\hline 23-045 & V410 X4 & M4 & 5 & $\ldots$ & 5.29 & 3270 & 1.300 & 5 \\
\hline $24-038$ & V410 X4 & M4 & 5 & $\ldots$ & 5.29 & 3270 & 1.300 & 5 \\
\hline $23-047$ & V892 Tau & B9 & 5 & 5.93 & 2.39 & 10500 & 77.000 & 5,27 \\
\hline $24-040$ & V892 Tau & B9 & 5 & 5.93 & 2.39 & 10500 & 77.000 & 5,27 \\
\hline $23-048$ & LR 1 & K4.5 & 5 & $\ldots$ & 6.40 & 4470 & 0.470 & 5 \\
\hline $24-000$ & LR 1 & K4.5 & 5 & $\ldots$ & 6.40 & 4470 & 0.470 & 5 \\
\hline $23-050$ & V410 X7 & M0.75 & 5 & 8.18 & 2.34 & 3741 & 0.500 & 5,58 \\
\hline $24-042$ & V410 X7 & M0.75 & 5 & 8.18 & 2.34 & 3741 & 0.500 & 5,58 \\
\hline $23-000$ & V410 A20 & K3 & 5 & $\ldots$ & 6.57 & 4730 & 0.510 & 5 \\
\hline $24-000$ & V410 A20 & K3 & 5 & $\ldots$ & 6.57 & 4730 & 0.510 & 5 \\
\hline $23-056$ & Hubble 4 & K7 & 5 & 0.76 & 0.68 & 4060 & 2.700 & 5,27 \\
\hline $24-047$ & Hubble 4 & K7 & 5 & 0.76 & 0.68 & 4060 & 2.700 & 5,27 \\
\hline $23-000$ & KPNO-Tau 2 & M6.75 & 18 & 0.37 & 0.00 & 2889 & 0.007 & 18,5 \\
\hline $24-000$ & KPNO-Tau 2 & M6.75 & 18 & 0.37 & 0.00 & 2889 & 0.007 & 18,5 \\
\hline $23-000$ & CoKu Tau 1 & K7 & 59 & 6.80 & $\ldots$ & 4000 & 0.150 & 59 \\
\hline $24-000$ & CoKu Tau 1 & K7 & 59 & 6.80 & $\ldots$ & 4000 & 0.150 & 59 \\
\hline $23-061$ & V410 X6 & M5.5 & 5 & $\ldots$ & 0.17 & 3058 & 0.200 & 5 \\
\hline
\end{tabular}


Table 9. continued.

\begin{tabular}{|c|c|c|c|c|c|c|c|c|}
\hline XEST & Name & $\operatorname{Spec}^{a}$ & Refs. & $\begin{array}{r}A_{\mathrm{V}}^{a} \\
(\mathrm{mag}) \\
\end{array}$ & $\begin{array}{r}A_{J} \\
(\mathrm{mag})\end{array}$ & $\begin{array}{l}T_{\text {eff }}^{a} \\
(\mathrm{~K}) \\
\end{array}$ & $\begin{array}{r}L_{*}^{b} \\
\left(L_{\odot}\right) \\
\end{array}$ & Refs. \\
\hline $24-054$ & V410 X6 & M5.5 & 5 & & 0.17 & 3058 & 0.200 & 5 \\
\hline $23-063$ & V410 X5 & M5.5 & 5 & 3.36 & 0.72 & 3058 & 0.083 & 5,58 \\
\hline $24-055$ & V410 X5 & M5.5 & 5 & 3.36 & 0.72 & 3058 & 0.083 & 5,58 \\
\hline $23-067$ & FQ Tau AB & M3/M3.5 & 20 & $1.95 / 1.80$ & 0.16 & $3416 / 3345$ & $0.20 / 0.28 / 0.21$ & 5,20 \\
\hline $24-058$ & FQ Tau AB & M3/M3.5 & 20 & $1.95 / 1.80$ & 0.16 & $3416 / 3345$ & $0.20 / 0.28 / 0.21$ & 5,20 \\
\hline $28-100$ & BP Tau & K7 & 27 & 0.49 & 0.14 & 4060 & 0.950 & 27 \\
\hline $23-074$ & V819 Tau AB & K7 & 5 & 1.35 & 0.48 & 4060 & 0.910 & 5,27 \\
\hline 24-061 & V819 Tau AB & K7 & 5 & 1.35 & 0.48 & 4060 & 0.910 & 5,27 \\
\hline $16-000$ & IRAS 04166+2706 & $<\mathrm{M} 0$ & 66 & $\ldots$ & $\ldots$ & $\ldots$ & & $\ldots$ \\
\hline $16-000$ & IRAS $04169+2702$ & $\ldots$ & $\ldots$ & $\ldots$ & $\ldots$ & $\ldots$ & 0.800 & 13 \\
\hline $11-000$ & CFHT-Tau 19 & M5.25 & 18 & 7.30 & $\ldots$ & 3100 & 0.072 & 18 \\
\hline $11-000$ & IRAS 04181+2655 & $\ldots$ & $\ldots$ & $\ldots$ & $\ldots$ & 4000 & 1.800 & 10 \\
\hline $11-000$ & IRAS $04181+2654 \mathrm{AB}$ & & $\ldots$ & $\ldots$ & $\ldots$ & & $0.25 / 0.25 / 0.50$ & 13 \\
\hline $11-023$ & 2M J04213459 & M5.5 & 33 & $\ldots$ & 0.49 & 3058 & 0.065 & 33 \\
\hline $01-028$ & IRAS 04187+1927 & M0 & 27 & $\ldots$ & $\ldots$ & 3850 & & 27 \\
\hline $11-037$ & CFHT-Tau 10 & M5.75 & 66 & 3.59 & $\ldots$ & 3030 & 0.021 & 18,19 \\
\hline $11-000$ & 2M J04215450+2652 & M8.5 & 66 & 2.97 & $\ldots$ & 2642 & 0.003 & 19 \\
\hline $21-038$ & RY Tau & K1 & 27 & 1.84 & 0.53 & 5080 & 7.600 & 27 \\
\hline $21-039$ & HD 283572 & G5 & 27 & 0.38 & 0.11 & 5770 & 6.500 & 27 \\
\hline $01-045$ & T Tau N(+Sab) & K0 & 27 & 1.39 & 0.40 & 5250 & 8.910 & 27 \\
\hline $11-054$ & Haro 6-5 B & K5 & 59 & 9.96 & ... & 4395 & 0.047 & 59 \\
\hline $11-057$ & FS Tau AC & M0/M3.5 & 20 & $4.95 / 5.15$ & 0.53 & $3876 / 3345$ & $0.15 / 0.17 / 0.32$ & 27,20 \\
\hline 21-044 & LkCa 21 & M3 & 27 & 0.73 & 0.21 & 3470 & 0.620 & 27 \\
\hline 01-054 & RX J0422.1+1934 & $\ldots$ & $\ldots$ & $\ldots$ & $\ldots$ & $\ldots$ & $\cdots$ & $\ldots$ \\
\hline $01-062$ & 2M J04221332+1934 & M8 & 66 & 1.02 & $\ldots$ & 2713 & 0.017 & 19 \\
\hline $11-079$ & CFHT-Tau 21 & M1.25 & 18 & 6.60 & $\ldots$ & 3665 & 0.381 & 18 \\
\hline $02-013$ & FV Tau AB & K5/K6 & 58 & $5.33 / 5.33$ & 1.15 & $4395 / 4130$ & $0.98 / 0.44 / 1.20$ & 5,58 \\
\hline $02-000$ & FV Tau/c AB & M2.5/3.5 & 20 & $3.25 / 7.00$ & 0.49 & $3412 / 3155$ & $0.18 / 0.06 / 0.21$ & $5,20,58$ \\
\hline $02-016$ & KPNO-Tau 13 & M5 & 34 & $\ldots$ & 0.70 & 3125 & 0.150 & 34 \\
\hline $02-000$ & DG Tau B & $\ldots$ & $\ldots$ & $\ldots$ & $\ldots$ & 4000 & 5.500 & 10 \\
\hline $02-022$ & DG Tau A & K6 & 5 & 1.41 & 0.36 & 4205 & 1.700 & 5,58 \\
\hline $02-000$ & KPNO-Tau 4 & M9.5 & 5,18 & 2.45 & 0.00 & 2500 & 0.004 & 5,18 \\
\hline $02-000$ & IRAS $04248+2612 \mathrm{AB}$ & M2-5.5 & 5,59 & 7.02 & 1.51 & 2845 & 0.270 & 5,59 \\
\hline $15-020$ & JH 507 & M4 & 5 & 0.76 & 0.29 & 3270 & 0.420 & 5,27 \\
\hline 13-004 & GV Tau AB & K3-7 & 27,59 & 12.10 & $\ldots$ & 4000 & 1.820 & 59 \\
\hline $13-000$ & IRAS 04264+2433 & M1 & 59 & 10.40 & & 3605 & 0.140 & 59 \\
\hline $15-040$ & DH Tau AB & M1 & 5 & 1.25 & 0.32 & 3705 & 0.560 & 5,27 \\
\hline $15-042$ & DI Tau AB & M0 & 5 & 0.76 & 0.43 & 3850 & 0.990 & 5,27 \\
\hline $15-044$ & KPNO-Tau 5 & M7.5 & 5,19 & 0.00 & 0.00 & 2783 & 0.023 & 19 \\
\hline $14-006$ & IQ Tau A & M0.5 & 5 & 1.25 & 0.45 & 3778 & 0.880 & 5,27 \\
\hline $13-000$ & CFHT-Tau 20 & M5.5 & 18 & 3.60 & & 3065 & 0.138 & 18 \\
\hline $14-000$ & KPNO-Tau 6 & M9.0 & 18 & 0.88 & 0.00 & 2571 & 0.003 & 5,18 \\
\hline $13-035$ & FX Tau AB & M1 & 27 & 1.08 & 0.31 & 3720 & 1.020 & 27 \\
\hline $14-057$ & DK Tau AB & K7 & 5 & 0.76 & 0.28 & 4060 & 1.300 & 5,27 \\
\hline $14-000$ & KPNO-Tau 7 & M8.25 & 5 & $\ldots$ & 0.00 & 2632 & 0.003 & 5 \\
\hline $22-013$ & MHO 9 & M4.25 & 5 & 1.73 & 0.56 & 3234 & 0.220 & 5,4 \\
\hline $22-021$ & MHO 4 & M7.1 & 5,18 & 1.10 & 0.14 & 2880 & 0.048 & 5,58 \\
\hline $22-040$ & L1551 IRS5 & & $\ldots$ & $\ldots$ & $\ldots$ & 4800 & 2.600 & 10 \\
\hline $22-042$ & LkHa 358 & M5.5 & 5 & 13.60 & 3.84 & 3058 & 0.590 & 5,45 \\
\hline $22-000$ & HH 30 & M0 & 59 & 2.96 & $\cdots$ & 3800 & 0.006 & 59 \\
\hline $22-043$ & HL Tau & K5 & 59 & 7.43 & $\ldots$ & 4395 & 1.530 & 59 \\
\hline $22-047$ & XZ Tau AB & M2/M3.5 & 20 & $1.40 / 1.35$ & 0.11 & $3561 / 3345$ & $0.17 / 0.31 / 0.33$ & 5,20 \\
\hline $22-056$ & L1551 NE & & $\ldots$ & $\cdots$ & & $\begin{array}{cc}\cdots \\
0\end{array}$ & $\ldots$ & $\ldots$ \\
\hline 03-005 & HK Tau AB & $\mathrm{M} 0.5 / \mathrm{M} 2$ & 5 & 2.32 & 0.97 & $3778 / 3560$ & 0.560 & 5,27 \\
\hline $22-070$ & V710 Tau BA & M0.5/M2 & 5 & $1.80 / 1.82$ & 0.62 & $3778 / 3560$ & $0.58 / 0.52 / 1.10$ & 5,58 \\
\hline 19-009 & JH 665 & M5.5 & 5 & 0.97 & 0.54 & 3058 & 0.260 & 5,27 \\
\hline $22-089$ & L1551 51 & K7 & 5 & 0.00 & 0.00 & 4060 & 0.470 & 5,27 \\
\hline $22-097$ & V827 Tau & K7 & 5 & 0.28 & 0.31 & 4060 & 1.100 & 5,27 \\
\hline 03-016 & Haro 6-13 & M0 & 59 & 11.90 & $\ldots$ & 3800 & 2.110 & 59 \\
\hline
\end{tabular}


Table 9. continued.

\begin{tabular}{|c|c|c|c|c|c|c|c|c|}
\hline$\overline{\mathrm{XEST}}$ & Name & $\operatorname{Spec}^{a}$ & Refs. & $\begin{array}{r}A_{\mathrm{V}}^{a} \\
(\mathrm{mag})\end{array}$ & $\begin{array}{r}A_{J} \\
(\mathrm{mag})\end{array}$ & $\begin{array}{l}T_{\text {eff }}^{a} \\
(\mathrm{~K})\end{array}$ & $\begin{array}{r}L_{*}^{b} \\
\left(L_{\odot}\right)\end{array}$ & Refs. \\
\hline $22-100$ & V826 Tau & K7 & 5 & 0.28 & 0.19 & 4060 & 0.940 & 5,27 \\
\hline $22-101$ & MHO 5 & M6 & 5 & 0.23 & 0.17 & 2990 & 0.110 & 5,58 \\
\hline 03-017 & CFHT-Tau 7 & M5.75 & 66 & 0.00 & $\ldots$ & 3030 & 0.061 & 18,19 \\
\hline 03-019 & V928 Tau AB & M0.5 & 5 & 1.87 & 1.04 & 3778 & 1.400 & 5,27 \\
\hline 03-022 & FY Tau & K5 & 5 & 3.47 & 1.07 & 4350 & 1.000 & 5,27 \\
\hline $03-023$ & FZ Tau & M0 & 5 & 2.72 & 0.99 & 3850 & 0.980 & 5,58 \\
\hline $17-002$ & IRAS 04295+2251 & $\ldots$ & $\ldots$ & $\ldots$ & $\ldots$ & 3400 & 1.200 & 10 \\
\hline 19-049 & $\mathrm{UZ}$ Tau $\mathrm{E}+\mathrm{W}(\mathrm{AB})$ & $\mathrm{M} 1 / 2 / 3$ & 27,20 & $1.49 / 0.83$ & 0.26 & $3705 / 3560$ & $0.40 / 0.49 / 0.89$ & 5,27 \\
\hline 17-009 & JH 112 & K6 & 27 & 3.23 & 0.93 & 4205 & 0.740 & 27 \\
\hline 03-031 & CFHT-Tau 5 & M7.5 & 18 & 9.22 & $\ldots$ & 2783 & 0.075 & 18 \\
\hline 04-003 & CFHT-Tau 5 & M7.5 & 18 & 9.22 & & 2783 & 0.075 & 18 \\
\hline 03-035 & MHO 8 & M6 & 5 & 0.19 & 0.28 & 2990 & 0.170 & 5,4 \\
\hline 04-009 & MHO 8 & M6 & 5 & 0.19 & 0.28 & 2990 & 0.170 & 5,4 \\
\hline 04-010 & GH Tau AB & $\mathrm{M} 1.5 / \mathrm{M} 2$ & 58 & $0.69 / 0.64$ & 0.11 & $3631 / 3556$ & $0.38 / 0.34 / 0.81$ & 5,58 \\
\hline 04-012 & V807 Tau SNab & K7/M3 & 58 & $0.36 / 0.36$ & 0.04 & $3999 / 3388$ & $1.07 / 0.32 / 2.10$ & 5,58 \\
\hline $18-004$ & KPNO-Tau 14 & M6 & 34 & $\ldots$ & 0.85 & 2990 & 0.110 & 34 \\
\hline $17-000$ & CFHT-Tau 12 & M6 & 66 & 3.44 & $\ldots$ & 2995 & 0.036 & 18,19 \\
\hline 04-016 & V830 Tau & K7 & 5 & 0.28 & 0.13 & 4060 & 0.780 & 5,27 \\
\hline $18-000$ & IRAS S04301+261 & M0 & 5 & $\ldots$ & 1.76 & 3850 & 0.025 & 5 \\
\hline $17-000$ & IRAS $04302+2247$ & $\ldots$ & $\ldots$ & $\ldots$ & $\ldots$ & $\ldots$ & 0.300 & 13 \\
\hline $17-027$ & IRAS $04303+2240$ & M0.5 & 59 & 11.70 & $\ldots$ & 3700 & 2.200 & 59 \\
\hline $04-034$ & GI Tau & K7 & 5 & 0.87 & 0.43 & 4060 & 1.000 & 5,27 \\
\hline 04-035 & GK Tau AB & K7 & 5 & 0.87 & 0.46 & 4060 & 1.400 & 5,27 \\
\hline 18-019 & IS Tau AB & K7/M4.5 & 58 & $3.67 / 3.67$ & 0.95 & $3999 / 3221$ & $0.50 / 0.19 / 0.66$ & 5,58 \\
\hline $17-058$ & CI Tau & K7 & 27 & 1.77 & 0.51 & 4060 & 0.870 & 27 \\
\hline $18-030$ & IT Tau AB & $\mathrm{K} 2$ & 5 & 4.09 & 1.86 & 4900 & 2.400 & 5,27 \\
\hline $17-066$ & JH 108 & M1 & 27 & 1.46 & 0.42 & 3720 & 0.300 & 27 \\
\hline $17-068$ & CFHT-BD Tau 1 & M7.1 & 39,19 & 3.10 & & 2853 & 0.017 & 39,19 \\
\hline $25-026$ & AA Tau & K7 & 5 & 0.49 & 0.27 & 4060 & 0.800 & 5,27 \\
\hline 09-010 & HO Tau AB & M0.5 & 5 & 1.11 & 0.46 & 3778 & 0.170 & 5,27 \\
\hline 08-019 & FF Tau AB & K7 & 5 & 2.22 & 0.51 & 4060 & 0.690 & 5,27 \\
\hline $12-040$ & DN Tau & M0 & 5 & 0.49 & 0.34 & 3850 & 1.000 & 5,27 \\
\hline $12-000$ & IRAS $04325+2402 \mathrm{AB}$ & & $\ldots$ & $\ldots$ & & & 0.720 & 28 \\
\hline 12-059 & CoKu Tau 3 AB & M1 & 5 & 3.26 & 2.15 & 3705 & 0.980 & 5,27 \\
\hline $09-022$ & KPNO-Tau 8 & M5.75 & 5 & $\ldots$ & 0.14 & 3024 & 0.021 & 5 \\
\hline 08-037 & HQ Tau AB & $\ldots$ & $\ldots$ & $\ldots$ & $\ldots$ & $\ldots$ & $\ldots$ & $\ldots$ \\
\hline 09-026 & HQ Tau AB & $\ldots$ & $\ldots$ & $\ldots$ & $\ldots$ & $\ldots$ & $\ldots$ & $\ldots$ \\
\hline $08-043$ & KPNO-Tau 15 & M2.75 & 34 & $\ldots$ & 0.56 & 3451 & 0.140 & 34 \\
\hline 09-031 & KPNO-Tau 15 & M2.75 & 34 & $\ldots$ & 0.56 & 3451 & 0.140 & 34 \\
\hline 08-000 & KPNO-Tau 9 & M8.5 & 5 & $\ldots$ & 0.00 & 2555 & 0.001 & 5 \\
\hline 09-000 & KPNO-Tau 9 & M8.5 & 5 & $\ldots$ & 0.00 & 2555 & 0.001 & 5 \\
\hline 08-048 & HP Tau AB & K3 & 5 & 2.26 & 0.91 & 4730 & 1.400 & 5,27 \\
\hline 08-051a & HP Tau/G3 AB & K7 & 5 & 2.32 & 0.77 & 4060 & 0.710 & 5,27 \\
\hline 08-051 & HP Tau/G2 & G0 & 5 & 2.08 & 0.66 & 6030 & 6.500 & 5,27 \\
\hline $08-058$ & Haro 6-28 AB & M2/M3.5 & 20 & $2.30 / 1.90$ & 1.34 & $3556 / 3342$ & $0.09 / 0.03 / 0.12$ & 5,20 \\
\hline 08-000 & CFHT-BD Tau 2 & M7.5 & 5 & 0.00 & 0.56 & 2795 & 0.011 & 5,39 \\
\hline 08-080 & CFHT-BD Tau 3 & M7.75 & 5 & 0.00 & 0.28 & 2747 & 0.007 & 5,39 \\
\hline 05-005 & CFHT-Tau 6 & M7.25 & 33,18 & 0.41 & 0.07 & 2818 & 0.024 & 33,18 \\
\hline $05-000$ & IRAS $04361+2547$ & & $\ldots$ & $\ldots$ & $\ldots$ & $\ldots$ & 3.700 & 13 \\
\hline 05-013 & GN Tau AB & M2.5 & 33 & $\ldots$ & 1.17 & 3488 & 0.720 & 33 \\
\hline 05-017 & IRAS $04365+2535$ & $\ldots$ & $\ldots$ & $\ldots$ & $\ldots$ & $\ldots$ & 2.200 & $27(\mathrm{Lb})^{c}$ \\
\hline 05-024 & IRAS $04369+2539$ & $\mathrm{~K} 4$ & 59 & 18.10 & & 4580 & 20.700 & 59 \\
\hline 07-011 & JH 223 & M2 & 27 & $\ldots$ & 0.14 & 3560 & 0.180 & 33 \\
\hline 07-022 & Haro 6-32 & M5 & 33 & $\ldots$ & 0.17 & 3125 & 0.120 & 33 \\
\hline 07-000 & ITG 33 A & M3 & 33 & 3.50 & 1.89 & 3415 & 0.051 & 33,35 \\
\hline 07-000 & CFHT-Tau 8 & M5.5 & 33 & 1.77 & 0.70 & 3058 & 0.024 & $33,18,19$ \\
\hline 07-000 & IRAS $04381+2540$ & $\ldots$ & $\ldots$ & $\ldots$ & $\ldots$ & $\ldots$ & 0.700 & 13 \\
\hline 07-041 & IRAS $04385+2550 \mathrm{AB}$ & M0 & 33 & 10.20 & 1.13 & 3850 & 0.180 & 33,59 \\
\hline $10-017$ & CoKuLk332/G2 AB & M0.5/2.5 & 20 & $2.00 / 3.30$ & 0.99 & $3778 / 3485$ & $0.24 / 0.15 / 1.10$ & 33,20 \\
\hline
\end{tabular}


Table 9. continued.

\begin{tabular}{|c|c|c|c|c|c|c|c|c|}
\hline XEST & Name & $\operatorname{Spec}^{a}$ & Refs. & $\begin{array}{r}A_{\mathrm{V}}^{a} \\
(\mathrm{mag}) \\
\end{array}$ & $\begin{array}{r}A_{J} \\
(\mathrm{mag}) \\
\end{array}$ & $\begin{array}{l}T_{\text {eff }}^{a} \\
(\mathrm{~K}) \\
\end{array}$ & $\begin{array}{r}L_{*}^{b} \\
\left(L_{\odot}\right)\end{array}$ & Refs. \\
\hline $10-018$ & CoKuLk332/G1 AB & K7/M1 & 58 & $4.13 / 4.13$ & 1.33 & $3707 / 3945$ & $0.85 / 0.58 / 1.70$ & 33,58 \\
\hline $10-020$ & V955 Tau AB & K5/M1 & 58 & $3.72 / 3.72$ & 0.90 & $4395 / 3715$ & $0.32 / 0.18 / 1.00$ & 33,58 \\
\hline $10-034$ & CIDA 7 & M4.75 & 66 & $\ldots$ & $\ldots$ & $\ldots$ & 0.050 & $27(\mathrm{Lb})^{c}$ \\
\hline $10-045$ & DP Tau & M0.5 & 27 & 1.46 & 0.41 & 3778 & 0.200 & 33,27 \\
\hline $10-060$ & GO Tau & M0 & 27 & 1.18 & 0.77 & 3850 & 0.370 & 33,27 \\
\hline $26-012$ & 2M J04552333+30 & M6.25 & 33,19 & 0.00 & 0.00 & 2959 & 0.015 & 33,19 \\
\hline $26-034$ & $2 \mathrm{M} \mathrm{J} 04554046+30$ & M5.25 & 33 & $\ldots$ & 0.07 & 3091 & 0.021 & 33 \\
\hline $26-043$ & $\mathrm{AB}$ Aur & B9.5-A0 & 27,7 & 0.25 & 0.24 & 10050 & 49.000 & $33,27,8,9$ \\
\hline $26-050$ & 2MJ04554757/801 & M4.75/5.6 & 33 & & 0.00 & $3161 / 3044$ & $0.10 / 0.01 / 0.11$ & 33 \\
\hline $26-067$ & SU Aur & $\mathrm{G} 2$ & 27 & 0.90 & 0.21 & 5860 & 9.900 & 33,27 \\
\hline $26-072$ & НBC 427 & K7 & 27 & 0.00 & 0.17 & 4350 & 1.100 & 33,27 \\
\hline \multicolumn{9}{|c|}{ Additional sources from Chandra } \\
\hline C1-0 & KPNO-Tau 10 & M5 & 34 & $\ldots$ & 0.14 & 3125 & 0.052 & 34 \\
\hline C1-1 & IRAS $04158+2805$ & M3 & 5 & 8.63 & $\ldots$ & 2760 & 0.050 & 59 \\
\hline C2-1 & Haro 6-5 B & K5 & 59 & 9.96 & $\ldots$ & 4395 & 0.047 & 59 \\
\hline $\mathrm{C} 2-2$ & FS Tau AC & M0/M3.5 & 20 & $4.95 / 5.15$ & 0.53 & $3876 / 3345$ & $0.15 / 0.17 / 0.32$ & 27,20 \\
\hline C3-1 & FV Tau/c AB & M2.5/3.5 & 20 & $3.25 / 7.00$ & 0.49 & $3412 / 3155$ & $0.18 / 0.06 / 0.21$ & $5,20,58$ \\
\hline C3-2 & DG Tau B & & & & $\ldots$ & 4000 & 5.500 & 10 \\
\hline C4-1 & GV Tau AB & K3-7 & 27,59 & 12.10 & $\ldots$ & 4000 & 1.820 & 59 \\
\hline C5-2 & HN Tau AB & K5/M4 & 5 & $1.18 / 0.91$ & 0.12 & $4395 / 3273$ & $0.22 / 0.03 / 0.25$ & 5,58 \\
\hline C5-1 & L1551 55 & K7 & 5 & 0.69 & 0.20 & 4060 & 0.370 & 5,27 \\
\hline C5-4 & HD 28867 & B9IVn & 65 & 0.25 & $\ldots$ & 10500 & $64.90 / 62.10 / 127.00$ & 65 \\
\hline C5-3 & DM Tau & M1 & 5 & 0.59 & 0.31 & 3705 & 0.300 & 5,58 \\
\hline C6-1 & CFHT-BD Tau 4 & M7 & 33 & 2.60 & 0.70 & 2853 & 0.062 & $33,39,19$ \\
\hline C6-0 & L1527 IRS & & .. & .. & $\ldots$ & $\ldots$ & 1.300 & $27(\mathrm{Lb})^{c}$ \\
\hline C6-0 & CFHT-Tau 17 & M5.5 & 66 & 6.50 & $\ldots$ & 3030 & 0.068 & 18 \\
\hline C6-2 & IRAS $04370+2559$ & $\ldots$ & $\ldots$ & $\ldots$ & $\ldots$ & $\ldots$ & 0.210 & $27(\mathrm{Lb})^{c}$ \\
\hline
\end{tabular}

${ }^{a}$ For multiples, first number or spectral type refers to primary, second to secondary component.

${ }^{b}$ For multiples, three numbers give primary/secondary/total system luminosity.

${ }^{c}$ Referring to $L_{\mathrm{bol}}$ as derived from integration of the optical and infrared spectrum.

Notes on individual objects:

- V773 Tau = XEST-20-042: Multiple entries refer to A and C, respectively

- HD $28867=$ C5-4: total $L_{*}$ (Berghoefer et al. 1996) scaled to $d=140 \mathrm{pc}$, split to components according to $V$ mag (Walter et al. 2003).

- FQ Tau = XEST-23-067 = XEST-24-058, FS Tau = XEST-11-057, XZ Tau = XEST-22-047, CoKuLk332/G2 = XEST-10-017: $T_{\text {eff }}$ of components read from Fig. 10 in Ref. 20. 
Table 10. Fundamental parameters of targets in XEST (4): Age, mass, radius, rotation.

\begin{tabular}{|c|c|c|c|c|c|c|c|c|c|}
\hline XEST & Name & $\begin{array}{l}\text { Age }^{a, b} \\
\text { (Myr) }\end{array}$ & $\begin{array}{l}\operatorname{Mass}^{a, c} \\
\left(M_{\odot}\right) \\
\end{array}$ & Refs. & $\begin{array}{l}\text { Radius }^{d} \\
\left(R_{\odot}\right)\end{array}$ & $\begin{array}{r}P \\
\text { (d) }\end{array}$ & Refs. & $\begin{array}{r}v \sin i \\
\left(\mathrm{~km} \mathrm{~s}^{-1}\right) \\
\end{array}$ & Refs. \\
\hline $27-115$ & HBC 352 & $\ldots$ & 1.05 & 2 & 0.79 & $<0.53$ & $\mathrm{C}$ & $>75.00$ & 48 \\
\hline $27-000$ & HBC 353 & $\ldots$ & $\ldots$ & $\ldots$ & 0.71 & $<4.08$ & $\mathrm{C}$ & 8.80 & 2 \\
\hline 06-005 & HBC 358 AB & 3.26 & 0.41 & 27 & 1.38 & $\ldots$ & $\mathrm{C}$ & $<10.00$ & 2 \\
\hline 06-007 & HBC 359 & 3.54 & 0.41 & 27 & 1.33 & $\ldots$ & $\mathrm{C}$ & $<10.00$ & 48 \\
\hline 06-059 & L1489 IRS & 0.80 & 1.45 & 10 & 3.65 & $<4.02$ & $\mathrm{C}$ & 46.00 & 10 \\
\hline $20-001$ & LkCa 1 & 0.87 & 0.27 & 5 & 1.93 & $<4.18$ & $\mathrm{C}$ & 23.30 & 48 \\
\hline $20-005$ & Anon 1 & 0.50 & 0.56 & 5 & 3.63 & $\ldots$ & $\ldots$ & $\ldots$ & $\ldots$ \\
\hline $20-000$ & IRAS $04108+2803 \mathrm{~A}$ & $\ldots$ & $\ldots$ & $\ldots$ & $\ldots$ & $\ldots$ & $\ldots$ & $\ldots$ & $\ldots$ \\
\hline $20-022$ & IRAS $04108+2803$ B & 1.60 & 0.36 & 10 & 1.72 & $<6.23$ & $\mathrm{C}$ & 14.00 & 10 \\
\hline $20-000$ & 2M J04141188+28 & $\ldots$ & 0.08 & 46 & 0.47 & & $\ldots$ & $\ldots$ & $\ldots$ \\
\hline $20-042$ & V773 Tau ABC & $6.35 / 0.93$ & $1.53 / 0.57$ & 58 & 1.91 & 3.43 & 48 & 55.00 & 48 \\
\hline $20-043$ & FM Tau & 2.76 & 0.57 & 5 & 1.53 & & $\ldots$ & . & $\ldots$ \\
\hline $20-046$ & CW Tau & 6.97 & 1.40 & 27 & 1.57 & 8.25 & 48 & 27.40 & 48 \\
\hline $20-047$ & CIDA 1 & & $\ldots$ & $\ldots$ & $\ldots$ & $\ldots$ & $\ldots$ & 5.30 & 48 \\
\hline $20-056$ & MHO 2/1 & $1.54 / 4.85$ & $0.36 / 0.34$ & 5 & 1.78 & $<4.19$ & $\mathrm{C}$ & 21.50 & 59 \\
\hline $20-058$ & MHO 3 & 2.0 & 0.75 & 5 & 1.93 & $\ldots$ & $\ldots$ & $\ldots$ & $\ldots$ \\
\hline $20-069$ & FO Tau AB & $1.45 / 1.54$ & $0.38 / 0.38$ & 58 & 1.83 & $\ldots$ & $\ldots$ & $\ldots$ & $\ldots$ \\
\hline $20-073$ & CIDA 2 & 0.39 & 0.20 & 5 & 2.02 & $\ldots$ & $\ldots$ & $\ldots$ & $\ldots$ \\
\hline 23-002 & CY Tau & 1.53 & 0.42 & 5 & 1.79 & 7.50 & 48 & 10.00 & 48 \\
\hline $24-002$ & CY Tau & 1.53 & 0.42 & 5 & 1.79 & 7.50 & 48 & 10.00 & 48 \\
\hline $23-004$ & LkCa 5 & 2.31 & 0.40 & 5 & 1.60 & $<2.19$ & $\mathrm{C}$ & 37.00 & 48 \\
\hline 24-004 & LkCa 5 & 2.31 & 0.40 & 5 & 1.60 & $<2.19$ & $\mathrm{C}$ & 37.00 & 48 \\
\hline $23-008$ & CIDA 3 & 3.34 & 0.24 & 5 & 1.17 & $\ldots$ & $\ldots$ & $\ldots$ & $\ldots$ \\
\hline $24-008$ & CIDA 3 & 3.34 & 0.24 & 5 & 1.17 & $\ldots$ & $\ldots$ & $\ldots$ & $\ldots$ \\
\hline 23-015 & V410 X3 & 2.68 & 0.12 & 5 & 1.08 & $<3.79$ & $\mathrm{C}$ & 14.10 & 48 \\
\hline 24-015 & V410 X3 & 2.68 & 0.12 & 5 & 1.08 & $<3.79$ & $\mathrm{C}$ & 14.10 & 48 \\
\hline $23-018$ & V410 A13 & $\ldots$ & 0.10 & 58 & 0.72 & $<3.72$ & $\mathrm{C}$ & 9.80 & 45 \\
\hline $24-000$ & V410 A13 & $\ldots$ & 0.10 & 58 & 0.72 & $<3.72$ & $\mathrm{C}$ & 9.80 & 45 \\
\hline $23-000$ & V410 A24 & 18.7 & 1.22 & 5 & 1.58 & $\ldots$ & $\ldots$ & $\ldots$ & $\ldots$ \\
\hline $24-000$ & V410 A24 & 18.7 & 1.22 & 5 & 1.58 & $\ldots$ & $\ldots$ & $\ldots$ & $\ldots$ \\
\hline $23-029$ & V410 A25 & 0.64 & 0.46 & 5 & 3.08 & $\ldots$ & $\ldots$ & $\ldots$ & $\ldots$ \\
\hline 24-027 & V410 A25 & 0.64 & 0.46 & 5 & 3.08 & $\ldots$ & $\ldots$ & $\ldots$ & $\ldots$ \\
\hline $23-032$ & V410 Tau ABC & $2.74 / 5.22$ & $1.51 / 0.12$ & 58 & 2.31 & 1.94 & 48 & 71.00 & 48 \\
\hline $24-028$ & V410 Tau ABC & $2.74 / 5.22$ & $1.51 / 0.12$ & 58 & 2.31 & 1.94 & 48 & 71.00 & 48 \\
\hline $23-033$ & DD Tau AB & $4.47 / 4.47$ & $0.30 / 0.30$ & 58 & 1.03 & $\ldots$ & $\ldots$ & $\ldots$ & $\ldots$ \\
\hline 24-029 & DD Tau AB & $4.47 / 4.47$ & $0.30 / 0.30$ & 58 & 1.03 & $\ldots$ & $\ldots$ & $\ldots$ & $\ldots$ \\
\hline $23-035$ & CZ Tau AB & 2.10 & 0.32 & 5 & 1.49 & $\ldots$ & $\ldots$ & $\ldots$ & $\ldots$ \\
\hline 24-030 & CZ Tau AB & 2.10 & 0.32 & 5 & 1.49 & $\ldots$ & $\ldots$ & $\ldots$ & $\ldots$ \\
\hline $23-036$ & IRAS $04154+2823$ & 5.30 & 0.33 & 5 & 0.99 & $\ldots$ & $\ldots$ & $\ldots$ & $\ldots$ \\
\hline 24-031 & IRAS $04154+2823$ & 5.30 & 0.33 & 5 & 0.99 & $\ldots$ & $\ldots$ & $\ldots$ & $\ldots$ \\
\hline $23-037$ & V410 X2 & 0.44 & 0.56 & 5 & 3.90 & $\ldots$ & $\ldots$ & $\ldots$ & $\ldots$ \\
\hline 24-032 & V410 X2 & 0.44 & 0.56 & 5 & 3.90 & $\ldots$ & $\ldots$ & $\ldots$ & $\ldots$ \\
\hline $23-045$ & V410 X4 & 0.06 & 0.27 & 5 & 3.56 & $\ldots$ & $\ldots$ & $\ldots$ & $\ldots$ \\
\hline 24-038 & V410 X4 & 0.06 & 0.27 & 5 & 3.56 & $\ldots$ & $\ldots$ & $\ldots$ & $\ldots$ \\
\hline $23-047$ & V892 Tau & 2.97 & 2.89 & 5 & 2.66 & $\ldots$ & $\ldots$ & $\ldots$ & $\ldots$ \\
\hline 24-040 & V892 Tau & 2.97 & 2.89 & 5 & 2.66 & $\ldots$ & $\ldots$ & $\ldots$ & $\ldots$ \\
\hline $23-048$ & LR 1 & 17.5 & 1.00 & 5 & 1.15 & $\ldots$ & $\ldots$ & $\ldots$ & $\ldots$ \\
\hline 24-000 & LR 1 & 17.5 & 1.00 & 5 & 1.15 & $\ldots$ & $\ldots$ & $\ldots$ & $\ldots$ \\
\hline $23-050$ & V410 X7 & 1.90 & 0.50 & 5 & 1.69 & $\ldots$ & $\ldots$ & $\ldots$ & $\ldots$ \\
\hline 24-042 & V410 X7 & 1.90 & 0.50 & 5 & 1.69 & $\ldots$ & $\ldots$ & $\ldots$ & $\ldots$ \\
\hline $23-000$ & V410 A20 & 20.7 & 1.01 & 5 & 1.06 & $\ldots$ & $\ldots$ & $\ldots$ & $\ldots$ \\
\hline $24-000$ & V410 A20 & 20.7 & 1.01 & 5 & 1.06 & $\ldots$ & $\ldots$ & $\ldots$ & $\ldots$ \\
\hline $23-056$ & Hubble 4 & 0.68 & 0.74 & 5 & 3.33 & $<13.17$ & $\mathrm{C}$ & 12.80 & 48 \\
\hline 24-047 & Hubble 4 & 0.68 & 0.74 & 5 & 3.33 & $<13.17$ & $\mathrm{C}$ & 12.80 & 48 \\
\hline $23-000$ & KPNO-Tau 2 & $\ldots$ & 0.05 & 46 & 0.34 & $\ldots$ & $\ldots$ & $\ldots$ & $\ldots$ \\
\hline $24-000$ & KPNO-Tau 2 & $\ldots$ & 0.05 & 46 & 0.34 & $\ldots$ & $\ldots$ & $\ldots$ & $\ldots$ \\
\hline $23-000$ & CoKu Tau 1 & 29.1 & 0.70 & 59 & 0.81 & $<2.67$ & $\mathrm{C}$ & 15.30 & 59 \\
\hline $24-000$ & CoKu Tau 1 & 29.1 & 0.70 & 59 & 0.81 & $<2.67$ & $\mathrm{C}$ & 15.30 & 59 \\
\hline 23-061 & V410 X6 & 0.71 & 0.18 & 5 & 1.60 & $\ldots$ & $\ldots$ & $\ldots$ & $\ldots$ \\
\hline
\end{tabular}


Table 10. continued.

\begin{tabular}{|c|c|c|c|c|c|c|c|c|c|}
\hline$\overline{\mathrm{XEST}}$ & Name & $\begin{array}{l}\text { Age }^{a, b} \\
\text { (Myr) }\end{array}$ & $\begin{array}{l}\operatorname{Mass}^{a, c} \\
\left(M_{\odot}\right)\end{array}$ & Refs. & $\begin{array}{l}\text { Radius }^{d} \\
\left(R_{\odot}\right)\end{array}$ & $\begin{array}{r}P \\
\text { (d) }\end{array}$ & Refs. & $\begin{array}{r}v \sin i \\
\left(\mathrm{~km} \mathrm{~s}^{-1}\right)\end{array}$ & Refs. \\
\hline $24-054$ & V410 X6 & 0.71 & 0.18 & 5 & 1.60 & $\ldots$ & $\ldots$ & $\ldots$ & $\ldots$ \\
\hline $23-063$ & V410 X5 & 4.21 & 0.14 & 5 & 1.03 & $\ldots$ & $\ldots$ & $\ldots$ & $\ldots$ \\
\hline $24-055$ & V410 X5 & 4.21 & 0.14 & 5 & 1.03 & $\ldots$ & $\ldots$ & $\ldots$ & $\ldots$ \\
\hline $23-067$ & FQ Tau AB & $2.82 / 1.89$ & $0.31 / 0.29$ & 20 & 1.40 & $\ldots$ & $\ldots$ & $\ldots$ & $\ldots$ \\
\hline $24-058$ & FQ Tau AB & $2.82 / 1.89$ & $0.31 / 0.29$ & 20 & 1.40 & $\ldots$ & $\ldots$ & $\ldots$ & $\ldots$ \\
\hline $28-100$ & BP Tau & 1.91 & 0.75 & 27 & 1.97 & 7.60 & 48 & 7.80 & 48 \\
\hline $23-074$ & V819 Tau AB & 2.02 & 0.76 & 5 & 1.93 & 5.60 & 48 & $<15.00$ & 48 \\
\hline 24-061 & V819 Tau AB & 2.02 & 0.76 & 5 & 1.93 & 5.60 & 48 & $<15.00$ & 48 \\
\hline $16-000$ & IRAS 04166+2706 & $\ldots$ & $\ldots$ & $\ldots$ & $\ldots$ & $\ldots$ & $\ldots$ & $\ldots$ & $\ldots$ \\
\hline $16-000$ & IRAS 04169+2702 & $\ldots$ & $\ldots$ & $\ldots$ & $\ldots$ & $\ldots$ & $\ldots$ & $\ldots$ & $\ldots$ \\
\hline $11-000$ & CFHT-Tau 19 & $\ldots$ & $\ldots$ & $\ldots$ & 0.93 & $\ldots$ & $\ldots$ & $\ldots$ & $\ldots$ \\
\hline $11-000$ & IRAS $04181+2655$ & 0.90 & 0.68 & 10 & 2.80 & $<4.29$ & $\mathrm{C}$ & 33.00 & 10 \\
\hline $11-000$ & IRAS $04181+2654 \mathrm{AB}$ & & $\ldots$ & $\ldots$ & $\ldots$ & $\ldots$ & $\ldots$ & $\ldots$ & $\ldots$ \\
\hline $11-023$ & 2M J04213459 & 4.56 & 0.13 & 33 & 0.91 & $\ldots$ & $\ldots$ & $\ldots$ & $\ldots$ \\
\hline $01-028$ & IRAS 04187+1927 & $\ldots$ & $\ldots$ & $\ldots$ & $\ldots$ & $\ldots$ & $\ldots$ & $\ldots$ & $\ldots$ \\
\hline $11-037$ & CFHT-Tau 10 & $\ldots$ & $\ldots$ & $\ldots$ & 0.52 & $\ldots$ & $\ldots$ & $\ldots$ & $\ldots$ \\
\hline $11-000$ & 2M J04215450+2652 & $\ldots$ & $\ldots$ & $\ldots$ & 0.26 & $\ldots$ & $\ldots$ & $\ldots$ & $\ldots$ \\
\hline $21-038$ & RY Tau & 2.11 & 2.37 & 27 & 3.57 & 5.60 & 48 & 52.20 & 48 \\
\hline 21-039 & HD 283572 & 7.92 & 1.70 & 27 & 2.56 & 1.55 & 48 & 95.00 & 48 \\
\hline $01-045$ & T Tau N(+Sab) & 2.67 & 2.41 & 27 & 3.62 & 2.80 & 48 & 20.10 & 48 \\
\hline $11-054$ & Haro 6-5 B & $\ldots$ & $\ldots$ & $\ldots$ & 0.37 & $<0.91$ & $\mathrm{C}$ & 20.70 & 59 \\
\hline $11-057$ & FS Tau AC & $17.2 / 3.06$ & $0.61 / 0.28$ & 20 & 0.93 & $\ldots$ & $\ldots$ & $\ldots$ & $\ldots$ \\
\hline 21-044 & LkCa 21 & 1.16 & 0.35 & 27 & 2.18 & 8.80 & 48 & 60.00 & 48 \\
\hline $01-054$ & RX J0422.1+1934 & $\ldots$ & $\ldots$ & $\ldots$ & $\ldots$ & $\ldots$ & $\ldots$ & $\ldots$ & $\ldots$ \\
\hline 01-062 & 2M J04221332+1934 & $\ldots$ & $\ldots$ & $\ldots$ & 0.59 & $\ldots$ & $\ldots$ & $\ldots$ & $\ldots$ \\
\hline $11-079$ & CFHT-Tau 21 & $\ldots$ & $\ldots$ & $\ldots$ & 1.53 & $\ldots$ & $\ldots$ & $\ldots$ & $\ldots$ \\
\hline 02-013 & FV Tau AB & $4.75 / 7.65$ & $1.19 / 0.88$ & 58 & 1.71 & $\ldots$ & $\ldots$ & $\ldots$ & $\ldots$ \\
\hline $02-000$ & FV Tau/c AB & $3.14 / 5.40$ & $0.31 / 0.16$ & 20,58 & 1.22 & $\ldots$ & $\ldots$ & $\ldots$ & $\ldots$ \\
\hline 02-016 & KPNO-Tau 13 & 2.66 & 0.19 & 34 & 1.32 & $\ldots$ & $\ldots$ & $\ldots$ & $\ldots$ \\
\hline $02-000$ & DG Tau B & 0.30 & 0.72 & 10 & 4.90 & $\ldots$ & $\ldots$ & $\ldots$ & $\ldots$ \\
\hline $02-022$ & DG Tau A & 1.32 & 0.91 & 5 & 2.46 & 6.30 & 48 & 21.70 & 48 \\
\hline $02-000$ & KPNO-Tau 4 & $\ldots$ & 0.01 & 46 & 0.35 & $\ldots$ & $\ldots$ & $\ldots$ & $\ldots$ \\
\hline $02-000$ & IRAS $04248+2612 \mathrm{AB}$ & 16.5 & 0.34 & 5 & 2.14 & $<6.61$ & $\mathrm{C}$ & 16.40 & 59 \\
\hline $15-020$ & JH 507 & 1.33 & 0.27 & 5 & 2.02 & & $\ldots$ & & $\ldots$ \\
\hline $13-004$ & GV Tau AB & 0.89 & 0.68 & 59 & 2.82 & $<5.63$ & $\mathrm{C}$ & 25.30 & 59 \\
\hline $13-000$ & IRAS $04264+2433$ & 7.95 & 0.41 & 59 & 0.96 & $<1.52$ & $\mathrm{C}$ & 32.00 & 10 \\
\hline $15-040$ & DH Tau AB & 1.53 & 0.47 & 5 & 1.82 & 7.00 & 48 & 10.00 & 48 \\
\hline $15-042$ & DI Tau AB & 1.07 & 0.56 & 5 & 2.24 & 7.70 & 48 & 10.50 & 48 \\
\hline $15-044$ & KPNO-Tau 5 & $\ldots$ & 0.04 & 46 & 0.65 & & $\ldots$ & $\ldots$ & $\ldots$ \\
\hline 14-006 & IQ Tau A & 1.06 & 0.51 & 5 & 2.20 & 6.25 & 48 & 11.50 & 48 \\
\hline $13-000$ & CFHT-Tau 20 & $\ldots$ & $\ldots$ & $\ldots$ & 1.31 & $\ldots$ & $\ldots$ & $\ldots$ & $\ldots$ \\
\hline $14-000$ & KPNO-Tau 6 & $\ldots$ & 0.03 & 46 & 0.28 & $\ldots$ & $\ldots$ & $\ldots$ & $\ldots$ \\
\hline $13-035$ & FX Tau AB & 0.90 & 0.47 & 27 & 2.44 & $<12.34$ & $\mathrm{C}$ & 10.00 & 48 \\
\hline $14-057$ & DK Tau AB & 1.32 & 0.74 & 5 & 2.31 & 8.40 & 48 & 11.40 & 48 \\
\hline $14-000$ & KPNO-Tau 7 & $\ldots$ & 0.03 & 46 & 0.28 & $\ldots$ & $\ldots$ & $\ldots$ & $\ldots$ \\
\hline $22-013$ & MHO 9 & 2.33 & 0.24 & 5 & 1.50 & $<7.66$ & $\mathrm{C}$ & 9.90 & 48 \\
\hline $22-021$ & MHO 4 & $\ldots$ & 0.10 & 58 & 0.88 & $<6.29$ & $\mathrm{C}$ & 7.10 & 48 \\
\hline $22-040$ & L1551 IRS5 & 4.80 & 1.58 & 10 & 2.34 & $<3.82$ & $\mathrm{C}$ & 31.00 & 10 \\
\hline $22-042$ & LkHa 358 & 0.12 & 0.21 & 5 & 2.74 & $<6.80$ & $\mathrm{C}$ & 20.40 & 45 \\
\hline $22-000$ & HH 30 & & 0.52 & 59 & 0.19 & & & $<12.00$ & 59 \\
\hline $22-043$ & HL Tau & 2.45 & 1.20 & 59 & 2.13 & $<7.21$ & $\mathrm{C}$ & 15.00 & 48 \\
\hline $22-047$ & XZ Tau AB & $4.58 / 1.79$ & $0.37 / 0.29$ & 20 & 1.18 & 2.60 & 48 & $\ldots$ & $\ldots$ \\
\hline $22-056$ & L1551 NE & $\ldots$ & $\ldots$ & $\ldots$ & $\ldots$ & $\ldots$ & $\ldots$ & $\ldots$ & $\ldots$ \\
\hline 03-005 & HK Tau AB & 1.76 & 0.51 & 5 & 1.75 & $<8.86$ & $\mathrm{C}$ & 10.00 & 48 \\
\hline $22-070$ & V710 Tau BA & $1.69 / 1.69$ & $0.51 / 0.40$ & 5 & 1.78 & $<5.67$ & $\mathrm{C}$ & 15.90 & 48 \\
\hline 19-009 & JH 665 & 0.60 & 0.19 & 5 & 1.82 & $\ldots$ & $\ldots$ & & $\ldots$ \\
\hline $22-089$ & L1551 51 & 5.42 & 0.80 & 5 & 1.39 & 2.43 & 48 & 27.00 & 48 \\
\hline 22-097 & V827 Tau & 1.59 & 0.75 & 5 & 2.13 & 3.75 & 48 & 18.50 & 48 \\
\hline 03-016 & Haro 6-13 & 0.57 & 0.52 & 59 & 3.36 & $<7.30$ & $\mathrm{C}$ & 23.30 & 59 \\
\hline
\end{tabular}


Table 10. continued.

\begin{tabular}{|c|c|c|c|c|c|c|c|c|c|}
\hline$\overline{\mathrm{XEST}}$ & Name & $\begin{array}{l}\text { Age }^{a, b} \\
\text { (Myr) }\end{array}$ & $\begin{array}{l}\text { Mass }^{a, c} \\
\left(M_{\odot}\right)\end{array}$ & Refs. & $\begin{array}{l}\text { Radius }^{d} \\
\left(R_{\odot}\right)\end{array}$ & $\begin{array}{r}P \\
(\mathrm{~d}) \\
\end{array}$ & Refs. & $\begin{array}{r}v \sin i \\
\left(\mathrm{~km} \mathrm{~s}^{-1}\right) \\
\end{array}$ & Refs. \\
\hline $22-100$ & V826 Tau & 1.94 & 0.75 & 5 & 1.96 & 3.70 & 48 & 4.20 & 48 \\
\hline $22-101$ & MHO 5 & $\ldots$ & 0.13 & 58 & 1.24 & $<7.84$ & $\mathrm{C}$ & 8.00 & 48 \\
\hline 03-017 & CFHT-Tau 7 & $\ldots$ & $\ldots$ & $\ldots$ & 0.89 & & $\ldots$ & $\ldots$ & $\ldots$ \\
\hline 03-019 & V928 Tau AB & 0.73 & 0.50 & 5 & 2.77 & $<7.45$ & $\mathrm{C}$ & 18.80 & 48 \\
\hline $03-022$ & FY Tau & 3.98 & 1.13 & 5 & 1.77 & $\ldots$ & $\ldots$ & $\ldots$ & $\ldots$ \\
\hline $03-023$ & FZ Tau & 1.08 & 0.56 & 5 & 2.23 & $\ldots$ & $\ldots$ & $\ldots$ & $\ldots$ \\
\hline $17-002$ & IRAS $04295+2251$ & 1.12 & 0.56 & 27 & 1.49 & $<1.48$ & $\mathrm{C}$ & 51.00 & 10 \\
\hline $19-049$ & UZ Tau E+W(AB) & $2.26 / 1.43$ & $0.47 / 0.39$ & 5 & 1.54 & $<4.90$ & $\mathrm{C}$ & 15.90 & 48 \\
\hline $17-009$ & JH 112 & 4.06 & 0.95 & 27 & 1.62 & $\ldots$ & $\ldots$ & $\ldots$ & $\ldots$ \\
\hline 03-031 & CFHT-Tau 5 & $\ldots$ & $\ldots$ & $\ldots$ & 1.18 & $\ldots$ & $\ldots$ & $\ldots$ & $\ldots$ \\
\hline 04-003 & CFHT-Tau 5 & $\ldots$ & $\ldots$ & $\ldots$ & 1.18 & $\ldots$ & $\ldots$ & $\ldots$ & $\ldots$ \\
\hline 03-035 & MHO 8 & 0.47 & 0.15 & 5 & 1.54 & $<4.67$ & $\mathrm{C}$ & 16.70 & 45 \\
\hline 04-009 & MHO 8 & 0.47 & 0.15 & 5 & 1.54 & $<4.67$ & $\mathrm{C}$ & 16.70 & 45 \\
\hline 04-010 & GH Tau AB & $2.04 / 2.03$ & $0.42 / 0.38$ & 58 & 1.56 & $<3.57$ & $\mathrm{C}$ & 22.10 & 48 \\
\hline 04-012 & V807 Tau SNab & $1.51 / 1.74$ & $0.69 / 0.31$ & 58 & 2.16 & $\ldots$ & $\ldots$ & $\ldots$ & $\ldots$ \\
\hline $18-004$ & KPNO-Tau 14 & 1.09 & 0.13 & 34 & 1.24 & $\ldots$ & $\ldots$ & $\ldots$ & $\ldots$ \\
\hline $17-000$ & CFHT-Tau 12 & $\ldots$ & $\ldots$ & $\ldots$ & 0.70 & $\ldots$ & $\ldots$ & $\ldots$ & $\ldots$ \\
\hline 04-016 & V830 Tau & 2.48 & 0.76 & 5 & 1.79 & 2.75 & 48 & 29.10 & 48 \\
\hline $18-000$ & IRAS S04301+261 & $\ldots$ & $\ldots$ & $\ldots$ & 0.36 & $\ldots$ & $\ldots$ & $\ldots$ & $\ldots$ \\
\hline $17-000$ & IRAS $04302+2247$ & $\ldots$ & 0.34 & 59 & $\ldots$ & $\ldots$ & $\ldots$ & $\ldots$ & $\ldots$ \\
\hline $17-027$ & IRAS $04303+2240$ & 0.50 & 0.51 & 59 & 3.62 & $<5.23$ & $\mathrm{C}$ & 35.00 & 59 \\
\hline 04-034 & GI Tau & 1.79 & 0.75 & 5 & 2.03 & 7.20 & 48 & 11.20 & 48 \\
\hline 04-035 & GK Tau AB & 1.22 & 0.74 & 5 & 1.97 & 4.60 & 48 & 18.70 & 48 \\
\hline $18-019$ & IS Tau AB & $4.15 / 2.52$ & $0.72 / 0.24$ & 58 & 1.48 & $\ldots$ & $\ldots$ & $\ldots$ & $\ldots$ \\
\hline $17-058$ & CI Tau & 2.15 & 0.76 & 27 & 1.89 & $<9.20$ & $\mathrm{C}$ & 10.40 & 48 \\
\hline $18-030$ & IT Tau AB & 4.75 & 1.67 & 5 & 2.16 & $\ldots$ & $\ldots$ & $\ldots$ & $\ldots$ \\
\hline $17-066$ & JH 108 & 3.40 & 0.48 & 27 & 1.32 & $\ldots$ & $\ldots$ & $\ldots$ & $\ldots$ \\
\hline $17-068$ & CFHT-BD Tau 1 & $\ldots$ & $\ldots$ & $\ldots$ & 0.53 & $\ldots$ & $\ldots$ & $\ldots$ & $\ldots$ \\
\hline $25-026$ & AA Tau & 2.40 & 0.76 & 5 & 1.81 & 8.22 & 48 & 11.40 & 48 \\
\hline 09-010 & HO Tau AB & 9.13 & 0.52 & 5 & 0.96 & $\ldots$ & $\ldots$ & $\ldots$ & $\ldots$ \\
\hline 08-019 & FF Tau AB & 2.95 & 0.77 & 5 & 1.68 & $\ldots$ & $\ldots$ & $\ldots$ & $\ldots$ \\
\hline $12-040$ & DN Tau & 1.05 & 0.56 & 5 & 2.25 & 6.30 & 48 & 8.10 & 48 \\
\hline $12-000$ & IRAS $04325+2402 \mathrm{AB}$ & $\ldots$ & $\ldots$ & $\ldots$ & $\ldots$ & $\ldots$ & $\ldots$ & $\ldots$ & $\ldots$ \\
\hline $12-059$ & CoKu Tau 3 AB & 0.92 & 0.46 & 5 & 2.41 & $\ldots$ & $\ldots$ & $\ldots$ & $\ldots$ \\
\hline 09-022 & KPNO-Tau 8 & $\ldots$ & $\ldots$ & $\ldots$ & 0.52 & $\ldots$ & $\ldots$ & $\ldots$ & $\ldots$ \\
\hline $08-037$ & HQ Tau AB & ... & $\ldots$ & $\ldots$ & $\cdots$ & $\ldots$ & $\ldots$ & $\ldots$ & $\ldots$ \\
\hline 09-026 & HQ Tau AB & $\ldots$ & $\ldots$ & $\ldots$ & $\ldots$ & $\ldots$ & $\ldots$ & $\ldots$ & $\ldots$ \\
\hline $08-043$ & KPNO-Tau 15 & 3.86 & 0.32 & 34 & 1.05 & $\ldots$ & $\ldots$ & $\ldots$ & $\ldots$ \\
\hline 09-031 & KPNO-Tau 15 & 3.86 & 0.32 & 34 & 1.05 & $\ldots$ & $\ldots$ & $\ldots$ & $\ldots$ \\
\hline 08-000 & KPNO-Tau 9 & $\ldots$ & $\ldots$ & $\ldots$ & 0.19 & $\ldots$ & $\ldots$ & $\ldots$ & $\ldots$ \\
\hline 09-000 & KPNO-Tau 9 & $\ldots$ & $\ldots$ & $\ldots$ & 0.19 & $\ldots$ & $\ldots$ & $\ldots$ & $\ldots$ \\
\hline 08-048 & HP Tau AB & 6.90 & 1.39 & 5 & 1.77 & 5.90 & 48 & 15.40 & 48 \\
\hline 08-051a & HP Tau/G3 AB & 2.83 & 0.77 & 5 & 1.71 & $\ldots$ & $\ldots$ & $\ldots$ & $\ldots$ \\
\hline $08-051$ & HP Tau/G2 & 10.5 & 1.58 & 5 & 2.34 & 1.20 & 48 & 100.00 & 48 \\
\hline 08-058 & Haro 6-28 AB & $10.0 / 18.5$ & $0.35 / 0.21$ & 20 & 0.79 & $<3.98$ & $\mathrm{C}$ & 10.10 & 59 \\
\hline 08-000 & CFHT-BD Tau 2 & $\ldots$ & $\ldots$ & $\ldots$ & 0.45 & $\ldots$ & $\ldots$ & $\ldots$ & $\ldots$ \\
\hline 08-080 & CFHT-BD Tau 3 & $\ldots$ & 0.04 & 46 & 0.38 & $\ldots$ & $\ldots$ & $\ldots$ & $\ldots$ \\
\hline 05-005 & CFHT-Tau 6 & $\ldots$ & $\ldots$ & $\ldots$ & 0.65 & $\ldots$ & $\ldots$ & $\cdots$ & $\cdots$ \\
\hline $05-000$ & IRAS $04361+2547$ & $\ldots$ & $\ldots$ & $\ldots$ & $\ldots$ & $\ldots$ & $\ldots$ & $\ldots$ & $\ldots$ \\
\hline $05-013$ & GN Tau AB & 1.03 & 0.36 & 33 & 2.33 & $<11.80$ & $\mathrm{C}$ & 10.00 & 48 \\
\hline 05-017 & IRAS $04365+2535$ & $\ldots$ & $\ldots$ & $\ldots$ & $\ldots$ & $\ldots$ & $\ldots$ & & $\ldots$ \\
\hline $05-024$ & IRAS 04369+2539 & $\ldots$ & $\ldots$ & $\ldots$ & 7.24 & $\ldots$ & $\ldots$ & $<15.00$ & 59 \\
\hline 07-011 & JH 223 & 4.27 & 0.37 & 33 & 1.12 & $\ldots$ & $\ldots$ & $\ldots$ & $\ldots$ \\
\hline $07-022$ & Haro 6-32 & 3.33 & 0.09 & 33 & 1.18 & $\ldots$ & $\ldots$ & $\ldots$ & $\ldots$ \\
\hline $07-000$ & ITG 33 A & 12.9 & 0.26 & 33 & 0.65 & $\ldots$ & $\ldots$ & $\ldots$ & $\ldots$ \\
\hline $07-000$ & CFHT-Tau 8 & 6.93 & 0.10 & 33 & 0.55 & $\ldots$ & $\ldots$ & $\ldots$ & $\ldots$ \\
\hline $07-000$ & IRAS 04381+2540 & & $\ldots$ & $\ldots$ & $\ldots$ & $\ldots$ & $\ldots$ & $\ldots$ & $\ldots$ \\
\hline $07-041$ & IRAS $04385+2550 \mathrm{AB}$ & 12.2 & 0.59 & 33 & 0.96 & $<2.13$ & $\mathrm{C}$ & 22.80 & 59 \\
\hline $10-017$ & CoKuLk332/G2 AB & $5.60 / 4.50$ & $0.52 / 0.34$ & 20 & 0.99 & $<2.25$ & $\mathrm{C}$ & 22.30 & 48 \\
\hline
\end{tabular}


Table 10. continued.

\begin{tabular}{|c|c|c|c|c|c|c|c|c|c|}
\hline$\overline{\mathrm{XEST}}$ & Name & $\begin{array}{l}\text { Age }^{a, b} \\
\text { (Myr) }\end{array}$ & $\begin{array}{l}\text { Mass }^{a, c} \\
\left(M_{\odot}\right)\end{array}$ & Refs. & $\begin{array}{l}\text { Radius }^{d} \\
\left(R_{\odot}\right)\end{array}$ & $\begin{array}{r}P \\
\text { (d) }\end{array}$ & Refs. & $\begin{array}{r}v \sin i \\
\left(\mathrm{~km} \mathrm{~s}^{-1}\right)\end{array}$ & Refs. \\
\hline $10-018$ & CoKuLk332/G1 AB & $1.02 / 2.76$ & $0.46 / 0.66$ & 58 & 2.24 & & $\cdots$ & & $\cdots$ \\
\hline $10-020$ & V955 Tau AB & $24.1 / 6.70$ & $0.90 / 0.47$ & 58 & 0.98 & $<4.90$ & $\mathrm{C}$ & 10.10 & 48 \\
\hline $10-034$ & CIDA 7 & & 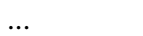 & $\ldots$ & $\ldots$ & $\ldots$ & $\ldots$ & & $\ldots$ \\
\hline $10-045$ & DP Tau & 7.24 & 0.52 & 33 & 1.05 & $<2.76$ & $\mathrm{C}$ & 19.20 & 59 \\
\hline $10-060$ & GO Tau & 3.78 & 0.58 & 33 & 1.37 & $<3.96$ & $\mathrm{C}$ & 17.50 & 48 \\
\hline $26-012$ & 2M J04552333+30 & $\ldots$ & $\ldots$ & $\ldots$ & 0.47 & $\ldots$ & $\ldots$ & $\ldots$ & $\ldots$ \\
\hline $26-034$ & 2M J04554046+30 & 9.39 & 0.11 & 33 & 0.51 & $\ldots$ & $\ldots$ & $\ldots$ & $\ldots$ \\
\hline $26-043$ & $\mathrm{AB}$ Aur & 4.00 & 2.70 & 9 & 2.31 & $<1.46$ & $\mathrm{C}$ & 80.00 & 6 \\
\hline $26-050$ & 2MJ04554757/801 & 3.82 & 0.07 & 33 & 1.05 & & $\ldots$ & & $\ldots$ \\
\hline $26-067$ & SU Aur & 6.02 & 1.91 & 33 & 3.06 & 1.70 & 48 & 65.00 & 48 \\
\hline $26-072$ & HBC 427 & 3.43 & 1.13 & 33 & 1.85 & 9.30 & 48 & 10.40 & 2 \\
\hline \multicolumn{10}{|c|}{ Additional sources from Chandra } \\
\hline $\mathrm{C} 1-0$ & KPNO-Tau 10 & 5.7 & 0.14 & 34 & 0.78 & & 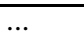 & & 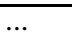 \\
\hline C1-1 & IRAS $04158+2805$ & 1.55 & 0.35 & 27 & 0.98 & $<2.15$ & $\mathrm{C}$ & 23.10 & 59 \\
\hline C2-1 & Haro 6-5 B & $\ldots$ & $\ldots$ & $\ldots$ & 0.37 & $<0.91$ & $\mathrm{C}$ & 20.70 & 59 \\
\hline $\mathrm{C} 2-2$ & FS Tau AC & $17.2 / 3.06$ & $0.61 / 0.28$ & 20 & 0.93 & $\ldots$ & $\ldots$ & $\ldots$ & $\ldots$ \\
\hline C3-1 & $\mathrm{FV} \mathrm{Tau} / \mathrm{c} \mathrm{AB}$ & $3.14 / 5.40$ & $0.31 / 0.16$ & 20,58 & 1.22 & $\ldots$ & $\ldots$ & $\ldots$ & $\ldots$ \\
\hline C3-2 & DG Tau B & 0.30 & 0.72 & 10 & 4.90 & $\ldots$ & $\ldots$ & $\ldots$ & $\ldots$ \\
\hline C4-1 & GV Tau AB & 0.89 & 0.68 & 59 & 2.82 & $<5.63$ & $\mathrm{C}$ & 25.30 & 59 \\
\hline C5-2 & HN Tau AB & $41 / 14$ & $0.78 / 0.07$ & 58 & 0.81 & $<0.78$ & $\mathrm{C}$ & 52.80 & 48 \\
\hline C5-1 & L1551 55 & 8.2 & 0.81 & 5 & 1.23 & 6.20 & 48 & $<10.00$ & 48 \\
\hline C5-4 & HD 28867 & $3.71 / 3.76$ & $2.85 / 2.82$ & 65 & 2.60 & $<2.00$ & 65 & 65.00 & 65 \\
\hline C5-3 & DM Tau & 3.26 & 0.47 & 5 & 1.33 & $<6.74$ & $\mathrm{C}$ & 10.00 & 48 \\
\hline C6-1 & CFHT-BD Tau 4 & $\ldots$ & 0.06 & 46 & 1.02 & $\ldots$ & $\ldots$ & $\ldots$ & $\ldots$ \\
\hline C6-0 & L1527 IRS & $\ldots$ & $\ldots$ & $\ldots$ & $\ldots$ & $\ldots$ & $\ldots$ & $\ldots$ & $\ldots$ \\
\hline C6-0 & CFHT-Tau 17 & $\ldots$ & $\ldots$ & $\ldots$ & 0.94 & $\ldots$ & $\ldots$ & $\ldots$ & $\ldots$ \\
\hline C6-2 & IRAS $04370+2559$ & $\ldots$ & $\ldots$ & $\ldots$ & $\ldots$ & $\ldots$ & $\ldots$ & $\ldots$ & $\ldots$ \\
\hline
\end{tabular}

${ }^{a}$ For binaries, first number refers to primary, second to secondary component (calculated from $L_{*}$ and $T_{\text {eff }}$ ).

${ }^{b}$ Ages derived after Siess et al. (2000) using the same principal parameters as for masses, quoted in Table 9).

${ }^{c}$ Masses derived after Siess et al. (2000) using principal parameters quoted in Table 9.

Exceptions quoted directly from literature: HBC 352 (XEST-27-115; ref. 2), 2M J04141188+28 (XEST-20-000; ref. 46), V410 A13 (XEST23-018 = XEST-24-000; ref. 58), KPNO-Tau 2 (XEST-23-000 = XEST-24-000; ref. 46), KPNO-Tau 4 (XEST-02-000; ref. 46), KPNO-Tau 5 (XEST-15-044; ref. 46), KPNO-Tau 6 (XEST-14-000; ref. 46), KPNO-Tau 7 (XEST-14-000; ref. 46), MHO 4 (XEST-22-021; ref. 58), HH 30 (XEST-22-000; ref. 59), IRAS 04302+2247 (XEST17-000; ref. 59), CFHT-BD Tau 3 (XEST-08-080; ref. 46), CFHT-BD Tau 4 (C6-1; ref. 46).

${ }^{d}$ For multiples, radius is given only for primary if luminosity of primary is explicitly known.

Notes on individual objects:

- Mass/age calculations for references different from those of $T_{\text {eff }}$ and $L_{*}$ for CW Tau (XEST-20-046; ref. 27 instead of 5), and IRAS 04158+2805 (C1-1; ref. 27 instead of 59).

- V773 Tau = XEST-20-042: Multiple entries refer to A and C, respectively.

- V410 Tau = XEST-23-032 = XEST-24-028: Multiple entries refer to A and C, respectively.

- HD 28867 = C5-4: radius and $P$ of G-type companion after Walter et al. (2003). 
M. Güdel et al.: XMM-Newton extended survey of Taurus, Online Material p 32

Table 11. Fundamental parameters of targets in XEST (5); Accretion and evolution.

\begin{tabular}{|c|c|c|c|c|c|c|c|c|c|}
\hline XEST & Name & $\begin{array}{r}\dot{M}(\min / \max )^{a} \\
\left(M_{\odot} \mathrm{yr}^{-1}\right)\end{array}$ & Refs. & $\begin{array}{r}E W(\mathrm{H} \alpha)^{b} \\
(\AA)\end{array}$ & $\begin{array}{l}\text { TTS } \\
\text { type }\end{array}$ & Refs. $^{c}$ & $\begin{array}{l}\mathrm{IR}^{d} \\
\text { class }\end{array}$ & Refs. $^{d}$ & Type \\
\hline $27-115$ & HBC 352 & $\ldots$ & $\ldots$ & 0 & $\mathrm{~W}$ & 29 & III & 1,27 & 3 \\
\hline $27-000$ & HBC 353 & $\ldots$ & $\ldots$ & 0 & W & 29 & III & 27 & 3 \\
\hline 06-005 & HBC 358 AB & $<8.97$ & 20 & $4-10$ & $\mathrm{~W} / \mathrm{W}$ & 37,29 & III & 1,27 & 3 \\
\hline 06-007 & НBC 359 & & $\ldots$ & $2-9$ & W & 56,37 & III & 27 & 3 \\
\hline $06-059$ & L1489 IRS & -7.15 & 59 & $41-56$ & $\mathrm{C}^{*}$ & 59,29 & I & $1,59,27$ & 1 \\
\hline $20-001$ & LkCa 1 & $<-9.72$ & 58 & $3-4$ & W & 29,45 & III & 1,27 & 3 \\
\hline $20-005$ & Anon 1 & $<-8.94$ & 58 & $1-3$ & W & 37,29 & III & 1,27 & 3 \\
\hline $20-000$ & IRAS $04108+2803 \mathrm{~A}$ & $\ldots$ & $\ldots$ & 37 & $\mathrm{C}$ & 29 & II & 27 & 2 \\
\hline $20-022$ & IRAS 04108+2803 B & $\ldots$ & $\ldots$ & $\ldots$ & $\ldots$ & $\ldots$ & I & 59,27 & 1 \\
\hline $20-000$ & 2M J04141188+28 & -10.00 & 46 & 250 & $\mathrm{C}$ & 46 & $\ldots$ & $\ldots$ & 4 \\
\hline $20-042$ & V773 Tau ABC & $<-10.00$ & 58 & $2-4$ & W & 21,24 & II & 1,27 & 3 \\
\hline $20-043$ & FM Tau & $-8.87 /-8.45$ & 58,44 & $51-101$ & $\mathrm{C}$ & 21,29 & II & 1,27 & 2 \\
\hline $20-046$ & CW Tau & -7.99 & 58 & $135-140$ & $\mathrm{C}$ & 7,29 & II & $1,59,27$ & 2 \\
\hline $20-047$ & CIDA 1 & -8.50 & 57 & $112-149$ & $\mathrm{C}$ & 41,29 & II & 27 & 2 \\
\hline $20-056$ & MHO 2/1 & $<-8.48$ & 59 & $58-88$ & $\mathrm{C} / \mathrm{C}$ & 59,4 & $\mathrm{I} ; \mathrm{II}$ & $59 ; 22$ & 2 \\
\hline $20-058$ & MHO 3 & & & $16-21$ & $\mathrm{C}$ & 4 & II & 22 & 2 \\
\hline $20-069$ & FO Tau AB & $-7.90 /-7.58$ & 58,20 & $116-137$ & $\mathrm{C}$ & 29,20 & II & 1,27 & 2 \\
\hline $20-073$ & CIDA 2 & & & $5-7$ & W & 3,29 & III & 1,27 & 3 \\
\hline $23-002$ & CY Tau & $-8.86 /-8.12$ & 58 & $55-70$ & $\mathrm{C}$ & 58,7 & II & 1,27 & 2 \\
\hline $24-002$ & CY Tau & $-8.86 /-8.12$ & 58,44 & $55-70$ & $\mathrm{C}$ & 58,7 & II & 1,27 & 2 \\
\hline 23-004 & LkCa 5 & $<-10.00$ & 58 & 4 & W & 29 & III & 1,27 & 3 \\
\hline 24-004 & LkCa 5 & $<-10.00$ & 58 & 4 & W & 29 & III & 1,27 & 3 \\
\hline 23-008 & CIDA 3 & $\ldots$ & $\ldots$ & 6 & W & 3 & II & 1,27 & 3 \\
\hline $24-008$ & CIDA 3 & $\ldots$ & $\ldots$ & 6 & W & 3 & II & 1,27 & 3 \\
\hline $23-015$ & V410 X3 & $<-9.30$ & 57 & $14-30$ & $\mathrm{~W}$ ? & 41,57 & $\ldots$ & $\ldots$ & 3 \\
\hline 24-015 & V410 X3 & $<-9.30$ & 57 & $14-30$ & W? & 41,57 & $\ldots$ & $\ldots$ & 3 \\
\hline $23-018$ & V410 A13 & -11.30 & 45 & $27-41$ & $\mathrm{C} ?$ & 45,58 & II & 22 & 2 \\
\hline $24-000$ & V410 A13 & -11.30 & 45 & $27-41$ & C? & 45,58 & II & 22 & 2 \\
\hline $23-000$ & V410 A24 & $\ldots$ & $\ldots$ & $\ldots$ & $\ldots$ & $\ldots$ & $\ldots$ & $\ldots$ & 9 \\
\hline $24-000$ & V410 A24 & $\ldots$ & $\ldots$ & $\ldots$ & $\ldots$ & $\ldots$ & $\ldots$ & $\ldots$ & 9 \\
\hline $23-029$ & V410 A25 & $\ldots$ & $\ldots$ & $\ldots$ & $\ldots$ & $\ldots$ & $\ldots$ & $\ldots$ & 9 \\
\hline $24-027$ & V410 A25 & $\ldots$ & $\ldots$ & $\ldots$ & $\ldots$ & $\ldots$ & $\ldots$ & $\ldots$ & 9 \\
\hline $23-032$ & V410 Tau ABC & $<-8.80$ & 58 & $2-3$ & W & 29,7 & III & 1,27 & 3 \\
\hline $24-028$ & V410 Tau ABC & $<-8.80$ & 58 & $2-3$ & W & 29,7 & III & 1,27 & 3 \\
\hline $23-033$ & DD Tau AB & $-9.10 /-7.21$ & 58,20 & 90-206 & $\mathrm{C}$ & 37,20 & II & 1,27 & 2 \\
\hline 24-029 & DD Tau AB & $-9.10 /-7.21$ & 58,20 & 90-206 & $\mathrm{C}$ & 37,20 & II & 1,27 & 2 \\
\hline $23-035$ & CZ Tau AB & $\ldots$ & $\ldots$ & 4-7 & $\mathrm{W}$ & 7,29 & II & 1,27 & 3 \\
\hline 24-030 & CZ Tau AB & $\ldots$ & $\ldots$ & $4-7$ & W & 7,29 & II & 1,27 & 3 \\
\hline $23-036$ & IRAS $04154+2823$ & $\ldots$ & $\ldots$ & $>17$ & $\mathrm{C}$ & 59 & FS;II & $1 ; 59,27$ & 2 \\
\hline 24-031 & IRAS 04154+2823 & $\ldots$ & $\ldots$ & $>17$ & $\mathrm{C}$ & 59 & FS;II & $1 ; 59,27$ & 2 \\
\hline $23-037$ & V410 X2 & $\ldots$ & $\ldots$ & $\ldots$ & $\ldots$ & $\ldots$ & $\ldots$ & $\ldots$ & 9 \\
\hline $24-032$ & V410 X2 & $\ldots$ & $\ldots$ & $\ldots$ & $\ldots$ & $\ldots$ & $\ldots$ & $\ldots$ & 9 \\
\hline 23-045 & V410 X4 & $\ldots$ & $\ldots$ & $\ldots$ & $\ldots$ & $\ldots$ & $\ldots$ & $\ldots$ & 9 \\
\hline $24-038$ & V410 X4 & $\ldots$ & $\ldots$ & $\ldots$ & $\ldots$ & $\ldots$ & $\ldots$ & $\ldots$ & 9 \\
\hline $23-047$ & V892 Tau & $\ldots$ & $\ldots$ & $7-13$ & $\mathrm{Ae}$ & 29,7 & II & 1,27 & 5 \\
\hline $24-040$ & V892 Tau & $\ldots$ & $\ldots$ & $7-13$ & $\mathrm{Ae}$ & 29,7 & II & 1,27 & 5 \\
\hline $23-048$ & LR 1 & $\ldots$ & $\ldots$ & $\ldots$ & $\ldots$ & $\ldots$ & $\ldots$ & $\ldots$ & 9 \\
\hline $24-000$ & LR 1 & $\ldots$ & $\ldots$ & $\ldots$ & $\ldots$ & $\ldots$ & $\ldots$ & $\ldots$ & 9 \\
\hline $23-050$ & V410 X7 & $\ldots$ & $\ldots$ & 3 & W & 58 & III & 22 & 3 \\
\hline $24-042$ & V410 X7 & $\ldots$ & $\ldots$ & 3 & W & 58 & III & 22 & 3 \\
\hline $23-000$ & V410 A20 & $\ldots$ & $\ldots$ & $\ldots$ & $\ldots$ & $\ldots$ & $\ldots$ & $\ldots$ & 9 \\
\hline $24-000$ & V410 A20 & $\ldots$ & $\ldots$ & $\ldots$ & $\ldots$ & $\ldots$ & $\ldots$ & $\ldots$ & 9 \\
\hline 23-056 & Hubble 4 & $<-8.16$ & 58 & $3-4$ & W & 7,29 & III & 1,27 & 3 \\
\hline $24-047$ & Hubble 4 & $<-8.16$ & 58 & $3-4$ & $\mathrm{~W}$ & 7,29 & III & 1,27 & 3 \\
\hline $23-000$ & KPNO-Tau 2 & $<-12.00$ & 46 & $4-12$ & $\mathrm{~W}$ & 46,5 & $\ldots$ & $\ldots$ & 4 \\
\hline 24-000 & KPNO-Tau 2 & $<-12.00$ & 46 & 4-12 & W & 46,5 & $\ldots$ & $\ldots$ & 4 \\
\hline $23-000$ & CoKu Tau 1 & -7.36 & 59 & $70-111$ & $\mathrm{C}$ & 59,29 & II & $59,1,27$ & 2 \\
\hline $24-000$ & CoKu Tau 1 & -7.36 & 59 & $70-111$ & $\mathrm{C}$ & 59,29 & II & $59,1,27$ & 2 \\
\hline $23-061$ & V410 X6 & $\ldots$ & $\ldots$ & 13 & $\mathrm{~W}$ & 38 & & $\ldots$ & 3 \\
\hline
\end{tabular}


Table 11. continued.

\begin{tabular}{|c|c|c|c|c|c|c|c|c|c|}
\hline XEST & Name & $\begin{array}{r}\dot{M}(\min / \max )^{a} \\
\left(M_{\odot} \mathrm{yr}^{-1}\right)\end{array}$ & Refs. & $\begin{array}{r}E W(\mathrm{H} \alpha)^{b} \\
(\AA)\end{array}$ & $\begin{array}{l}\text { TTS } \\
\text { type }\end{array}$ & Refs. $^{c}$ & $\begin{array}{l}\mathrm{IR}^{d} \\
\text { class }\end{array}$ & Refs. $^{d}$ & Type \\
\hline $24-054$ & V410 X6 & $\ldots$ & $\ldots$ & 13 & $\mathrm{~W}$ & 38 & $\ldots$ & $\ldots$ & 3 \\
\hline $23-063$ & V410 X5 & $\ldots$ & $\ldots$ & $11-19$ & W? & 58,45 & II & 22 & 3 \\
\hline $24-055$ & V410 X5 & $\ldots$ & $\ldots$ & $11-19$ & W? & 58,45 & II & 22 & 3 \\
\hline $23-067$ & FQ Tau AB & $-7.98 /-6.45$ & 20,44 & $81-114$ & $\mathrm{C} / \mathrm{C}$ & 29,7 & II & 1,27 & 2 \\
\hline $24-058$ & FQ Tau AB & $-7.98 /-6.45$ & 20,44 & $81-114$ & $\mathrm{C} / \mathrm{C}$ & 29,7 & II & 1,27 & 2 \\
\hline $28-100$ & $\mathrm{BP} \mathrm{Tau}$ & $-7.88 /-7.54$ & 58,44 & $40-92$ & $\mathrm{C}$ & 55,29 & II & 1,27 & 2 \\
\hline $23-074$ & V819 Tau AB & $<-8.86$ & 58 & $2-3$ & W & 60,29 & III & 1,27 & 3 \\
\hline 24-061 & V819 Tau AB & $<-8.86$ & 58 & $2-3$ & W & 60,29 & III & 1,27 & 3 \\
\hline $16-000$ & IRAS $04166+2706$ & $\ldots$ & $\ldots$ & $\ldots$ & $\ldots$ & $\ldots$ & I & $59,1,27$ & 1 \\
\hline $16-000$ & IRAS $04169+2702$ & $\ldots$ & $\ldots$ & $\ldots$ & $\ldots$ & $\ldots$ & I & $59,1,27$ & 1 \\
\hline $11-000$ & CFHT-Tau 19 & $\ldots$ & $\ldots$ & 442 & $\mathrm{C}$ & 18 & $\ldots$ & $\ldots$ & 2 \\
\hline $11-000$ & IRAS 04181+2655 & $\ldots$ & $\ldots$ & $\ldots$ & $\ldots$ & $\ldots$ & I & 59,27 & 1 \\
\hline $11-000$ & IRAS $04181+2654 \mathrm{AB}$ & $\ldots$ & $\ldots$ & $\ldots$ & $\ldots$ & $\ldots$ & I & 59,27 & 1 \\
\hline $11-023$ & 2M J04213459 & $\ldots$ & $\ldots$ & $\ldots$ & W & 33 & $\ldots$ & $\ldots$ & 3 \\
\hline $01-028$ & IRAS $04187+1927$ & $\ldots$ & $\ldots$ & $\ldots$ & $\ldots$ & $\ldots$ & II & 27 & 2 \\
\hline $11-037$ & CFHT-Tau 10 & $\ldots$ & $\ldots$ & 17 & W & 18 & $\ldots$ & $\ldots$ & 3 \\
\hline $11-000$ & $2 \mathrm{M} \mathrm{J} 04215450+2652$ & $\ldots$ & $\ldots$ & $\ldots$ & $\ldots$ & $\ldots$ & $\ldots$ & $\ldots$ & 4 \\
\hline $21-038$ & RY Tau & $-7.19 /-7.04$ & 63 & 13 & $\mathrm{C}$ & 29 & II & 1,27 & 2 \\
\hline $21-039$ & HD 283572 & & $\ldots$ & 0 & $\mathrm{~W}$ & 29 & III & 1,27 & 3 \\
\hline $01-045$ & T Tau N(+Sab) & $-7.50 /-7.24$ & 58,63 & 41 & $\mathrm{C}$ & 29 & II & $59,1,27$ & 2 \\
\hline $11-054$ & Haro 6-5 B & -6.76 & 59 & 91 & $\mathrm{C}^{*}$ & 59 & (II) & (59) & 1 \\
\hline $11-057$ & FS Tau AC & $-9.50 /-8.09$ & 58,44 & $57-81$ & $\mathrm{C} / \mathrm{C}$ & 7,58 & FS;II & $1 ; 27$ & 2 \\
\hline 21-044 & LkCa 21 & $\ldots$ & $\ldots$ & 6 & W & 37 & III & 1,27 & 3 \\
\hline $01-054$ & RX J0422.1+1934 & $\ldots$ & $\ldots$ & 19 & $\mathrm{~W}$ & 36 & $\ldots$ & $\ldots$ & 3 \\
\hline $01-062$ & $2 \mathrm{M} \mathrm{J} 04221332+1934$ & $\ldots$ & $\ldots$ & $\ldots$ & $\ldots$ & $\ldots$ & $\ldots$ & $\ldots$ & 4 \\
\hline $11-079$ & CFHT-Tau 21 & 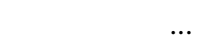 & $\ldots$ & 45 & $\mathrm{C}$ & 18 & $\ldots$ & $\ldots$ & 2 \\
\hline 02-013 & FV Tau AB & $-7.70 /-6.23$ & 58,44 & $6-23$ & $\mathrm{C} / \mathrm{C}$ & 58,7 & II & $23,1,27$ & 2 \\
\hline $02-000$ & FV Tau/c AB & $<-8.70 /<-8.60$ & 20,58 & $17-29$ & $\mathrm{C} / \mathrm{C}$ & 58,21 & II & $23,1,27$ & 2 \\
\hline $02-016$ & KPNO-Tau 13 & $\ldots$ & $\ldots$ & $8-10$ & W & 41,34 & $\ldots$ & $\ldots$ & 3 \\
\hline $02-000$ & DG Tau B & & $\ldots$ & $>276$ & $\mathrm{C}^{*}$ & 59 & I/II;II & $23 ; 59$ & 1 \\
\hline $02-022$ & DG Tau A & $-7.34 /-6.13$ & 58,59 & $63-125$ & $\mathrm{C}^{*}$ & 59,29 & I/II;II & $23 ; 59,27$ & 2 \\
\hline $02-000$ & KPNO-Tau 4 & $<-12.00$ & 46 & $38-158$ & $\mathrm{C}$ & 46,18 & III & 23 & 4 \\
\hline $02-000$ & IRAS $04248+2612 \mathrm{AB}$ & -8.97 & 59 & 163 & $\mathrm{C}^{*}$ & 59 & I & $59,1,27$ & 1 \\
\hline $15-020$ & JH 507 & & $\ldots$ & $\ldots$ & $\ldots$ & $\ldots$ & III & $23,1,27$ & 3 \\
\hline 13-004 & GV Tau AB & -6.71 & 59 & 86 & $\mathrm{C}^{*}$ & 59 & I & $59,1,27$ & 1 \\
\hline $13-000$ & IRAS $04264+2433$ & -7.11 & 59 & $69-96$ & $\mathrm{C}^{*}$ & 29,59 & I & 59,27 & 1 \\
\hline $15-040$ & DH Tau AB & $-8.95 /-8.30$ & 58,44 & $39-72$ & $\mathrm{C}$ & 29,58 & II & $23,1,27$ & 2 \\
\hline $15-042$ & DI Tau AB & & $\ldots$ & $1-2$ & W & 14,29 & III;II & $23 ; 27$ & 3 \\
\hline $15-044$ & KPNO-Tau 5 & $<-12.00$ & 46 & $4-30$ & W & 46,5 & III & 23 & 4 \\
\hline 14-006 & IQ Tau A & $<-8.32 /-7.55$ & 58,44 & $8-17$ & $\mathrm{C}$ & 29,21 & II & 27 & 2 \\
\hline $13-000$ & CFHT-Tau 20 & & $\ldots$ & 114 & $\mathrm{C}$ & 18 & $\ldots$ & $\ldots$ & 2 \\
\hline $14-000$ & KPNO-Tau 6 & -11.40 & 46 & $41-350$ & $\mathrm{C}$ & 46,5 & II & 23 & 4 \\
\hline $13-035$ & FX Tau AB & -8.65 & 44 & $10-15$ & $\mathrm{C} / \mathrm{W}$ & 7,29 & II & 1,27 & 2 \\
\hline $14-057$ & DK Tau AB & -7.42 & 44 & $31-50$ & $\mathrm{C} / \mathrm{C}$ & 26,29 & II & 1,27 & 2 \\
\hline $14-000$ & KPNO-Tau 7 & -11.40 & 46 & $31-300$ & $\mathrm{C}$ & 46,5 & II & 23 & 4 \\
\hline $22-013$ & MHO 9 & $<-9.70$ & 57 & 3-6 & W & 41,4 & $\ldots$ & $\ldots$ & 3 \\
\hline $22-021$ & MHO 4 & $<-9.50$ & 57 & $28-43$ & $\mathrm{~W}$ ? & 57,45 & $\ldots$ & $\ldots$ & 4 \\
\hline $22-040$ & L1551 IRS5 & $\ldots$ & $\ldots$ & $83-412$ & $\mathrm{C}^{*}$ & 29,59 & I & $59,1,27$ & 1 \\
\hline $22-042$ & LkHa 358 & -8.50 & 45 & $47-87$ & $\mathrm{C}$ & 7,29 & II & 27 & 2 \\
\hline $22-000$ & HH 30 & -6.45 & 59 & $185-199$ & $\mathrm{C}$ & 29,59 & II & 59 & 2 \\
\hline $22-043$ & HL Tau & $-8.83 /-6.80$ & 58,59 & $43-55$ & $\mathrm{C}^{*}$ & 59,7 & I;II & 1,$59 ; 27$ & 1 \\
\hline $22-047$ & XZ Tau AB & $-8.90 /-7.00$ & 58,20 & $62-274$ & $\mathrm{C} / \mathrm{C}$ & 58,7 & II & 59,27 & 2 \\
\hline $22-056$ & L1551 NE & $\ldots$ & $\ldots$ & $\ldots$ & $\ldots$ & $\ldots$ & I & $59,1,27$ & 1 \\
\hline 03-005 & HK Tau AB & -7.65 & 59 & $29-54$ & $\mathrm{C} / \mathrm{C}$ & 7,29 & $\mathrm{FS} ; \mathrm{I} / \mathrm{II}$ & $1 ; 27,59$ & 2 \\
\hline $22-070$ & V710 Tau BA & $\ldots$ & $\ldots$ & $34-89$ & $\mathrm{C} / \mathrm{W}$ & 37,26 & II + II & 1,27 & 2 \\
\hline 19-009 & JH 665 & $\ldots$ & $\ldots$ & $\ldots$ & $\ldots$ & $\ldots$ & III & 27 & 3 \\
\hline $22-089$ & L1551 51 & $<-9.50$ & 58 & $1-2$ & W & 37,29 & III & 1,27 & 3 \\
\hline $22-097$ & V827 Tau & $<-8.53$ & 58 & 2-4 & $\mathrm{W}$ & 7,29 & III & 1,27 & 3 \\
\hline 03-016 & Haro 6-13 & -7.54 & 59 & $34-88$ & $\mathrm{C}$ & 59,7 & FS;I/II & $1 ; 27,59$ & 2 \\
\hline
\end{tabular}


Table 11. continued.

\begin{tabular}{|c|c|c|c|c|c|c|c|c|c|}
\hline XEST & Name & $\begin{array}{r}\dot{M}(\min / \max )^{a} \\
\left(M_{\odot} \mathrm{yr}^{-1}\right)\end{array}$ & Refs. & $\begin{array}{r}E W(\mathrm{H} \alpha)^{b} \\
(\AA)\end{array}$ & $\begin{array}{l}\text { TTS } \\
\text { type }\end{array}$ & Refs. $^{c}$ & $\begin{array}{l}\mathrm{IR}^{d} \\
\text { class }\end{array}$ & Refs. $^{d}$ & Type \\
\hline $22-100$ & V826 Tau & & $\ldots$ & $2-4$ & $\mathrm{~W}$ & 7,29 & III & 1,27 & 3 \\
\hline $22-101$ & MHO 5 & $-10.80 /<-9.70$ & 45,57 & $36-60$ & C? & 57,45 & $\ldots$ & $\ldots$ & 2 \\
\hline 03-017 & CFHT-Tau 7 & $\ldots$ & $\ldots$ & 9 & W & 18 & $\ldots$ & $\ldots$ & 3 \\
\hline 03-019 & V928 Tau AB & $\ldots$ & $\ldots$ & $1-2$ & W & 37,29 & III & 1,27 & 3 \\
\hline 03-022 & FY Tau & -7.48 & 58 & $48-73$ & $\mathrm{C}$ & 58,29 & II & 1,27 & 2 \\
\hline $03-023$ & FZ Tau & -7.70 & 58 & $181-204$ & $\mathrm{C}$ & 29,7 & II & 1,27 & 2 \\
\hline $17-002$ & IRAS $04295+2251$ & $\ldots$ & $\ldots$ & $>11-66$ & $\mathrm{C}^{*}$ & 59,29 & $\mathrm{I} ; \mathrm{FS}$ & 59,$27 ; 1$ & 1 \\
\hline $19-049$ & UZ Tau E+W(AB) & $-8.70 /-8.01$ & 58,20 & $65-82$ & $\mathrm{C} / \mathrm{C} / \mathrm{C}$ & 29,7 & II+II & 23 & 2 \\
\hline 17-009 & JH 112 & $\ldots$ & $\ldots$ & 16 & $\mathrm{C}$ & 29 & II & 1,27 & 2 \\
\hline 03-031 & CFHT-Tau 5 & $\ldots$ & $\ldots$ & 30 & W & 18 & $\ldots$ & $\ldots$ & 4 \\
\hline 04-003 & CFHT-Tau 5 & $\ldots$ & $\ldots$ & 30 & W & 18 & $\ldots$ & $\ldots$ & 4 \\
\hline 03-035 & MHO 8 & $\ldots$ & $\ldots$ & $14-21$ & $\mathrm{~W}$ & 45,4 & II & 22 & 3 \\
\hline 04-009 & MHO 8 & $\ldots$ & $\ldots$ & $14-21$ & W & 45,4 & II & 22 & 3 \\
\hline 04-010 & GH Tau AB & $-8.90 /-7.92$ & 58,44 & $10-31$ & $\mathrm{C} / \mathrm{C}$ & 20,58 & II & 1,27 & 2 \\
\hline 04-012 & V807 Tau SNab & $<-8.68 /-8.40$ & 20,58 & $5-16$ & $\mathrm{C} / \mathrm{W}$ & 21,37 & III;II & $1 ; 27$ & 2 \\
\hline 18-004 & KPNO-Tau 14 & $<-12.00$ & 46 & $14-40$ & $\mathrm{~W} ?$ & 46,34 & $\ldots$ & $\ldots$ & 3 \\
\hline $17-000$ & CFHT-Tau 12 & & $\ldots$ & 80 & $\mathrm{C}$ & 18 & $\ldots$ & $\ldots$ & 2 \\
\hline 04-016 & V830 Tau & $<-8.97$ & 58 & $2-3$ & $\mathrm{~W}$ & 29,60 & III & 1,27 & 3 \\
\hline $18-000$ & IRAS S04301+261 & $\ldots$ & $\ldots$ & $\ldots$ & $\ldots$ & $\ldots$ & II & 23,27 & 2 \\
\hline $17-000$ & IRAS $04302+2247$ & $\ldots$ & $\ldots$ & 67 & $\mathrm{C}^{*}$ & 59 & I & $59,1,27$ & 1 \\
\hline $17-027$ & IRAS $04303+2240$ & $-6.63 /-6.05$ & 59 & $67-137$ & $\mathrm{C}$ & 59 & II & 59,27 & 2 \\
\hline 04-034 & GI Tau & -8.08 & 58,44 & $15-21$ & $\mathrm{C}$ & 29,21 & II & 27 & 2 \\
\hline 04-035 & GK Tau AB & -8.19 & 58 & $15-35$ & $\mathrm{C} / \mathrm{C}$ & 21,11 & II & $59,1,27$ & 2 \\
\hline 18-019 & IS Tau AB & $-8.10 /-7.91$ & 58,20 & $10-26$ & $\mathrm{C} / \mathrm{C}$ & 20,58 & II & $1,23,27$ & 2 \\
\hline $17-058$ & CI Tau & $-7.59 /-7.19$ & 58,44 & $77-102$ & $\mathrm{C}$ & 29,7 & II & 1,27 & 2 \\
\hline $18-030$ & IT Tau AB & $\ldots$ & $\ldots$ & $10-22$ & $\mathrm{C} / \mathrm{C}$ & 29,11 & II & $1,23,27$ & 2 \\
\hline $17-066$ & JH 108 & $\ldots$ & $\ldots$ & 3 & $\mathrm{~W}$ & 29 & III & 1,27 & 3 \\
\hline $17-068$ & CFHT-BD Tau 1 & $\ldots$ & $\ldots$ & $7-19$ & W & 41,39 & $\ldots$ & $\ldots$ & 4 \\
\hline $25-026$ & AA Tau & $-8.48 /-8.19$ & 44,58 & $37-46$ & $\mathrm{C}$ & 7,29 & II & 1,27 & 2 \\
\hline 09-010 & HO Tau AB & -8.87 & 58 & $102-115$ & $\mathrm{C}$ & 29,7 & II & 1,27 & 2 \\
\hline 08-019 & FF Tau AB & & $\ldots$ & $1-3$ & W & 24,29 & III & 1,27 & 3 \\
\hline $12-040$ & DN Tau & $-8.73 /-7.79$ & 58,59 & $12-87$ & $\mathrm{C}$ & 7,14 & II & $59,1,27$ & 2 \\
\hline $12-000$ & IRAS $04325+2402 \mathrm{AB}$ & $\ldots$ & $\ldots$ & $\ldots$ & $\ldots$ & $\ldots$ & I & $59,1,27$ & 1 \\
\hline $12-059$ & CoKu Tau 3 AB & $\ldots$ & $\ldots$ & 5 & W & 7 & II & 1,27 & 3 \\
\hline 09-022 & KPNO-Tau 8 & $\ldots$ & $\ldots$ & $15-18$ & $\mathrm{~W}$ & 41,5 & $\ldots$ & $\ldots$ & 3 \\
\hline 08-037 & HQ Tau AB & $\ldots$ & $\ldots$ & $\ldots$ & $\ldots$ & $\ldots$ & III & 1 & 3 \\
\hline 09-026 & HQ Tau AB & $\ldots$ & $\ldots$ & $\ldots$ & $\ldots$ & $\ldots$ & III & 1 & 3 \\
\hline 08-043 & KPNO-Tau 15 & $\ldots$ & $\ldots$ & 6 & $\mathrm{~W}$ & 34 & $\ldots$ & $\ldots$ & 3 \\
\hline 09-031 & KPNO-Tau 15 & $\ldots$ & $\ldots$ & 6 & W & 34 & $\ldots$ & $\ldots$ & 3 \\
\hline 08-000 & KPNO-Tau 9 & $\ldots$ & $\ldots$ & 1 & W & 41 & $\ldots$ & $\ldots$ & 4 \\
\hline 09-000 & KPNO-Tau 9 & $\ldots$ & $\ldots$ & 1 & $\mathrm{~W}$ & 41 & $\ldots$ & $\ldots$ & 4 \\
\hline 08-048 & HP Tau AB & $\ldots$ & $\ldots$ & $20-35$ & $\mathrm{C}$ & 29,7 & FS;II & $1 ; 27$ & 2 \\
\hline 08-051a & HP Tau/G3 AB & $\ldots$ & $\ldots$ & $1-2$ & $\mathrm{~W}$ & 21,29 & III & 27 & 3 \\
\hline 08-051 & HP Tau/G2 & $\ldots$ & $\ldots$ & $0-5$ & W & 21,7 & III & 27 & 3 \\
\hline $08-058$ & Haro 6-28 AB & $-8.70 /-8.10$ & 45,20 & $48-92$ & $\mathrm{C} / \mathrm{C}$ & 45,7 & $\mathrm{FS} ; \mathrm{I}$ & $1 ; 59,27$ & 2 \\
\hline 08-000 & CFHT-BD Tau 2 & $\ldots$ & $\ldots$ & $7-13$ & $\mathrm{~W}$ & 41,39 & $\ldots$ & $\ldots$ & 4 \\
\hline 08-080 & CFHT-BD Tau 3 & $<-12.00$ & 46 & $11-55$ & $\mathrm{~W}$ ? & 46,39 & $\ldots$ & $\ldots$ & 4 \\
\hline 05-005 & CFHT-Tau 6 & -11.30 & 46 & 64-102 & $\mathrm{C}$ & 18,46 & $\ldots$ & $\ldots$ & 4 \\
\hline $05-000$ & IRAS $04361+2547$ & $\ldots$ & $\ldots$ & $\ldots$ & $\ldots$ & $\ldots$ & I & $59,1,27$ & 1 \\
\hline 05-013 & GN Tau AB & -7.90 & 57 & $59-62$ & $\mathrm{C}$ & 57,3 & II & $1,23,27$ & 2 \\
\hline 05-017 & IRAS $04365+2535$ & $\ldots$ & $\ldots$ & $\ldots$ & $\ldots$ & $\ldots$ & I & $1,23,59,27$ & 1 \\
\hline $05-024$ & IRAS $04369+2539$ & -6.20 & 59 & 22 & $\mathrm{C}$ & 59 & II & $1,59,27$ & 2 \\
\hline 07-011 & JH 223 & $\ldots$ & $\ldots$ & 4 & $\mathrm{~W}$ & 29 & II & $1,23,27$ & 3 \\
\hline 07-022 & Haro 6-32 & $\ldots$ & $\ldots$ & $\ldots$ & $\mathrm{W}$ & 33 & $\ldots$ & $\ldots$ & 3 \\
\hline 07-000 & ITG $33 \mathrm{~A}$ & $\ldots$ & $\ldots$ & 53 & $\mathrm{C}$ & 35 & $\ldots$ & $\ldots$ & 2 \\
\hline $07-000$ & CFHT-Tau 8 & $\cdots$ & $\begin{array}{l}\cdots \\
\cdots\end{array}$ & 52 & $\mathrm{C}$ & 18,33 & $\cdots$ & $\begin{array}{l}\cdots \\
\cdots\end{array}$ & 2 \\
\hline 07-000 & IRAS $04381+2540$ & $\ldots$ & $\ldots$ & $\ldots$ & $\ldots$ & $\ldots$ & I & $23,1,59,27$ & 1 \\
\hline $07-041$ & IRAS $04385+2550 \mathrm{AB}$ & -8.11 & 59 & $15-20$ & $\mathrm{C}$ & 59,29 & II;I & 23,$27 ; 59$ & 2 \\
\hline 10-017 & CoKuLk332/G2 AB & $<-8.58$ & 20 & $2-3$ & $\mathrm{~W} / \mathrm{W}$ & 20,7 & III & $1,23,27$ & 3 \\
\hline
\end{tabular}


Table 11. continued.

\begin{tabular}{|c|c|c|c|c|c|c|c|c|c|}
\hline XEST & Name & $\begin{array}{r}\dot{M}(\min / \max )^{a} \\
\left(M_{\odot} \mathrm{yr}^{-1}\right)\end{array}$ & Refs. & $\begin{array}{r}E W(\mathrm{H} \alpha)^{b} \\
(\AA)\end{array}$ & $\begin{array}{l}\text { TTS } \\
\text { type }\end{array}$ & Refs. $^{c}$ & $\begin{array}{r}\mathrm{IR}^{d} \\
\text { class }\end{array}$ & Refs. $^{d}$ & Type \\
\hline $10-018$ & CoKuLk332/G1 AB & $<8.20$ & 58 & $0-5$ & $\mathrm{~W} / \mathrm{W}$ & 58,29 & III;II & 23,$1 ; 27$ & 3 \\
\hline $10-020$ & V955 Tau AB & $<-8.97 /-8.50$ & 20,58 & $11-45$ & $\mathrm{C} / \mathrm{W}$ & 20,58 & II & $23,1,27$ & 2 \\
\hline $10-034$ & CIDA 7 & & $\ldots$ & 79 & $\mathrm{C}$ & 3 & II & 23,1 & 2 \\
\hline $10-045$ & DP Tau & $-8.50 /-6.92$ & 58,59 & $74-102$ & $\mathrm{C}$ & 59,29 & II & $23,1,59,27$ & 2 \\
\hline $10-060$ & GO Tau & -8.42 & 58 & $78-81$ & $\mathrm{C}$ & 29,7 & II & $23,1,27$ & 2 \\
\hline $26-012$ & $2 \mathrm{M} \mathrm{J} 04552333+30$ & $\ldots$ & $\ldots$ & $\ldots$ & $\mathrm{W}$ & 33 & $\ldots$ & $\ldots$ & 4 \\
\hline $26-034$ & $2 \mathrm{M} \mathrm{J} 04554046+30$ & $\ldots$ & $\ldots$ & $\ldots$ & W & 33 & $\ldots$ & $\ldots$ & 3 \\
\hline $26-043$ & AB Aur & $\ldots$ & $\ldots$ & $22-44$ & $\mathrm{Ae}$ & 15,29 & II & 1,27 & 5 \\
\hline $26-050$ & 2MJ04554757/801 & $\ldots$ & $\ldots$ & 25 & $\mathrm{C}$ & 46 & $\ldots$ & $\ldots$ & 2 \\
\hline $26-067$ & SU Aur & $-8.30 /-8.20$ & 63 & $2-6$ & $\mathrm{C}$ & 29,14 & II & 1,27 & 2 \\
\hline $26-072$ & HBC 427 & $\ldots$ & $\ldots$ & 1 & W & 29 & III & $23,1,27$ & 3 \\
\hline \multicolumn{10}{|c|}{ Additional sources from Chandra } \\
\hline$\overline{\mathrm{C} 1-0}$ & KPNO-Tau 10 & $\ldots$ & $\ldots$ & 36 & $\bar{C}$ & 34 & & 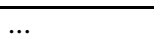 & 2 \\
\hline C1-1 & IRAS $04158+2805$ & $<-9.50$ & 59 & $\ldots$ & $\ldots$ & $\ldots$ & $\mathrm{I} ; \mathrm{II}$ & $59 ; 27$ & 2 \\
\hline C2-1 & Haro 6-5 B & -6.76 & 59 & 91 & $\mathrm{C}^{*}$ & 59 & II & 59 & 1 \\
\hline C2-2 & FS Tau AC & $-9.50 /-8.28$ & 58,20 & $57-81$ & $\mathrm{C} / \mathrm{C}$ & 7,58 & FS;II & $1 ; 27$ & 2 \\
\hline C3-1 & FV Tau/c AB & $<-8.70 /<-8.60$ & 20,58 & $17-21$ & $\mathrm{C} / \mathrm{C}$ & 58,20 & II & $23,1,27$ & 2 \\
\hline C3-2 & DG Tau B & $\ldots$ & $\ldots$ & $>276$ & $C^{*}$ & 59 & I/II;II & $23 ; 59$ & 1 \\
\hline C4-1 & GV Tau AB & -6.71 & 59 & 86 & $\mathrm{C}^{*}$ & 59 & I & $59,1,27$ & 1 \\
\hline C5-2 & HN Tau AB & $\ldots$ & $\ldots$ & $138-163$ & $\mathrm{C}$ & 21,58 & II & 1,27 & 2 \\
\hline C5-1 & L1551 55 & $<-9.70$ & 58 & 1 & W & 58 & III & 1,27 & 3 \\
\hline C5-4 & HD 28867 & & $\ldots$ & $\ldots$ & $\ldots$ & $\ldots$ & 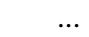 & & 9 \\
\hline C5-3 & DM Tau & -8.67 & 58 & $\ldots$ & $\ldots$ & $\ldots$ & II & 1,27 & 2 \\
\hline C6-1 & CFHT-BD Tau 4 & -11.30 & 46 & $129-340$ & $\mathrm{C}$ & 46,39 & $\ldots$ & $\ldots$ & 4 \\
\hline C6-0 & L1527 IRS & $\ldots$ & $\ldots$ & $\ldots$ & $\ldots$ & $\ldots$ & 0 & 59,23 & 0 \\
\hline C6-0 & CFHT-Tau 17 & $\ldots$ & $\ldots$ & 7 & W & 18 & $\ldots$ & $\ldots$ & 3 \\
\hline C6-2 & IRAS $04370+2559$ & $\ldots$ & $\ldots$ & $\ldots$ & $\ldots$ & $\ldots$ & II & 27 & 2 \\
\hline
\end{tabular}

${ }^{a}$ Range of $\dot{M}$ reported in literature given. For multiple systems, numbers refer to primary or integrated system.

${ }^{b}$ Range of $E W$ reported in literature given. For multiple systems, numbers refer to primary or integrated system.

${ }^{c}$ For $E W$ range, first reference for minimum, second for maximum reported.

${ }^{d}$ Infrared classification; double entries: '/' for transition objects, '+' for components, ';' for different types, ',' for different references. FS = flat-spectrum source. 


\section{Appendix A: The XEST source catalog}

The complete catalog of all X-ray sources detected in the XEST fields (not including the complementary fields from Chandra) is available in electronic form. The data reduction and source identification procedures are described in Sect. 4. The catalog also provides errors of the source counts and the count rates (not given in Table 4 for the "Scts" and "Rate" columns). The numbering scheme is as defined in Sect. 5, all TMC members being included. The catalog is sorted in right ascension and contains a total of 2347 identified X-ray sources. If two XEST fields overlap, the same sources may have been identified twice, with different XEST IDs assigned. The first ten entries of the catalog are given in the Table A.1 below for illustration. Note that if $\mathrm{ML}<5$ in any band the number of counts and the count rate are $95 \%$ upper limits in that band. The HR is the upper (or lower) limit as appropriate in these situations with the low and high values set to -1 and to the HR upper limit, respectively (or to the HR lower limit and to +1 , respectively).

A description of the columns follows:

1 XEST ID (field-srenr).

2 RA (J2000, X-ray, corrected for boresight shift).

3 Dec (J2000, X-ray, corrected for boresight shift).

4 RA as above, in decimal degrees.

5 Dec as above, in decimal degrees.

$61 \sigma$ positional error (including rms of boresight shift).

7 Maximum likelihood of detection in the mosaicked EPIC full band (0.5-7.3 keV) image.

8 Number of detected counts in the mosaicked EPIC full band image. If $\mathrm{ML}_{\mathrm{F}}<5$, then $95 \%$ upper limit is given.

9 Total PN-equivalent effective exposure time in $\mathrm{s}$ at source position in in the mosaicked EPIC full band image.
10 PN-equivalent source count-rate in the mosaicked EPIC full band image. If $\mathrm{ML}_{\mathrm{F}}<5$, then $95 \%$ upper limit is given.

$111 \sigma$ statistical error in PN-equivalent source count-rate in the mosaicked EPIC full band image (note: does not include uncertainty in spectrally-dependent PN/MOS sensitivity ratio).

12 Maximum likelihood of detection in the mosaicked EPIC soft band $(0.5-2 \mathrm{keV})$ image.

13 Number of detected counts in the mosaicked EPIC soft band image. If $\mathrm{ML}_{\mathrm{S}}<5$, then $95 \%$ upper limit is given.

14 Total PN-equivalent effective exposure time in $\mathrm{s}$ at source position in in the mosaicked EPIC soft band image.

15 PN-equivalent source count-rate in the mosaicked EPIC soft band image. If $\mathrm{ML}_{\mathrm{S}}<5$, then $95 \%$ upper limit is given.

$161 \sigma$ statistical error in PN-equivalent source count-rate in the mosaicked EPIC soft band image (note: does not include uncertainty in spectrally-dependent $\mathrm{PN} / \mathrm{MOS}$ sensitivity ratio).

17 Maximum likelihood of detection in the mosaicked EPIC hard band (2-7.3 keV) image.

18 Number of detected counts in the mosaicked EPIC hard band image. If $\mathrm{ML}_{\mathrm{H}}<5$, then $95 \%$ upper limit is given.

19 Total PN-equivalent effective exposure time in $\mathrm{s}$ at source position in in the mosaicked EPIC hard band image.

20 PN-equivalent source count-rate in the mosaicked EPIC hard band image. If $\mathrm{ML}_{\mathrm{H}}<5$, then $95 \%$ upper limit is given.

$211 \sigma$ statistical error in PN-equivalent source count-rate in the mosaicked EPIC hard band image (note: does not include uncertainty in spectrally-dependent PN/MOS sensitivity ratio).

22 Hardness ratio $\left(\right.$ Rate $_{\mathrm{H}}-$ Rate $_{\mathrm{S}}$ )/Rate $\mathrm{R}_{\mathrm{F}}$. If object is not detected in soft (hard) band, then HR is upper (lower) limit. If object is detected only in full band, then $\mathrm{HR}=0$.

$231 \sigma$ lower limit to hardness ratio.

$241 \sigma$ upper limit to hardness ratio.

25 Comment concerning by-eye verification of source.

Table A.1. XEST Catalog (first ten entries).

\begin{tabular}{|c|c|c|c|c|c|c|c|c|c|c|}
\hline $\begin{array}{r}\text { XEST } \\
1\end{array}$ & $\begin{array}{r}\mathrm{RA}_{\mathrm{X}} \\
2\end{array}$ & $\begin{array}{r}\operatorname{Dec}_{X} \\
3\end{array}$ & $\begin{array}{r}\text { RA } \\
4\end{array}$ & $\begin{array}{r}\text { Dec } \\
5\end{array}$ & $\begin{array}{r}\text { Poserr } \\
6\end{array}$ & $\begin{array}{r}\mathrm{ML}_{\mathrm{F}} \\
7\end{array}$ & $\begin{array}{r}N_{\mathrm{F}} \\
8\end{array}$ & $\begin{array}{r}T_{\text {exp,F }} \\
9\end{array}$ & $\begin{array}{r}\text { Rate }_{F} \\
10\end{array}$ & $\begin{array}{r}\text { Err }_{F} \\
11\end{array}$ \\
\hline $27-001$ & 035303.92 & +315302.8 & 58.266348 & +31.884122 & 1.17 & 337.9 & 250 & 10386 & 0.0241 & 0.0017 \\
\hline $27-002$ & 5305.45 & 15419.8 & 58.272721 & +31.905495 & 1.47 & 20.0 & 41 & 11432 & 0.0037 & 0.0008 \\
\hline $27-003$ & 312.71 & 5512.7 & 02955 & 920193 & 1.28 & 51.7 & 102 & 27319 & 0.0038 & 0.0005 \\
\hline 27-004 & 315.59 & 5301.3 & 314976 & 83708 & 1.20 & 138.4 & 180 & 29916 & 0.0060 & 0.0006 \\
\hline $27-005$ & 319.30 & 4739.1 & 30416 & 794186 & 2.01 & 11.1 & 42 & 28822 & 0.0015 & 0.0004 \\
\hline $27-006$ & 19.73 & 21.4 & 216 & 89273 & 1.95 & 9.2 & 38 & 25935 & 0.0015 & 0.0004 \\
\hline $27-007$ & 035322.62 & +315624.9 & 58.344257 & +31.940258 & 1.66 & 11.1 & 38 & 31200 & 0.0012 & 0.0003 \\
\hline $27-008$ & 035324.35 & +314320.5 & 58.351468 & +31.722347 & 1.83 & 8.0 & 24 & 10810 & 0.0022 & 0.0007 \\
\hline $27-009$ & 5325.01 & +31 4213.4 & 58.354220 & +31.703724 & 1.60 & 8.8 & 24 & 9042 & 0.0027 & 0.0008 \\
\hline $27-010$ & 035325.25 & +320326.5 & 58.355189 & +32.057353 & 1.21 & 80.4 & 129 & 10663 & 0.0121 & 0.0014 \\
\hline
\end{tabular}

\begin{tabular}{rrrrrrrrrrrrr}
\hline \hline ML $_{\mathrm{S}}$ & $N_{\mathrm{S}}$ & $T_{\text {exp,S }}$ & Rate $_{\mathrm{S}}$ & Err $_{\mathrm{S}}$ & $\mathrm{ML}_{\mathrm{H}}$ & $\mathrm{N}_{\mathrm{H}}$ & $\mathrm{T}_{\text {exp, } \mathrm{H}}$ & Rate $_{\mathrm{H}}$ & Err $_{\mathrm{H}}$ & HR & HR $_{\text {low }}$ & HR $_{\text {high }}$ Verif. \\
12 & 13 & 14 & 15 & 16 & 17 & 18 & 19 & 20 & 21 & 22 & 23 & 24 \\
\hline 75.5 & 69 & 10471 & 0.0066 & 0.0009 & 271.6 & 181 & 10440 & 0.0174 & 0.0015 & +0.448 & +0.370 & +0.526 \\
17.8 & 29 & 11517 & 0.0026 & 0.0006 & 3.0 & 21 & 11503 & 0.0019 & 0.0000 & -0.160 & -1.000 & -0.160 \\
49.9 & 73 & 28589 & 0.0026 & 0.0004 & 9.1 & 32 & 27099 & 0.0012 & 0.0003 & -0.372 & -0.519 & -0.224 \\
106.7 & 115 & 31188 & 0.0037 & 0.0004 & 34.4 & 62 & 29754 & 0.0021 & 0.0004 & -0.265 & -0.361 & -0.169 \\
4.1 & 41 & 30079 & 0.0014 & 0.0000 & 6.4 & 24 & 28636 & 0.0009 & 0.0003 & -0.226 & -0.226 & +1.000 \\
8.4 & 27 & 27223 & 0.0010 & 0.0003 & 1.7 & 34 & 25697 & 0.0013 & 0.0000 & +0.142 & -1.000 & +0.142 \\
3.5 & 34 & 32427 & 0.0011 & 0.0000 & 5.8 & 22 & 31072 & 0.0007 & 0.0003 & -0.178 & -0.178 & +1.000 \\
0.2 & 11 & 10896 & 0.0011 & 0.0000 & 10.5 & 21 & 10894 & 0.0020 & 0.0005 & +0.297 & +0.297 & +1.000 \\
11.7 & 19 & 9131 & 0.0021 & 0.0006 & 0.3 & 19 & 9101 & 0.0022 & 0.0000 & +0.006 & -1.000 & +0.006 \\
112.0 & 132 & 11298 & 0.0117 & 0.0012 & 0.1 & 11 & 10463 & 0.0011 & 0.0000 & -0.825 & -1.000 & -0.825 \\
\hline
\end{tabular}




\section{Appendix B: The XEST atlas}

Figures B.1-B.10 show all 28 XEST EPIC exposures. Each field of view is presented in two versions. The left panel shows a coadded EPIC image, logarithmically compressed in intensity and slightly smoothed. The colors code for hardness, where hardness increases from red to yellow to green to blue. Saturated (bright) stellar images are black. The right panel shows an unsmoothed, co-added EPIC image with an RA(J2000.0) - Dec(J2000.0) coordinate grid. Also plotted are the locations of known TMC members (red circles), labeled with their XEST source IDs (srcnr). The latter are located directly above the source circles except in crowded areas where labels may have been shifted. 
M. Güdel et al.: XMM-Newton extended survey of Taurus, Online Material p 38
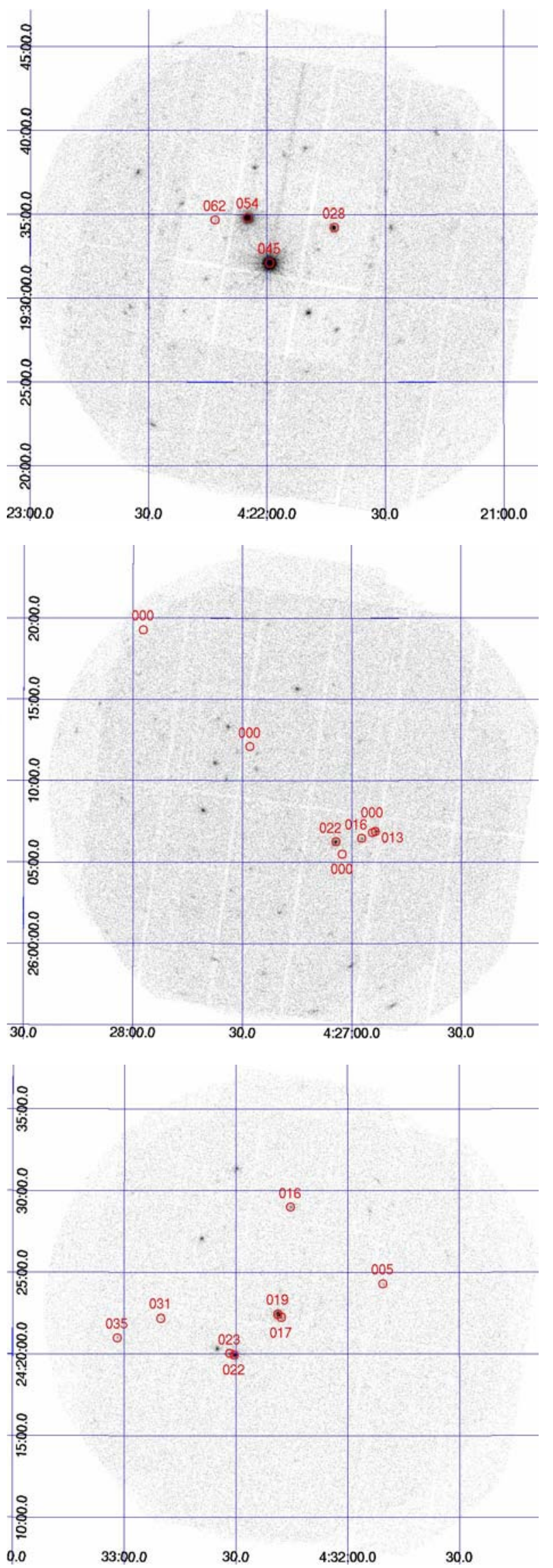

Fig. B.1. Co-added EPIC images of field XEST-01, XEST-02, and XEST-03 (from top to bottom). Left: Smoothed images, color coded for hardness; right: coordinate grid and TMC identifications included. 
M. Güdel et al.: XMM-Newton extended survey of Taurus, Online Material p 39

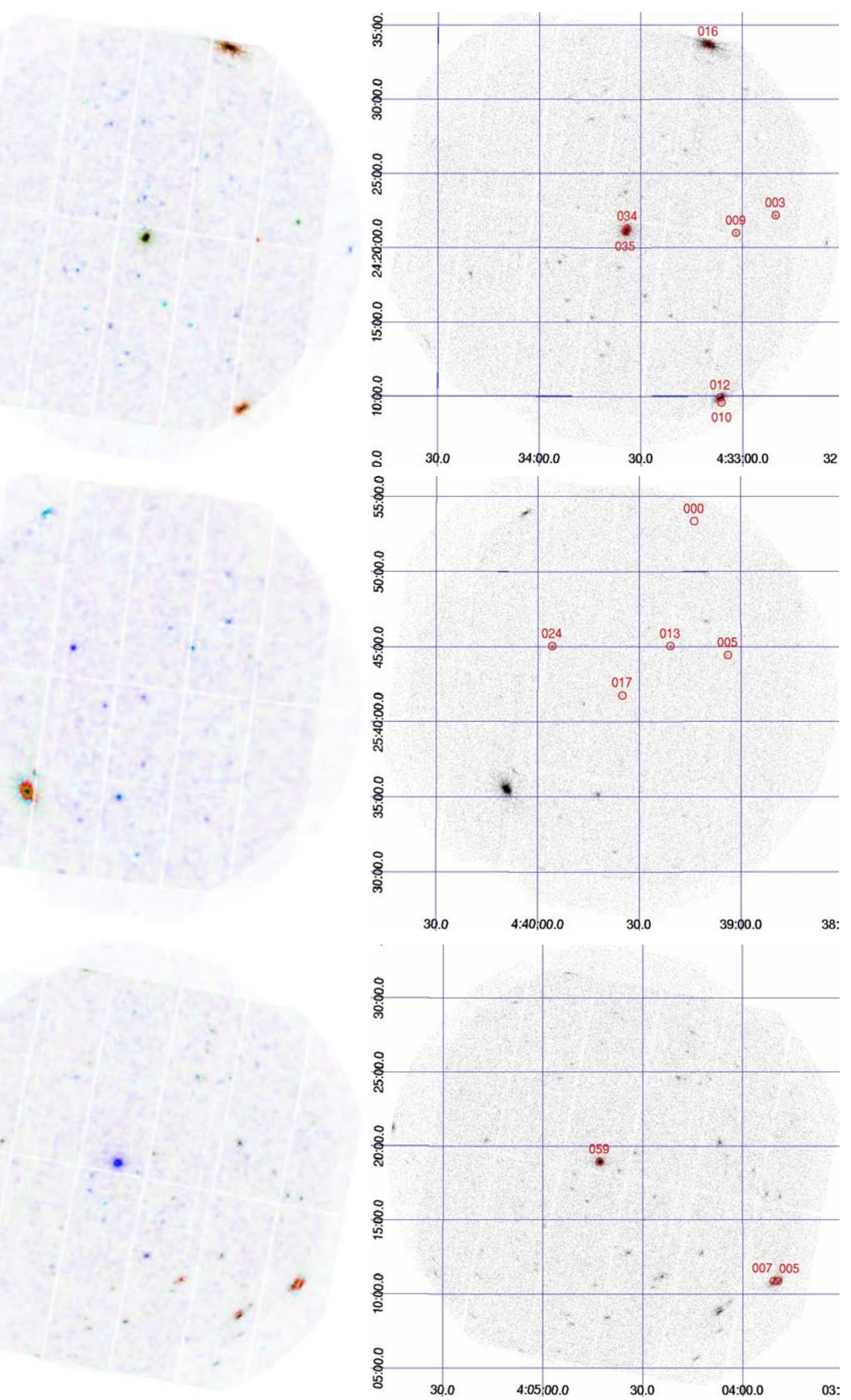

Fig. B.2. Co-added EPIC images of field XEST-04, XEST-05, and XEST-06 (from top to bottom). Left: Smoothed images, color coded for hardness; right: coordinate grid and TMC identifications included. 
M. Güdel et al.: XMM-Newton extended survey of Taurus, Online Material p 40
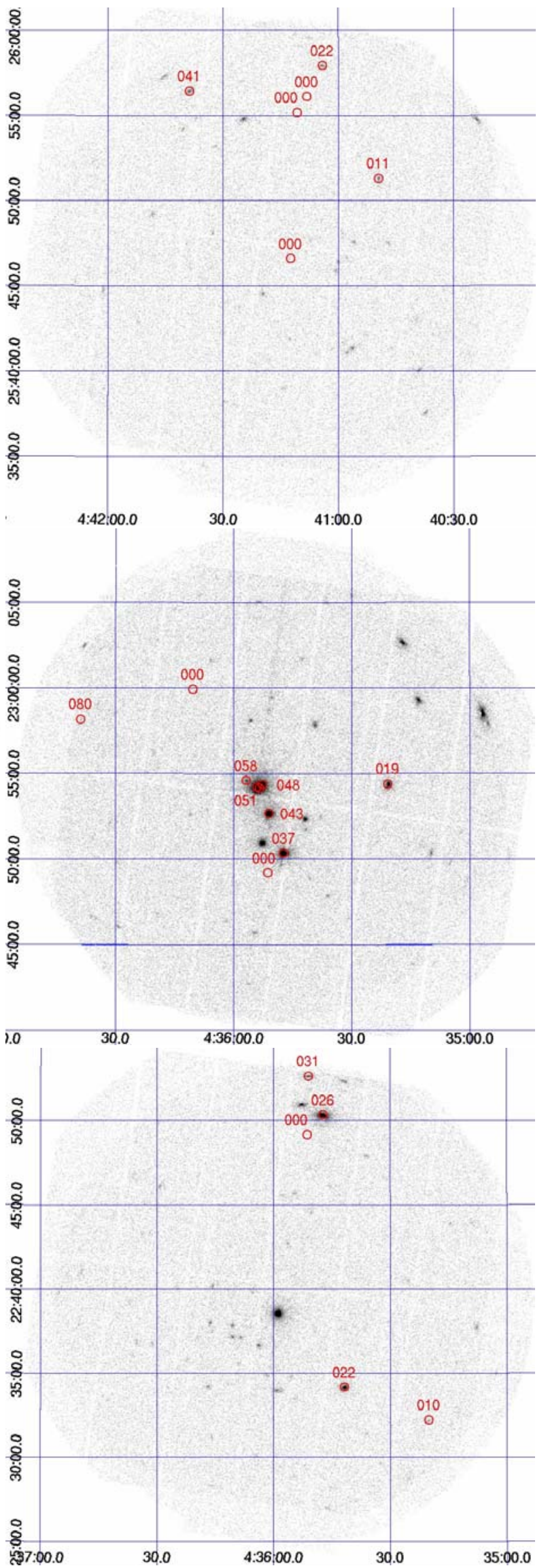

Fig. B.3. Co-added EPIC images of field XEST-07, XEST-08, and XEST-09 (from top to bottom). Left: Smoothed images, color coded for hardness; right: coordinate grid and TMC identifications included. 
M. Güdel et al.: XMM-Newton extended survey of Taurus, Online Material $p 41$

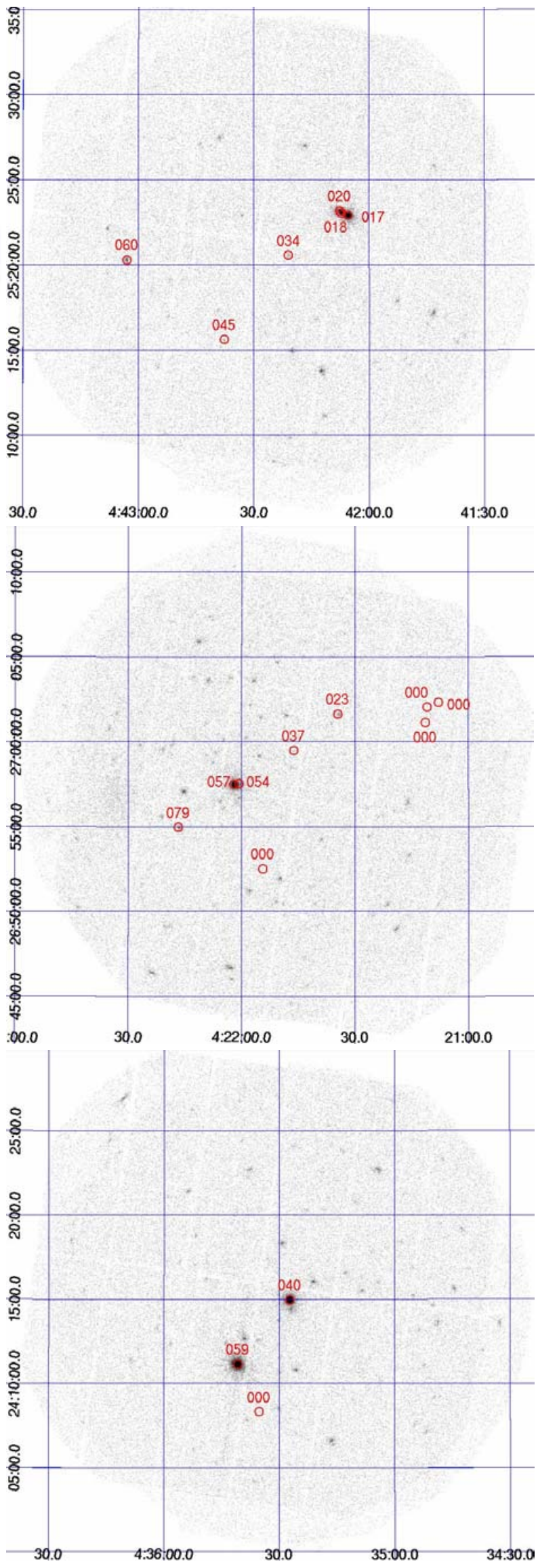

Fig. B.4. Co-added EPIC images of field XEST-10, XEST-11, and XEST-12 (from top to bottom). Left: Smoothed images, color coded for hardness; right: coordinate grid and TMC identifications included. 
M. Güdel et al.: XMM-Newton extended survey of Taurus, Online Material p 42

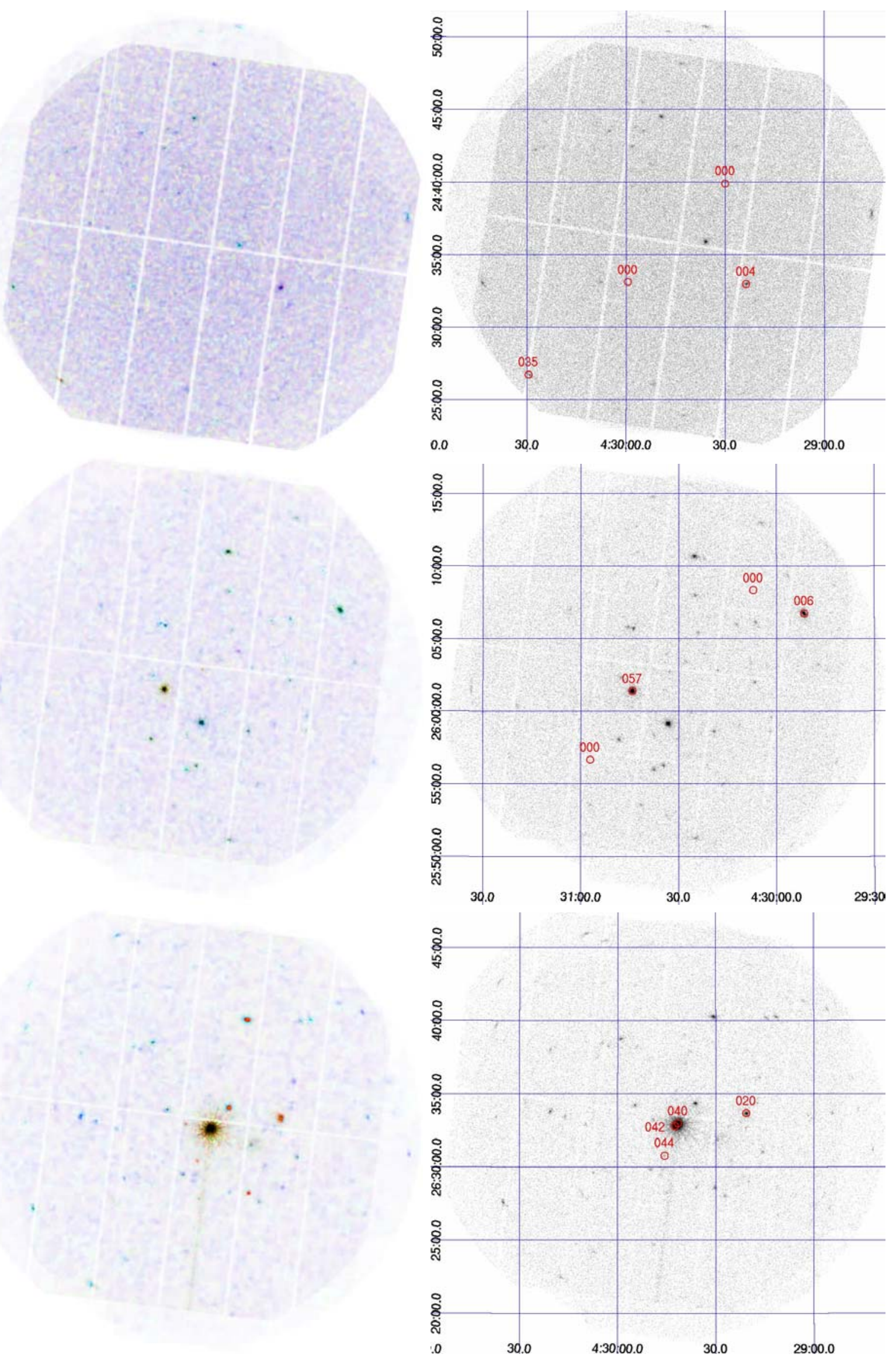

Fig. B.5. Co-added EPIC images of field XEST-13, XEST-14, and XEST-15 (from top to bottom). Left: Smoothed images, color coded for hardness; right: coordinate grid and TMC identifications included. 
M. Güdel et al.: XMM-Newton extended survey of Taurus, Online Material p 43
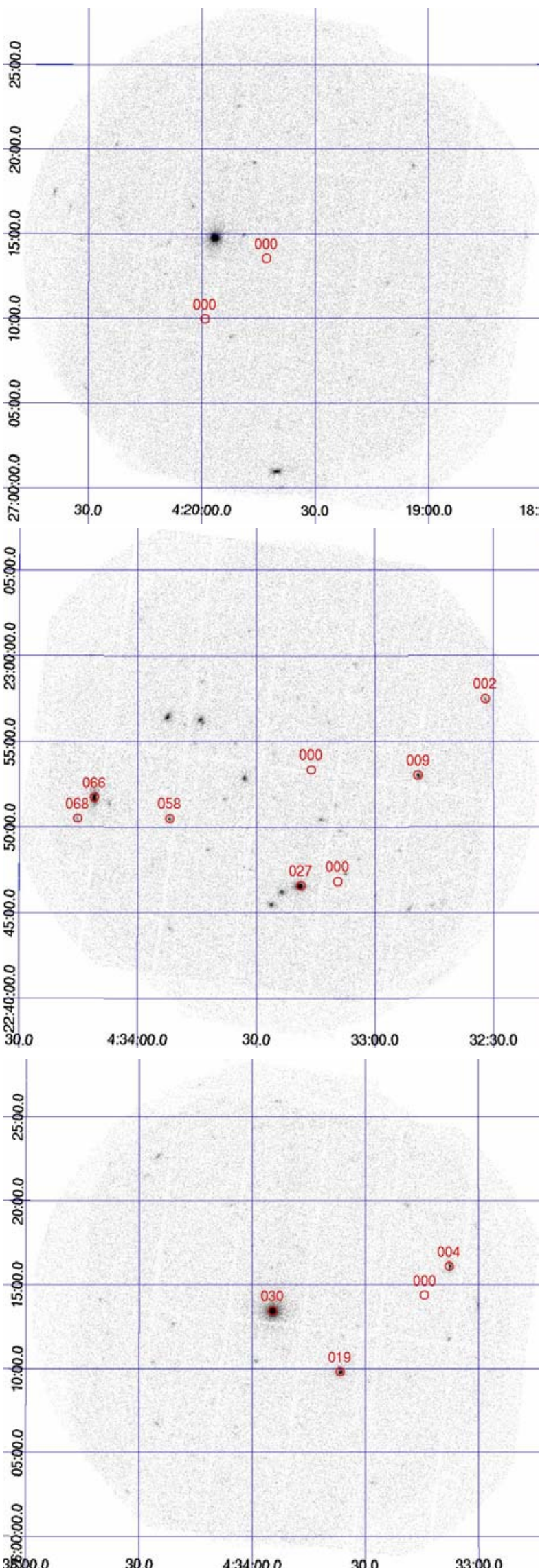

Fig. B.6. Co-added EPIC images of field XEST-16, XEST-17, and XEST-18 (from top to bottom). Left: Smoothed images, color coded for hardness; right: coordinate grid and TMC identifications included. 
M. Güdel et al.: XMM-Newton extended survey of Taurus, Online Material p 44

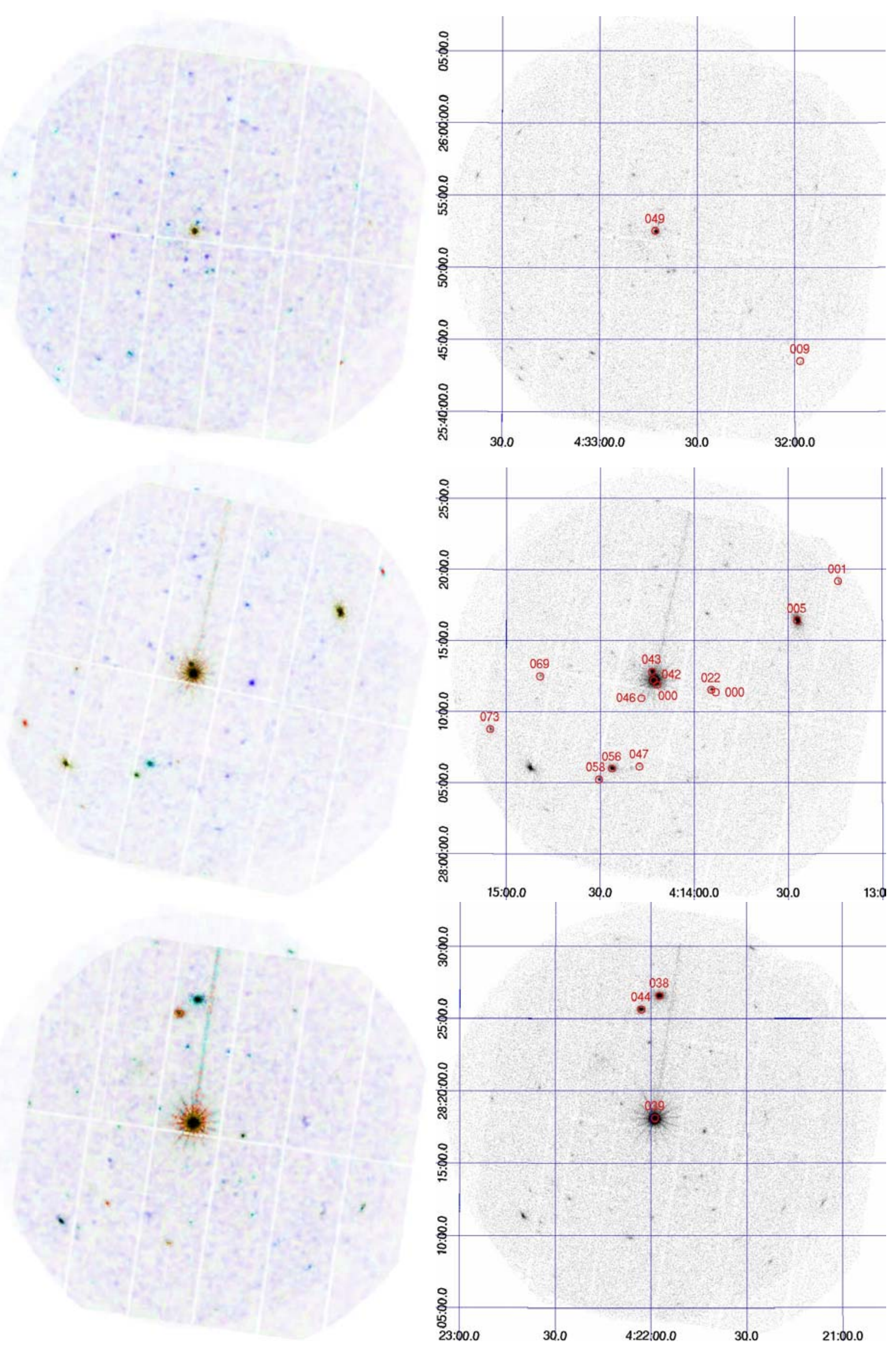

Fig. B.7. Co-added EPIC images of field XEST-19, XEST-20, and XEST-21 (from top to bottom). Left: Smoothed images, color coded for hardness; right: coordinate grid and TMC identifications included. 
M. Güdel et al.: XMM-Newton extended survey of Taurus, Online Material $p 45$

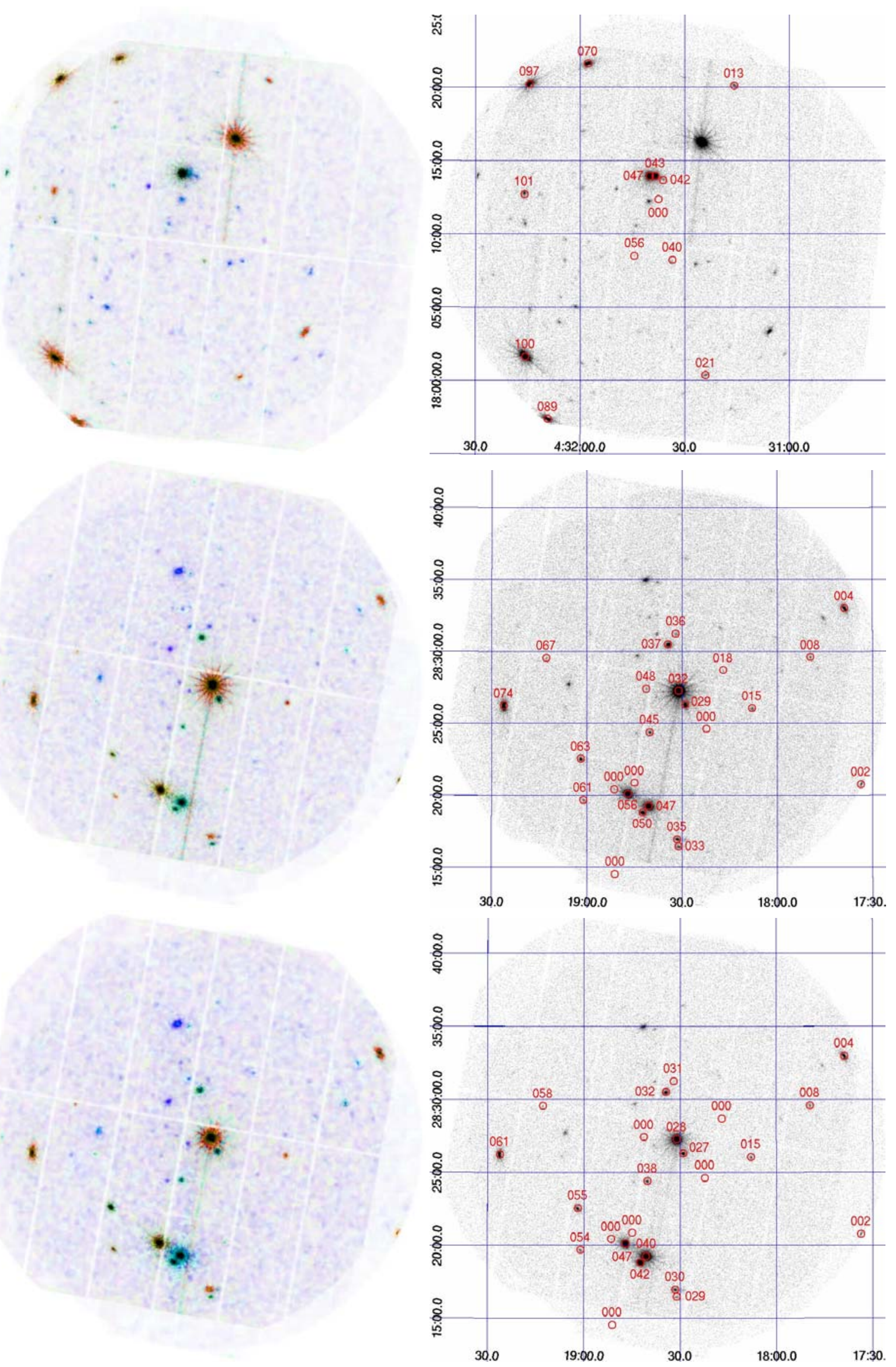

Fig. B.8. Co-added EPIC images of field XEST-22, XEST-23, and XEST-24 (from top to bottom). Left: Smoothed images, color coded for hardness; right: coordinate grid and TMC identifications included. 
M. Güdel et al.: XMM-Newton extended survey of Taurus, Online Material p 46
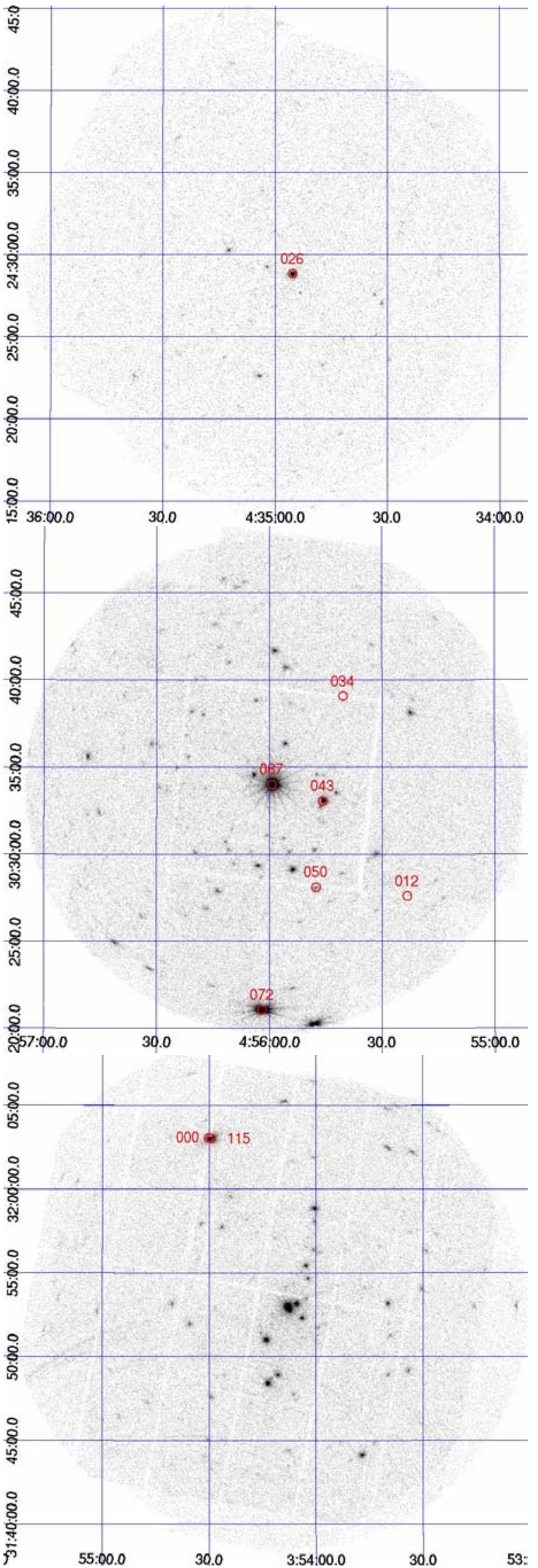

Fig. B.9. Co-added EPIC images of field XEST-25, XEST-26, and XEST-27 (from top to bottom). Left: Smoothed images, color coded for hardness; right: coordinate grid and TMC identifications included. 
M. Güdel et al.: XMM-Newton extended survey of Taurus, Online Material p 47
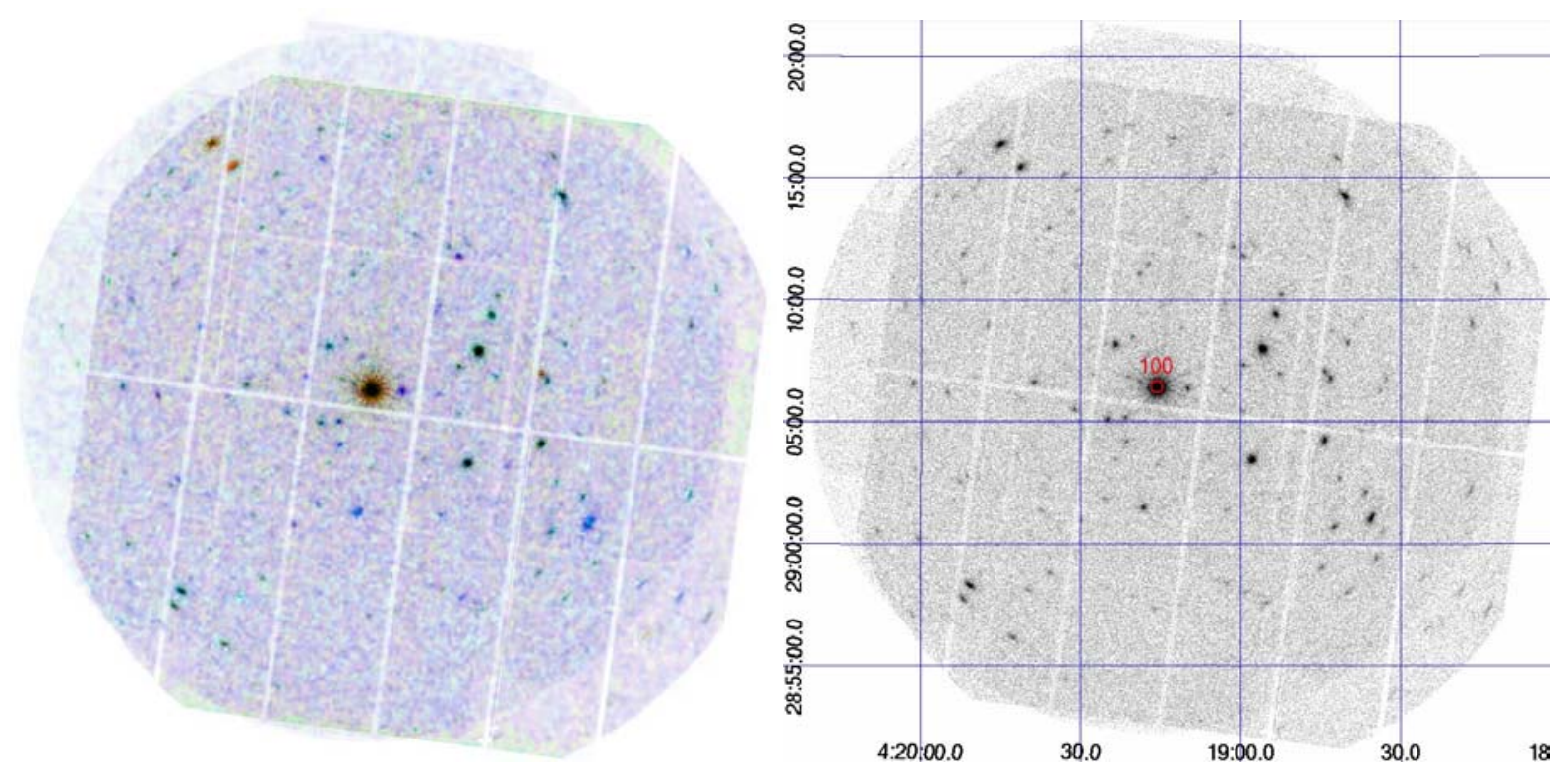

Fig. B.10. Co-added EPIC images of field XEST-28. Left: Smoothed image, color coded for hardness; right: coordinate grid and TMC identifications included. 